This is the post-peer reviewed version of the following article: Hühn J, Carrillo-Carrion C, Soliman MG, et al. Selected Standard Protocols for the Synthesis, Phase Transfer, and Characterization of Inorganic Colloidal Nanoparticles. Chemistry of Materials. 2017;29(1):399-461 , which has been published in final form at 10.1021/acs.chemmater.6b04738. This article may be used for non-commercial purposes in accordance with ACS Terms and Conditions for Self-Archiving. 
Jonas Hühn ${ }^{1}$, Carolina Carrillo-Carrion ${ }^{2}$, Mahmoud G. Soliman ${ }^{1}$, Christian Pfeiffer ${ }^{1}$, Daniel Valdeperez ${ }^{1}$, Atif Masood ${ }^{1}$, Indranath Chakraborty ${ }^{1}$, Lin Zhu ${ }^{1}$, Marta Gallego ${ }^{2}$, Yue Zhao ${ }^{1}$, Monica Carril ${ }^{2,3}$, Neus Feliu' ${ }^{1}$, Alberto Escudero ${ }^{1,4}$, Alaaldin M. Alkilany ${ }^{1,5}$, Beatriz Pelaz ${ }^{1}$, Pablo del Pino ${ }^{1}$, Wolfgang J. Parak ${ }^{1,2^{*}}$

${ }^{1}$ Fachbereich Physik, Philipps Universität Marburg, Marburg, Germany

${ }^{2} \mathrm{CIC}$ biomaGUNE, San Sebastian, Spain

${ }^{3}$ Ikerbasque, Basque Foundation for Science, Bilbao, Spain

${ }^{4}$ Instituto de Ciencia de Materiales de Sevilla. CSIC - Universidad de Sevilla. Seville, Spain.

${ }^{5}$ Faculty of Pharmacy, The University of Jordan, Amman, Jordan * corresponding author: wolfgang.parak@physik.uni-marburg.de

Selected Standard Protocols for the Synthesis, Phase Transfer, and Characterization of Inorganic Colloidal Nanoparticles 
Abstract

Introduction

Protocols

I. Synthesis of hydrophobic inorganic NPs in organic solvent

I.1. General considerations

1.2. Synthesis of hydrophobic Au NPs ( $\approx 4 \mathrm{~nm}$ or $7 \mathrm{~nm}$ core diameter)

1.3. Synthesis of hydrophobic Ag NPs ( $\approx 4 \mathrm{~nm}$ core diameter)

1.4. Synthesis of hydrophobic FePt NPs ( $\approx 4 \mathrm{~nm}$ core diameter)

I.5. Synthesis of hydrophobic $\mathrm{Fe}_{3} \mathrm{O}_{4} \mathrm{NPs}(\approx 4 \mathrm{~nm}$ core diameter)

I.6. Synthesis of hydrophobic Sn NPs ( $\approx 11 \mathrm{~nm}$ core diameter)

1.7. Synthesis of hydrophobic CdSe and CdSe/ZnS NPs $(\approx 2-10 \mathrm{~nm}$ core diameter)

1.8. Synthesis of hydrophobic CdS NPs ( $\approx 2 \mathrm{~nm}$ core diameter)

II. Synthesis of hydrophilic inorganic NPs in aqueous solution

II.1 General considerations

II.2 Synthesis of hydrophilic Au NPs ( $\approx 5-100 \mathrm{~nm}$ core diameter)

II.3 Synthesis of hydrophilic Ag NPs ( $\approx 15 \mathrm{~nm}$ core diameter)

II.4 Synthesis of rod-shaped Au NPs (variable size)

II.5 Synthesis of star-shaped Au NPs ( $\approx 70 \mathrm{~nm}$ diameter, distance from tip to tip)

III. Ligand exchange procedures for hydrophilic NPs maintaining their hydrophilic character

III.1 General considerations

III.2 Ligand exchange on citrate-capped Au NPs with bis(p-sulfonatophenyl)phenylphosphine

III.3 Ligand exchange on citrate-capped Au NPs with thiolated polyethyleneglycol (PEG)

IV. Ligand exchange procedures to transfer hydrophilic NPs from aqueous solution to organic solvent and thus to render them hydrophobic

IV.1 General considerations

IV.2 Ligand exchange on citrate-capped Au NPs with PEG-SH followed by phase transfer of PEG-capped Au NPs to chloroform containing dodecylamine 
IV.3 Ligand exchange on CTAB-capped Au NRs with PEG-SH followed by phase transfer of PEG-capped Au NPs to chloroform containing dodecylamine

IV.4 Phase transfer of star-shaped PEG-capped Au NPs to chloroform containing dodecylamine

V. Ligand exchange procedures to transfer hydrophobic NPs into aqueous solution and thus to render them hydrophilic

V.1 General considerations

V.2 Ligand exchange on dodecanethiol-capped Au NPs with 3mercaptopropionic acid or 11-mercaptoundecanoic acid

V.3 Phase transfer of dodecanethiol-capped Ag NPs from chloroform to water using 3-mercaptopropionic acid or 11-mercaptoundecanoic acid

VI. Polymer coating to transfer hydrophobic NPs into aqueous solution and thus to render them hydrophilic

VI.1 General considerations

VI.2Synthesis of the amphiphilic polymer: poly(isobutylene-alt-maleic anhydride)-graft-dodecyl, PMA-g-dodecyl

VI.3 Phase transfer of alkanethiol-capped NPs from chloroform to water using PMA-g-dodecyl polymer

VII. Purification of hydrophilic NPs

VII.1 General considerations

VII.2 Filtration, ultrafiltration, and dialysis

VII.3 Size exclusion chromatography

VII.4 Gel electrophoresis

VII.5 Ultracentrifugation

VIII. Characterization of NPs' structural properties

VIII.1 General comments about the composition of the NPs

VIII.2 Determination of NP concentrations by elemental analysis

VIII.3 Transmission electron microscopy of NP cores

VIII.4 Transmission electron microscopy of NPs including their organic shell

VIII.5 X-ray diffraction

VIII.6 Thermogravimetric analysis

VIII.7 Infrared spectroscopy

VIII.8 Nuclear Magnetic Resonance 
IX. Characterization of NPs' optical properties

IX.1 UV/vis absorption spectroscopy

IX.2 Determination of NP concentrations from absorption spectra

IX.3 Fluorescence spectroscopy

IX.4 Determination of quantum yields

$X$. Characterization of NPs' colloidal properties

X.1 UV/vis absorption spectroscopy

X.2 Dynamic light scattering

X.3 Laser Doppler anemometry

$\mathrm{X} .4 \mathrm{pH}$ titration

Conclusions

Acknowledgements

References

Appendix: Used symbols 


\title{
Selected Standard Protocols for the Synthesis, Phase Transfer, and Characterization of Inorganic Colloidal Nanoparticles
}

\begin{abstract}
Synthesis, characterization, and applications of colloidal nanoparticles have been a prominent topic of current research interests within the last two decades. Available reports in the literature that describe the synthesis of colloidal nanoparticles are abundant with various degrees of reproducibility and simplicity. Moreover, different methods for the characterization of colloidal nanoparticles' basic properties are employed, resulting in conflicting results in many cases. Herein, we describe "in detail" selected standard protocols for the synthesis, purification, and characterization of various types of colloidal inorganic nanoparticles including gold nanoparticles, silver nanoparticles, iron oxide nanoparticles, and quantum dots. This report consists of five main parts: The first and the second part are dedicated to describing the synthesis of various types of hydrophobic and hydrophilic nanoparticles in organic solvents and in aqueous solutions, respectively. The third part describes surface modification of nanoparticles with focus on ligand exchange reactions, to allow phase transfer of nanoparticles from aqueous to organic solvents and vice versa. The fourth and the fifth part describe various general purification and characterization techniques used to purify and characterize nanoparticles, respectively. Collectively, this contribution does not aim to cover all available protocols in the literature to prepare inorganic nanoparticles, but rather provides detailed synthetic procedures to important inorganic nanocrystals with full description of their purification and characterization process.
\end{abstract}




\section{Introduction}

Colloidal inorganic nanoparticles (NPs) gained an extensive interest recently due to their fascinating electronic, catalytic, optical, magnetic, and biological properties, which enable a novel material chemistry and a wide range of promising applications. Continuous development in understanding the fundamental physics, chemistry, and applications of these inorganic NPs originates from the availability of facile and reproducible synthetic protocols. The literature is abundant with large number of synthetic protocols, which focus typically on controlling the nanoparticle size and shape in addition to purity, crystallinity, stability, and monodispersity ${ }^{1}$. However, the preparation of high quality inorganic NPs is still challenging and needs fine-tuning. For example, the widely used Frens protocol fails to prepare large citrate-capped gold NPs $\left(>50 \mathrm{~nm}\right.$ ) with acceptable monodispersity ${ }^{2}$. Recent modification to the Frens method by adapting the seed-mediated approach, allowed for the synthesis of citrate-capped gold and silver NPs with excellent monodispersity and large diameter (up to $100 \mathrm{~nm}$ as described in section II) ${ }^{3,4}$. More importantly, the reproducibility of available reports is an issue in some cases, which may originate from highly sensitive systems and thus fluctuations of any variable, such as the source of some chemicals, may have a significant impact on the reaction products. Another reason for the observed irreproducibility is the unexpressed "critical details" and "tricks" in the published reports. With this in mind and based on our own experience, we aim in this contribution to compile reproducible, simple, and detailed synthetic protocols that enable the synthesis and characterization of high quality inorganic NPs. Accordingly, sections I \& II in this contribution focus on the synthesis of hydrophobic and hydrophilic inorganic NPs including gold, silver, iron oxide and quantum dots.

Usually "as prepared" NPs need further modification to control their hydrophilicity, hydrophobicity, colloidal stability, biocompatibility, biological recognition, and many other properties. Powerful surface modification reactions and strategies are available in the literature, including ligand exchange, ligand modification, polymer coating, silanization ${ }^{5,6}$, as well as controlled bioconjugation ${ }^{7}$. Due to the importance of surface functionalization, we describe ligand exchange reactions in three sections (sections III, IV and V) and a polymer coating strategy in section VI. The described ligand exchange reactions might be employed to control the properties of the NPs such as: i) improving the colloidal stability and interaction with biological compartments (e.g. ligand exchange of citrate-capped Au NPs with thiolated polyethylene glycol in section III); ii) facilitating the phase transfer of NPs from water to organic solvent (e.g. ligand exchange of citrate-capped Au NPs with dodecylamine/polyethylene glycol mix in section IV); iii) facilitating the phase transfer of NPs from organic solvent to water (e.g. ligand exchange of alkanethiol capped Au NPs with 3-mercaptopropionic acid in 
section V). Another universal strategy of surface functionalization is detailed in section $\mathrm{VI}$, in which a polymer coating is employed to change hydrophobic inorganic NPs into highly charged NPs rending their surface hydrophilic and allowing their transfer to the aqueous solution. It is worth to mention that the described surface functionalization strategies herein are general and can be applied to NPs of other materials, sizes and shapes.

Similar to the synthesis of organic small molecules or polymers, the preparation of inorganic NPs requires a careful post-synthesis purification to get rid of "impurities", which can be any left over reactants, free surfactant/capping agents, or undesired reaction products. The purification of NP solution is critical in many cases, and should be considered as a vital step. For example, free cationic surfactants in gold nanorods ( $\mathrm{Au} \mathrm{NRs)} \mathrm{suspensions} \mathrm{are} \mathrm{responsible} \mathrm{for} \mathrm{cellular} \mathrm{toxicity} \mathrm{and} \mathrm{in} \mathrm{first} \mathrm{order} \mathrm{not} \mathrm{the} \mathrm{Au}$ NRs themselves, highlighting the importance of using "purified" Au NRs solution prior to biological evaluations/applications ${ }^{8}$. Another example is the presence of free recognition ligands (unbound) in NP solution, which should compete with bound ligand on the NPs for interaction with the designated target, which complicates interpretation of results and may result in serious artifacts. Despite the clear importance of the purification process, it is often overlooked in the literature and only briefly described in many published reports. With this in mind we tried to focus on this important topic through designating a separate section that describe various methods of NP purification including filtration, centrifugation, dialysis, size exclusion chromatography, and electrophoresis (see section VII).

After synthesis and purification of NPs, proper characterization is essential to confirm the NPs' structural, photophysical, and colloidal properties, as detailed in sections VIII, $I X$, and $X$, respectively. Structural characterization of NPs involves probing the quantitative and qualitative composition of NPs, size and shape determination of inorganic core and organic shell using electron microscopy, analysis of NP crystallinity using X-ray diffraction (XRD), quantification of the organic shell weight percentage on single NPs using thermogravimetric analysis, and confirming the chemical structure of the organic shell on the surface of NPs using nuclear magnetic resonance (NMR) analysis and infrared spectroscopy.

Photophysical characterization of NPs is very important, since it is the basis of various applications. For example, plasmon absorption and scattering of Au NPs allow sensing, imaging, and analytical applications of these nanostructures. The fluorescence phenomenon of quantum dots (QDs) is the basis for their use in imaging, tracking, and cellular labeling applications. Considering the importance of careful evaluation to the photophysical properties of NPs, it is addressed separately in section IX, which includes 
characterization of Au NPs' optical absorption and determination of NP concentration based on their absorption spectra. Also fluorescence spectroscopy as tool to characterize QDs and to calculate their quantum yields is detailed with examples.

Characterization of the colloidal properties of NPs such as hydrodynamic diameter, using dynamic light scattering (DLS) and effective surface charge using laser Doppler anemometry is very critical and thus included in section $X$. For example, measuring hydrodynamic diameters is an effective tool to follow NP aggregation, dissolution, and interaction with proteins or other ligands. Moreover, evaluation of the effective surface charge of NPs can be employed to confirm specific surface functionalization, such as ligand exchange, polymer overcoating, and protein adsorption. In section $\mathrm{X}$, following NP aggregation as function of salt addition or the solution's $\mathrm{pH}$ is discussed and detailed with examples of gold and silver NPs with various surface chemistries. Finally, characterization of the acidity/basicity of NPs by determination of their $\mathrm{pK}_{\mathrm{a}}$ and number of acidic or basic groups on single NPs using $\mathrm{pH}$ titration and laser Doppler anemometry is described. 


\section{Synthesis and characterization of hydrophobic inorganic NPs in organic solvent}

\section{I.1. General considerations}

1.2. Synthesis of hydrophobic Au NPs ( $\approx 4 \mathrm{~nm}$ or $7 \mathrm{~nm}$ core diameter)

I.3. Synthesis of hydrophobic Ag NPs ( $\approx 4 \mathrm{~nm}$ core diameter)

1.4. Synthesis of hydrophobic FePt NPs ( $\approx 4 \mathrm{~nm}$ core diameter)

I.5. Synthesis of hydrophobic $\mathrm{Fe}_{3} \mathrm{O}_{4} \mathrm{NPs}(\approx 4 \mathrm{~nm}$ core diameter)

1.6. Synthesis of hydrophobic Sn NPs ( $\approx 11 \mathrm{~nm}$ core diameter)

I.7. Synthesis of hydrophobic CdSe and CdSe/ZnS NPs ( $\approx 2-10 \mathrm{~nm}$ core diameter)

\subsection{Synthesis of hydrophobic CdS NPs ( $\approx 2 \mathrm{~nm}$ core diameter)}

\section{I.1. General considerations}

All glassware was first cleaned with detergent followed by aqua regia and carefully rinsed with Milli-Q water and acetone to guarantee an extremely cleaned glass surface. Aqua regia oxidizes and dissolves residual organic and inorganic impurities, which may interfere with the synthesis of NPs. Aqua regia solution is prepared by mixing 3 volume parts of hydrochloric acid $(\mathrm{HCl})$ with 1 volume part of nitric acid $\left(\mathrm{HNO}_{3}\right)$ and should be used fresh. Aqua regia is corrosive and strong oxidizing agent that should be prepared with care in a well-ventilated fume cupboard with protective clothing, goggles, and gloves.

Subsections $1.2-1.8$ describe the detailed synthesis of various types of NPs (Au, Ag, $\mathrm{FePt}, \mathrm{Fe}_{3} \mathrm{O}_{4}, \mathrm{Sn}, \mathrm{CdSe}, \mathrm{CdSe} / \mathrm{ZnS}$, and $\mathrm{CdS}$ ) in hydrophobic organic solvents. In these syntheses, the inorganic NP cores are stabilized by a shell of organic hydrophobic ligands (Figure I.1.1), which ensures colloidal stability of NPs in organic solvents such as toluene or chloroform. The effective NP diameter $\left(d_{\text {eff }}\right)$ is the sum of the inorganic core diameter $\left(d_{c}\right)$ and the thickness of the stabilizing ligand shell (ligand) as per Equation I.1.1. In case the NP core is comprised of $(n)$ layers of different materials (e.g. coreshell NPs), the diameter of the $1^{\text {st }}$ inner material is referred to $\left(d_{c 1}\right)$ and the diameter of the core after the $2^{\text {nd }}$ material is referred to $\left(d_{c 2}\right)$. The diameter of the core after the $n^{\text {th }}$ material is referred to $\left(d_{c 12 \ldots n}\right)$.

$$
\mathrm{d}_{\text {eff }}=\mathrm{d}_{\mathrm{c}}+2 \times \mathrm{l}_{\text {ligand }}
$$




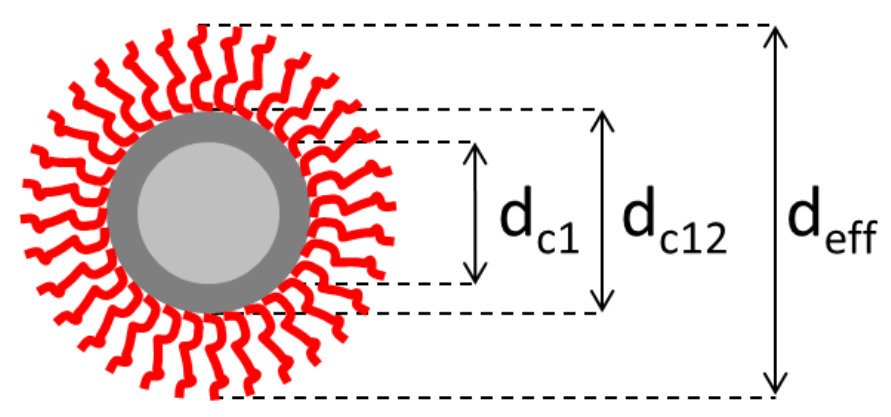

Figure I.1.1. The scheme shows an inorganic NP, which consists of an inorganic core and an organic hydrophobic ligand shell (drawn in red). The inorganic core here is composed of a core/shell structure of two different materials (first martial is the inner core drawn in light gray, and the second material is the inorganic shell drawn in dark gray).

\section{I.2. Synthesis of hydrophobic Au NPs ( $\approx 4 \mathrm{~nm}$ or $7 \mathrm{~nm}$ core diameter)}

Dodecanethiol-stabilized Au NPs ( $\approx 4 \mathrm{~nm}$ core diameter): Hydrophobic Au NPs were synthesized according to the Brust-Schiffrin two-phase-method ${ }^{9}$, with some modifications, as previously published ${ }^{10}$. The reaction was carried out at room temperature (RT) under ambient conditions. An aqueous phase with gold precursor (a yellow translucent solution containing $\mathrm{AuCl}_{4}{ }^{-}$ions) was prepared by dissolving $300 \mathrm{mg}$ (0.9 mmol) of hydrogen tetrachloroaurate (III) (99.9\%, Alfa Aesar \#12325) in $25 \mathrm{~mL}$ of Milli-Q water. An organic phase containing $2.170 \mathrm{~g}(3.9 \mathrm{mmol})$ tetraoctylammonium bromide (TOAB, $\mathrm{N}\left(\mathrm{C}_{8} \mathrm{H}_{17}\right)_{4}{ }^{+} \mathrm{Br}^{-}$, Sigma-Aldrich \#29,413-6) dissolved in $80 \mathrm{~mL}$ of toluene (Fluka 89682) was prepared immediately afterwards.

The two solutions, i.e. the aqueous and organic phases, were mixed in a $500 \mathrm{~mL}$ separation funnel (cf. Figure I.2.1 and Figure I.2.2), and shaken vigorously for about 5 min. Upon mixing, $\mathrm{AuCl}_{4}{ }^{-}$ions (in the aqueous yellow phase) were gradually transferred into the organic toluene phase, forming tetraoctylammonium tetrachloroaurate ion pairs $\left(\mathrm{N}_{(}\left(\mathrm{C}_{8} \mathrm{H}_{17}\right)_{4}{ }^{+} \mathrm{AuCl}_{4}{ }^{-}\right)$as per Equation I.2.1. Within this process, the initial colorless toluene phase (tol) turned into a deep orange color, while the initial yellow color of the aqueous phase (aq) turned colorless (see Figure I.2.2.A-C).

$$
\mathrm{HAuCl}_{4}{ }^{-}(\mathrm{aq})+\mathrm{N}\left(\mathrm{C}_{8} \mathrm{H}_{17}\right)_{4}{ }^{+} \mathrm{Br}^{-} \text {(tol) } \rightarrow \mathrm{N}\left(\mathrm{C}_{8} \mathrm{H}_{17}\right)_{4}{ }^{+} \mathrm{AuCl}_{4}{ }^{-} \text {(tol) }+\mathrm{HBr} \text { (aq) (Equation I.2.1) }
$$

The aqueous solution (at the bottom) was discarded and the organic phase containing gold precursors was transferred to a $250 \mathrm{~mL}$ round flask. 


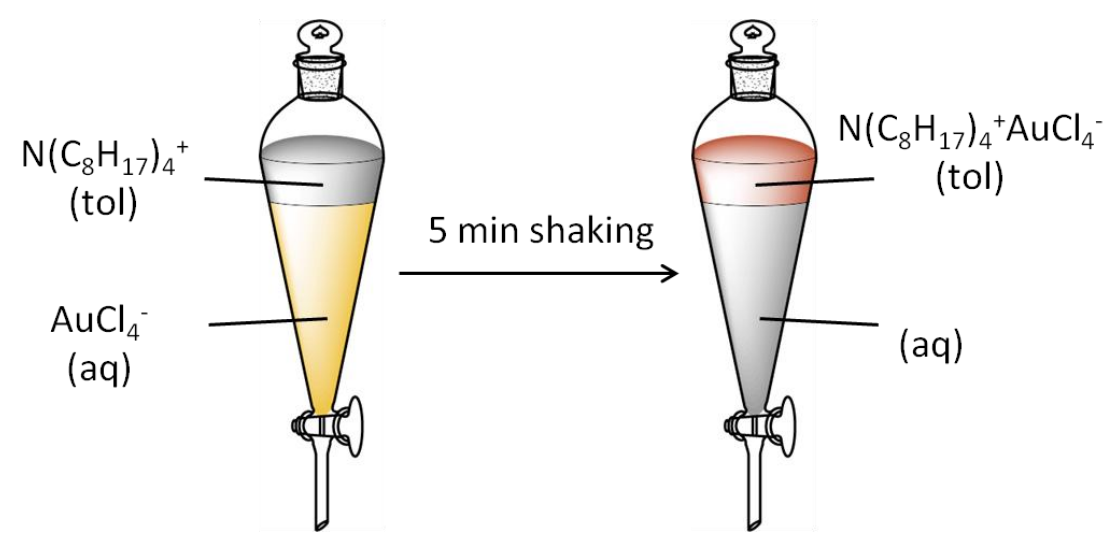

Figure I.2.1. Scheme of the process of transferring $\mathrm{AuCl}_{4}^{-}$ions from the aqueous phase to the organic phase through the formation of tetraoctylammonium tetrachloroaurate ion pairs.

A

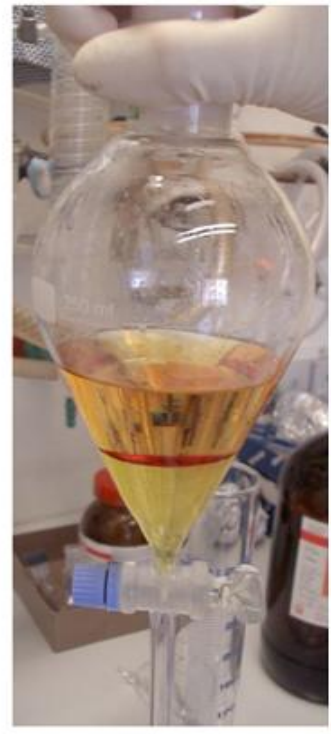

B

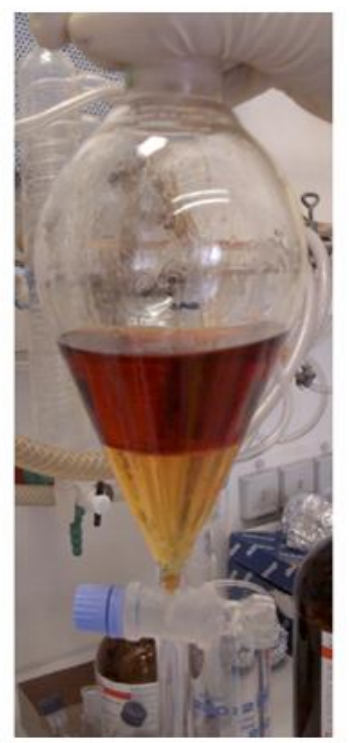

C

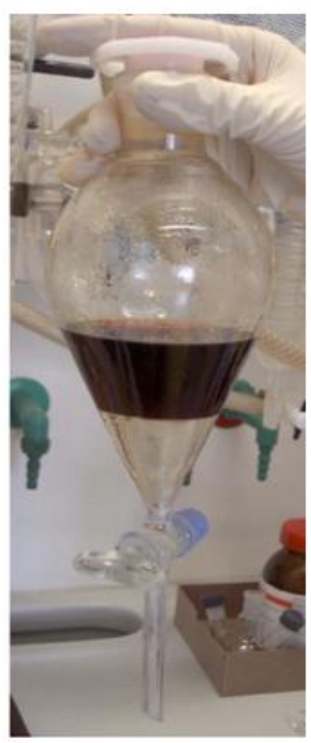

Figure I.2.2. Photographs of the biphasic system showing the process of transferring $\mathrm{AuCl}_{4}^{-}$ions from the aqueous phase (lower phase) to the organic phase (upper phase) through the formation of tetraoctylammonium tetrachloroaurate ion pairs (dark red phase in C).

In addition to the gold precursor solution, a solution with a strong reducing agent was prepared as follow: In a beaker, $334 \mathrm{mg}$ of sodium borohydride $\left(\mathrm{NaBH}_{4} 98 \%\right.$, SigmaAldrich \#452882) were dissolved in $25 \mathrm{~mL}$ of Milli-Q water under vigorous stirring. The prepared solution should be used immediately after preparation due to the fast decomposition of sodium borohydride as per Eq. I.2.2. Observing small bubbles due to the formation of hydrogen is common. 


$$
\mathrm{BH}_{4}^{-}(\mathrm{aq})+3 \mathrm{H}_{2} \mathrm{O} \rightarrow \mathrm{B}(\mathrm{OH})_{3}(\mathrm{aq})+3 \mathrm{H}_{2}(\uparrow)+\mathrm{H}^{+}+\mathrm{e}^{-}
$$

In order to obtain Au NPs, the freshly prepared solution of sodium borohydride was added drop-wise within one minute to the vigorously stirred organic phase containing the gold precursor (see Figure I.2.3 and Figure 1.2.4). Hereby the Au(III) precursor is reduced to $A u(0)$ forming $A u$ NPs. A few seconds after the addition of borohydride the color changed from deep orange (Au(III) precursor) to red-violet (tetraoctylammonium bromide capped Au NPs, TOAB-Au NPs).

$$
\mathrm{n} \times \mathrm{Au}^{3+}(\text { tol })+3 \mathrm{n} \times \mathrm{e}^{-} \rightarrow \mathrm{Au}_{\mathrm{n}}
$$

The solution was kept stirring for about $1 \mathrm{~h}$ in order to reduce remaining gold ions (Figure 1.2.4).

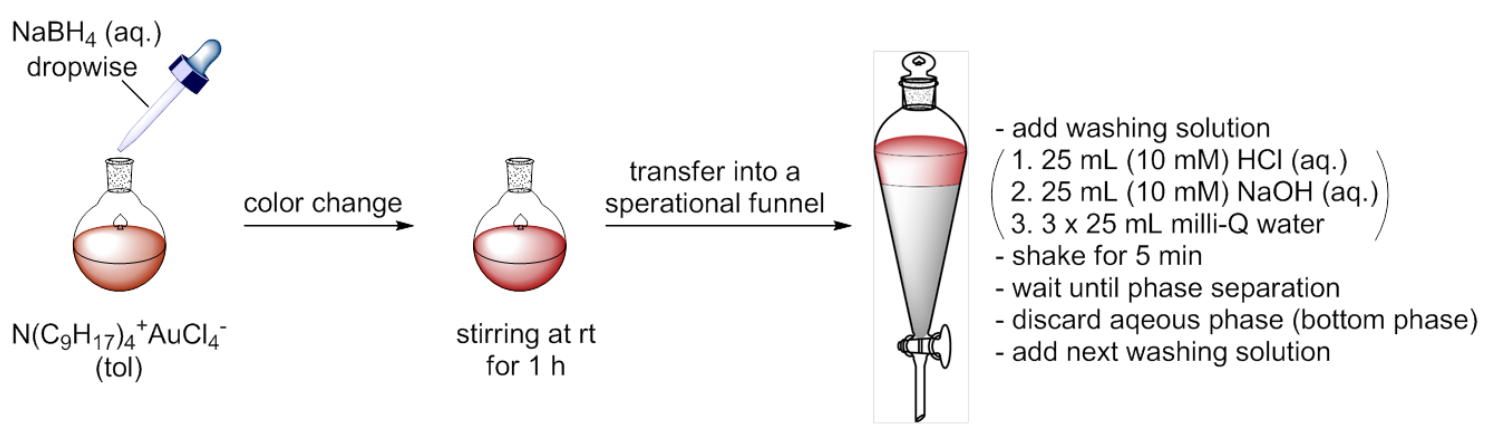

Figure I.2.3. The synthesis of $4 \mathrm{~nm} T O A B-A u$ NPs is based on the reduction of $A u(I I I)$ to elemental $\mathrm{Au}(\mathrm{O})$ by the addition of $\mathrm{NaBH}_{4}$. After the formation of Au clusters, the NP suspension is washed several times with aqueous solutions.

A

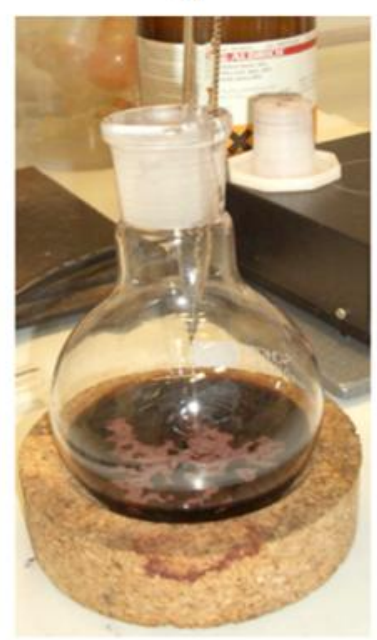

B

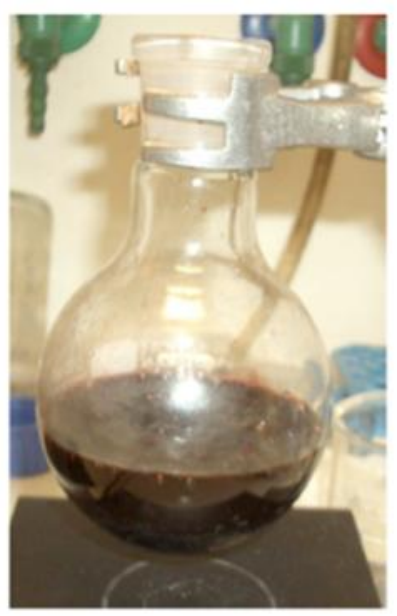

Figure I.2.4. Reduction process of gold ions by sodium borohydride. 
After $1 \mathrm{~h}$ of stirring at RT, the mixture was transferred into a separation funnel. The aqueous phase was discarded and the organic phase was washed with $25 \mathrm{~mL}$ of $10 \mathrm{mM}$ $\mathrm{HCl}$ (aq.), $25 \mathrm{~mL}$ of $10 \mathrm{mM} \mathrm{NaOH}$ (aq.) and 3 times with $25 \mathrm{~mL}$ Milli-Q water (cf. Figure I.2.3). For the washing steps the solution used for washing (i.e. $\mathrm{HCl}, \mathrm{NaOH}$, or $\mathrm{H}_{2} \mathrm{O}$ ) was added in the funnel to the organic phase and the mixture was shaken. After separation of the organic and aqueous phase the aqueous phase at the bottom was discarded. Washing steps are necessary to get rid of residual precursors and salts.

A

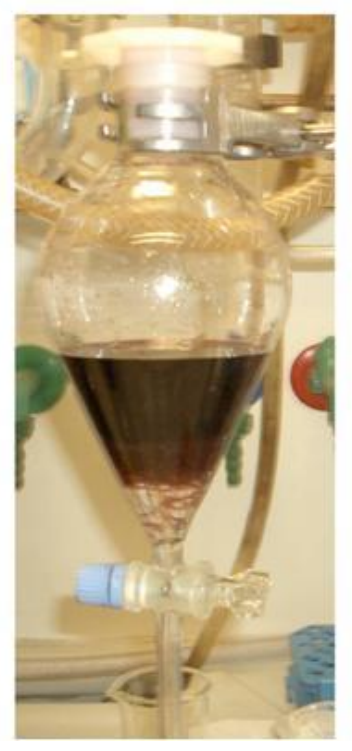

B

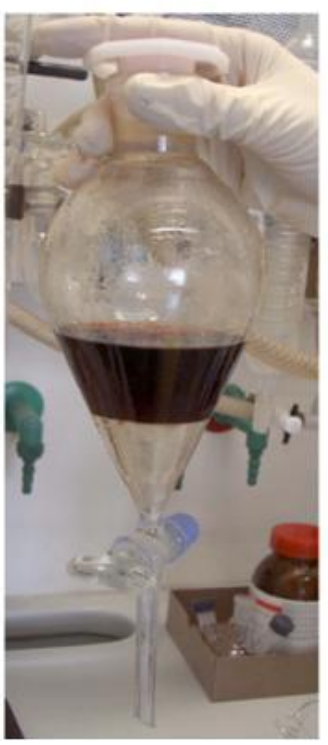

Figure I.2.5. Purification of TOAB-Au NPs. Upper toluene phase containing TOAB-Au $N P s$ is washed with aqueous solutions ( $\mathrm{HCl}, \mathrm{NaOH}$ and Milli-Q water) in the lower phase to remove excess sodium borohydride and other residual chemicals. A) Photograph of the two phases immediately after shaking note that the two phases are not completely separated. B) Photograph of the two phases after they were allowed to separate forming a transparent aqueous phase at the bottom and a deep red organic phase at the top containing TOAB-Au NPS.

After discarding the aqueous phase from the last washing step, the remaining deep red, organic solution (ca. $80 \mathrm{~mL}$ ) was transferred to a $250 \mathrm{~mL}$ round bottom flask and stirred overnight to get thermodynamically stable NPs with narrow size distribution, mediated by Ostwald ripening ${ }^{11-13}$ (cf. Figure I.2.6). 


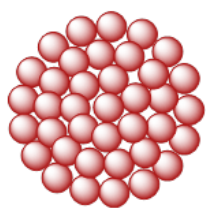

bigger Au NP

Transfer of $\mathrm{Au}(0)$ atoms from smaller Au NPs to bigger Au NPs.

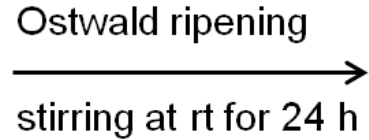

Homogeneous sized Au NPs of about $4 \mathrm{~nm}$ core diameter.

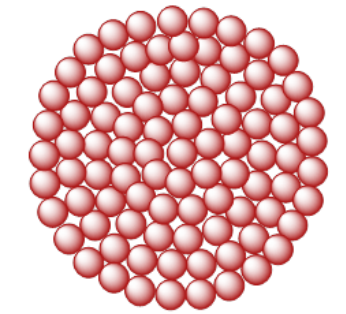

smaller Au NP

Figure I.2.6. Ostwald ripening phenomena results in TOAB-Au NPs with a narrow size distribution. Smaller Au NPs in inhomogeneous population in terms of size dissolve and end up in bigger Au NPs.

After the synthesis of TOAB-Au NPs, a ligand exchange procedure was carried out, in order to obtain dodecanethiol-stabilized Au NPs (DDT-Au NPs). TOAB-Au NPs are stabilized by weakly adsorbed TOAB molecules at the surface of Au NPs, which can be desorbed by various stresses, such as dilution or repeated centrifugation. In order to get more stable Au NPs, TOAB can be exchanged with alkanethiols (e.g. dodecanethiol) that assemble strongly on the surface of gold due to the strong Au-S bond ${ }^{14,15}$.

For the ligand exchange procedure $10 \mathrm{~mL}$ of 1-dodecanethiol (DDT, 98\%, Sigma-Aldrich $\# 47,136-4$ ) were added to the TOAB-Au NPs in toluene (ca. $80 \mathrm{~mL}$ ) (Figure 1.2 .7 and Figure I.2.8). The solution was heated to $65^{\circ} \mathrm{C}$ and stirred for $2 \mathrm{~h}$ to facilitate the ligand exchange and finally stirred at RT to cool down. During this process, the mercapto group in dodecanethiol molecules displaces the TOAB ${ }^{16}$, yielding dodecanethiolstabilized Au NPs. 


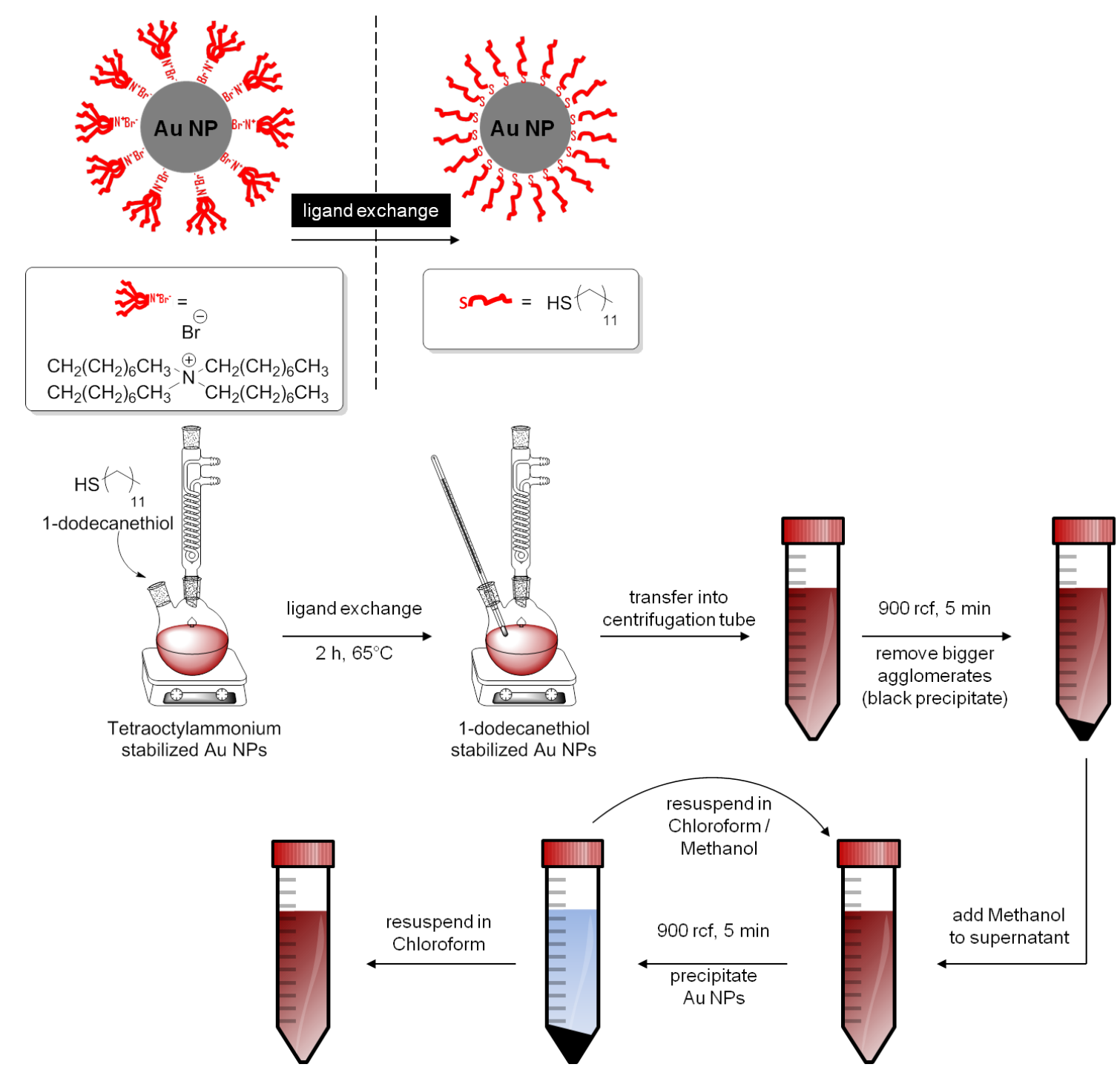

Figure I.2.7. Ligand exchange on TOAB-Au NPs with dodecanethiol leading to the formation of DDT-stabilized Au NPs. 


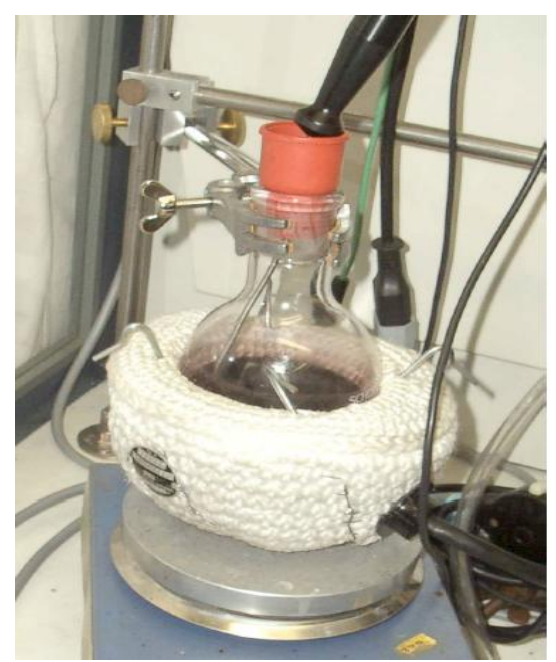

Figure 1.2.8. Au NPs are stirred after the addition of DDT while the temperature is controlled by a thermometer that is embedded in the reaction flask.

The solution of DDT-Au NPs was poured into several $40 \mathrm{~mL}$ vials and centrifuged at $900 \mathrm{rcf}$ for $5 \mathrm{~min}$, in order to remove larger agglomerates. The supernatant containing the well-dispersed DDT-Au NPs was collected and pooled, and the agglomerates (i.e. the precipitate) were discarded. DDT-Au NPs were then precipitated using methanol (99.8\%, Sigma, \#32,241-5) as a non-solvent. For this, about $20 \mathrm{~mL}$ of DDT-Au NPs solution was placed in $40 \mathrm{~mL}$ glass vials, followed by the gradual addition of methanol until the solution turned cloudy. The solution was then centrifuged at $900 \mathrm{rcf}$ for $5 \mathrm{~min}$, in order to precipitate the DDT-Au NPs. After this step of centrifugation, the supernatant turned colorless and DDT-Au NPs were collected on the bottom of the centrifugation tube. In case of incomplete collection of DDT-Au NPs, additional methanol can be added, in order to increase the polarity of the medium and thus promoting the precipitation of the DDT-Au NPs, cf. Figure 1.2.9. After discarding the supernatant (which contains excess DDT and the replaced TOAB), the collected DDT-Au NPs were resuspended in chloroform. Similarly, DDT-Au NPs in chloroform were precipitated by the addition of methanol (enough amount to induce turbidity), followed by centrifugation for $5 \mathrm{~min}$ at $900 \mathrm{rcf}$ to precipitate and collect DDT-Au NPs. After discarding the supernatant, collected DDT-Au NPs were resuspended in chloroform, leading to the final solution of DDT-Au NPs.

The described cleaning procedure by the addition of methanol (i.e. non-solvent) narrows the size distribution of the DDT-Au NPs. Upon the addition of only a little methanol bigger Au NPs precipitate, whereas the smaller Au NPs remain in the supernatant. This size selective precipitation allows for collecting NPs with similar sizes and discarding extremely small NPs (Figure 1.2 .9$)^{17}$. It is worth to note here that the use of excess methanol should be avoided, since this will coprecipitate excess ligand molecules in solution along with the collected NPs, and thus hinder the purification ${ }^{18}$. 


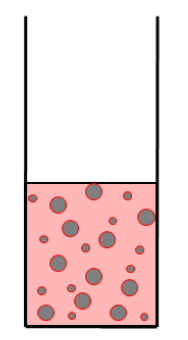

NPs dispersed in tolune

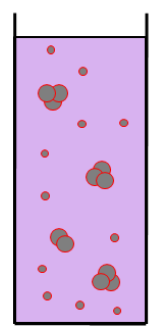

Mixture is more polar, NPs aggregate

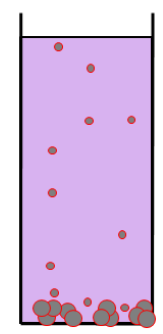

Aggregates can be precipitated in centrifuge
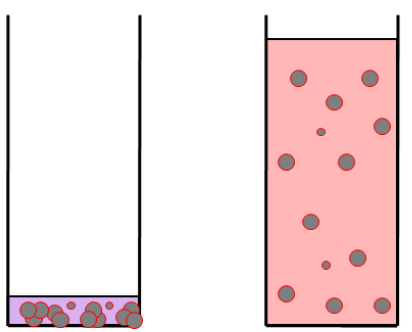

Supernatant taken off
Fresh nonpolar solvent is added

Figure I.2.9. Size selective precipitation method to purify DDT-Au NPs and narrow their particle size distribution. The addition of a polar non-solvent (methanol or acetone, drawn in blue) to DDT-Au NPs in hydrophobic solvent (e.g. toluene or chloroform, drawn in red) results in agglomeration and precipitation of large DDT-Au NPs. Collected precipitates of large DDT-Au NPs are redispersed in fresh hydrophobic solvent resulting in DDT-Au NPs with higher monodispersity. Smaller DDT-Au NPs remaining in supernatant are discarded ${ }^{17}$.

Synthesis of Au NPs in presence of hexanoic acid: Hexanoic acid-stabilized Au NPs $(\approx 4$ nm core diameter): In a $25 \mathrm{~mL}$ round bottom flask a solution of $29 \mu \mathrm{L}(231 \mathrm{nmol})$ hexanoic acid (Sigma Aldrich, \#153745) dissolved in $2 \mathrm{~mL}$ toluene, was mixed with $25 \mathrm{mg}$ (97.2 mmol) Tetrabutylammonium borohydride (TBAB, 98\%, Alfa Aesar, \#A17494) dissolved in $1 \mathrm{~mL}$ Didodecyldimethylammonium bromide (DDAB, 99\%, Alfa Aesar, \#B22839) stock solution (100 mM in toluene) at RT. In addition a solution of $500 \mu \mathrm{L}(25 \mu \mathrm{mol}) \mathrm{HAuCl}_{4}$ (Sigma Aldrich, \#254169) (50 mM in DDAB stock) and $2 \mathrm{~mL}$ DDAB stock were added fast under vigorous stirring. The solution was left for ripening for $1 \mathrm{~h}$ stirring at RT before further use.

Synthesis of Au NPs in presence of decanoic acid: Decanoic acid-stabilized Au NPs $(\approx 7$ nm core diameter): For this purpose, a solution of $43 \mathrm{mg}(250 \mu \mathrm{mol})$ decanoic acid (Sigma Aldrich, \#C1875) dissolved in $2.5 \mathrm{~mL}$ toluene, and a solution of $25 \mathrm{mg}$ (97.2 mmol) TBAB dissolved in $1 \mathrm{~mL}$ DDAB stock solution (100 $\mathrm{mM}$ in toluene) were added in a $25 \mathrm{~mL}$ flask. Then a solution of $500 \mu \mathrm{L}(25 \mu \mathrm{mol}) \mathrm{HAuCl}_{4}(50 \mathrm{mM}$ in DDAB stock) and $2 \mathrm{~mL} \mathrm{DDAB}$ stock were added to the mixture fast and under vigorous stirring and left for ripening for $1 \mathrm{~h}$ stirring at RT before further use.

It is worth to note that the synthesis of Au NPs in presence of hexanoic or decanoic acid results in weakly capped NPs with these ligands, which can be considered as 
"intermediate NPs" ready for further ligand exchange with thiol capped molecules. However, the use of these fatty acids ensures a narrow size distribution of the prepared Au NPs as described by Jana et al $^{19}$.

\section{I.3. Synthesis of hydrophobic Ag NPs ( $\approx 4 \mathrm{~nm}$ core diameter)}

Also silver NPs (Ag NPs) can be synthesized in organic solvents ${ }^{20}$. The herein described synthesis of Ag NPs is similar to the synthesis of Au NPs as described in §l.2. Mari et al. reported an effective protocol to prepare alkanethiol-stabilized Ag NPs $(\approx 4.2 \mathrm{~nm}$ in core diameter) ${ }^{21}$. The first step was the synthesis of an alkyl thiosulfate (namely sodium S-dodecylthiosulfate) as a ligand precursor as per Equation I.3.1.

$$
\mathrm{Na}_{2} \mathrm{~S}_{2} \mathrm{O}_{3} \cdot 5 \mathrm{H}_{2} \mathrm{O}+\mathrm{CH}_{3}\left(\mathrm{CH}_{2}\right)_{11} \mathrm{Br} \rightarrow \mathrm{CH}_{3}\left(\mathrm{CH}_{2}\right)_{11} \mathrm{~S}_{2} \mathrm{O}_{3}{ }^{-} \mathrm{Na}^{+}+\mathrm{NaBr}+5 \mathrm{H}_{2} \mathrm{O} \text { (Equation I.3.1) }
$$

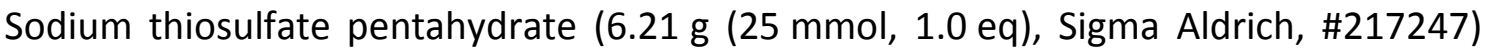
was dissolved in $50 \mathrm{~mL}$ Milli-Q water. In addition, $5.19 \mathrm{~mL}$ (25 mmol, $1.0 \mathrm{eq})$ 1-bromododecane (Sigma Aldrich, \#B65551) were dissolved in $50 \mathrm{~mL}$ ethanol. The combined solutions were stirred under reflux for $3 \mathrm{~h}$. The mixture was cooled down to RT and the resulting crystals of sodium $S$-dodecylthiosulfate were filtered using a funnel with a proper filter paper. Another crystallization step was performed, where sodium $S$-dodecylthiosulfate was dissolved in as less ethanol as possible under heating $\left(\sim 45^{\circ} \mathrm{C}\right)$ and left in a crystallization beaker for cooling to RT. In this manner, purification of the product was obtained by allowing the solution to gradually cool and be saturated with the compound. The product of interest crystallized and unwanted contaminations remained in the liquid phase. Finally, the sodium S-dodecylthiosulfate crystals were filtered as previously described and the final product was dried in vacuum (cf. Figure I.3.1). Importantly, the synthesis should be done in a fume hood and under controlled laboratory conditions. 


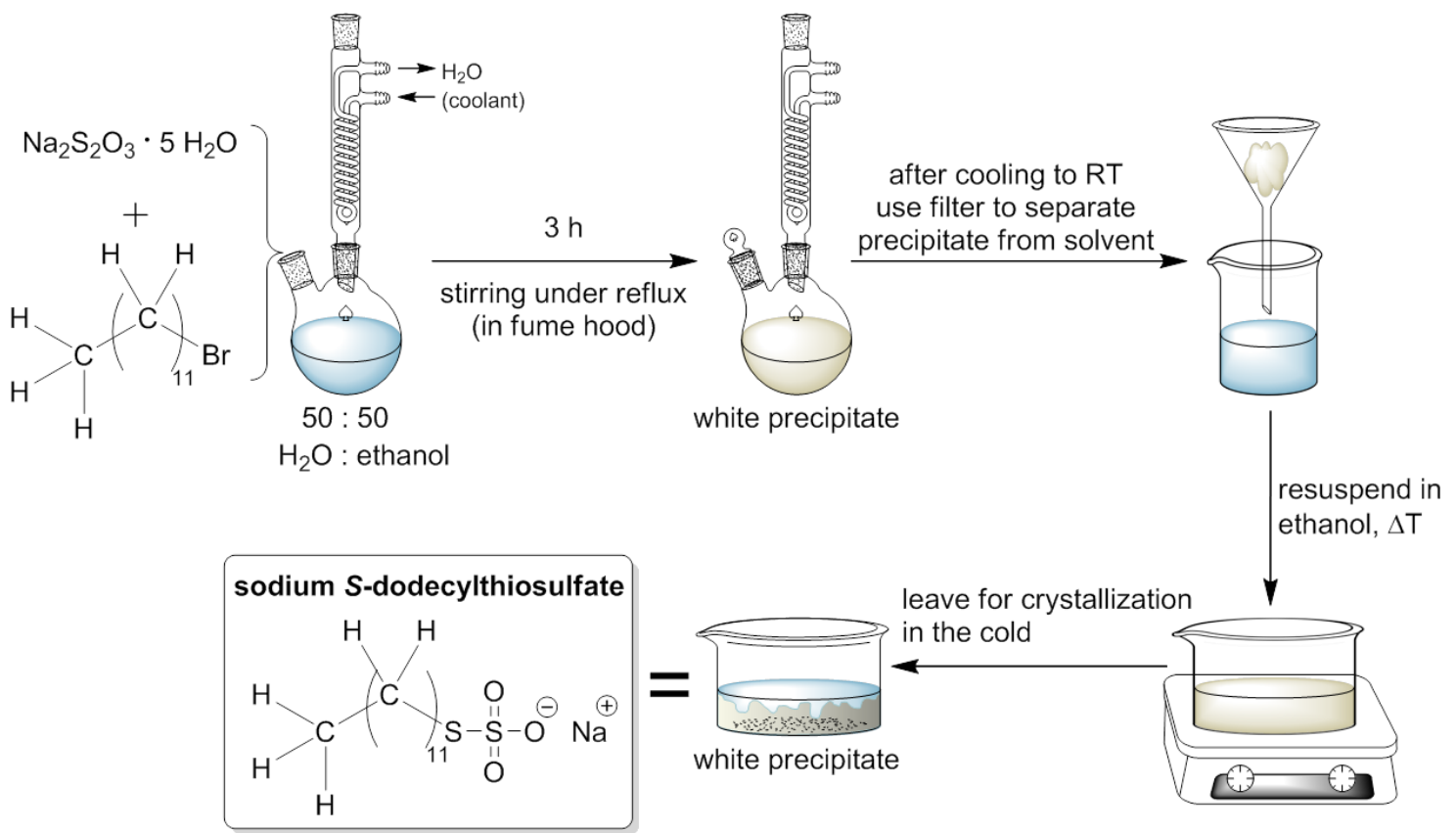

Figure 1.3.1. A schematic illustration of the chemical synthesis of sodium S-dodecylthiosulfate as a ligand precursor to stabilize Ag NPs.

For the synthesis of Ag NPs, $390 \mathrm{mg}(1.26 \mathrm{mmol}, 0.75 \mathrm{eq})$ sodium S-dodecylthiosulfate in $90 \mathrm{~mL}$ ethanol were heated up to $50{ }^{\circ} \mathrm{C}$. To this solution $282 \mathrm{mg}(1.68 \mathrm{mmol}, 1.0 \mathrm{eq})$ $\mathrm{AgNO}_{3}$ (Sigma Aldrich, \#209139) were added, and the resulting mixture was stirred for further $10 \mathrm{~min}$. A color change was observed, the white solution turned brown after a few minutes (cf. Figure I.3.2). $318 \mathrm{mg}(8.4 \mathrm{mmol}, 5.0 \mathrm{eq})$ sodium borohydride $\left(\mathrm{NaBH}_{4}\right.$ $98 \%$, Sigma-Aldrich, \#452882) were dissolved in $15 \mathrm{~mL}$ ethanol and added to the mixture, which turned almost black immediately (cf. Figure 1.3.2). After $5 \mathrm{~min} 74 \mathrm{mg}$ (0.42 mmol, $0.25 \mathrm{eq}$ ) ascorbic acid (Sigma Aldrich, \#255564) were added, and the mixture was stirred at $50{ }^{\circ} \mathrm{C}$ for further $3 \mathrm{~h}$. As the reaction mixture reached $\mathrm{RT}$, the solution with the Ag NPs was transferred into centrifuge tubes, and the Ag NPs were collected at the tube bottom by centrifuging at $3000 \mathrm{rcf}$ for $15 \mathrm{~min}$. After discarding the supernatant, the Ag NPs were resuspended in Milli-Q water and again collected by sedimentation ( $3000 \mathrm{rcf}, 15 \mathrm{~min}$ ). This washing step was repeated 2 more times using ethanol and then acetone ( $c f$. Figure 1.3.2). After that, the Ag NPs were dried under reduced pressure, which led to gray powder. For further use, the NPs were resuspended in chloroform. 

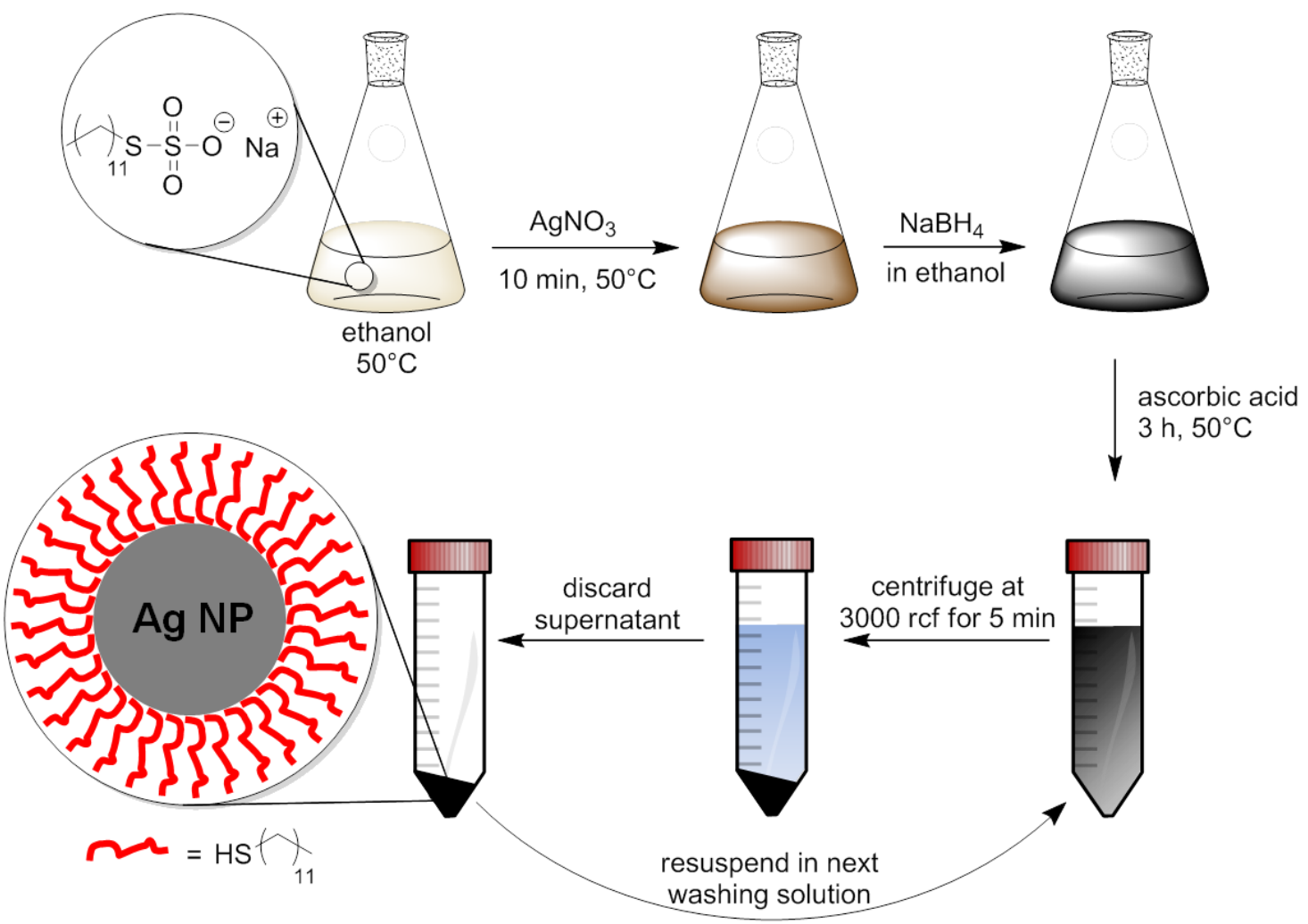

Figure 1.3.2. A graphic scheme of the synthesis and purification of dodecanethiolstabilized Ag NPs (DDT-Ag NPs).

\section{I.4. Synthesis of hydrophobic FePt NPs ( $\approx 4 \mathrm{~nm}$ core diameter)}

The synthesis of $\approx 4 \mathrm{~nm}$ core diameter FePt NPs as carried out here was described firstly by Sun et al. ${ }^{22}$ This reaction needs to be carried out in a water- and oxygen-free atmosphere (e.g. Schlenk line and/or glovebox). In a typical synthesis, platinum (II) acetylacetonate (197 mg, $0.5 \mathrm{mmol}, 1 \mathrm{eq}, A B C R, \# A B$ 121416), 1,2-hexadecanediol (390 mg, $1.5 \mathrm{mmol}, 3 \mathrm{eq}$, Sigma Aldrich, \#213748), and dioctylether (20 mL, Sigma Aldrich, \#249599) were mixed in a three-neck flask, a magnetic bar was added, and the mixture was heated to $100{ }^{\circ} \mathrm{C}$. The temperature was controlled by a temperature sensor immersed in the solution (cf. Figure 1.4.1). When the desired temperature was reached, the solution presented a light yellow color. Then, oleic acid (OA, $0.16 \mathrm{~mL}, 0.5$ mmol, 1 eq, Sigma Aldrich, \#O1008), oleylamine (OLA, $0.17 \mathrm{~mL}, 0.5 \mathrm{mmol}, 1 \mathrm{eq}$, Sigma Aldrich, \#O7805), and $\mathrm{Fe}(\mathrm{CO})_{5}(0.13 \mathrm{~mL}, 1 \mathrm{mmol}, 2 \mathrm{eq}$, Sigma Aldrich, \#481718) were added. After the addition of the Fe precursor the color of the solution turned to light brown. The reaction was heated under reflux $\left(297^{\circ} \mathrm{C}\right)$ for $30 \mathrm{~min}$ (cf. Figure I.4.1). At this temperature, the solution started to boil and some fumes were observed. Moreover, the color of the solution changed to deep brown, indicating the formation 
of NPs. After 30 min of heating, the reaction mixture was allowed to cool down to RT by removing of the heating source.

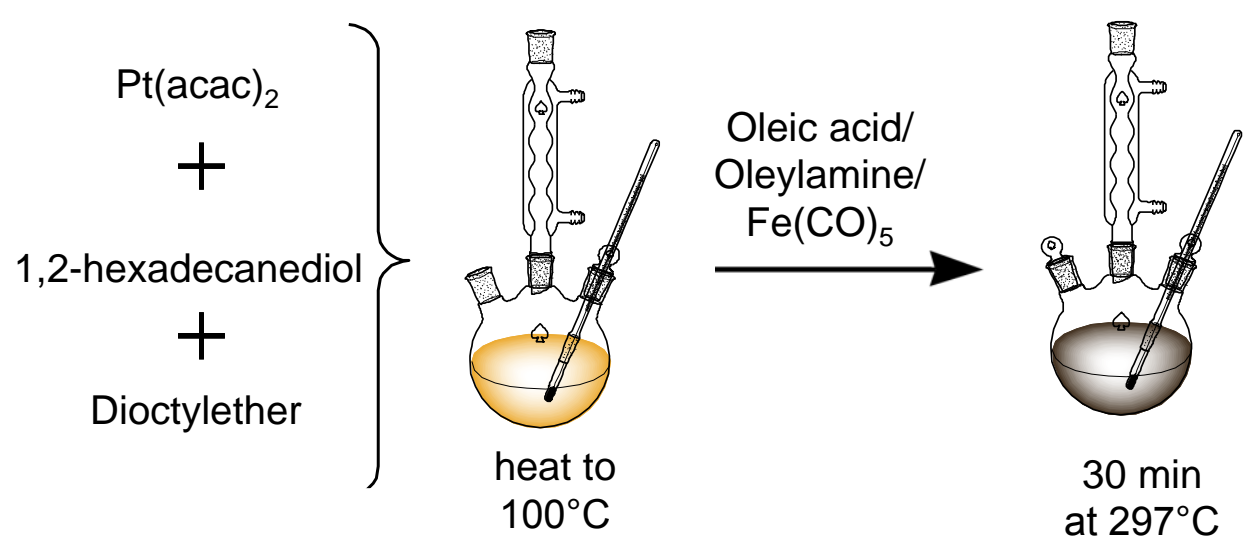

Figure I.4.1. Schematic illustration of the chemical synthesis of $4 \mathrm{~nm}$ core diameter FePt NPs. The synthesis was carried out under water exclusion and $\mathrm{N}_{2}$-atmosphere in a three neck flask equipped with seal septa to be able to add all reagents under oxygen and water free conditions.

For purification, the solution of FePt NPs was transferred to 2 centrifuge tubes of 50 $\mathrm{mL}$, and ethanol (40 mL, Carl Roth, \#64-17-5) was added to induce precipitation of the NPs. The NPs were separated by centrifugation (5 min, $2700 \mathrm{rcf}$ ) (cf. Figure 1.4.2). The brown colored supernatant containing byproducts was discarded and the black NP precipitate was dispersed in hexane $(25 \mathrm{~mL})$ in the presence of OA $(0.05 \mathrm{~mL})$ and OLA $(0.05 \mathrm{~mL})$, in order to provide the NPs colloidal stability for long storage periods.

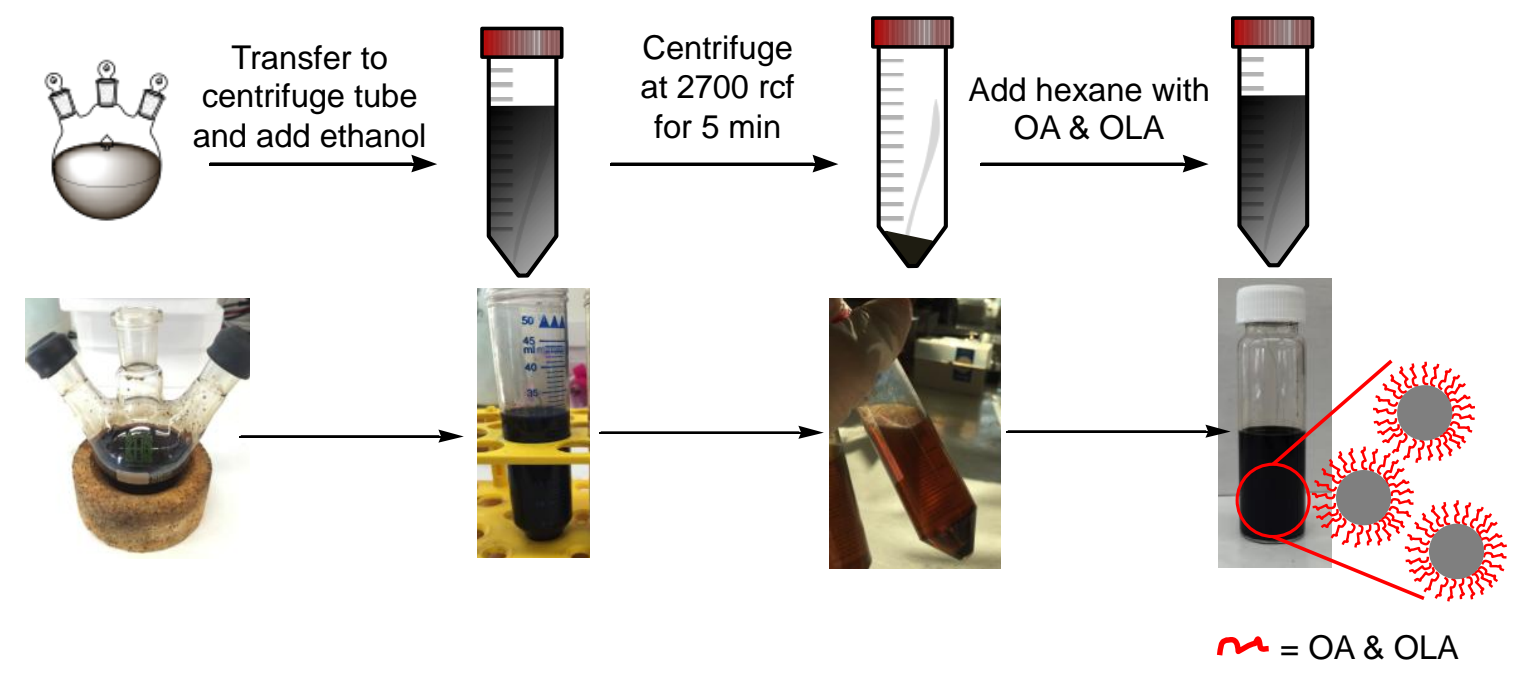

Figure I.4.2. Graphic scheme (upper panel) and photographs (lower panel) of FePt NPS purification steps after synthesis. 


\section{I.5. Synthesis of hydrophobic $\mathrm{Fe}_{3} \mathrm{O}_{4} \mathrm{NPs}(\approx 4 \mathrm{~nm}$ core diameter)}

The synthesis of $\mathrm{Fe}_{3} \mathrm{O}_{4}$ NPs as outlined here was initially published by Sun et al. in 2003 ${ }^{23}$, with a procedure to obtain monodisperse NPs with variable composition: $\mathrm{MFe}_{2} \mathrm{O}_{4}$, being $\mathrm{M}$ a metal that can be either iron, cobalt, or manganese, allowing to vary the magnetic properties. This protocol allows for the synthesis of NPs with a tunable diameter from 3 to $20 \mathrm{~nm}$ by changing the initial reaction conditions, or via a postsynthesis seed-mediated approach. In the present report only the synthesis of $4 \mathrm{~nm}$ core size $\mathrm{Fe}_{3} \mathrm{O}_{4} \mathrm{NPs}$ is presented and detailed (cf. Figure 1.5.1). To prepare these NPs, $706.4 \mathrm{mg}$ ( $2 \mathrm{mmol}, 1 \mathrm{eq}$ ) of the metal precursor iron (III) acetylacetonate (Fe(acac) 3 ; 99\%, Strem Chemicals, \#26-2300)) were dissolved in $20 \mathrm{~mL}$ diphenyl ether (SigmaAldrich, \#P24101) in the presence of $6 \mathrm{mmol}$ OA (3 eq, 90\%, Sigma-Aldrich, \#364525), $6 \mathrm{mmol}$ OLA ( $3 \mathrm{eq}$, Aldrich, \#O7805), and $10 \mathrm{mmol}$ 1,2-hexadecanediol (5 eq, Sigma Aldrich, \#52270). The process needs to be carried out in an oxygen- and water-free atmosphere (glovebox and/or Schlenk line), where the mixture was stirred at $200{ }^{\circ} \mathrm{C}$ for $30 \mathrm{~min}$. The mixture was then heated up to $265^{\circ} \mathrm{C}$ for another $30 \mathrm{~min}$ to induce thermal decomposition and thus the formation of NPs (cf. Figure I.5.1). The $\mathrm{Fe}_{3} \mathrm{O}_{4} \mathrm{NP}$ dispersion was then cooled down to RT and was taken away from the water- and oxygen-free ambient. In this preparation, 1,2-hexadecanediol acts as reducing agent to reduce the metal precursor into $\mathrm{Fe}_{3} \mathrm{O}_{4} \mathrm{NPs}$ that are stabilized with oleic acid and oleylamine. For purification, the dark brown dispersion was equally distributed into two $50 \mathrm{~mL}$ centrifuge tubes, then $25 \mathrm{~mL}$ of ethanol (Roth, \#5054.2) were added to each tube, and the NPs were centrifuged at $2700 \mathrm{rcf}$ for $5 \mathrm{~min}$. Supernatants were discarded and the $\mathrm{Fe}_{3} \mathrm{O}_{4} \mathrm{NPs}$ were collected at the bottom of the centrifugation vial. The $\mathrm{Fe}_{3} \mathrm{O}_{4}$ NPs were resuspended in a mixture of hexane with $1 \% \mathrm{v} / \mathrm{v}$ OA and OLA. Additional centrifugation at $2700 \mathrm{rcf}$ for $10 \mathrm{~min}$ is necessary to get rid of any undispersed residue/aggregates. The resulting supernatants were transferred to clean vials and a new washing step with ethanol was performed at $2700 \mathrm{rcf}$ for $5 \mathrm{~min}$ (cf. Figure I.5.1). The final $\mathrm{Fe}_{3} \mathrm{O}_{4} \mathrm{NPs}$ with a core diameter of $\approx 4 \mathrm{~nm}$ forming the pellet were finally redispersed into hexane supplemented with $1 \%$ OA and OLA. 

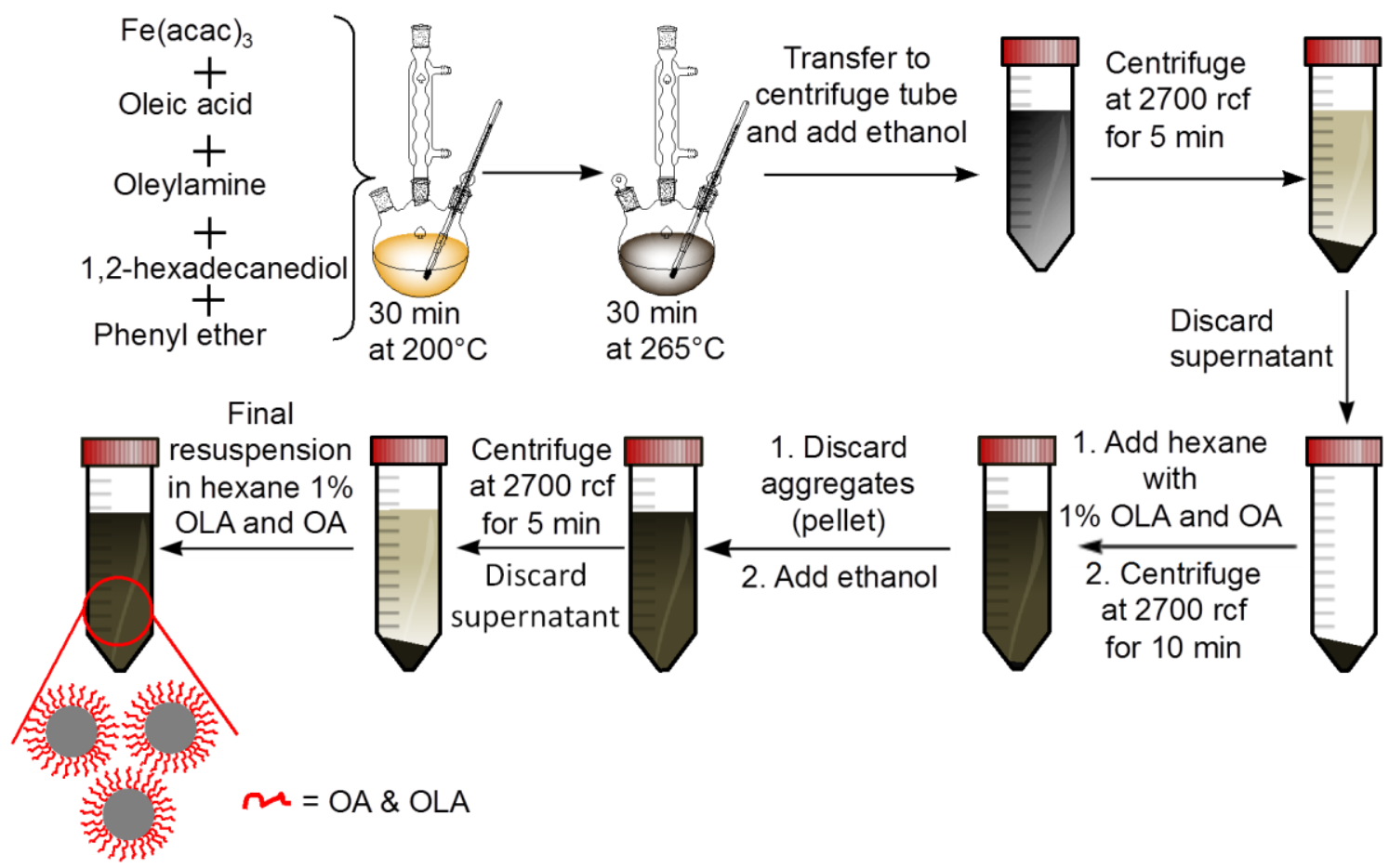

Figure 1.5.1. General scheme of the synthesis and purification of $\mathrm{Fe}_{3} \mathrm{O}_{4} \mathrm{NPs}$. After the initial synthesis, both, large aggregates and solvent impurities, are removed by centrifugation, obtaining NPs that are finally stabilized by OLA and OA.

\section{I.6. Synthesis of hydrophobic Sn NPs ( $\approx 11 \mathrm{~nm}$ core diameter)}

The synthesis of monodisperse Sn NPs with a core diameter of $11 \mathrm{~nm}$ as reported here was first published by Kravchyk et al. in $2013^{24}$. The protocol employs the reaction of $\mathrm{LiN}\left(\mathrm{Si}\left(\mathrm{CH}_{3}\right)_{3}\right)_{2}$ with a solution of oleylamine (OLA) and $\mathrm{SnCl}_{2}$ at $180{ }^{\circ} \mathrm{C}$, followed by the reduction of $\mathrm{Sn}$ (II) to elemental tin using diisobutylaluminum hydride (DIBAH) (Figure I.6.1). The reaction was completely performed inside a glove box under $\mathrm{N}_{2}$ atmosphere. Importantly, all glassware should be dried using heat and vacuum as the reaction is sensitive to water. The described reaction is violent and thus it should be carried out with caution, and safety regulations should be considered. 


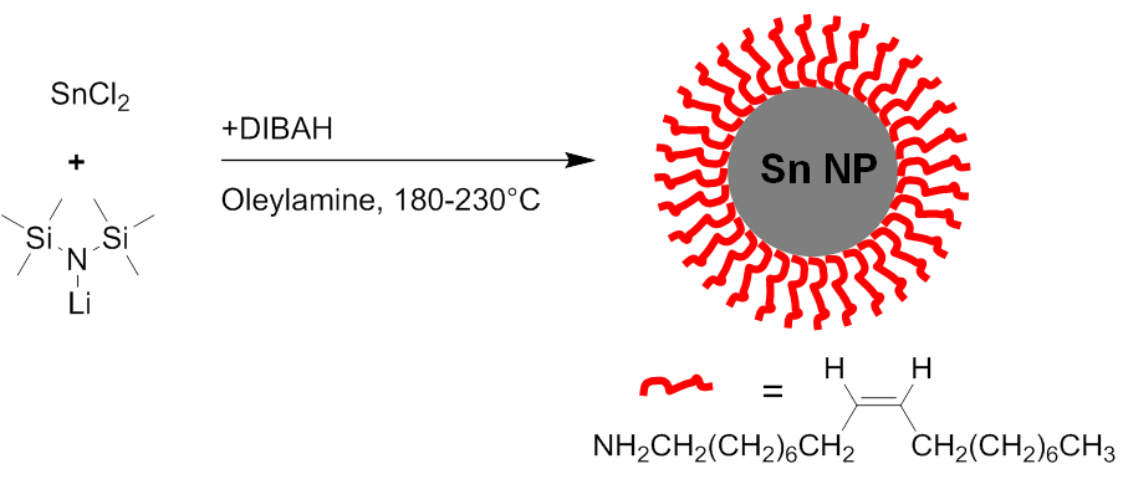

Figure I.6.1. Synthesis of monodispersed Sn NPs.

A $250 \mathrm{~mL}$ three neck flask was equipped with a reflux condenser, stirring bar, and two additional seal septa with one thermal sensor connected to a magnetic stirrer with heating capability. $24.6 \mathrm{~mL}$ OLA (Sigma Aldrich, $\geq 98 \%$, \#HT-OA100) were filled using a $20 \mathrm{~mL}$ syringe into the flask, and was stirred under vacuum at $140{ }^{\circ} \mathrm{C}$ for $1.5 \mathrm{~h}$. After cooling the solvent down to $50{ }^{\circ} \mathrm{C}, 94.8 \mathrm{mg}(0.5 \mathrm{mmol}, 1.0 \mathrm{eq}) \mathrm{SnCl}_{2}$ (Sigma Aldrich, \#208256) were added and the mixture was again dried under vacuum at $140{ }^{\circ} \mathrm{C}$ for $30 \mathrm{~min}$. Meanwhile $601 \mathrm{mg}$ (3.6 mmol, $7.2 \mathrm{eq}$ ) LiN(Si(CH$\left.)_{3}\right)_{2}$ (Sigma Aldrich, \#324620) were dissolved in $3 \mathrm{~mL}$ toluene using a small beaker. The mixture of OLA and $\mathrm{SnCl}_{2}$ was heated up to $180{ }^{\circ} \mathrm{C}$ under $\mathrm{N}_{2}$-atmosphere, followed by the injection of $\mathrm{LiN}\left(\mathrm{SiMe}_{3}\right)_{2}$ in toluene solution. After $10 \mathrm{~s}, 0.6 \mathrm{~mL}(0.6 \mathrm{mmol}, 1.2 \mathrm{eq})$ of a $1.0 \mathrm{M}$ solution of DIBAH dissolved in tetrahydrofuran (THF) (Sigma Aldrich, \#214981) was injected, which induced a color change to dark brown. The reaction mixture was further stirred for $1 \mathrm{~h}$ before putting the flask into an ice bath to cool it down. During this, at about $150{ }^{\circ} \mathrm{C}$, $10 \mathrm{~mL}$ toluene were added, and the flask was extracted out of the glove box and immediately held into an ice bath to speed up the cooling process (cf. Figure I.6.2). 


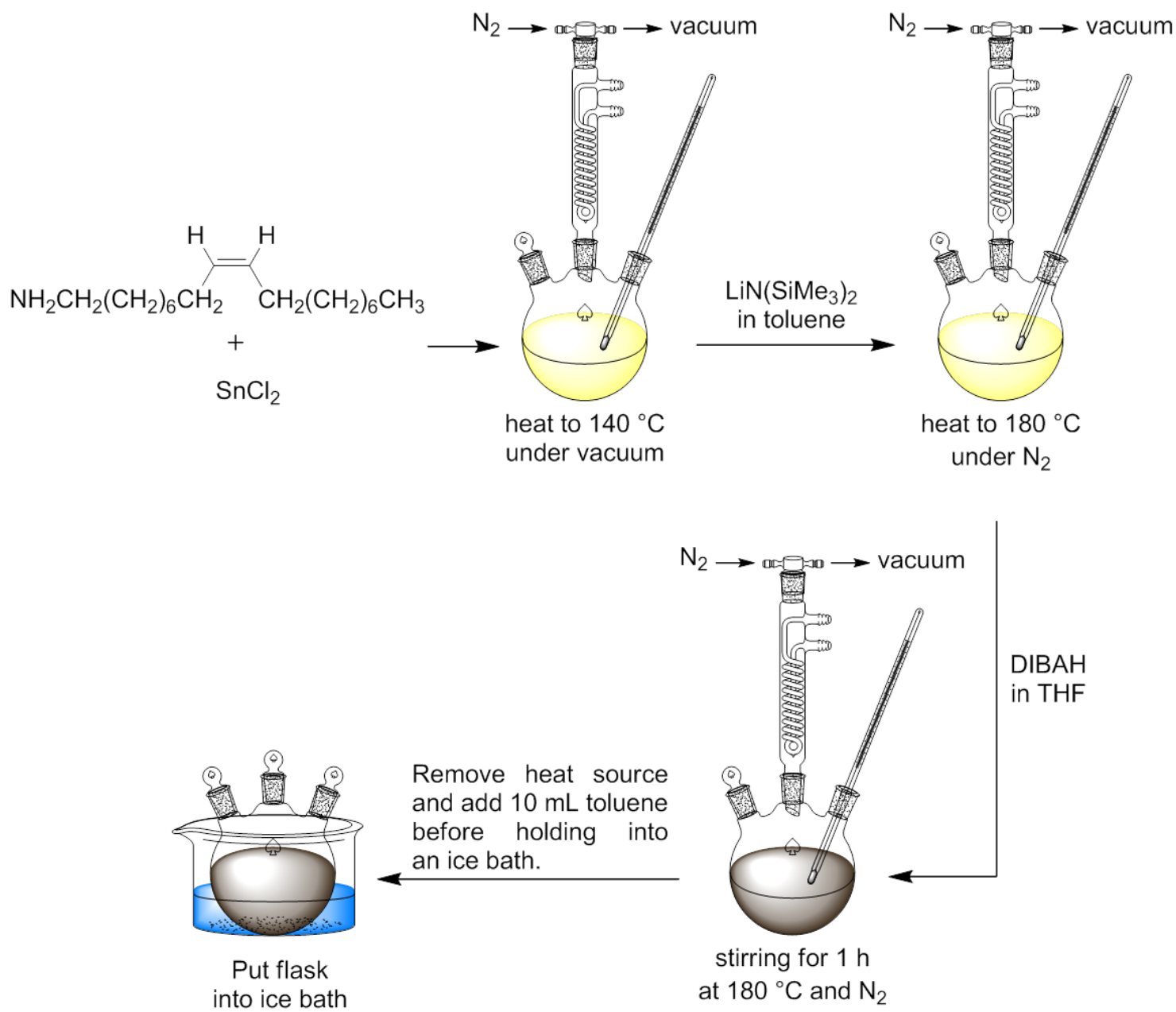

Figure 1.6.2. Schematic illustration of the chemical synthesis of $11 \mathrm{~nm}$ Sn NPs. The synthesis was carried out under water exclusion and $\mathrm{N}_{2}$-atmosphere in a three neck flask equipped with seal septa to be able of adding all reagents and guarantee oxygenand water-free conditions. For drying the solvent OLA and $\mathrm{SnCl}_{2}$ the reflux condenser was equipped with a three-way valve to be able to switch between $\mathrm{N}_{2}$ and vacuum. Before extracting the flask out of the glove box, the thermal sensor and reflux condenser were removed and all three necks were closed using seal septa.

When the Sn NP solution had cooled to RT it was transferred into two $50 \mathrm{~mL}$ centrifuge tubes and mixed with $40 \mathrm{~mL}$ ethanol, which led to precipitation of the Sn NPs. The sample was centrifuged at 3000 rcf for $5 \mathrm{~min}$, in order to separate the NPs from the solvent. The NPs were collected and the supernatant was discarded. Next, the Sn NPs were resuspended in a solution of oleic acid (OA) in chloroform ( $6 \mathrm{~mL}, 1 \mathrm{~mL} \mathrm{OA} / 50 \mathrm{~mL}$ $\mathrm{CHCl}_{3}$ ), which led to a ligand exchange from OLA to OA. Finally, a second precipitation step was performed by adding $10 \mathrm{~mL}$ ethanol to the solution and centrifugation at 3000 rcf for $5 \mathrm{~min}$ and resuspension in $10 \mathrm{~mL} \mathrm{CHCl} 3$ to generate a long term stable solution of Sn NPs (cf. Figure 1.6.3). 


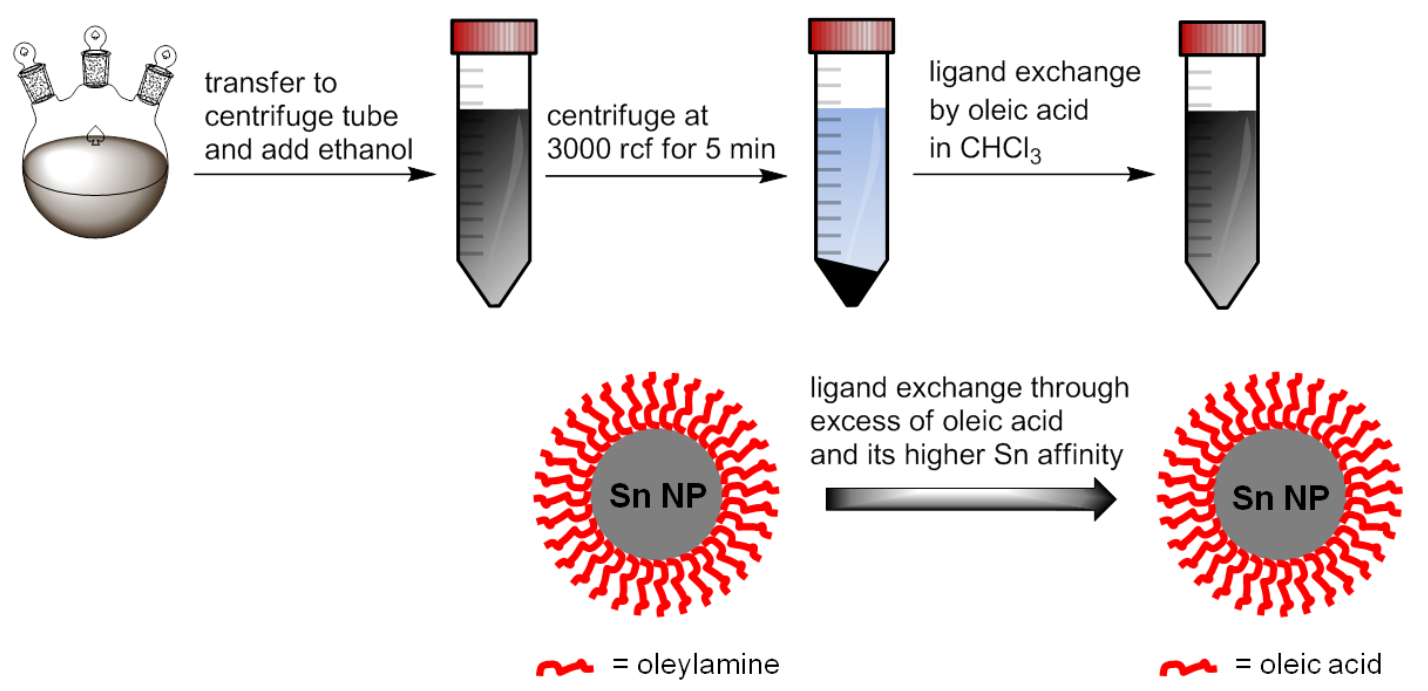

Figure I.6.3. Purification of Sn NPs and OLA ligand exchange with OA.

\section{I.7. Synthesis of hydrophobic CdSe and CdSe/ZnS NPs ( $\approx 2-10 \mathrm{~nm}$ core diameter)}

CdSe NPs, so called quantum dots (QDs), of different sizes were synthesized using CdO as a precursor, via the procedure described by Peng's group ${ }^{25}$, with minor modification in reaction temperature/time, in order to achieve a better control in the size of the NPs. To improve the quantum yield and long-term stability of these fluorescent NPs, coating of the CdSe NPs with a shell of ZnS was employed ${ }^{26}$ using diethylzinc and hexamethyldisilathiane as zinc and sulfur sources, respectively ${ }^{27}$.

Synthesis of CdSe cores: In a typical synthesis, $0.03 \mathrm{~g}$ of cadmium oxide (CdO, SigmaAldrich \#202894), $0.11 \mathrm{~g}$ of hexylphosphonic acid (HPA, Sigma-Aldrich \#750034), and $3.5 \mathrm{~g}$ of trioctylphosphine oxide (TOPO, Sigma-Aldrich \#223301) were loaded into a $100 \mathrm{~mL}$ glass three-neck flask connected to a nitrogen line (see Figure I.7.1). The mixture was degassed at $120{ }^{\circ} \mathrm{C}$ for $20 \mathrm{~min}$ and then heated to $300-320{ }^{\circ} \mathrm{C}$ under nitrogen atmosphere for 15 min to allow the complete dissolution of CdO in HPA and TOPO leading to a clear and colorless solution, which indicates that a Cd-HPA/TOPO complex has been formed. In case the CdO did not dissolve completely, (i.e. the solution did not turn transparent) some more HPA needs to be added. 


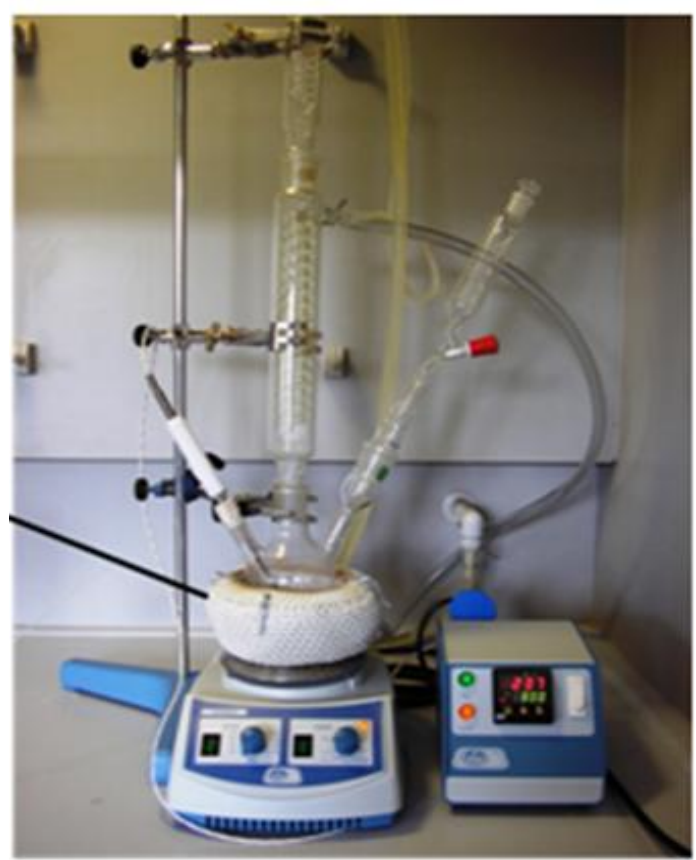

Figure I.7.1. Setup for the synthesis of CdSe/ZnS NPs. The photo shows a three-neck flask equipped with a heating mantle, a reflux condenser, an addition funnel, a thermometer connected to a temperature controller, and a nitrogen flow.

Then, the temperature of the solution was cooled down to $270{ }^{\circ} \mathrm{C}$ by setting the temperature controller to this temperature and waiting for around $10-15 \mathrm{~min}$. When the desired temperature was reached, $1.2 \mathrm{~mL}$ of selenium stock solution was swiftly injected with a syringe. The selenium stock solution was prepared by dissolving 0.0255 $\mathrm{g}$ of selenium powder (Se, Sigma-Aldrich \#229865) in $1.5 \mathrm{~mL}$ of trioctylphosphine (TOP, Sigma-Aldrich \#718165), to produce a $0.215 \mathrm{M}$ stock solution of trioctylphosphine selenide (Se-TOP). After injection, the temperature dropped by roughly $20^{\circ} \mathrm{C}$ and was maintained then at $250{ }^{\circ} \mathrm{C}$ throughout the synthesis of the CdSe cores. $3-5$ min after the injection the color of the solution turned from colorless to yellow, indicating the nucleation of CdSe NPs. Upon further grown of the NPs this color gradually turned to yellow-orange, orange, red and finally dark red 30-40 min after the injection.

CdSe NP growth was monitored by taking aliquots from the reaction flask every 5 min. Aliquot solutions were diluted with chloroform and their UV/visible absorption spectra were recorded (cf. Figure 1.7.2). Even when the growth rate varied slightly from synthesis to synthesis, it was always sufficiently slow so that the synthesis could be stopped whenever the first exciton peak in the absorption spectrum reached the desired value, which could be easily correlated to the NPs' average size through calibration curves available in the literature ${ }^{28}$. Once the cores had reached the desired size, the reaction could be either stopped by removing the heating mantle to let the solution cool down to RT and the resulting NPs were purified as described below, or 
optionally the temperature could be maintained at $250{ }^{\circ} \mathrm{C}$ and the synthesis was continued further to grow a ZnS shell on the prepared CdSe cores.

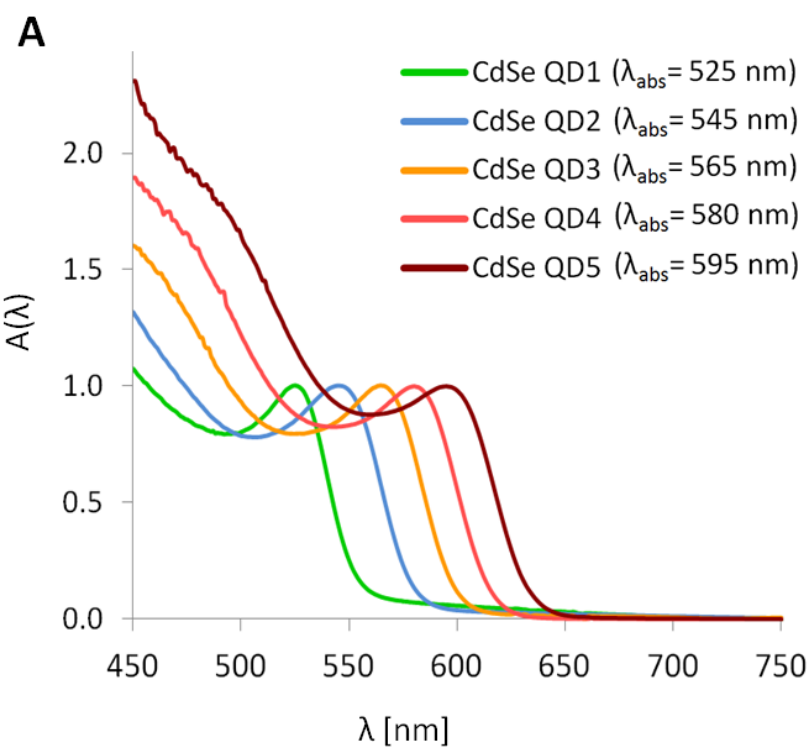

B
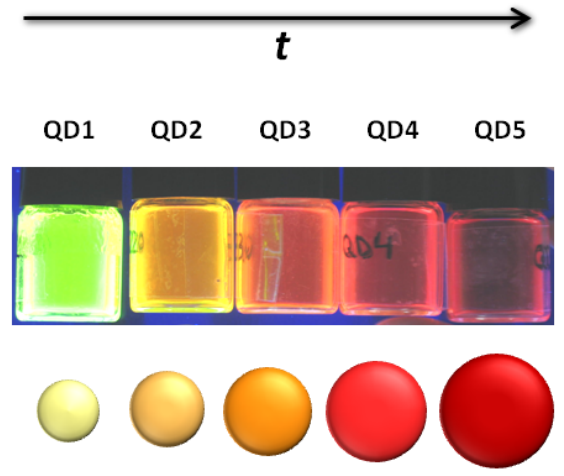

Figure I.7.2. Monitoring the growth of CdSe NPs by A) recording the UV/visible absorption spectra $A(\lambda)$ of aliquot samples that were taken from the reaction flask at different time intervals followed by dilution with chloroform. The peak in the spectrum at wavelength $\lambda_{a b s}$ is due to the excitation of the first exciton ${ }^{29}$. The spectra were normalized to the absorption at the first exciton peak. Upon growth of CdSe NPs, the absorption peak shifts to higher wavelength, as shown here in the five spectra recorded after different time points ( $t=5 \mathrm{~min}, 10 \mathrm{~min}, 15 \mathrm{~min}, 20 \mathrm{~min}, 30 \mathrm{~min}$ ). B) The solutions of the CdSe NPs are fluorescent with tunable excitation wavelength that red upon growth of NPs.

ZnS shell growth: A shell of ZnS can be optionally grown around the CdSe cores, see Figure 1.7.3. Once CdSe NPs have reached the desired size, they can be directly passivated by growing a ZnS shell, without the need of isolating the CdSe NPs from the crude solution ${ }^{30}$. For CdSe cores with a size of $d_{c}=3.82 \mathrm{~nm}$ in diameter, $1.5 \mathrm{~mL}$ of the overcoating stock solution ( $\mathrm{Zn} / \mathrm{S} / \mathrm{TOP}$ solution) was added dropwise to the mixture under vigorous stirring through the addition funnel over a period of $\approx 10 \mathrm{~min}$. It is important that the addition is done dropwise in order to avoid nucleation of ZnS NPs. The $\mathrm{Zn} / \mathrm{S} /$ TOP stock solution was prepared by adding equimolar amounts of the $\mathrm{Zn}$ and $S$ precursors in TOP, that is $1.75 \mathrm{~mL}$ of diethylzinc solution ( $\mathrm{ZnEt}_{2} 1 \mathrm{M}$ in hexane, SigmaAldrich \#296112), and $0.37 \mathrm{~mL}$ of hexamethyldisilathiane ((TMS) ${ }_{2} \mathrm{~S}$, Sigma-Aldrich \#283134) in $10.38 \mathrm{~mL}$ of trioctylphosphine (TOP, Sigma-Aldrich \#718165). Note that the $\mathrm{Zn} / \mathrm{S} / \mathrm{TOP}$ stock solution can be separated and frozen into aliquots to be used in 
next synthesis, being stable once frozen for at least six months. After the addition of $\mathrm{Zn} / \mathrm{S} / \mathrm{TOP}$ solution, the mixture was left to cool down to $90^{\circ} \mathrm{C}$ by removing the heating mantle, and then this temperature was maintained, and the solution was stirred for 3 more hours. Finally, the solution was cooled down to RT by turning the heating off, the NP suspension was transferred to a glass vial and diluted with $10 \mathrm{~mL}$ of anhydrous chloroform.

The amount of added $\mathrm{Zn} / \mathrm{S} / \mathrm{TOP}$ stock solution depends on the CdSe core size and the desired thickness of the $\mathrm{ZnS}$ shell. The amount of of $\mathrm{Zn}$ and $\mathrm{S}$ precursors (i.e. $\mathrm{Zn} / \mathrm{S} / \mathrm{TOP}$ stock solution) needed for growing a ZnS shell of desired thickness can be estimated. First, from the wavelength of the first exciton peak $\lambda_{\text {abs }}$ in the absorption spectrum of the CdSe cores the diameter of the cores $d_{c}$ is estimated via an empirical formula by $\mathrm{Yu}$ et $a l^{28}$, which correlates CdSe NPs core size $\left(d_{c}\right)$ with the corresponding wavelength of the first exciton peak $\left(\lambda_{\mathrm{abs}}\right)$ :

$\mathrm{d}_{\mathrm{c}}=\left(1.6122 \mathrm{~nm} \times 10^{-9} \mathrm{~nm}^{-4}\right) \times \lambda_{\mathrm{abs}}{ }^{4}-\left(2.6575 \times 10^{-6} \mathrm{~nm}^{-3}\right) \times \lambda_{\mathrm{abs}}{ }^{3}+$ $\left(1.6242 \times 10^{-3} \mathrm{~nm}^{-2}\right) \times \lambda_{\mathrm{abs}}{ }^{2}-0.4277 \times \lambda_{\mathrm{abs}}+41.57 \mathrm{~nm} \quad$ (Equation I.7.1)

According to literature, a number of $\mathrm{ZnS}$ monolayers between 1 and 2 is usually the optimum for getting the maximum quantum yield in CdSe/ZnS NPs ${ }^{26,30,31}$. In case of too thin layers the passivation effect is not significant. In case of too thick layers, the ZnS shell may crack due to differences in the lattice constants between CdSe and ZnS, resulting in poor quantum yields. In this work 1.2 monolayers were selected as the desired thickness of ZnS on CdSe NPs. According to the bulk lattice parameters of ZnS, the thickness of one single $\mathrm{ZnS}$ monolayer is $0.31 \mathrm{~nm}$, defined as the distance between consecutive planes along the [002] axis in bulk wurtzite $\mathrm{ZnS}^{26}$. Therefore, the thickness of the $\mathrm{ZnS}$ shell corresponding to 1.2 monolayers is $0.37 \mathrm{~nm}$ (cf. Figure I.1.1).

By knowing the diameter of the CdSe core $\left(d_{c 1}\right)$ from Equation I.7.1 (as the inorganic part comprises here a core and a shell the innermost core is termed $d_{c 1}$ instead of $d_{c}$ ), and the desired thickness of the ZnS shell $\left(d_{c 12}-d_{c 1}\right)=2 \times 0.37 \mathrm{~nm}=0.74 \mathrm{~nm}$, the diameter $d_{c 12}$ of the CdSe/ZnS core/shell NP can be calculated $\left(d_{c 12}=d_{c 1}+0.74 n m\right)$. The volume of the $\mathrm{ZnS}$ shell $\mathrm{V}_{\mathrm{c} 2}$ around one CdSe core thus is the volume of one CdSe NP $\left(V_{c 1}=(\pi / 6) \cdot d_{c 1}{ }^{3}\right)$, substracted from the volume of one CdSe/ZnS NP $\left(V_{c 12}=\right.$ $\left.(\pi / 6) \cdot d_{c 12}{ }^{3}\right), c f$. Equation VIII.1.3.

For example, sample QD4 in Figure I.7.2 corresponds to CdSe NPs with $\lambda_{\text {abs }}=580 \mathrm{~nm}$. According to Equation I.7.1, $d_{c 1}=3.82 \mathrm{~nm}$, and thus $d_{c 12}=3.82 \mathrm{~nm}+0.74 \mathrm{~nm}=4.56$ $\mathrm{nm}$. This leads to volumes $V_{c 1}=29.19 \mathrm{~nm}^{3}$ and $V_{c 2}=V_{c 12}-V_{c 1}=20.46 \mathrm{~nm}^{3}$ of the CdSe 
core and the $\mathrm{ZnS}$ shell, respectively. The ratio of the shell volume to the core volume thus is $\mathrm{V}_{\mathrm{c} 2} / \mathrm{V}_{\mathrm{c1}}=0.70$. As there are $\mathrm{Cd}$ atoms only in the core $\left(\mathrm{N}_{\mathrm{Cd} / \mathrm{NP}}\right.$ per $\left.\mathrm{NP}\right)$, and $\mathrm{Zn}$ atoms only in the shell ( $\mathrm{N}_{\mathrm{Zn} / \mathrm{NP}}$ per NP), the amount of $\mathrm{Cd}$ and $\mathrm{Zn}$ in one NP scales according to Equation VIII.1.9 with the volume of the CdSe core (ratio of $\mathrm{Cd}: \mathrm{Se}=\mathrm{x}: \mathrm{y}=$ 1) and the $Z n S$ shell (ratio of $Z n: S=x: y=1$ ), respectively:

$$
\begin{aligned}
& \mathrm{N}_{\mathrm{Cd} / \mathrm{NP}}=\rho_{\mathrm{CdSe}} \times \mathrm{V}_{\mathrm{c} 1} \frac{\mathrm{N}_{\mathrm{A}}}{\left(\mathrm{M}_{\mathrm{Cd}}+\left(\frac{1}{1}\right) \times \mathrm{M}_{\mathrm{Se}}\right)} \\
& \mathrm{N}_{\mathrm{Zn} / \mathrm{NP}}=\rho_{\mathrm{ZnS}} \times \mathrm{V}_{\mathrm{c} 2} \frac{\mathrm{N}_{\mathrm{A}}}{\left(\mathrm{M}_{\mathrm{Zn}}+\left(\frac{1}{1}\right) \times \mathrm{M}_{\mathrm{S}}\right)}
\end{aligned}
$$

Hereby $M_{C d}=112.4 \mathrm{~g} / \mathrm{mol}, M_{S e}=79.0 \mathrm{~g} / \mathrm{mol}, M_{z n}=65.4 \mathrm{~g} / \mathrm{mol}$, and $M_{S}=32.1 \mathrm{~g} / \mathrm{mol}$ are the molar masses of cadmium, selenium, zinc, and sulfur, respectively. $\rho_{\text {cdse }}=5.82$ $\mathrm{g} / \mathrm{cm}^{3}$ and $\rho_{\mathrm{ZnS}}=4.09 \mathrm{~g} / \mathrm{cm}^{3}$ are the bulk densities of CdSe and $\mathrm{ZnS}$, respectively. Thus the ratio of $\mathrm{Zn}$ to $\mathrm{Cd}$ atoms in one $\mathrm{CdSe} / \mathrm{ZnS} \mathrm{NP}$ is:

$$
\frac{\mathrm{N}_{\mathrm{Zn} / \mathrm{NP}}}{\mathrm{N}_{\mathrm{Cd} / \mathrm{NP}}}=\left(\frac{\mathrm{V}_{\mathrm{c} 2}}{\mathrm{~V}_{\mathrm{c} 1}}\right) \times\left(\frac{\rho_{\mathrm{ZnS}}}{\rho_{\mathrm{CdSe}}}\right) \times\left(\frac{\left(\mathrm{M}_{\mathrm{Cd}}+\mathrm{M}_{\mathrm{Se}}\right)}{\left(\mathrm{M}_{\mathrm{Zn}}+\mathrm{M}_{\mathrm{S}}\right)}\right)=1.38 \times\left(\frac{\mathrm{V}_{\mathrm{c} 2}}{\mathrm{~V}_{\mathrm{c} 1}}\right)
$$

In the case of the sample QD4 in Figure I.7.2 with $\lambda_{\text {abs }}=580 \mathrm{~nm}$ this leads to $\mathrm{N}_{\mathrm{Zn}} / \mathrm{N}_{\mathrm{Cd}}=$ $1.38 \times 0.70 \approx 0.96$. The amount $\mathrm{n}_{\mathrm{Cd} \text { sol }}$ of $\mathrm{Cd}$ added in form of $\mathrm{CdO}$ as precursor during the synthesis of the CdSe cores is known. In the particular case of the synthesis described here it was $\mathrm{n}_{\mathrm{Cd} \text { sol }}=0.234 \mathrm{mmol}$ (mass of added $\mathrm{CdO}=0.03 \mathrm{~g}, \mathrm{M}_{\mathrm{W}}(\mathrm{CdO})=$ $128.4 \mathrm{~g} / \mathrm{mol})$. The amount of $\mathrm{Zn}$ needed in the reaction solution thus is $\mathrm{n}_{\mathrm{Zn} \mathrm{sol}}=\mathrm{n}_{\mathrm{Cd} \mathrm{sol}} \mathrm{X}$ $\left(\mathrm{N}_{\mathrm{Zn}} / \mathrm{N}_{\mathrm{Cd}}\right)=0.234 \mathrm{mmol} \times 0.96 \approx 0.22 \mathrm{mmol}$. Note, that this calculation assumes that all added $\mathrm{Cd}$ is ended up in $\mathrm{CdSe}$ core. The $\mathrm{Zn}$ concentration in the $\mathrm{Zn} / \mathrm{S} / \mathrm{TOP}$ stock solution is $c_{\mathrm{Zn}}=1 \mathrm{MX} 1.75 \mathrm{~mL} /(1.75 \mathrm{~mL}+0.37 \mathrm{~mL}+10.38 \mathrm{~mL})=0.14 \mathrm{M}$. Thus, in order to inject $\mathrm{n}_{\mathrm{Zn} \mathrm{sol}}=0.22 \mathrm{mmol} \mathrm{Zn}$, the volume $\mathrm{V}_{\mathrm{Zn} / \mathrm{S} / \mathrm{TOP} \text { sol }}=\mathrm{n}_{\mathrm{Zn} \mathrm{sol}} / \mathrm{C}_{\mathrm{Zn}}=0.22 \mathrm{mmol} / 0.14 \mathrm{M}$ $\approx 1.5 \mathrm{~mL}$ needs to be dropwise injected.

Note, that this calculation only provides a rough estimate of the amount of $\mathrm{Zn} / \mathrm{S} / \mathrm{TOP}$ stock solution, which is needed for growing the ZnS shell. In fact, during growth of the ZnS shell small aliquots may be taken and the fluorescence analyzed. Shell growth is continued until the quantum yield of the NPs does no longer increase. 

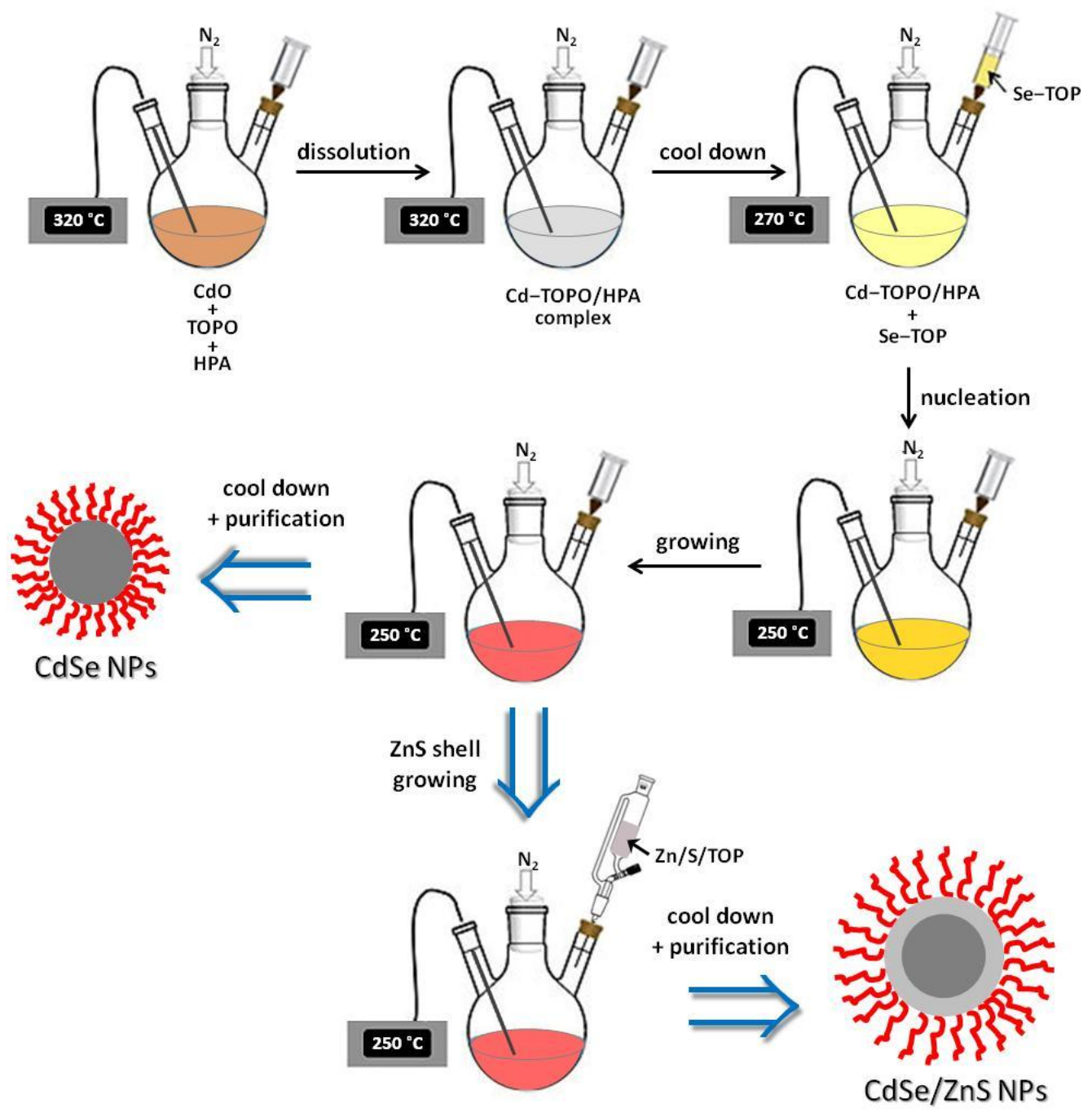

Figure I.7.3. Schematic illustration of the synthesis of CdSe and CdSe/ZnS NPs.

Purification of CdSe or CdSe/ZnS NPs: $10 \mathrm{~mL}$ of anhydrous methanol were added to 10 $\mathrm{mL}$ of the NP suspension, which caused flocculation of the NPs. The NPs were then precipitated by centrifugation (5000 rcf, $5 \mathrm{~min}$ ). Then, the supernatant was discarded and the NP precipitate at the bottom of the vial was resuspended in chloroform. This purification process was repeated two more times (cf. Figure 1.7.4). After the last centrifugation, the supernatant should be colorless and the NPs should be all precipitated on the bottom of the Eppendorf tube in which centrifugation had been carried out. If the supernatant is still colored, the addition of more methanol is needed in order to increase the polarity of the medium, promoting the precipitation of the NPs. Finally, the purified QD NPs (TOPO-capped CdSe or CdSe/ZnS QDs) were dispersed in $10 \mathrm{~mL}$ of anhydrous chloroform and stored in dark. 


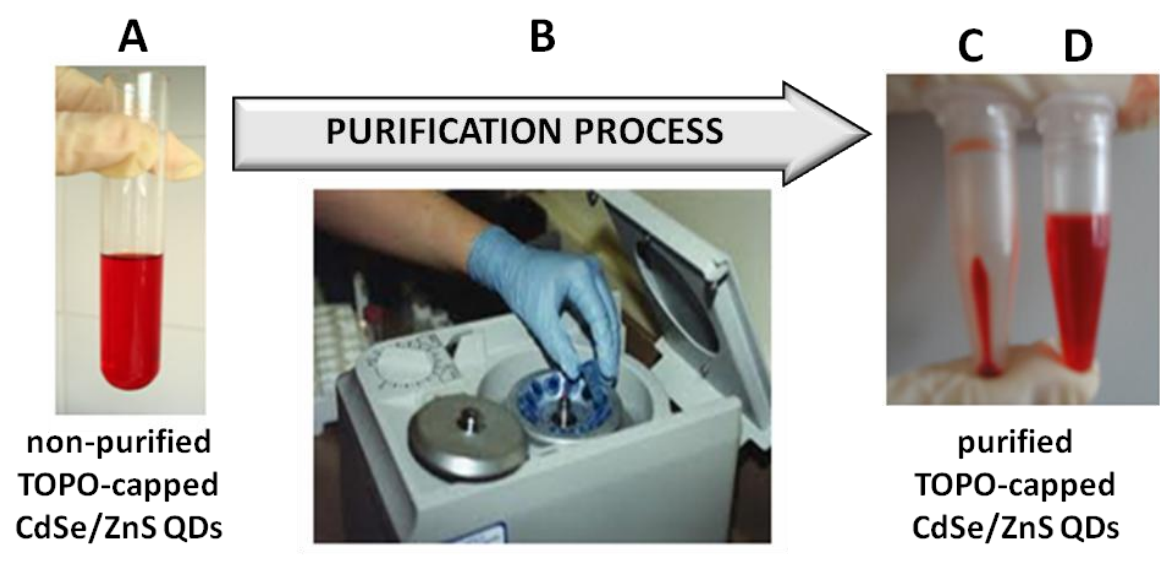

Figure I.7.4. Purification process of the CdSe or CdSe/ZnS QDs by centrifugation and washing with methanol. A) Vial with NPs dispersed in chloroform. B) Centrifugation of the NP suspension after the addition of methanol. C) Precipitated QDs after centrifugation. D) QDs solution after removal of supernatant in $C$ and redispersion in chloroform.

\section{I.8. Synthesis of hydrophobic CdS NPs ( $\approx 2 \mathrm{~nm}$ core diameter)}

Hydrophobic CdS NPs were synthesized by adapting the original method described by W. W. Yu and X. Peng ${ }^{32}$. For the synthesis, a mixture of $4 \mathrm{~g}$ including $12.8 \mathrm{mg}(0.10$ mmol, 2 eq) of cadmium oxide (Sigma, \#202894), $85 \mathrm{mg}(0.30 \mathrm{mmol}, 6 \mathrm{eq})$ of oleic acid (OA, Sigma, \#364525), and $3.90 \mathrm{~g}$ (15.3248 mmol, $306.5 \mathrm{eq})$ of octadecane (ODE, Technical grade $90 \%$, Alfa Aesar, \#L11004) were weighted and mixed in a $50 \mathrm{~mL}$ threeneck round-bottom flask. Then, the mixture was heated to $120{ }^{\circ} \mathrm{C}$ and stirred under nitrogen environment, followed by degassing under vacuum for $5 \mathrm{~min}, c f$. Figure I.8.1.

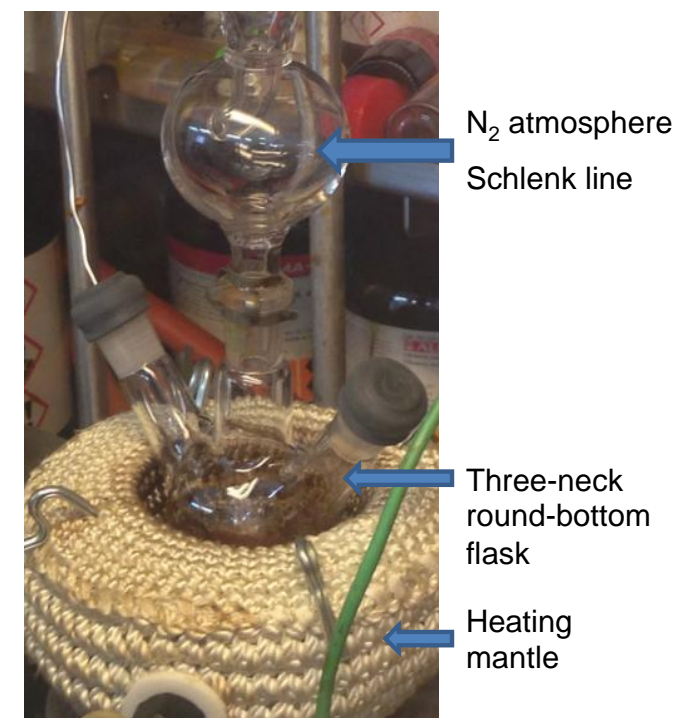


Figure I.8.1. Reaction set-up used for heating the mixture of $C d O$, oleic acid and ODE.

This mixture was then heated to $300{ }^{\circ} \mathrm{C}$, while a solution of sulfur $(0.0016 \mathrm{mg}, 0.05$ $\mathrm{mmol}, 1 \mathrm{eq}$, Sigma, \#414980) in $2 \mathrm{mg}$ of ODE was prepared in a separated glass vial. Since it is very difficult to measure such a small quantity of sulfur, a stock solution was prepared instead by dissolving $8 \mathrm{mg}$ of sulfur in $10 \mathrm{~g}$ of octadecane (ODE, Technical grade 90\%, Alfa Aesar, \#L11004). To efficiently dissolve the sulfur in ODE, the mixture was heated to $85{ }^{\circ} \mathrm{C}$ under stirring.

When the mixture of $\mathrm{CdO}, \mathrm{OA}$, and $\mathrm{ODE}$ reached $300{ }^{\circ} \mathrm{C}$, the $\mathrm{CdO}$ started to decompose, and the initial brownish colored solution started turning into a transparent solution, $c f$. Figure 1.8.2. When the solution became completely transparent, the previously prepared sulfur solution was quickly injected using a syringe, $c f$. Figure I.8.3.

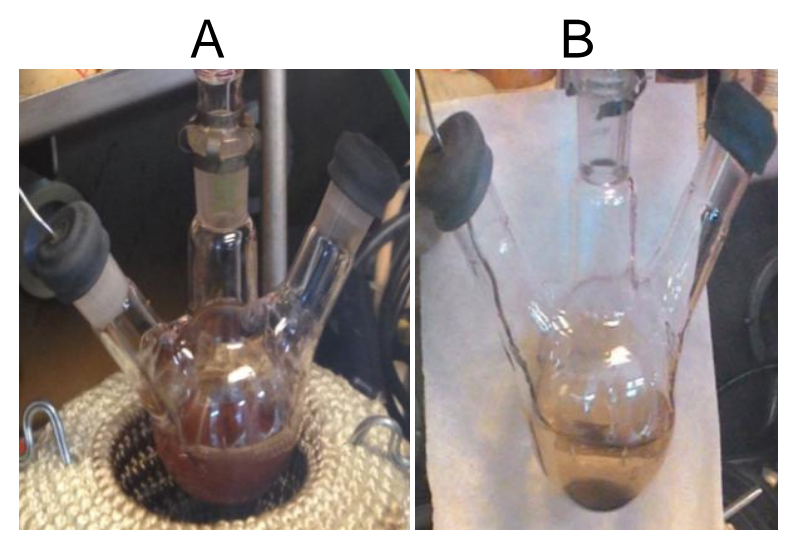

Figure I.8.2. Color change of the mixture before $(A)$ and after $(B)$ decomposition of $C d O$. 


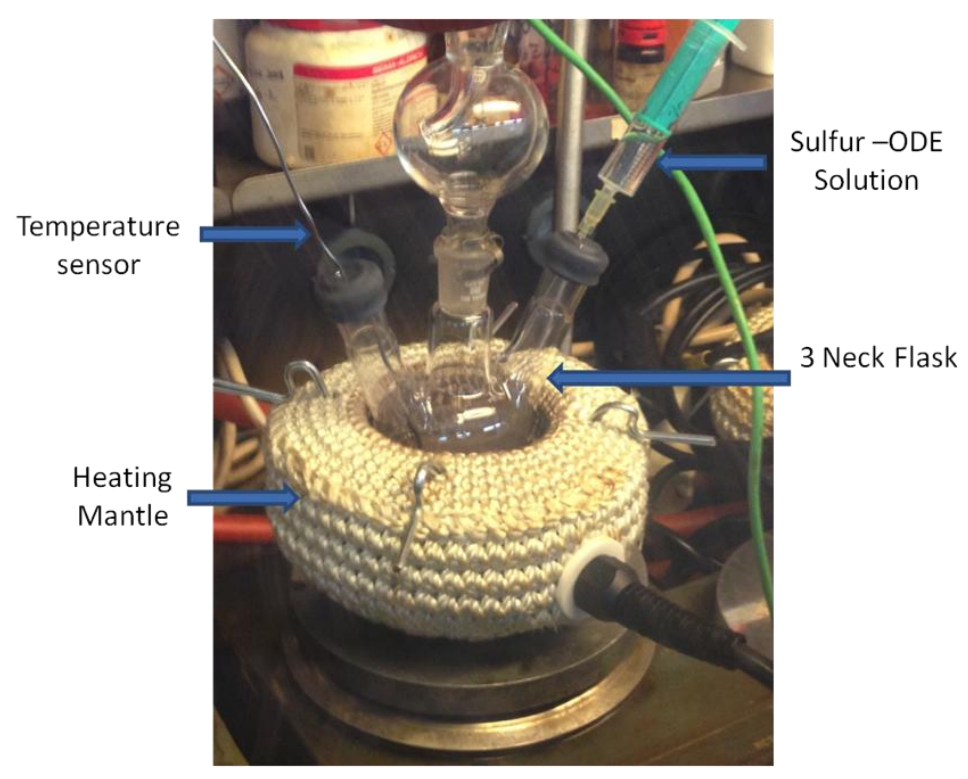

Figure I.8.3. Injection of the sulfur-ODE solution at $300^{\circ} \mathrm{C}$.

After injection of the sulfur-ODE solution, the temperature of the mixture dropped to $240{ }^{\circ} \mathrm{C}$. In case the required size of the QDs was $\mathrm{d}_{\mathrm{c}} \approx 2 \mathrm{~nm}$, the heating mantle was removed just before injecting the sulfur-ODE solution, and the solution was left undisturbed to cool down to RT under the same stirring conditions, $c f$. Figure I.8.5. In case the required size of the QDs was bigger than $2 \mathrm{~nm}$, the temperature of the solution was allowed to recover up to $250{ }^{\circ} \mathrm{C}$ after injection of the sulfur-ODE solution. Depending on the required size of the QDs, the solution was kept for $1-15 \mathrm{~min}$ at 250 ${ }^{\circ} \mathrm{C}$ (i.e. longer times for bigger QDs), cf. Figure I.8.4. The formation of the QDs could be observed by the change of the solution color, from transparent to light yellow. When the desired size (i.e. the envisaged absorption/emission wavelength of the QDs) was achieved, the heating mantle was removed and the solution was allowed to cool down to RT. 


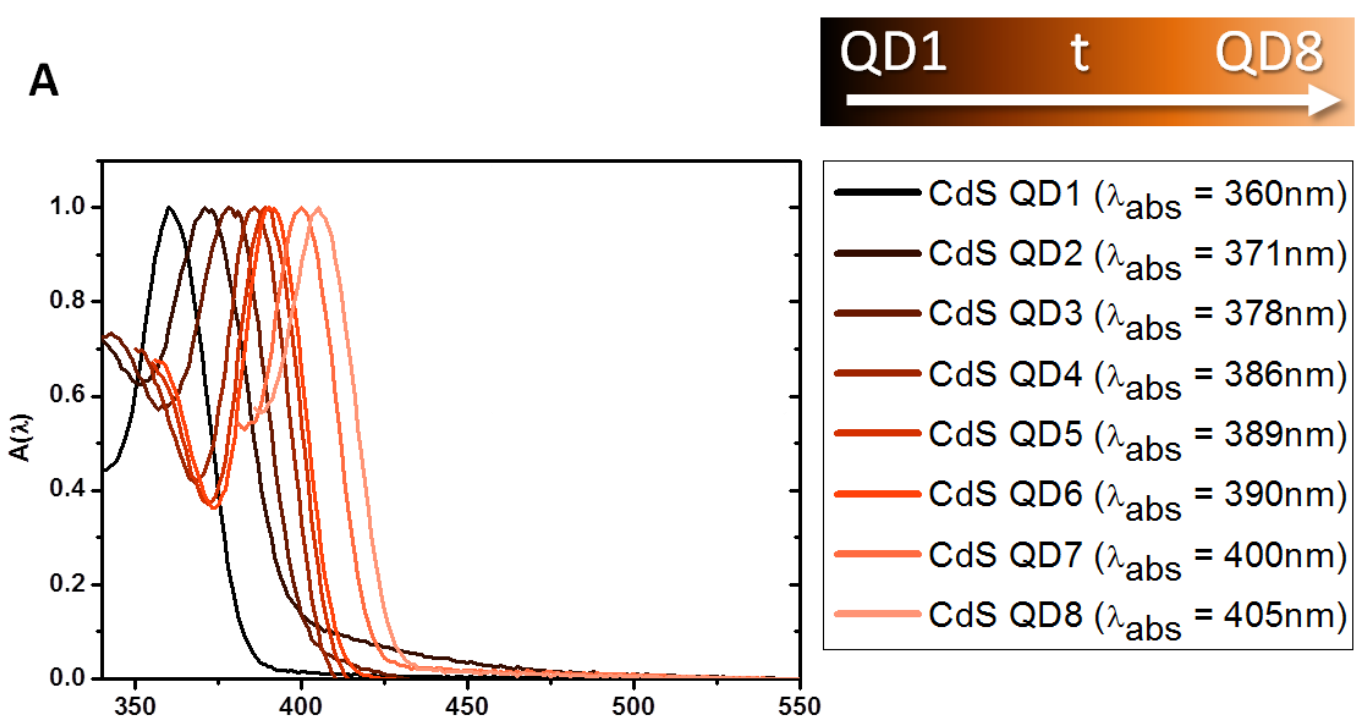

B

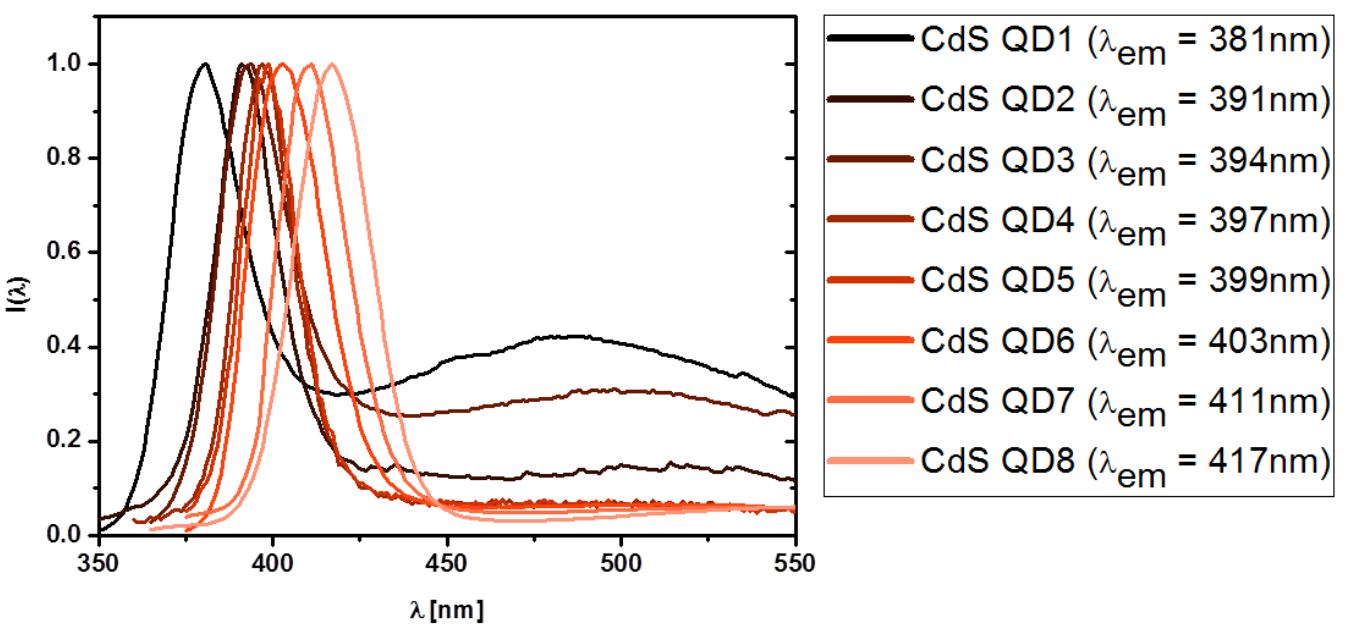

Figure I.8.4. Growth of CdS QDs. Absorption spectra (A) and emission spectra (B) were recorded by taking small aliquots from the reaction solution at different time points (QD1-8). The spectra were normalized to the exciton peak, $A\left(\lambda_{\text {abs }}\right)$ for absorbance and $I\left(\lambda_{\text {em }}\right)$ for emission. Note that upon growth of the QDs, the peak shifts towards higher wavelength.

After cooling down to RT, the solution was transferred into a centrifuge tube. To purify the QDs from ODE, OA, and non-reacted $\mathrm{Cd}$ or $\mathrm{CdO}, 20 \mathrm{~mL}$ acetone was added, and mixed together until the mixture became cloudy. The cloudy mixture was then centrifuged at $2500 \mathrm{rcf}$ for $10 \mathrm{~min}$. The QDs were precipitated and the supernatant was discarded. The precipitated QDs could be redispersed in organic solvent (e.g. chloroform, toluene, etc.). For further cleaning of the QDs, a 1:1 volumetric ratio of chloroform and methanol was used to precipitate the QDs. This process was repeated 1-3 times, until the precipitated QDs were like white powder, in order to ensure the 
purity of the QDs. Finally, the solvent was evaporated and the QDs were dissolved in toluene.
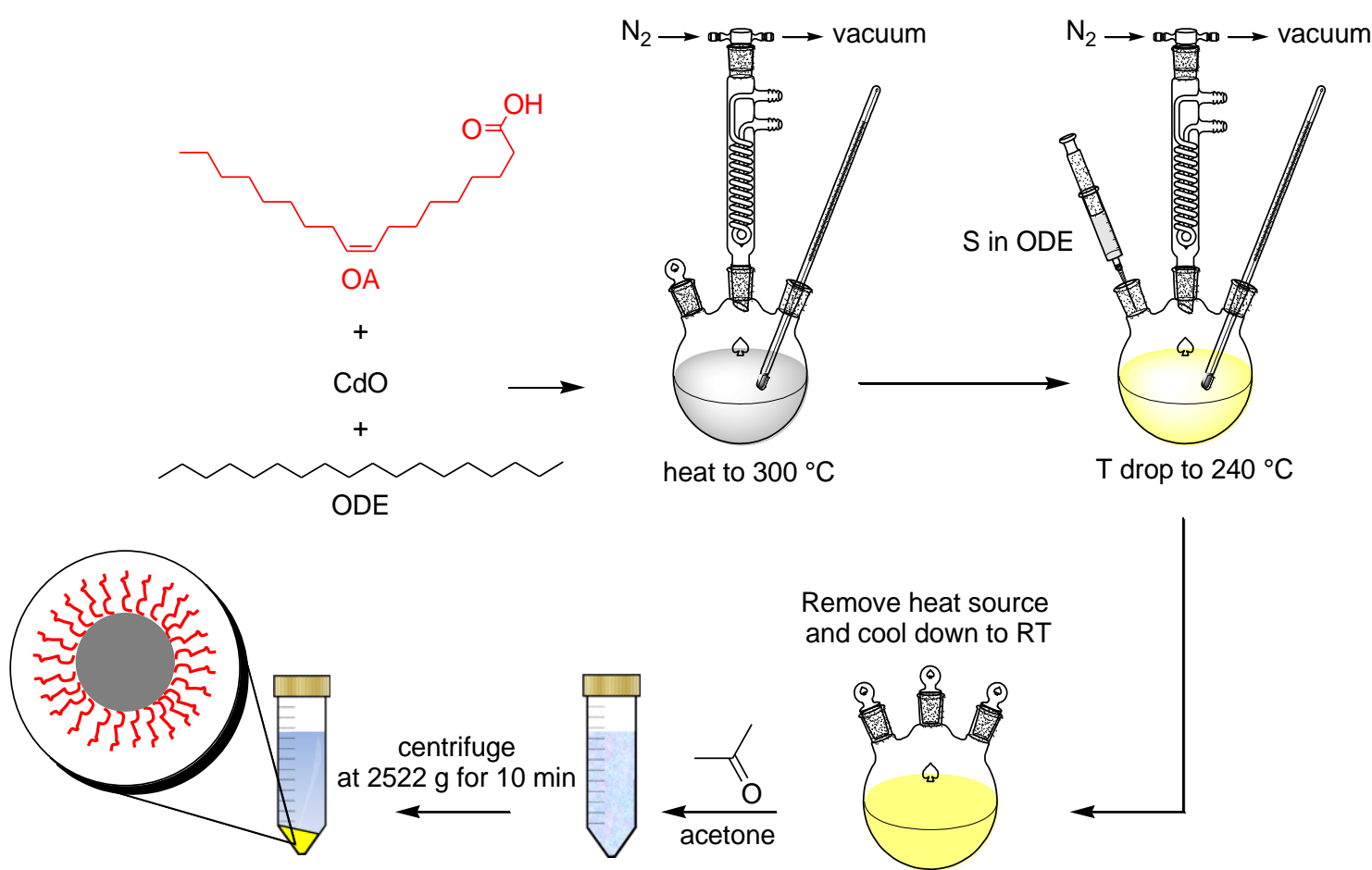

Figure I.8.5. Schematic illustration of the chemical synthesis of hydrophobic CdS NPs $\left(d_{c}\right.$ $\approx 2 \mathrm{~nm}$ ) is shown. $\mathrm{OA}, \mathrm{CdO}$, and ODE were mixed and heated at $300{ }^{\circ} \mathrm{C}$ resulting in a color change from brown to transparent. The sulfur solution in ODE was rapidly injected to induce the formation of NPs as evident from the change from transparent to light yellow (QDs). The QDs were purified (1-3 times) by addition of acetone or methanol followed by precipitation and centrifugation. 


\section{Synthesis of inorganic NPs in aqueous solution leading to hydrophilic NPs}

\section{II.1 General considerations}

II.2 Synthesis of hydrophilic Au NPs ( $\approx 5-100 \mathrm{~nm}$ core diameter)

II.3 Synthesis of hydrophilic Ag NPs ( $\approx 15 \mathrm{~nm}$ core diameter)

II.4 Synthesis of rod-shaped Au NPs (variable size)

II.5 Synthesis of star-shaped Au NPs ( $\approx 70 \mathrm{~nm}$ diameter, distance from tip to tip)

\section{II.1 General considerations}

As detailed in section 1.1, all glassware was first cleaned with detergent followed by aqua regia and carefully rinsed with Milli- $Q$ water and acetone to guarantee extremely cleaned glass surfaces.

In this chapter NPs are prepared in aqueous media, and thus capped by a shell of hydrophilic ligands to maintain their colloidal stability, see Figure II.1.1. In particular, in this chapter the synthesis of different materials such as $\mathrm{Au}$ and $\mathrm{Ag}$ with different shapes is described. In case the NP cores are not of spherical shape, their geometry needs to be described by other parameters than just the core diameter ( $c f$. Figure II.1.1). While this is complex in the form of irregularly shaped NP cores, some other cores can be described best by simple three dimensional objects such as tubes, ellipsoids, cubes, etc. As described in §I.1 the ligand shell adds to the size of the whole NP. Thus in case of an elongated object, apart from the diameter also the effective length is higher than the core lengths, see Equation I.1.1 and Equation II.1.1.

$$
\mathrm{L}_{\mathrm{eff}}=\mathrm{L}_{\mathrm{c}}+2 \times \mathrm{l}_{\text {ligand }}
$$

(Equation II.1.1)

A

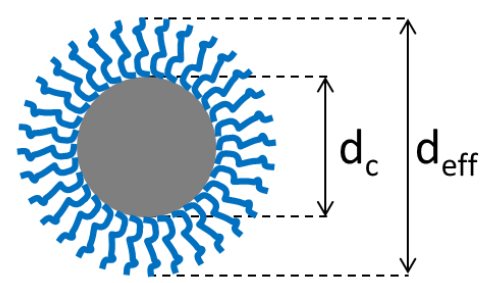

B

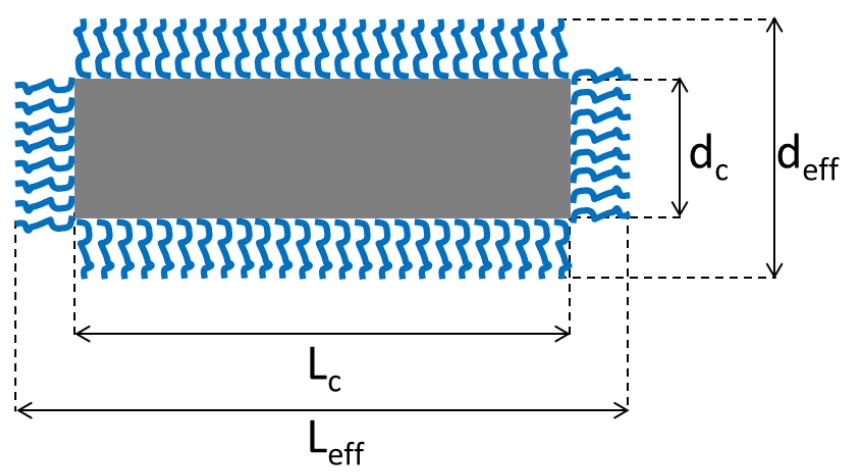


Figure II.1.1. Sketch of A) spherical and B) rod-shaped inorganic NPs, comprising an inorganic core (drawn in gray) and an organic hydrophilic ligand shell (drawn in blue). In the case of nanorods their shape is described by a cylinder with length $L_{c}$ of the inorganic core and $L_{\text {eff }}$ of the NP including the organic ligand shell, and with diameter $d_{c}$ of the inorganic core and $d_{\text {eff }}$ of the whole NP.

\section{II.2 Synthesis of hydrophilic Au NPs ( $\approx 5-100 \mathrm{~nm}$ core diameter)}

Since the first systematic preparation of Au NPs in aqueous media by Turkevich ${ }^{33}$ and then by Frens ${ }^{2}$, significant efforts have been exerted during the last decade to fabricate monodisperse Au NPs with controlled size and shape using wet chemical methods ${ }^{34-38}$. In the following sections we will detail recent synthetic protocols that present improved outcomes compared to the traditional Frens method in several aspects: higher monodispersity and reaction yield of prepared Au NPs, as well as superior tunability and control over NP size and size distribution.

Citrate-capped spherical Au NPs (Cit-Au NPs) can be prepared in aqueous media by reducing gold ions using sodium citrate $(\mathrm{SC})$ as reducing agent and stabilizer. Turkevich et al. and Frens were the first to prepare monodisperse Cit-Au NPs in aqueous solution in a controlled way ${ }^{2,39}$. However, these protocols fail to produce Au NPs with large diameters $\left(d_{c}>50 \mathrm{~nm}\right)$ with acceptable monodispersity. By controlling the reaction conditions (temperature, $\mathrm{pH}$, stirring speed etc.), stoichiometry of the reagents (gold salt to SC ratio), and addition of additives, recent protocols produce different sizes of Cit-Au NPs (up to $200 \mathrm{~nm}$ with excellent monodispersity) as reported by Bastus et $\mathrm{al}^{3}$. Synthetic protocols for spherical Cit-Au NPs with core diameter of $5 \mathrm{~nm}, 13 \mathrm{~nm}, 20 \mathrm{~nm}$, $25 \mathrm{~nm}, 50 \mathrm{~nm}$ and $100 \mathrm{~nm}$ are described below.

Synthesis of Cit-Au NPs ( $5 \mathrm{~nm}$ core diameter): Cit-Au NPs with a core diameter of around $5 \mathrm{~nm}$ were synthesized by following the protocol reported by Piella et al ${ }^{37}$. Briefly, $150 \mathrm{~mL}$ of $2.2 \mathrm{mM}$ sodium citrate (SC, $0.33 \mathrm{mmol}, 13.2 \mathrm{eq}$, Sigma Aldrich, \#W302600), $0.1 \mathrm{~mL}$ of $2.5 \mathrm{mM}$ tannic acid (TA, $0.25 \mu \mathrm{mol}, 0.01 \mathrm{eq}$, Alfa Aesar, \#A17022) (the use of traces of tannic acid helps for the growing of homogeneous seeds; as tannic acid is used at a very small concentration the predominant capping agent is citric acid), and $1 \mathrm{~mL}$ of $150 \mathrm{mM}$ potassium carbonate $\left(\mathrm{K}_{2} \mathrm{CO}_{3}, 0.15 \mathrm{mmol}, 6\right.$ eq, Sigma Aldrich, \#P5833) were placed in a $250 \mathrm{~mL}$ three-necked round-bottomed flask connected with a condenser to prevent the evaporation of the solvent and were heated up with a heating mantle under vigorous stirring. When the temperature reached $70{ }^{\circ} \mathrm{C}, 1 \mathrm{~mL}$ of $25 \mathrm{mM}$ tetrachloroauric acid $\left(\mathrm{HAuCl}_{4}, 25 \mu \mathrm{mol}, 1 \mathrm{eq}\right.$, Strem Chemicals, \#16903-35-8) was quickly injected. The color of the solution changed 
quickly to purple and then to red in $2 \mathrm{~min}$ (see Figure II.2.1). The solution was kept at $70{ }^{\circ} \mathrm{C}$ for $5 \mathrm{~min}$ more, in order to ensure a complete reduction of gold ions.

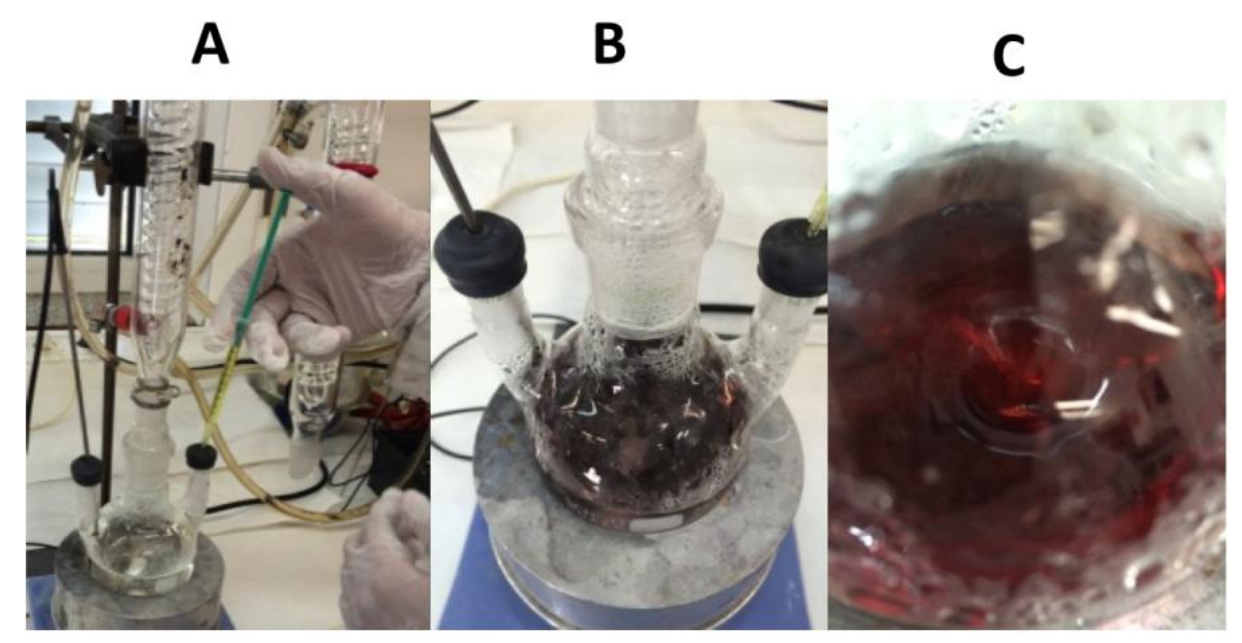

Figure II.2.1. Synthesis of Cit-Au NPs of $\approx 5 \mathrm{~nm}$ in diameter. Photographs showing the reaction flask. A) Before gold injection (note the yellow gold salt solution inside the syringe). B) Immediately after injection of gold salt solution (the solution color changed from pale to purple in less than $10 \mathrm{~s}$ after the gold addition). C) After few minutes (ca. 2 min) after injection of gold salt solution red color indicates the formation of the Cit-Au NPs.

The reduction of a gold salt by sodium citrate in general is described in Equation II.2.1. This equation represents all the following methodologies to obtain spherical Cit-Au NPs. A sketch of the reaction is shown in Figure II.2.2.

$\left.\mathrm{n} \cdot \mathrm{HAuCl}_{4}(\mathrm{aq})+\mathrm{n} \cdot\left(\mathrm{OCOCH}_{2}\right)_{2} \mathrm{C}(\mathrm{OH}) \mathrm{COO}^{-}\right)(\mathrm{aq}) \rightarrow$

$4 n \cdot \mathrm{Cl}^{-}(\mathrm{aq})+4 \mathrm{n} \cdot \mathrm{H}^{+}(\mathrm{aq})+\mathrm{Au}_{\mathrm{n}}^{0}+\mathrm{n} \cdot\left({ }^{-} \mathrm{OCOCH}_{2}\right)_{2} \mathrm{CO}(\mathrm{aq})+\mathrm{n} \cdot \mathrm{CO}_{2}(\uparrow) \quad$ (Equation II.2.1)

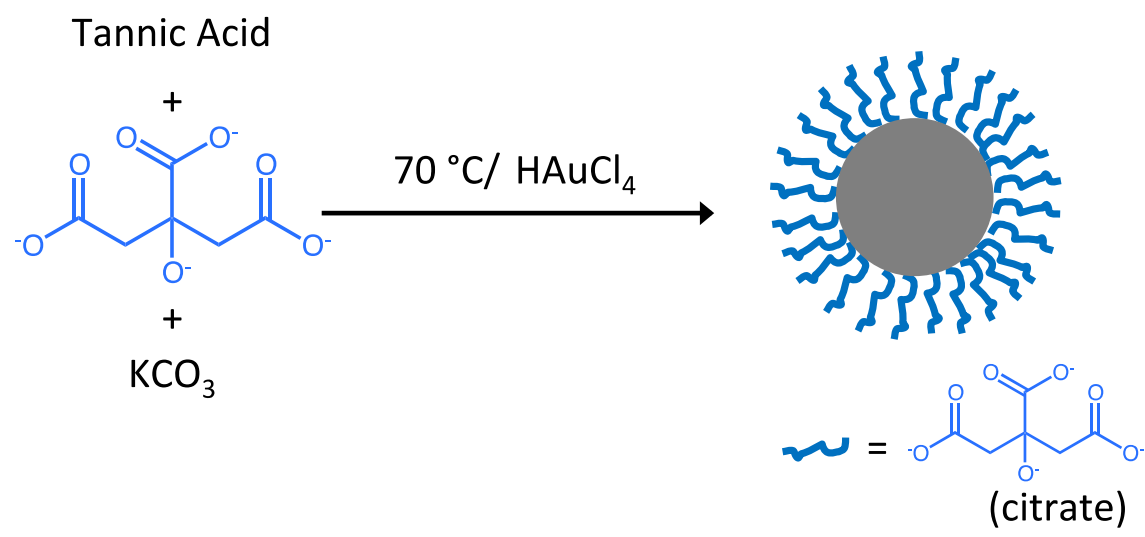

Figure II.2.2. Schematic representation of the synthesis of $5 \mathrm{~nm}$ Cit-Au NPs in water. 
Synthesis of Cit-Au NPs (13 nm core diameter): Cit-Au NPs with a core diameter of $\approx 13 \mathrm{~nm}$ were synthesized by following the protocol reported by Schulz et al ${ }^{36}$. Briefly, $144 \mathrm{~mL}$ of Milli-Q water were placed in a $250 \mathrm{~mL}$ three-necked round-bottomed flask connected with a condenser, and heated up until boiling (ca. $100{ }^{\circ} \mathrm{C}$ ) with a heating mantle. Then, a mixture of sodium citrate $(3.5 \mathrm{~mL}$ of $60 \mathrm{mM}, 0.21 \mathrm{mmol}, 8.4 \mathrm{eq})$ and citric acid (1.5 ml of $60 \mathrm{mM}$, Acros, \#A0350656) was added and kept under vigorous stirring $(450 \mathrm{rpm})$ for $30 \mathrm{~min}$. Two parameters are crucial in this reaction to get a narrow size distribution: the final $\mathrm{pH}$ (which is kept constant by the addition of the buffer citrate/citric), and the heating time (30 min), in which citrate is partially oxidized. To this solution, $0.1 \mathrm{~mL}$ of $30 \mathrm{mM}$ ethylenediaminetetraacetic acid (EDTA, $0.003 \mathrm{mmol}, 0.12 \mathrm{eq}$, Sigma Aldrich, \#EDS) was injected quickly, followed by the addition of $1 \mathrm{~mL}$ of $25 \mathrm{mM}$ hydrogen tetrachloroaurate (III) dissolved in water $(0.025$ $\mathrm{mmol}, 1 \mathrm{eq}$ ). After ca. $60 \mathrm{~s}$ the color of the mixture changed from pale yellow to dull blue and then to wine-red, which is indicative of the formation of Cit-Au NPs (see Figure II.2.3). After that, the heating was switched off (but not the stirring). When the temperature of the mixture had cooled down to $95{ }^{\circ} \mathrm{C}$, the flask with the NP suspension was immersed in ice in order to stop the reaction. It is worth mentioning that this method allows for a fine-tuning to Cit-Au NPs size by employing a seedmediated approach to grow larger NPs. For example, after the synthesis of Au NPs with $13 \mathrm{~nm}$ core diameter as described above, the NP suspension may be cooled down to $95{ }^{\circ} \mathrm{C}$ and upon injection of $1 \mathrm{~mL}$ of $\mathrm{HAuCl}_{4}(25 \mathrm{mM})$ to this seed solution in the same flask bigger NPs may be grown, similar to using $18 \mathrm{~nm}$ seeds, as explained in more detail later in this section.

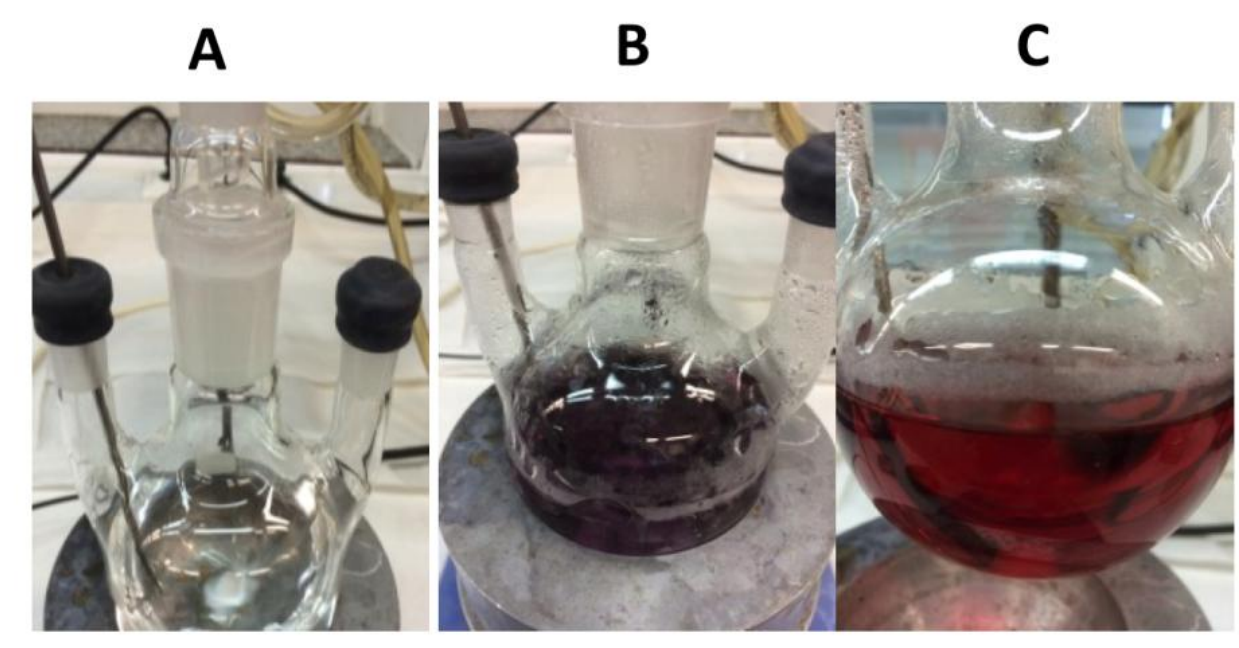

Figure II.2.3. Synthesis of Cit-Au NPs of $\approx 13 \mathrm{~nm}$ in diameter. Photographs showing the reaction flask $A$ ) before gold injection, $B$ ) after injection of gold salt and EDTA solutions the solution color changed to purple/blue, and C) shortly (ca. $1 \mathrm{~min}$ ) after injection, when red color showed up, indicating the formation of Cit-Au NPs. 
Synthesis of Cit-Au NPs (18 nm core diameter): Spherical NPs were synthesized following a modified protocol reported by Bastus et al ${ }^{3}$. Briefly, $150 \mathrm{~mL}$ of a $1.32 \mathrm{mM}$ sodium citrate solution $(0.2 \mathrm{mmol}, 8 \mathrm{eq})$ was added to a $250 \mathrm{~mL}$ three-necked roundbottom flask connected with a condenser, and heated up until boiling with a heating mantle under vigorous stirring. After 5 min of boiling, $1.5 \mathrm{~mL}$ of $25 \mathrm{mM}$ tetrachloroauric acid $\left(\mathrm{HAuCl}_{4}, 0.025 \mathrm{mmol}, 1 \mathrm{eq}\right)$ was injected. A color change was immediately observed from pale yellow to light blue and then to soft red (see Figure II.2.4). The solution was further boiled for $10 \mathrm{~min}$ to allow the NPs to grow and then cooled down with stirring to RT. The resultant Cit-Au NPs had a core diameter of $d_{c}$ $\approx 18 \mathrm{~nm}$.

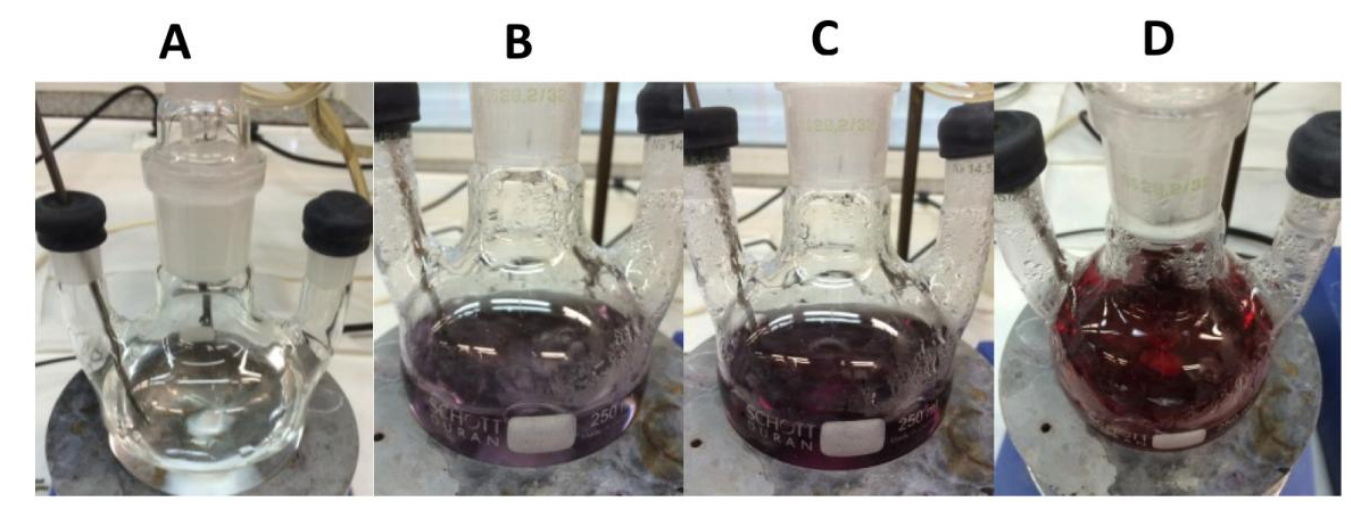

Figure II.2.4. Synthesis of Cit-Au NPs of $\approx 18 \mathrm{~nm}$ in diameter. Real photographs showing the color change at different stages: A) before gold injection; (B) after injection of gold salt solution where the solution color changed to light blue then to purple in (C), and finally to red color indicating the formation of Cit-Au NPs in (D).

Synthesis of Cit-Au NPs $\left(18<\mathrm{d}_{\underline{c}}<100 \mathrm{~nm}\right.$ core diameter): Cit-Au NPs (18 nm core diameter) were used as seeds to prepare larger Cit-Au NPs. After preparing $18 \mathrm{~nm}$ CitAu NPs as described above, the solution was cooled down to $90{ }^{\circ} \mathrm{C}$ and $1 \mathrm{~mL}$ of $25 \mathrm{mM}$ $\mathrm{HAuCl}_{4}(0.025 \mathrm{mmol}, 1 \mathrm{eq}$ ) was injected (see Figure II.2.5). The solution was stirred for $30 \mathrm{~min}$ and this process was repeated twice (total three injections of gold salt per growth step). Importantly, the temperature was set constant to $90{ }^{\circ} \mathrm{C}$ during the whole growing steps. Notice that the temperature is minimally affected by the addition of 1 $\mathrm{mL}$ into the $150 \mathrm{~mL}$ reaction. After that, the sample was diluted by extracting $55 \mathrm{~mL}$ of sample and adding $53 \mathrm{~mL}$ of Milli-Q water and $2 \mathrm{~mL}$ of $60 \mathrm{mM}$ sodium citrate 0.12 $\mathrm{mmol}, 4.8 \mathrm{eq})$, which temporarily drops the temperature and thus, the mixture requires $\mathrm{ca}$. $15 \mathrm{~min}$ to reach the temperature set in the heating mantle (i.e., $90{ }^{\circ} \mathrm{C}$ ). Then, by three consecutive gold additions ( $0.025 \mathrm{mmol}, 1 \mathrm{eq}$, each) separated by 30 min intervals as described above, Cit-Au NPs with $25 \mathrm{~nm}$ were obtained. Likewise, 25 $\mathrm{nm}$ Cit-Au NPs were grown to $50 \mathrm{~nm}$, and later they were grown to $100 \mathrm{~nm}$, by using 
the approach described above (i.e., growing from 18 to $25 \mathrm{~nm}$ Cit-Au NPs, see Figure IX.1.6. for UV/vis absorption spectra of different growing steps). After the synthesis the Cit-Au NPs can be stored for further modification in the presence of free citrate in the solution to ensure colloidal stability of Cit-Au NPs for long time periods (months).

A

B

C

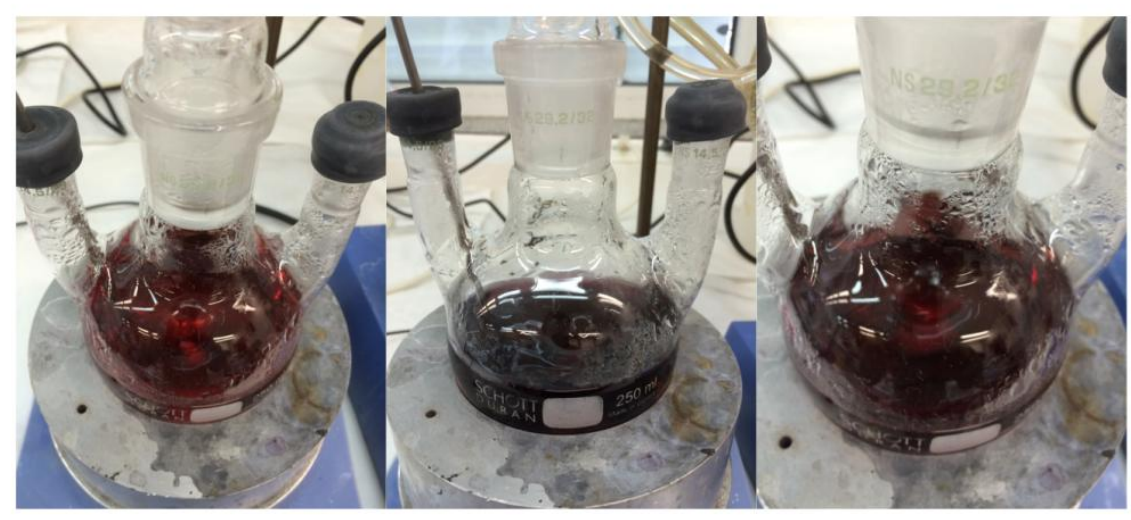

Figure II.2.5. Synthesis of Cit-Au NPs of $\approx 25 \mathrm{~nm}$ in diameter using $18 \mathrm{~nm}$ seeds and the seed-mediated approach. Real photographs showing the color change at different stages: (A) $18 \mathrm{~nm}$ Au NPs seeds; (B) after injection of gold salt solution where the solution color changed to purple in (C) after three injections (30 min reaction time between injections) to prepare Cit-Au NPs of $25 \mathrm{~nm}$ in diameter.

\section{II.3 Synthesis of hydrophilic Ag NPs ( $\approx 15 \mathrm{~nm}$ core diameter)}

Ag NPs can be synthesized with different capping agents in aqueous solution to yield Ag core of different size and shape. ${ }^{40}$ Citric acid has been used as most common capping agent. Synthesizing highly monodisperse citrate-capped silver NPs (Cit-Ag NPs) has been a challenge for long time. There are plenty of synthetic routes developed to synthesize Cit-Ag NPs ${ }^{4,40-43}$. In this section, we briefly describe the synthesis of Cit-Ag NPs (15 nm in diameter) as developed by Bastus et al. ${ }^{4}$. The described procedure employs tannic acid (TA) (basically its phenolic hydroxyl groups) as well as citric acid as reducing agents. The use of a small amount of tannic acid controls the size during the growth process, yielding unprecedented narrow size distributions. TA acts as both a reducing agent and a size control agent. The advantage of this method is that by varying the concentration of TA, a wide size range of highly monodisperse Cit-Ag NPs can be synthesized. 
<smiles>O=C1C=C(C(=O)COC(=O)c2cc(C(=O)O)cc(O)c2O)C=C(O)C1=O</smiles>

Phenol
Quinone
(Equation II.3.1)

(Equation II.3.2)

$$
\mathrm{Ag}^{+}+\mathrm{e}^{-} \rightarrow \mathrm{Ag}^{0}
$$

To synthesize $15 \mathrm{~nm}$ Cit-Ag NPs, $100 \mathrm{~mL}$ aqueous solution of sodium citrate (SC) and tannic acid (TA) were taken in a three-neck round bottom flask such that the final concentration of SC and TA are $5 \mathrm{mM}$ and $0.1 \mathrm{mM}$, respectively. At this stage the solution looks colorless, as it can be seen in the photographs given in Figure II.2.1A. The flask was heated with a heating mantle (with a magnetic stirrer) and a condenser was used to avoid the evaporation of solvent. When the solution started boiling, $1 \mathrm{~mL}$ of an aqueous solution of $25 \mathrm{mM} \mathrm{AgNO}_{3}$ was rapidly injected into the solution. The color of the solution immediately turned to bright yellow (cf. Figure II.2.1B), confirming the formation of NPs. After cooling down the solution, it was centrifuged at $5000 \mathrm{rcf}$ for $10 \mathrm{~min}$ in order to precipitate the NPs. Then, the supernatant was removed and the NP precipitate was redispersed in $0.25 \mathrm{mM}$ sodium citrate solution to get the purified Cit-Ag NPs.
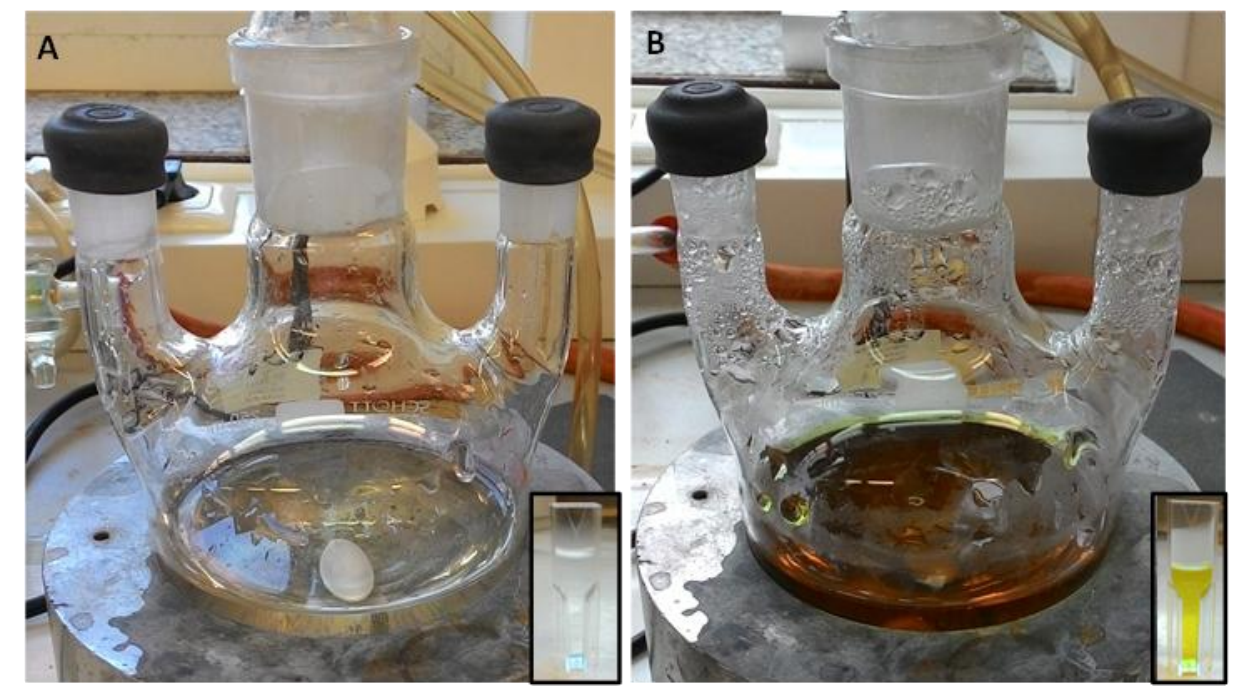

Figure II.2.1. Synthesis of Cit-Ag NPs of $\approx 15 \mathrm{~nm}$ in core diameter. Photographs showing the solution color $(A)$ before and $(B)$ after injection of silver nitrate solution. Yellow solution color in B indicates the formation of Cit-Ag NPs. For more clarity, a photograph of a cuvette containing the solution is given in the inset 


\section{II.4 Synthesis of rod-shaped Au NPs (variable size)}

Rod-shaped Au NPs (gold nanorods, Au NRs) with the longitudinal surface plasmon resonance (SPR) located at $\lambda_{\text {SPR }}=650,800,830$ and $1050 \mathrm{~nm}$ were prepared by a seedmediated method following previously published protocols ${ }^{34,44}$.

The wet-synthesis method to prepare Au NRs was pioneered by the groups of Murphy ${ }^{45}$ and El Sayed ${ }^{46}$ in the early 2000s. In general, a solution of small gold seeds (NPs of $\mathrm{d}_{\mathrm{c}} \approx 3-4 \mathrm{~nm}$ in diameter) is synthetized in the presence of the cationic surfactant hexadecyltrimethylammonium bromide (CTAB), and silver ions. These seeds are further grown in a growth solution that contains more gold ions, silver ions, and CTAB. The gold ions in the growth solution are partially reduced to $\mathrm{Au}(\mathrm{I})$ to allow complete reduction to $A u(0)$ only at the surface of the added seeds, in order to promote growth of $\mathrm{Au}$ NRs and to prevent undesired nucleation and formation of nanospheres as side product. The presence of silver ions improves the shape yield and controls the final aspect ratio (length to width ratio) of the nanorods. CTAB is the shape-directing agent that promotes the unidirectional growth and acts simultaneously as a capping agent to form CTAB-capped gold nanorods (CTAB-Au NRs) ${ }^{47}$. The shape and size of the prepared $\mathrm{Au}$ nanorods can be tuned primarily by varying silver nitrate levels, but generally by controlling the reaction conditions and the level of used chemicals (cf. Table II.4.1).

The traditional protocols to prepare CTAB-Au NRs indicate the use of high concentration of CTAB (0.1 M), which is a cytotoxic and thus limits the use of CTAB-Au NRs in biomedical applications ${ }^{48}$. Significant effort has been done to prepare CTAB-Au NRs in low-CTAB growth solution. In 2012, Murray's group proved that the presence of aromatic additives in the growth solution reduces the required amount of CTAB to the half (this procedure will be termed Protocol 1 below) ${ }^{34}$. In 2013, the same group published a modified method in which the CTAB concentration was reduced to 0.037 $M$. This methodology employs a binary surfactant mixture: CTAB and sodium oleate ( $\mathrm{NaOL}$ ) (this procedure will be termed Protocol 2 below) ${ }^{44}$.

Synthesis of the seed solution: A seed solution was prepared by mixing $5 \mathrm{~mL}$ of $0.5 \mathrm{mM}$ $\mathrm{HAuCl}_{4}(0.5 \mathrm{mmol}, 1 \mathrm{eq}$ ) with $5 \mathrm{~mL}$ of $0.2 \mathrm{M} \mathrm{CTAB}$ (200 mmol, $396 \mathrm{eq}$, Sigma Aldrich, $\#$ H9151) in a $40 \mathrm{~mL}$ tube. To this solution, $0.6 \mathrm{~mL}$ of $0.01 \mathrm{M}$ freshly prepared $\mathrm{NaBH}_{4}(10$ mmol, $19.8 \mathrm{eq}$, Sigma Aldrich \#71321) was added under stirring. The solution color changed immediately to brownish-yellow (cf. Figure II.4.1). The seed solution, containing $\sim 4 \mathrm{~nm}$ core diameter Au NPs capped with CTAB, was vigorously stirred at $1200 \mathrm{rpm}$ for $2 \mathrm{~min}$ and kept at $25^{\circ} \mathrm{C}$. 
Protocol 1: Synthesis of CTAB capped gold nanorods (CTAB-Au NRs) in the presence of 5-bromosalicylic acid. This protocol ${ }^{34}$ was used to prepare CTAB-Au NRs with longitudinal surface plasmon resonance at $\lambda_{S P R}=650 \mathrm{~nm}$ and $825 \mathrm{~nm}$. For this purpose, $9.0 \mathrm{~g}$ of CTAB $(24.6 \mathrm{mmol}, 98.5 \mathrm{eq})$ and $0.8 \mathrm{~g}$ of sodium salicylate $(5 \mathrm{mmol}, 20 \mathrm{eq}$, Sigma, \#S3007) in case of $\lambda_{S P R}=650 \mathrm{~nm}$ or $1.1 \mathrm{~g}$ of 5-bromosalicylic acid ( $5 \mathrm{mmol}, 20$ eq, Sigma, \#461814) in case of $\lambda_{S P R}=825 \mathrm{~nm}$ were dissolved in $250 \mathrm{~mL}$ of warm water $\left(60{ }^{\circ} \mathrm{C}\right)$ in a $500 \mathrm{~mL}$ Erlenmeyer flask. The solution was allowed to cool to $30{ }^{\circ} \mathrm{C}$ and then silver nitrate dissolved in water $\left(\mathrm{AgNO}_{3}, 4 \mathrm{mM}\right)$ was added as per Table II.4.1. The mixture was kept undisturbed at $30{ }^{\circ} \mathrm{C}$ for $15 \mathrm{~min}$, after which $250 \mathrm{~mL}$ of aqueous 1 $\mathrm{mM} \mathrm{HAuCl}_{4}$ solution $(0.25 \mathrm{mmol}, 1 \mathrm{eq})$ was added. After $15 \mathrm{~min}$ of slow stirring (400 rpm), ascorbic acid solution was added (64 mM) as per Table II.4.1. The solution was stirred for $30 \mathrm{~s}$, until it became colorless. The color change is due to the incomplete reduction of $\mathrm{Au}(\mathrm{III})$ to $\mathrm{Au}(\mathrm{I})$ by ascorbic acid. Finally, seed solution (freshly prepared as described above) was injected into the growth solution as per Table II.4.1. The solution was mixed gently and left undisturbed overnight, to allow for a compete growth of CTAB-Au NRs. CTAB-Au NRs were collected by centrifugation and the supernatant was discarded. The centrifuged CTAB-Au NRs pellets were finally redispersed in water.

\begin{tabular}{|c|c|c|c|c|c|}
\hline$\lambda_{\text {SPR }}[\mathbf{n m}]$ & $\mathbf{V}_{\mathrm{HCl}_{-} \text {sol }}[\mathrm{mL}]$ & $\mathbf{p H}$ & $\mathbf{V}_{\text {AgNO3_sol }_{\text {sol }}[\mathrm{mL}]}$ & $\mathbf{V}_{\text {AA_sol }_{-}[\mathrm{mL}]}$ & $\mathbf{V}_{\mathrm{NP}_{-} \text {sol }}[\mathrm{mL}]$ \\
\hline 650 & 0 & 3.05 & 6 & 1 & 0.8 \\
\hline 825 & 0 & 2.00 & 18 & 2 & 0.4 \\
\hline
\end{tabular}

Table II.4.1. Synthesis of CTAB-Au NRs in the presence of 5-bromosalicylic acid, with different SPR wavelength $\lambda_{S P R}$ as detailed in the text. $V_{H C I}$ sol $r e f e r s$ to the volume of $\mathrm{HCl}$, which was used to optimize the $\mathrm{pH}$ of the growth solution. $V_{\text {AgNO3_sol }}$ refers to the volume of added $\mathrm{AgNO}_{3}$ solution. $\mathrm{V}_{\mathrm{AA} \text { sol }}$ refers to the volume of added ascorbic acid solution. $V_{N P_{-} s o l}$ refers to the volume of added NP seed solution.

Synthesis of CTAB capped gold nanorods (CTAB-Au NRs) in the presence of sodium oleate (Protocol 2): This protocol ${ }^{44}$ was used to prepare CTAB-Au NRs with longitudinal surface plasmon resonance at $\lambda_{S P R}=790 \mathrm{~nm}$ and $1050 \mathrm{~nm}$. For this purpose, $7.0 \mathrm{~g}$ of CTAB (19.1 mmol, $76.6 \mathrm{eq})$ and $1.234 \mathrm{~g}$ of sodium oleate ( $\mathrm{NaOL}, 4$ $\mathrm{mmol}, 16.2 \mathrm{eq}$, Sigma Aldrich, \#07501) were dissolved in $250 \mathrm{~mL}$ of Milli-Q water at 50 ${ }^{\circ} \mathrm{C}$. After dissolving the reactants, the solution was cooled down to $30{ }^{\circ} \mathrm{C}$ and then, $\mathrm{a}$ specific amount of $\mathrm{AgNO}_{3}$ (4 mM) was added as per Table II.4.2 and the solution was kept at $30{ }^{\circ} \mathrm{C}$ for $15 \mathrm{~min}$. To this solution, $250 \mathrm{~mL}$ of $1 \mathrm{mM} \mathrm{HAuCl}_{4}(0.25 \mathrm{mmol}, 1 \mathrm{eq})$ were added and the solution color changed from dark yellow to colorless during 90 min of stirring at $700 \mathrm{rpm}$. To control the aspect ratio of Au NRs, $\mathrm{HCl}$ (37 wt\% in water, 
12.1 M, Sigma Aldrich, \#320331) was added to adjust the $\mathrm{pH}$ as per Table II.4.2. The solution was stirred at $400 \mathrm{rpm}$ for $15 \mathrm{~min}$. After this, $1.25 \mathrm{~mL}$ of $0.064 \mathrm{M}$, ascorbic acid $(0.08 \mathrm{mmol}, 0.32 \mathrm{eq})$ was added and the solution was vigorously stirred for $30 \mathrm{~s}$. Finally, a small amount of seed solution was injected into the growth solution as per Table II.4.1). The solution was stirred for $30 \mathrm{~s}$, and kept at $30{ }^{\circ} \mathrm{C}$, without stirring overnight to allow a compete growth of CTAB-Au NRs. Finally the CTAB-Au NRs were collected by centrifugation and the supernatant was discarded. The centrifuged CTABAu NRs pellets were redispersed in water.

\begin{tabular}{|c|c|c|c|c|c|}
\hline$\lambda_{\text {SPR }}[\mathrm{nm}]$ & $\mathbf{V}_{\mathrm{HCl}_{-} \text {sol }}[\mathrm{mL}]$ & $\mathbf{p H}$ & $\mathbf{V}_{\text {AgNO3_sol }[\mathrm{mL}]}$ & $\mathbf{V}_{\mathrm{AA}_{-} \text {sol }}[\mathrm{mL}]$ & $\mathbf{V}_{\mathrm{NP}_{\text {_sol }}[\mathrm{mL}]}$ \\
\hline 790 & 2.4 & 1.68 & 18 & 1.25 & 0.4 \\
\hline 1050 & 5 & 1.2 & 24 & 1.25 & 0.8 \\
\hline
\end{tabular}

Table II.4.2. Synthesis of CTAB-Au NRs in the presence of sodium oleate, with different SPR wavelength $\lambda_{S P R}$ using protocol 2 as detailed in the text. $V_{H C I}$ sol $r e f e r s$ to the volume of $\mathrm{HCl}$, which was used to optimize the $\mathrm{pH}$ of the growth solution. $V_{\mathrm{AgNO}_{\text {_sol }}}$ refers to the volume of added $\mathrm{AgNO}_{3}$ solution. $V_{A A_{-} \text {sol }}$ refers to the volume of added ascorbic acid solution. $V_{N P}$ sol $r e f e r s$ to the volume of added seed NP solution. 

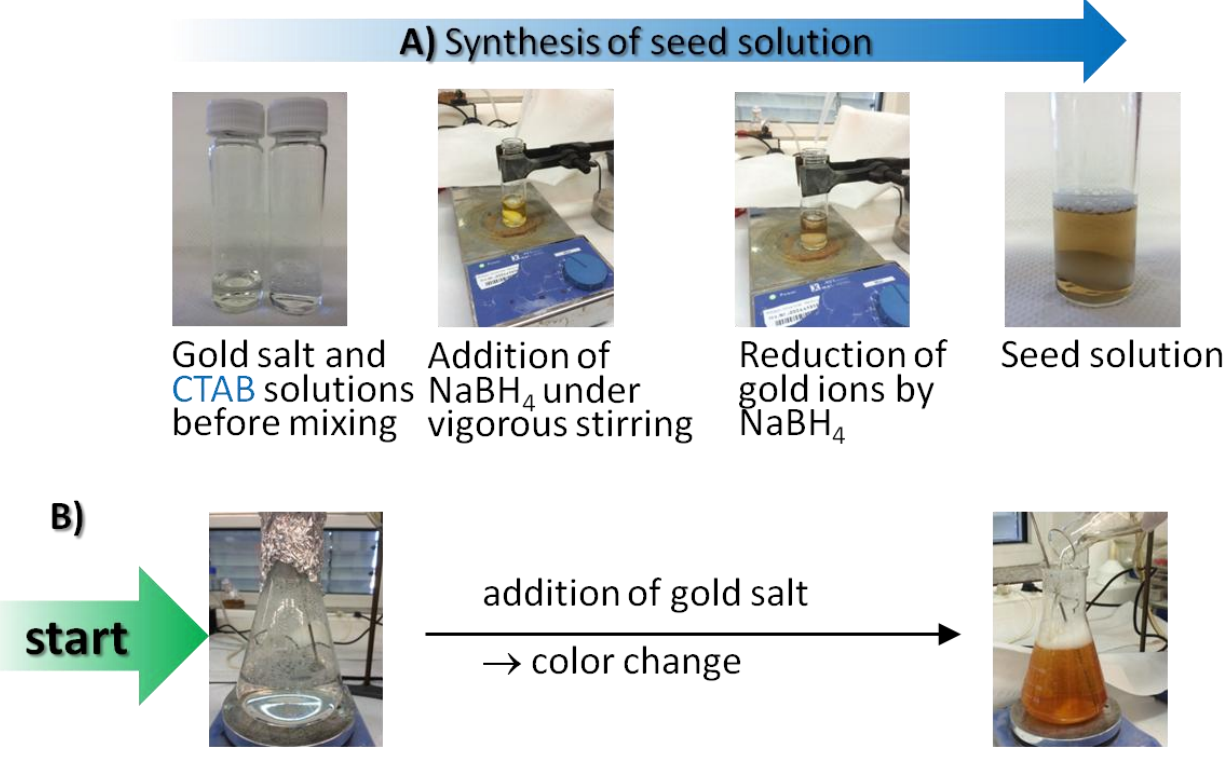

CTAB +
sodium salicylate

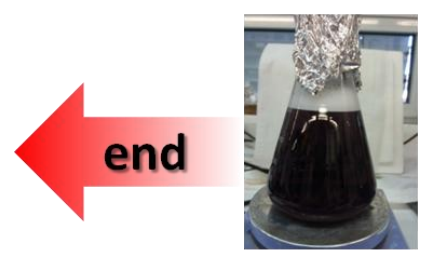

injection of seed solution

$\rightarrow$ Au NPs formation after 5 min

complete Au NPs formation

after $12 \mathrm{~h}$ reaction time

1) 5 min reaction time

2) Injection of $A A$

3) 30 sec reaction time

$\downarrow$

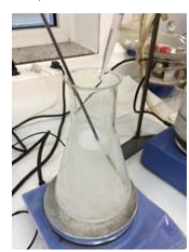

Figure II.4.1. Photographs of steps involved in the preparation of CTAB-Au NRs, using wet chemical seed-mediated method (in the presence of 5-bromosalicylic acid). A) Preparation of CTAB-capped seeds. B) Growth of CTAB-Au NRs.

\section{II.5 Synthesis of star-shaped Au NPs ( $\approx 70 \mathrm{~nm}$ diameter, distance from tip to tip)}

Gold nanostars can be prepared following an unpublished modification of the protocol reported by Vo-Dihn ${ }^{49}$. Typically, gold nanostars are grown using spherical gold NPs as seeds, and using $\mathrm{Ag}^{+}$ions as blocking facet agents. These $\mathrm{Ag}^{+}$ions promote the tip growth on the surface of the spherical NPs. The reduction of the gold salt at RT using ascorbic acid promotes the seed growth instead of independent nucleation ${ }^{20}$.

Briefly, in a clean glass vial, $20 \mathrm{~mL}$ of an aqueous solution of $0.25 \mathrm{mM} \mathrm{HAuCl}_{4}(5 \mu \mathrm{mol}$, 1 eq) was mixed with $0.03 \mathrm{~mL}$ of $1 \mathrm{M} \mathrm{HCl}(0.03 \mathrm{mmol}, 6 \mathrm{eq})$ and $1.5 \mathrm{~mL}$ of Au NPs of 13 $\mathrm{nm}$ diameter, as prepared according to the protocol given in §II.2. To this mixture, 0.15 $\mathrm{mL}$ of $1 \mathrm{mM} \mathrm{AgNO}_{3}(0.15 \mu \mathrm{mol}, 0.03 \mathrm{eq}$ ) and $0.15 \mathrm{~mL}$ of $66.67 \mathrm{mM}$ ascorbic acid (10 $\mu \mathrm{mol}, 2 \mathrm{eq}$ ) were added simultaneously. After $1 \mathrm{~min}, 1 \mathrm{~mL}$ of an aqueous solution of 10 
$\mathrm{mg} / \mathrm{mL} \alpha$-metoxy- $\omega$-thiol-poly-(ethylene glycol (mPEG-SH; molecular weight $\mathrm{M}_{\mathrm{w}}=750$ $\mathrm{Da}$; $13.3 \mathrm{\mu mol}, 2.67 \mathrm{eq}$, Rapp polymer, \#12750-40) and $0.05 \mathrm{~mL}$ of $2 \mathrm{M}$ sodium hydroxide ( $\mathrm{NaOH}, 0.1 \mathrm{mmol}, 20 \mathrm{eq}$, Carl Roth \#6771.3) were added simultaneously (cf. Figure II.5.1). After that, the solution was immersed in ice in order to stop the reaction. The NPs can be stored as prepared, or purified by centrifugation (15 min, 4000 rcf).

A)

\section{Color change upon reaction time $(\mathrm{t})$}

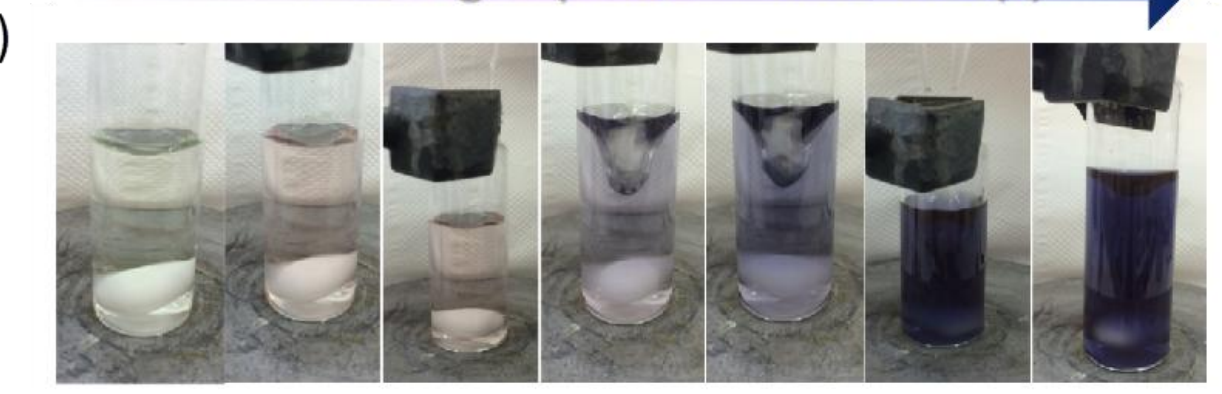

B)

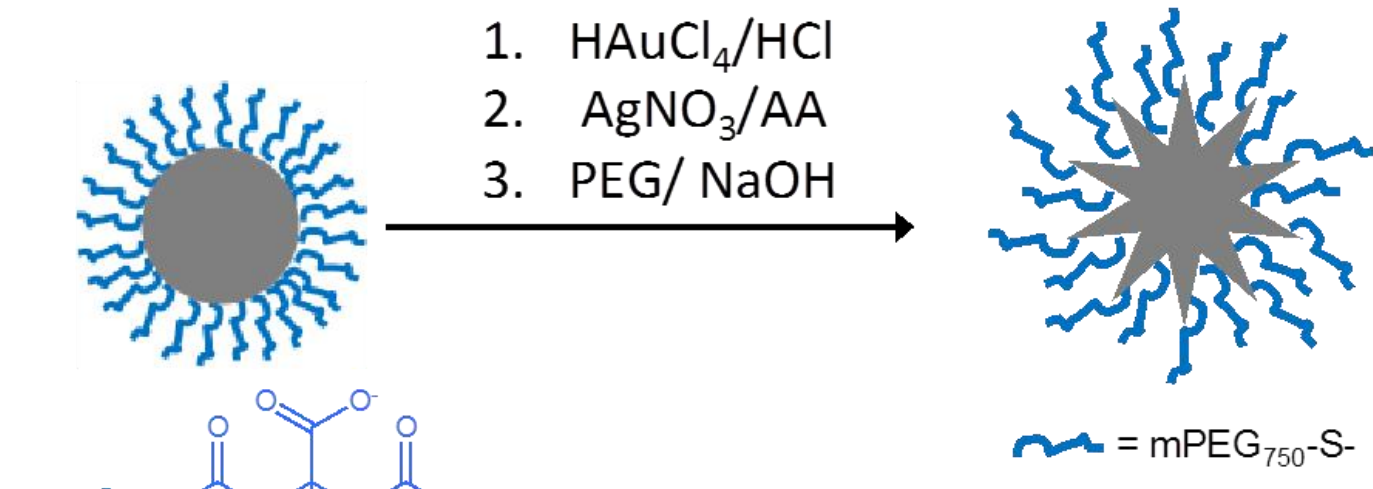

Figure II.5.1. A) Synthesis process of PEG-coated star-shaped Au NPs. After addition of $\mathrm{AgNO}_{3}$ and $\mathrm{AA}$, the solution color changed from soft red to light blue in a few seconds, and then to dark blue, which is indicating the formation of gold nanostars. B) The cartoon demonstrates the seed mediated growth of spherical gold NPs (seeds) into star-shaped Au NPs. The final surface functionalization of the resulting NPs is PEG-SH, as mediated by displacement of physically adsorbed citrate ions by chemically assembled PEG-S molecules via the strong S-Au bond. 
III. Ligand exchange procedures for hydrophilic NPs maintaining their hydrophilic character

\section{III.1 General considerations}

III.2 Ligand exchange on citrate-capped Au NPs with bis(p-sulfonatophenyl)phenylphosphine

III.3 Ligand exchange on citrate-capped Au NPs with thiolated polyethyleneglycol (PEG)

\section{III.1 General considerations}

Often the original hydrophilic ligands used for the synthesis of NPs in aqueous solutions are not adequate to maintain excellent colloidal stability and/or not the ligand of choice for a desired application. The original ligands can be replaced in a ligand exchange procedure based on a superior affinity of the displacing ligand and/or concentration difference ${ }^{50}$. The general scheme of the ligand exchange process is depicted in Figure III.1.1. Despite the availability of various examples in the literature, this section will describe few selected examples.

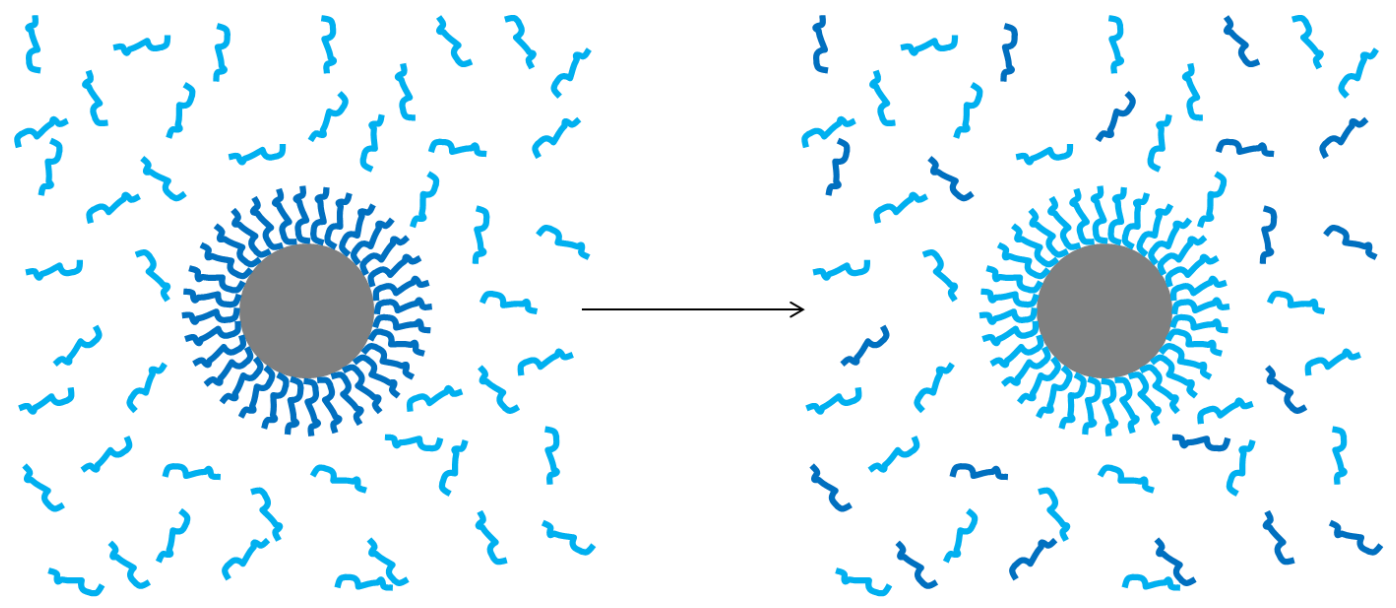

Figure III.1.1. Ligand exchange reaction at the surface of Au NPs. After synthesis, the depicted Au NP core is capped by hydrophilic ligands (drawn in dark blue). In the case Au NPs are incubated with new ligands in excess (drawn in light blue), the new ligands can displace the original ones.

\section{III.2 Ligand exchange on citrate-capped Au NPs with bis(p-sulfonatophenyl)- phenylphosphine}


The citrate ions are physically adsorbed on the surface of citrate-caped Au NPs, i.e. the ligands are only weakly associated to the Au NPs' surface. For example, citrate ligands tend to desorb from the surface of Cit-Au NPs upon dialysis, dilution, or repeated centrifugation, resulting in irreversible aggregation. In other words, Cit-Au NPs are not stable for long time and further processing/applications. There are many strategies to improve the colloidal stability of Cit-Au NPs including exchanging citrate molecules with ligands that provide superior stability or specific functionality. Au NPs of 5 and $10 \mathrm{~nm}$ core diameter were used to perform ligand exchange reaction by displacing citrate ions on the gold surface with bis(p-sulfonatophenyl)-phenylphosphine as described previously by Pellegrino et $a^{51,52}$

In order to perform the ligand exchange, bis(p-sulfonatophenyl)phenylphosphine dihydrate dipotassium salt (150 mg (281 $\mu \mathrm{mol}, 1.0$ eq; Strem Chemicals, \#15-0463) was dissolved in $500 \mathrm{~mL}$ suspension of Cit-Au NPs $\left(d_{c}=5 \mathrm{~nm}\left(c_{N P}=8.3 \mathrm{nM}\right)\right.$ or $d_{c}=10 \mathrm{~nm}$ $\left(c_{N P}=9.0 \mathrm{nM}\right), \mathrm{BB}$ I Solutions, \#15702 and \#15703, or synthesized according to §II.2). The resulting solution was stirred at RT for 2 days, cf. Figure III.2.1. After this, the mixture was concentrated to approximately $2 \mathrm{~mL}, c f$. $\S \mathrm{VII} .2$. For that, the solution was centrifuged at $3000 \mathrm{rcf}$ for $5 \mathrm{~min}$ using centrifuge filters (Merck Millipore, $100 \mathrm{kDa}$, $15 \mathrm{~mL}$ ). Next, the concentrated Cit-Au NPs suspension was diluted in phosphine solution ( $3 \mathrm{mg}$ bis( $p$-sulfonatophenyl)phenylphosphine dihydrate dipotassium salt per $10 \mathrm{~mL}$ Milli-Q water) to $15 \mathrm{~mL}$. Then, the solution was concentrated to $2 \mathrm{~mL}$ using centrifuge filters as described previously. Importantly, in order to ensure an excess of phosphine (by decreasing then amount of citrate molecules), the dilution and concentration step was repeated three times. Finally, this leads to phosphine-capped Au NPs (P-Au NPs) that are suspended in $3 \mathrm{mg} / 10 \mathrm{~mL}$ phosphine solution. 


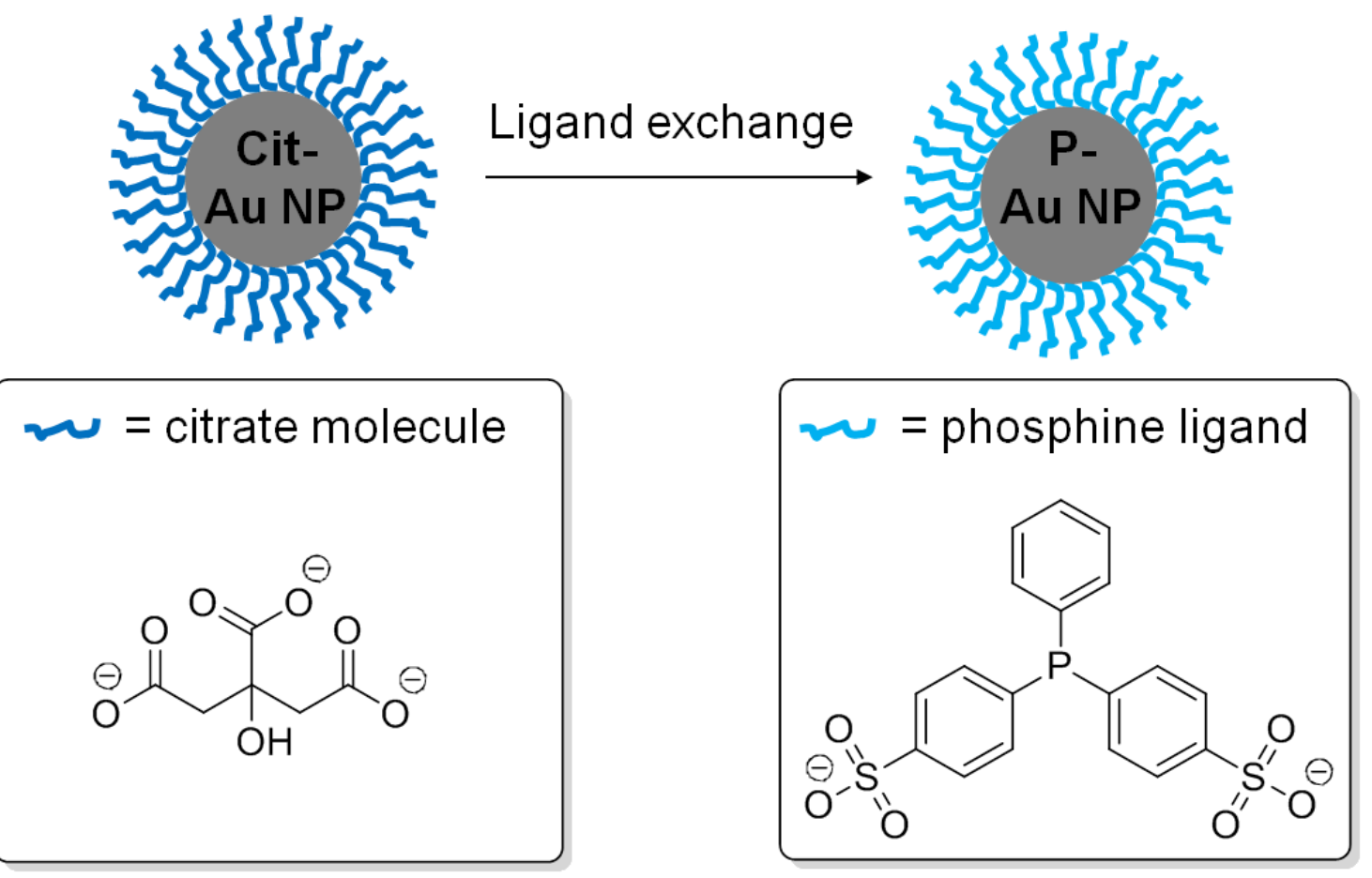

Figure III.2.1. Scheme of the ligand exchange on Cit-Au NPs of different size with bis( $p$ sulfonatophenyl)-phenylphosphine. Spherical Cit-Au NPs of $d_{c}=5$ and $10 \mathrm{~nm}$ core size stabilized by citrate molecules (drawn as dark blue ligands) were modified by bis( $p$ sulfonatophenyl)-phenylphosphine molecules (drawn as light blue ligands). The resulting P-Au NPs have improved colloidal stability.

\section{III.3 Ligand exchange of citrate-capped Au NPs with thiolated polyethyleneglycol}

PEGylation, i.e. coating the surface of NPs with an organic shell composed of polyethylene glycol (PEG), is among the most used strategies to improve the colloidal stability of Au NPs in both, polar and non-polar solvents, due to the amphiphilic character of PEG. Moreover, PEGylation is essential for various biomedical applications as it enhances the biocompatibility of NPs and alters their in vitro cellular interaction and in vivo pharmacokinetics ${ }^{53}$. Usually, linear thiolated PEG polymers with molecular weight in the range of 0.75 to $10 \mathrm{kDa}$ are employed to PEGylate Cit-Au NPs ${ }^{54-56}$ Thiol moieties are necessary to ensure strong attachment of PEG chains to the surface of Au NPs via the strong Au-S bond forming a self-assembled monolayer of PEG on the Au NP surface. In a typical experiment, PEG-SH is dissolved in distilled water (e.g. $10 \mathrm{mg} \cdot \mathrm{mL}^{-1}$ ) and added in excess (ca. 5-15 PEG per $\mathrm{nm}^{2}$ of NP surface $A_{c}$ ) to ensure full coverage of PEG on the surface of Au NPs ${ }^{55}$. After ca. 2 min stirring, $\mathrm{NaOH}(2 \mathrm{M})$ is added dropwise to increase the $\mathrm{pH}$ value to 8-9 (to deprotonate thiol groups in PEG-SH and thus increase their reactivity). Samples are stirred overnight at RT, followed by centrifugation (at least three steps) to collect the PEG-capped Au NPs (PEG-Au NPs). 
Notice that the centrifugal force has to be adapted to $d_{c}$ (bigger colloids require less centrifugal force). For instance for PEG-Au NPs with $d_{c} \approx 14 \mathrm{~nm}, 30 \mathrm{~min}$ at $c a .15,000$ rcf was sufficient to obtain clear supernatants and to pellet all PEG-Au NPs containing excess. Purified PEG-Au NPs can be stored (ideally at $4{ }^{\circ} \mathrm{C}$ ) for extended periods of time (years) without losing their colloidal stability and preserving the initial optical properties (i.e. UV/vis absorption spectrum). Figure III.3.1 illustrates the PEGylation method for Cit-Au NPs with thiolated PEG molecules, resulting in PEG-Au NPs.

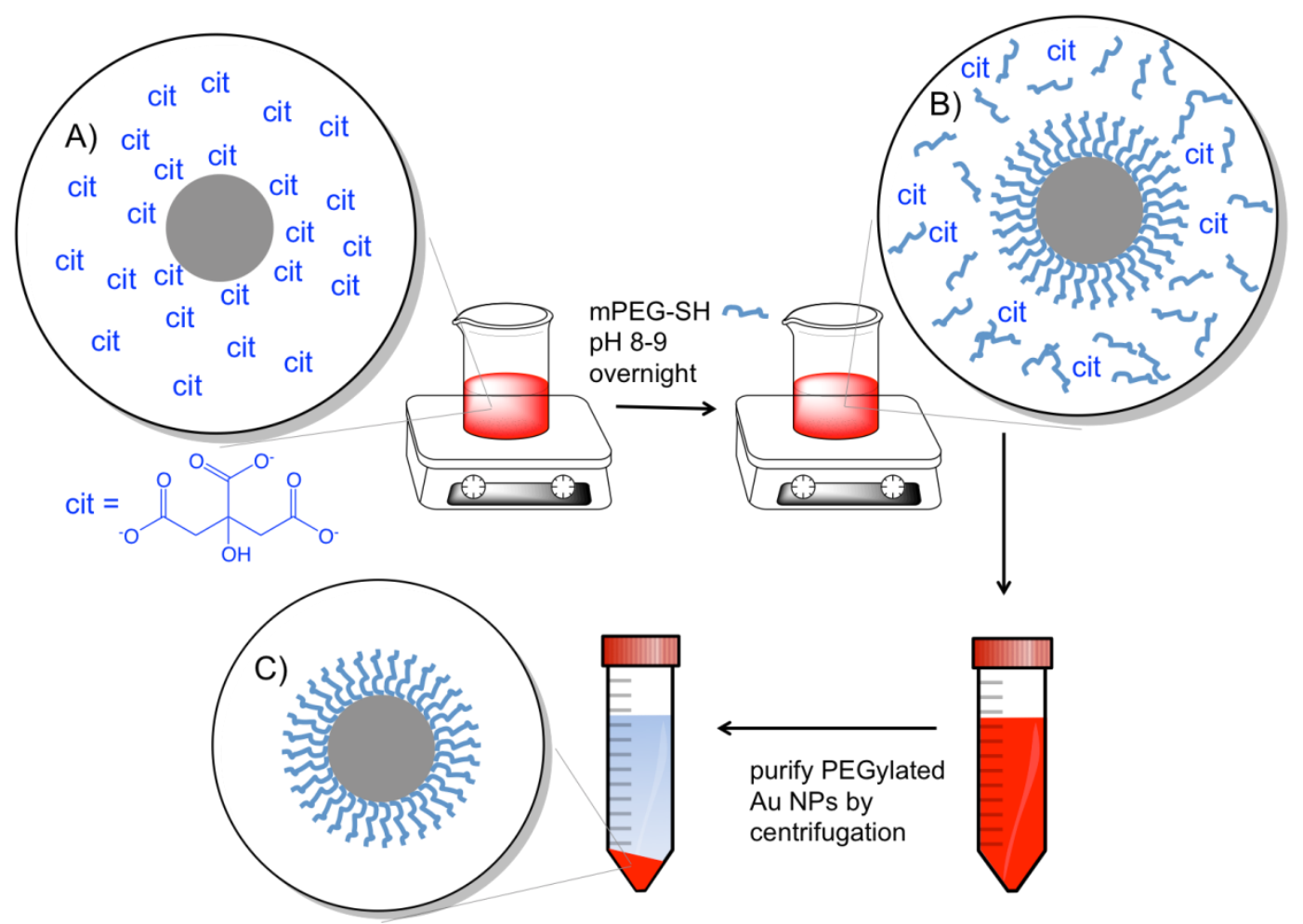

Figure III.3.1. A) Cit-Au NPs in solution with physically adsorbed and excess free citrate molecules. B) Addition of $\mathrm{mPEG}-\mathrm{SH}$ at basic $\mathrm{pH}$ to promote displacement of citrate molecules by MPEG-SH. C) Purification by centrifugation in which PEGylated NPs are purified from free citrate molecules and MPEG-SH in the supernatant. 
IV. Ligand exchange procedures to transfer hydrophilic NPs from aqueous solution to organic solvent and thus to render them hydrophobic

\section{IV.1 General considerations}

IV.2 Ligand exchange on citrate-capped Au NPs with PEG-SH followed by phase transfer of PEG-capped Au NPs to chloroform containing dodecylamine

IV.3 Ligand exchange on CTAB-capped Au NRs with PEG-SH followed by phase transfer of PEG-capped Au NPs to chloroform containing dodecylamine

IV.4 Phase transfer of star-shaped PEG-capped Au NPs to chloroform containing dodecylamine

\section{IV.1 General considerations}

The synthesis of Au NPs with complex shapes and/or large size is usually carried out in aqueous media. However, some applications require hydrophobic Au NPs, for example in the cases the NPs are to be embedded into an organic matrix as in the case of molecular electronics. With this in mind, a significant effort was devoted to developping facile post-synthesis surface functionalization protocols that modify the surface of hydrophilic Au NPs with hydrophobic ligands (surface hydrophobization) ${ }^{57}$. In this section we will describe three examples in which Au NPs were typically prepared in water (Cit-Au NPs, CTAB-Au NRs, and star-shaped PEG-Au NPs as per §II.3), followed by surface PEGylation, which is an intermediate step prior to phase transfer the NPs to organic solvent containing alkylamines ${ }^{56,58}$. We found that PEGylation is crucial to initiate phase transfer without NP aggregation due to the unique solubility of PEG in both, aqueous and organic media, which provides excellent colloidal stability for NPs in both media. For more insights into the mechanism of phase transfer, the original references should be consulted as cited. 


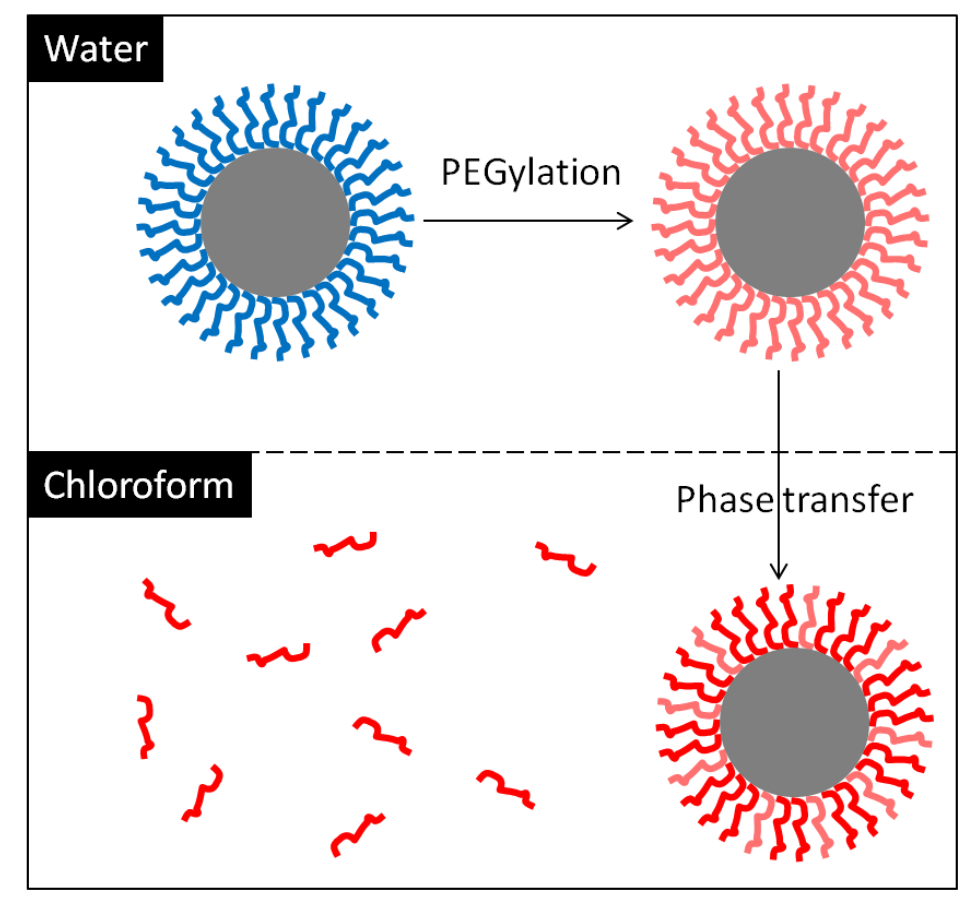

Figure IV.1.1. Hydrophobization of Au NPs via two-stage functionalization: 1) Pegylation in water (upper panel) by displacement of hydrophilic ligands (drawn in blue) by PEG-SH molecules (drawn in light red), followed by phase transfer into chloroform containing alkylamines (drawn in dark red).

\section{IV.2 Ligand exchange on citrate-capped Au NPs with PEG-SH followed by phase transfer of PEG-capped Au NPs to chloroform containing dodecylamine}

Herein, a protocol to transfer Cit-Au NPs, as prepared in §ll.2, from water to chloroform is detailed ${ }^{56}$. As prepared Cit-Au NPs were first coated with mPEG-SH (Mw = 750 Da, Rapp Polymer, \#12750-40), as described in details in §Ill.3, followed by purification and concentration (range of $c a . c_{N P}=50-200 \mathrm{nM}$ in distilled water). Then, typically $1 \mathrm{~mL}$ of the concentrated solution of PEG-Au NPs was mixed with $5 \mathrm{~mL}$ of chloroform (Carl Roth, \#Y015.2) containing DDA as per Table IV.2.1. DDA was added in excess of $\sim 2.5 \cdot 10^{6}$ DDA molecules per NP, in order to promote partial /full exchange of PEG by DDA. This resulted in a two-phase system with the aqueous phase containing the PEGylated NPs on top, and the organic phase with the DDA at the bottom (cf. Figure IV.2.1A). Both phases were mixed by magnetic stirring (ca. $800 \mathrm{rpm}$ ), until the NPs were transferred to the chloroform phase (Figure IV.2.1B). The transfered Au NPs were cleaned twice by centrifugation in order to remove free DDA and PEG molecules (i.e., the supernatant was discarded, and the NP pellet was redispersed in chloroform). The same protocol can be employed to phase transfer Cit-Au NPs with various core diameter as per Table IV.2.1. 


\begin{tabular}{|c|c|c|c|c|}
\hline $\mathbf{d}_{\mathbf{c}}[\mathrm{nm}]$ & $\mathbf{c}_{\mathrm{NP}}[\mathrm{nM}]$ & $\mathbf{c}_{\mathrm{DDA}}[\mathrm{M}]$ & $\mathbf{C}_{\mathrm{PEG}} / \mathbf{c}_{\mathrm{NP}}$ & $\mathbf{C}_{\mathrm{DDA}} / \mathbf{c}_{\mathrm{NP}}$ \\
\hline 5 & 200 & 0.1 & $1 \times 10^{4}$ & $2.5 \times 10^{6}$ \\
\hline 13 & 200 & 0.2 & $2 \times 10^{4}$ & $5 \times 10^{6}$ \\
\hline 20 & 200 & 0.4 & $3 \times 10^{4}$ & $10 \times 10^{6}$ \\
\hline 25 & 150 & 0.4 & $3 \times 10^{4}$ & $13 \times 10^{6}$ \\
\hline 50 & 100 & 0.4 & $20 \times 10^{4}$ & $20 \times 10^{6}$ \\
\hline 100 & 50 & 0.4 & $50 \times 10^{4}$ & $40 \times 10^{6}$ \\
\hline
\end{tabular}

Table IV.2.1. Phase transfer of spherical Cit-Au NPs with various core diameters $\left(d_{c}\right) . c_{N P}$ and $C_{D D A}$ refer to the concentration of NPS and DDA, respectively. The ratios $C_{P E G} / c_{N P}$ and $C_{D D A} / c_{N P}$ describe the number of PEG and DDA molecules added per Au NP, respectively. Detailed procedure to determine Au NPs concentration $c_{N P}$ can be found in sections VIII.2 and IX.2.

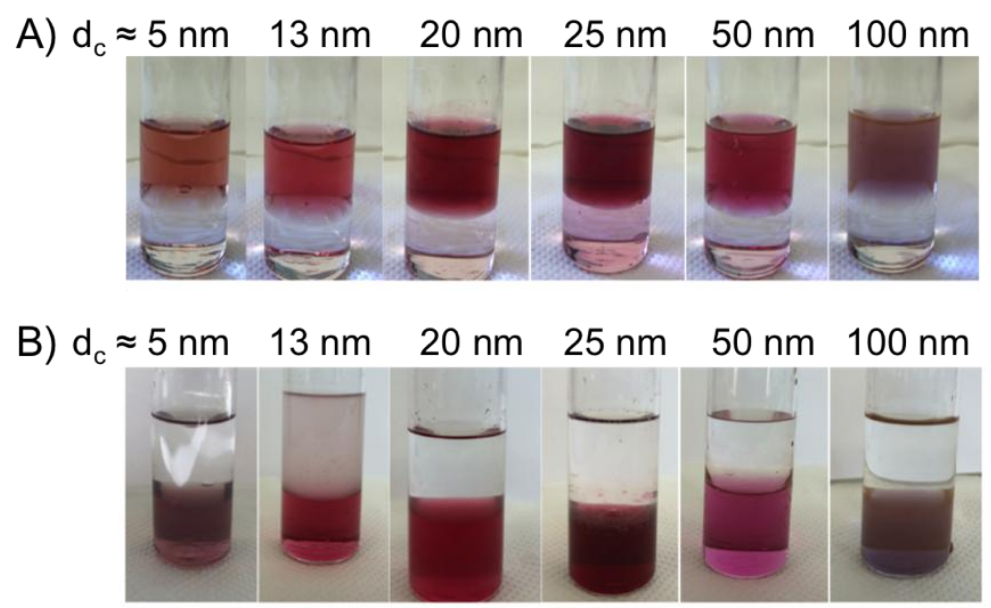

Figure IV.2.1. Phase transfer of PEG-Au NPs from water (upper layers in all vials) to chloroform containing DDT (lower layers in all vials). A) Photographs of biphasic system after ligand exchange of Cit-Au NPs with PEG in water. B) Photographs of biphasic system after phase transfer of PEG-Au NPs to the chloroform layer with DDT. Different vials correspond to Au NPs with different core diameter as labeled.

\section{IV.3 Ligand exchange on CTAB-capped Au NRs with PEG-SH followed by phase transfer of PEG-capped Au NPs to chloroform containing dodecylamine}

CTAB-Au NRs can be transfer to the chloroform phase using a similar protocol as for Cit-Au NPs as described in §IV. $2^{56}$. Initially, free CTAB in excess in "as prepared" CTAB$\mathrm{Au}$ NRs suspensions was removed by centrifugation (5000 rcf, $30 \mathrm{~min}$ ) and the supernatant was discarded. The resultant NP pellets were then resuspended in water 
and another round of purification was performed. To the purified CTAB-Au NRs, PEGSH (mPEG-SH, $M_{W}=750$ Da, Rapp Polymer, \#12750-40) was added as per Table IV.3.1 and mixed for $2 \mathrm{~min}$. Then, the $\mathrm{pH}$ was adjusted to ca. 8-9 by dropwise addition of $\mathrm{NaOH}(2 \mathrm{M})$. The solution was left under stirring at RT overnight, to allow for selfassembly of PEG-SH on the Au NRs to form PEG-Au NRs. The PEG-Au NRs were then purified by centrifugation ( $5000 \mathrm{rcf}, 30 \mathrm{~min}$, twice). A significant decrease in $\zeta$ potential, from $40 \mathrm{mV}$ (CTAB-Au NRs) to $10 \mathrm{mV}$ (PEG-Au NRs) was observed confirming a partial ligand exchange ${ }^{56}$. Finally, a solution of DDA in chloroform (volume ratio 5:1 chloroform to aqueous solution of NPs) was added (cf. Table IV.3.1), leading to a twophase system, which was stirred overnight at RT. After stirring for ca. $12 \mathrm{~h}$, PEG-Au NPs transferred to the chloroform phase (Figure IV.3.1). Au NRs in chloroform were cleaned twice by centrifugation to remove free DDA and PEG molecules, and were finally redispersed in chloroform. The same protocol can be employed to phase transfer CTAB-Au NPs with various aspect ratio as per Table IV.3.1

\begin{tabular}{|c|c|c|}
\hline$\lambda_{\mathrm{SPR}}[\mathrm{nm}]$ & $\mathrm{C}_{\mathrm{PEG}} / \mathrm{c}_{\mathrm{NR}}$ & $\mathrm{C}_{\mathrm{DDA}}[\mathrm{M}]$ \\
\hline 650 & $3 \times 10^{4}$ & 0.4 \\
\hline 790 & $8 \times 10^{4}$ & 0.4 \\
\hline 825 & $8 \times 10^{4}$ & 0.4 \\
\hline 1050 & $8 \times 10^{4}$ & 0.4 \\
\hline
\end{tabular}

Table IV.3.1. Phase transfer of CTAB-Au NRs with various aspect ratio and $\lambda_{S P R} . C_{N R}, C_{P E G}$ and $C_{D D A}$ refer to the concentration of $A U N R S, P E G$, and DDA, respectively. The ratio $C_{P E G} / c_{N R}$ describes the number of PEG molecules added per AU NR. A detailed procedure to determine the Au NR concentration $C_{N P}$ can be found in section VIII.2.
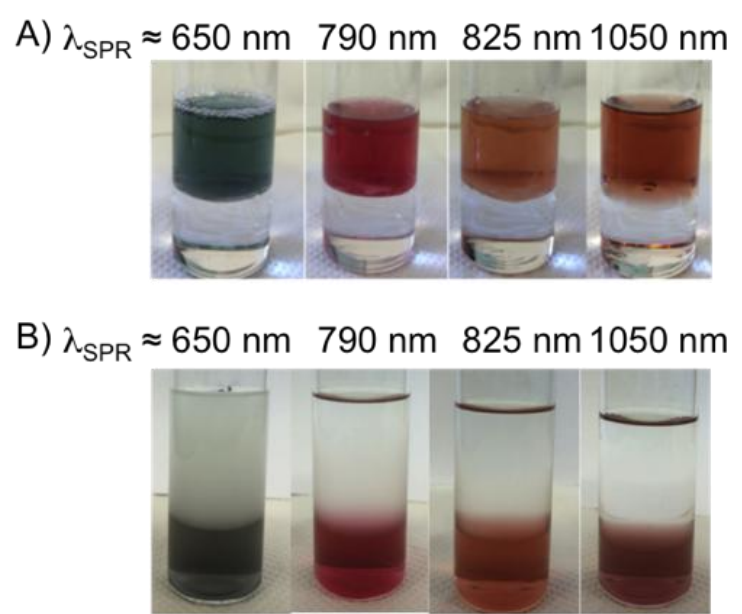
Figure IV.3.1. Phase transfer of PEG-Au NRs from water (upper layers in all vials) to chloroform containing DDT (lower layers in all vials). A) Photographs of the biphasic system after ligand exchange of CTAB-Au NRs with PEG in water. B) Photographs of the biphasic system after phase transfer of PEG-Au NRs to the chloroform layer with DDT. Different vials correspond to Au NRs with different aspect ratio and $\lambda_{S P R}$ as labeled.

\section{IV.4 Phase transfer of star-shaped PEG-capped Au NPs to chloroform containing dodecylamine}

The protocol in $\$ 11.5$ describes the synthesis of star-shaped Au NPs, which are capped with PEG molecules as prepared. Thus, these NPs can be directly transferred from aqueous media to chloroform containing DDA (0.2 M), similar to the phase transfer of PEG-Au NPs and PEG-Au NRs as detailed in §IV.2 and §IV.3. The phase transfer is shown in Figure IV.4.1.

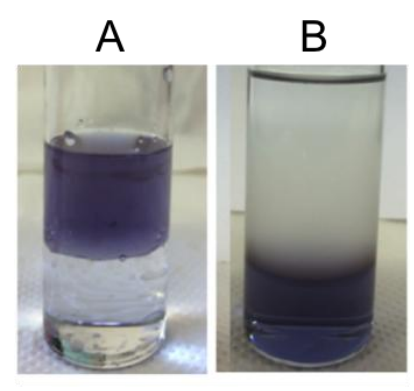

Figure IV.4.1. Phase transfer of star-shaped Au NPs from water (upper layers) to chloroform contains DDT (lower layers). Photographs of biphasic system before (A) and after (B) phase transfer from water to chloroform. 
V. Ligand exchange procedures to transfer hydrophobic NPs into aqueous solution and thus to render them hydrophilic

\section{V.1 General considerations}

V.2 Ligand exchange on DDT-Au NPs with 3-mercaptopropionic acid (MPA) or 11mercaptoundecanoic acid (MUA)

V.3 Phase transfer of DDT-Ag NPs from chloroform to water using 3mercaptopropionic acid (MPA) or 11-mercaptoundecanoic acid (MUA)

\section{V.1 General considerations}

For most biological application dispersion of NPs in aqueous phase is required. Thus, proper surface modification of hydrophobic NPs, which have been originally synthesized in organic solvent, is needed to allow their stable dispersion in aqueous media. Ligand exchange is a powerful approach to displace the original hydrophobic ligands by hydrophilic versions ${ }^{19,59}$. This section describes procedures to modify the surface of hydrophobic Au NPs and Ag NPs with hydrophilic ligands.

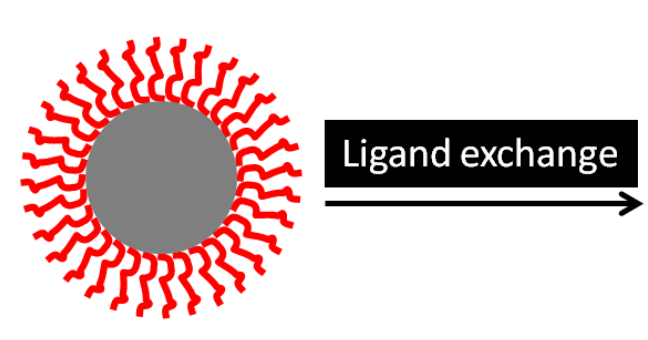

Hydrophobic AuNPs

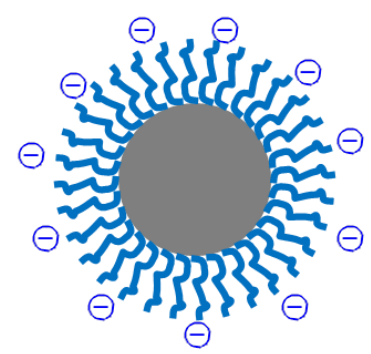

Hydrophilic AuNPs

HS 1-Dodecanethiol<smiles>CC(C)(C)OOC(=O)CCS</smiles>

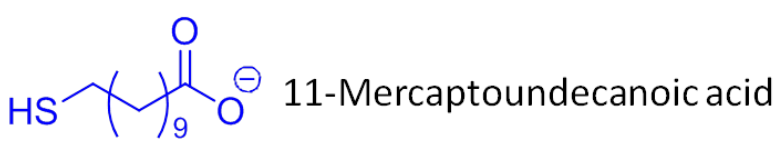

Figure V.1. Ligand exchange on hydrophobic NPs (capped with hydrophobic ligands drawn in red) with hydrophilic ligands (drawn in blue). The chemical structures of 1dodecanethiol, 3-mercaptopropionic acid, and 11-mercaptoundecanoic acid are shown.

V.2 Ligand exchange on DDT-Au NPs with 3-mercaptopropionic acid (MPA) or 11mercaptoundecanoic acid (MUA) 
Here we describe the detailed procedure for synthesizing Au NPs, protected by weakly bound ligands and how to perform the following ligand exchange to mercaptocarbonic acids like mercaptopropionic acid (MPA) or mercaptoundecanoic acid (MUA), which due to their mercapto-groups are strong ligands, $c f$. Figure V.2.1. The protocol for synthesizing precursor Au NPs stabilized by hexanoic or decanoic acid (cf. §l.2), as well as the additional ligand exchange to MPA or MUA was previously described and we refer to the original protocols for more information ${ }^{19,60}$.

Ligand exchange on DDT-Au NPs ( $4 \mathrm{~nm}$ ) with mercaptopropionic acid: To DDT-Au NPs $\left(\mathrm{d}_{\mathrm{c}}=4 \mathrm{~nm}\right.$ ) in chloroform (as synthesized per §I.2), MPA dissolved in $\mathrm{CH}_{2} \mathrm{CL}_{2}(500 \mu \mathrm{L}$ in $2 \mathrm{~mL}$ ) were added, and the mixture was left to react in an ultrasonic bath for $90 \mathrm{~min}$ at RT to allow for ligand exchange. The MPA-Au NPs were then transferred into a $50 \mathrm{~mL}$ centrifuge tube, $20 \mathrm{~mL}$ of methanol were added, and the mixture was centrifuged at $3000 \mathrm{rcf}$ for $5 \mathrm{~min}$. The supernatant was removed and the sedimented Au NPs were resuspended in $20 \mathrm{~mL}$ of methanol and centrifuged. The washing procedure was done 4 times in total. After the last washing step, the supernatant was discarded and the Au NP pellet (at the bottom of the centrifugation tube) was dispersed in $15 \mathrm{~mL}$ of Milli-Q water, which led to a dark red solution. Finally, the MPA-Au NPs were washed twice using centrifuge filters (Merck Millipore, $100 \mathrm{kDa}, 15 \mathrm{~mL}$ ) and a speed of $3000 \mathrm{rcf}$ for 5 min to get rid of free MPA (cf. §VII.2).

Ligand exchange on DDT-Au NPs $(7 \mathrm{~nm})$ with mercaptoundecanonic acid: To DDT-Au $\operatorname{NPs}\left(d_{c}=7 \mathrm{~nm}\right)$ in chloroform (as synthesized per §l.2), $91 \mathrm{mg}(417 \mu \mathrm{mol})$ of MUA were added, and the resulting mixture was first stirred at $60^{\circ} \mathrm{C}$ for $30 \mathrm{~min}$ and then put into an ultrasonic bath at RT for another hour to allow for ligand exchange. MUA-Au NPs were precipitated by the addition of $40 \mathrm{~mL}$ methanol, transferred into a centrifuge tube, and sedimented at $3000 \mathrm{rcf}$ for $10 \mathrm{~min}$ to collect the MUA-Au NPs. The supernatant was discarded and the solid Au NP pellet was resuspended in another $40 \mathrm{~mL}$ of methanol, and again centrifuged. This washing procedure was repeated 3 times and the MUA-Au NPs were finally purified by column chromatography using a Sepharose (CL-4B) column and $25 \mathrm{mM}$ aqueous sodium hydroxide as mobile phase (cf. §VII.3).

\section{V.3 Phase transfer of DDT-Ag NPs from chloroform to water using 3- mercaptopropionic acid (MPA) or 11-mercaptoundecanoic acid (MUA)}

This protocol implies the phase transfer of DDT-Ag NPs with MPA or MUA to render Ag NPs with hydrophilic surface, and thus to resuspend them in aqueous solution with satisfactory colloidal stability ${ }^{61}$. Briefly, in a $250 \mathrm{~mL}$ round bottom flask, DDT-Ag NPs in 
chloroform (10 mL of $0.5 \mathrm{mg} / \mathrm{mL}$, cf. §l.3) were placed. MPA (7.3 mmol, $636 \mu \mathrm{L}$ ) or MUA (7.3 mmol, $1.59 \mathrm{~g}$ ) were dissolved in $130 \mathrm{~mL}$ Tris-Borate-EDTA (TBE) buffer (0.5x) (Sigma-Aldrich, \#T3913). In case of MUA, the buffer mixture had to be dissolved by placing it into an ultrasonic bath for $45 \mathrm{~min}$. Then aqueous buffer solution was then added to the chloroform organic phase, and both phases were mixed thoroughly until a phase transfer of Ag NPs to the aqueous layer at the top of the vial was observed. The two phases were then transferred into a $40 \mathrm{~mL}$ glass vial and centrifuged at $900 \mathrm{rcf}$ for 20 min to precipitate the excess ligands as white solid on the bottom of the vial, and simultaneously to separate the two liquid layers. The aqueous phase, including the MPA-Ag NPs or MUA-Ag NPs on top of the biphasic system was removed by a pipette and further washed three times with Milli-Q water using centrifuge filters (Merck Millipore, $100 \mathrm{kDa}, 15 \mathrm{~mL}$ ) at $3000 \mathrm{rcf}$ for $5 \mathrm{~min}$.

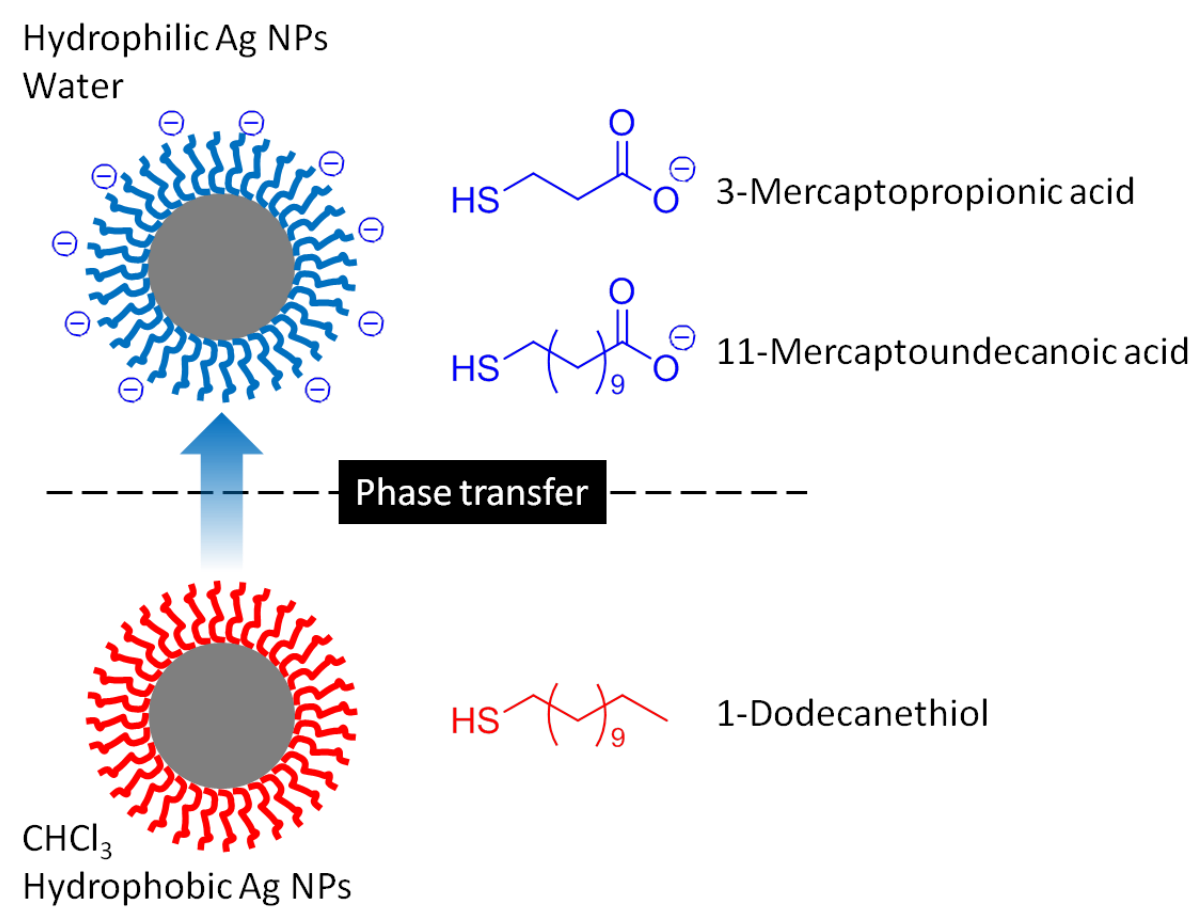

Figure V.3.1. Phase transfer of DDT-Ag NPs from chloroform to water using 3mercaptopropionic acid (MPA) or 11-mercaptoundecanoic acid (MUA). 
VI. Polymer coating to transfer hydrophobic NPs into aqueous solution and thus to render them hydrophilic

\section{VI.1 General considerations}

VI.2 Synthesis of the amphiphilic polymer: poly(isobutylene-alt-maleic anhydride)graft-dodecyl, PMA-g-dodecyl

VI.3 Phase transfer of alkanethiol-capped NPs from chloroform to water using PMAg-dodecyl polymer

\section{VI.1 General considerations}

This section describes the phase transfer of hydrophobic alkanethiol-capped NPs to aqueous phase by overcoating the NPs with an amphiphilic polymer that interacts strongly with the self-assembled alkane monolayer on the NPs' surface and provides a hydrophilic character that enables efficient transfer of NPs from organic solvent to water phase (cf. Figure VI.1.1). In another words, the hydrophobic NPs are encapsulated inside the hydrophobic cavity of the polymer micelles with independence on the type of the inorganic core material ${ }^{62,63}$.
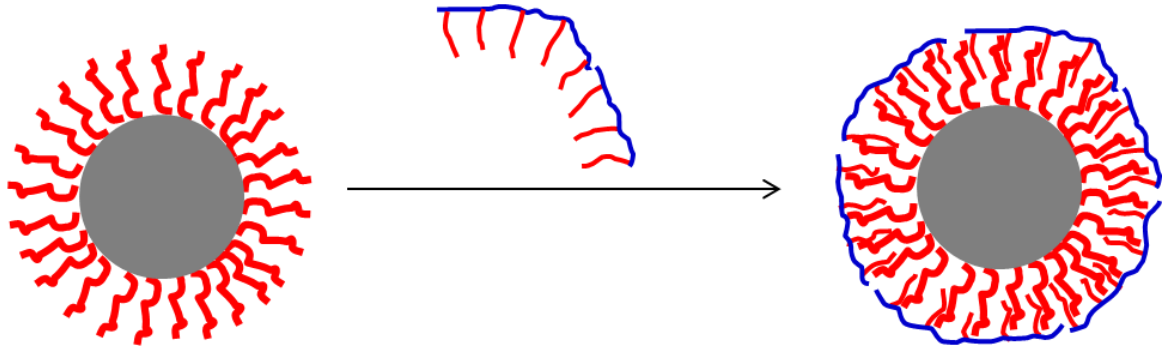

Figure VI.1.1. NP cores (drawn in gray) that are capped with hydrophobic ligands (drawn in red) are modified with an amphiphilic polymer (hydrophilic backbone drawn in blue and hydrophobic side chains drawn in red). The NP core is embedded in the resulting polymer micelle, resulting in a hydrophilic nanoparticle surface.

VI.2 Synthesis of the amphiphilic polymer: poly(isobutylene-alt-maleic anhydride)graft-dodecyl, PMA-g-dodecyl

In principle, there are many potential amphiphilic polymers which can be used for polymer coating of NPs. In this section emphasis is given on an amphiphilic polymer which is based on a backbone of poly(isobutylene-alt-maleic anhydride), functionalized with dodecylamine, yielding dodecylamine hydrophobic side chains through formation 
of amide bonds upon reaction with the maleic anhydride rings (PMA). The anhydride rings that have not been reacted with dodecylamine open in contact with water, forming negatively charged carboxyl groups, and thus providing colloidal stability to the NPs in aqueous solutions. This chemistry allows for preparation of the amphiphilic polymers with additional functionalities (e.g. by attaching organic fluorophores) ${ }^{64}$.

The synthetic PMA has been reported in previous work ${ }^{10,65}$. A $250 \mathrm{~mL}$ round bottom flask received $3.084 \mathrm{~g}(20 \mathrm{mmol}$ expressed as monomer, $c f$. Figure VI.2.1) poly(isobutylene-alt-maleic anhydride) (average $M_{w} \sim 6,000 \mathrm{~g} / \mathrm{mol}$ of whole polymer, molecular weight of one PMA monomer unit $\mathrm{M}_{\mathrm{PMA}} \sim 154 \mathrm{~g} / \mathrm{mol}$, Sigma, \#531278). Then, $2.70 \mathrm{~g}$ (15 mmol) of dodecylamine (DDA, $\geq 98 \%$, Fluka, \#44170) were dissolved in 100 $\mathrm{mL}$ of anhydrous tetrahydrofuran (THF, $\geq 99.9 \%$, Aldrich, \#186562). Note, each polymer monomer unit comprises an anhydride ring. The ratio of dodecylamine / anhydride rings was chosen in a way that $R_{\text {chain } / \mathrm{mon}}=75 \%$ (15 mmol out of $20 \mathrm{mmol}$ ), to allow for $75 \%$ anhydride rings coupled with dodecylamine, and $25 \%$ intacted anhydride rings. It is very important to use anhydrous organic solvents in order to maintain the maleic anhydride rings active during the synthesis, i.e. to prevent hydrolysis. This solution was then added to the poly(isobutylene-alt-maleic anhydride), and the mixture was sonicated for ca. $20 \mathrm{~s}$, followed by heating to $55-60{ }^{\circ} \mathrm{C}$ for $3 \mathrm{~h}$ under stirring. This mixture was initially cloudy and only after a while of heating it turned transparent. Next, the solution was concentrated to $30-40 \mathrm{~mL}$ by evaporation of THF under reduced pressure in a rotary evaporator, and the mixture was heated under reflux overnight. Finally, the solvent was completely evaporated under reduced pressure in a rotary evaporator and the dried polymer powder was dissolved in $\mathrm{V}_{\mathrm{P}_{-} \text {sol }}=$ $40 \mathrm{~mL}$ anhydrous chloroform ( $\geq 99 \%$, Sigma, \#372978), yielding a solution $0.5 \mathrm{M}$ in monomer concentration, $c_{p}$. It is important to keep this polymer solution under anhydrous conditions that the remaining maleimide rings do not yet hydrolyze.

The final molar mass of one polymer unit $M_{P}$ comprises the molar mass $M_{P M A}$ of one PMA monomer and the molar mass $M_{D D A}=185 \mathrm{~g} / \mathrm{mol}$ of one DDA side chain times the number of side chains per monomer, see Equation VI.2.1.

$$
M_{P}=M_{P M A}+R_{\text {chain } / \text { mon }} \times M_{D D A}
$$

For $R_{\text {chain } / \text { mon }}=0.75$ the value of $M_{p}=154 \mathrm{~g} / \mathrm{mol}+0.75 \times 185 \mathrm{~g} / \mathrm{mol} \approx 293 \mathrm{~g} / \mathrm{mol}$ is obtained. 
A

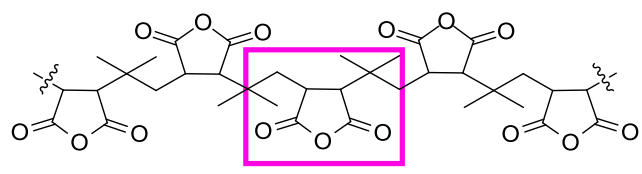

B

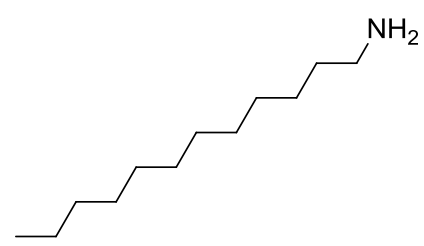

C

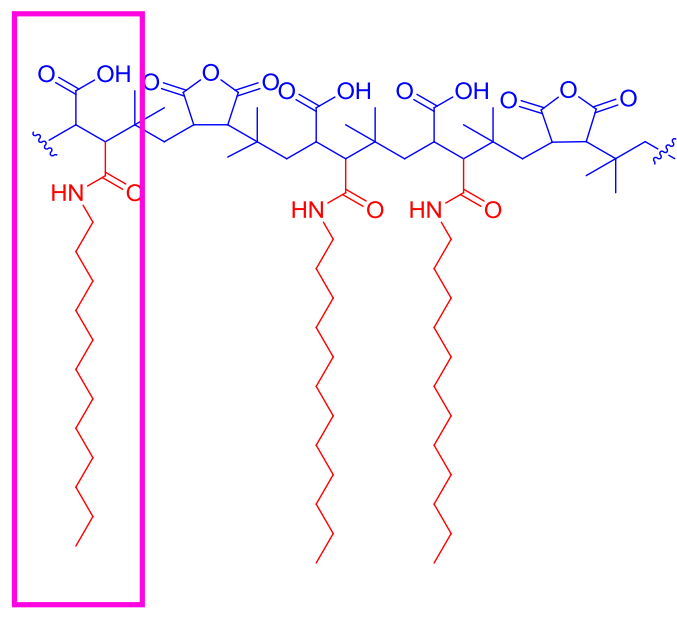

Figure VI.2.1. Synthesis of poly(isobutylene-alt-maleic anhydride)-graft-dodecyl, PMA-g-dodecyl A) Poly(isobutylene-alt-maleic anhydride) is used as hydrophilic backbone (the purple box shows one monomer unit). B) Dodecylamine is used as hydrophobic side chain. The amphiphilic polymer was obtained by reaction of the hydrophilic backbone with hydrophobic side chains. C) Structure of the amphiphilic polymer (PMA-g-dodecyl). The purple box in C shows a monomer unit with attached side chain (of molar mass $M_{P}$ ). The hydrophobic and hydrophilic parts are drawn in red and blue, respectively

During the polymer synthesis molecules that are soluble in THF and which bear a $-\mathrm{NH}_{2}$ group can be integrated into the polymer. This can be done by adding those aminecontaining molecules (like dyes or spacer molecules like polyethylene glycol) together with the dodecylamine to the PMA. By this $\mathrm{R}_{\text {chain } / \mathrm{mon}}=75 \%$ of the anhydride rings will react with the $\mathrm{NH}_{2}$ group of the dodecylamine, as described above and some of the remaining PMA monomers with the additional functional molecules. Typically not more than $R_{\text {func/mon }}=5 \%$ of the anhydride rings should be modified with functional molecules, as otherwise the polymer coating procedure as described in $\S \mathrm{VI} .3$ may no longer work. A list with modified polymers that has been reported in literature is provided in §VI.3.

\section{VI.3 Phase transfer of alkanethiol-capped NPs from chloroform to water using PMA- g-dodecyl polymer.}

DDT-Au NPs in chloroform was mixed with PMA-g-dodecyl dissolved in chloroform. The mixture was stirred manually for $5 \mathrm{~min}$ at RT and then the solvent (i.e. chloroform) was completely evaporated in a rotary evaporator under heating at $40{ }^{\circ} \mathrm{C}$ to force the 
polymer to wrap around the NPs, as illustrated in Figure VI.3.1. A few $\mathrm{mL}$ of anhydrous chloroform were added to the flask to reconstitute the solid film and again the solvent was removed under reduced pressure. This step was carried out 3 times in order to obtain a homogeneous coating. After the last step, the remaining solid film in the flask film was reconstituted in alkaline sodium borate buffer $(50 \mathrm{mM}, \mathrm{pH} 12$ adjusted with $\mathrm{NaOH}$ ) under vigorous stirring until the solution turned clear, $c f$. Figure VI.3.2. Sonication can be optionally used to facilitate the dispersion. In the last step, the remaining anhydride rings get hydrolyzed in alkaline condition resulting in carboxylate groups that allow reconstitution in water with excellent colloidal stability. This approach can be employed to transfer various types of NPs with different sizes from water to chloroform as per Table VI.3.1.

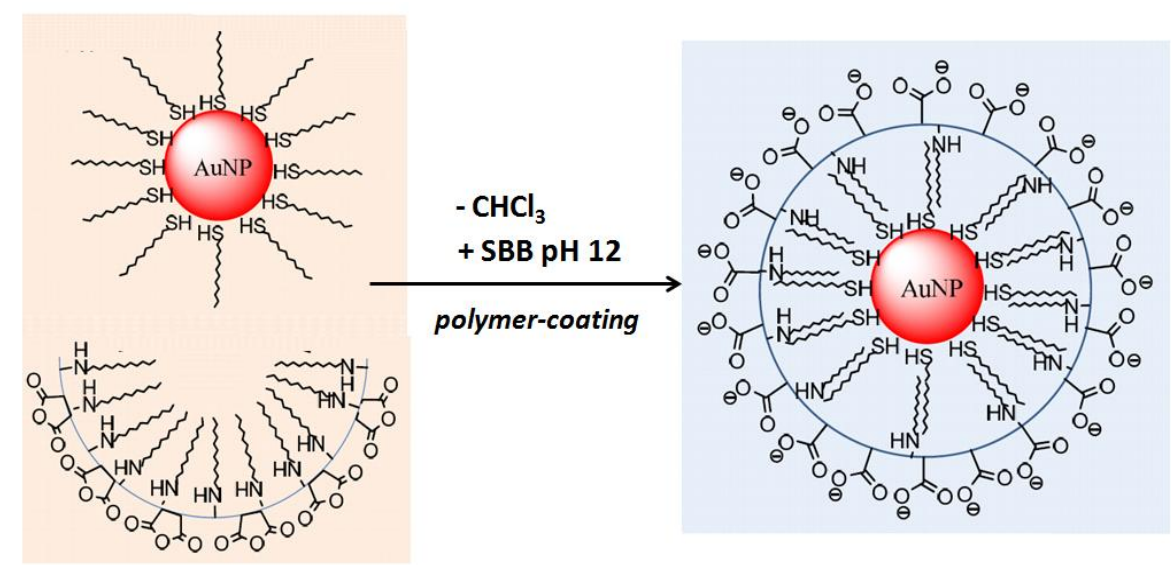

Figure VI.3.1. Phase transfer of DDT-Au NPs from chloroform (left) to water (right) using PMA-g-dodecyl polymer.

A key point in this procedure is the calculation of the amount of polymer $N_{p}$ (in terms of monomer units), which needs to be added to the NPs. The amount of polymer per NP scales with the effective surface area $A_{\text {eff }}$ of one NP, and with the amount of NPs. In case of spherical NPs $A_{\text {eff }}$ is given as the surface of a sphere (cf. Figure I.1.1):

$$
A_{\text {eff }}=4 \pi \times\left(\frac{d_{\text {eff }}}{2}\right)^{2}=\pi \times d_{\text {eff }}^{2}
$$

Nanorods were considered as cylinders with diameter $d_{\text {eff }}$ and length $L_{\text {eff }}$ to simplify the calculations (cf. Figure 1.2.1), and thus the effective surface of one rod-shaped NP becomes

$$
A_{\text {eff }}=2 \pi \times\left(\frac{d_{e f f}}{2}\right) \times L_{e f f}=\pi \times d_{e f f} \times L_{e f f}
$$


In a solution with volume $\mathrm{V}_{\mathrm{NP}_{-} \text {sol }}$ and NP concentration $\mathrm{c}_{\mathrm{NP}}$ the number of moles of NPS is $n_{N P}=c_{N P} \cdot V_{N P \_s o l}$, i.e. the total number of NPs $N_{N P}=n_{N P} \times N_{A}\left(N_{A}=\right.$ Avogadro's number). The total effective surface area of all NPs in solution thus is

$$
A_{\text {total_eff }}=N_{N P} \times A_{\text {eff }}=c_{N P} \times V_{N_{-} \_s o l} \times N_{A} \times A_{\text {eff }}
$$

The number of monomer units that needs to be added per $\mathrm{nm}^{2}$ of effective surface area $\left(\mathrm{R}_{\mathrm{P} / \mathrm{Area}}\right)$ is an important value that should be determined experimentally for any polymer coating protocol, as it may depend on the core material, core diameter, surface capping, etc. of the NPs. The number of polymer monomers $N_{p}$ that needs to be added to the NP suspension thus is

$$
\mathrm{N}_{\mathrm{P}}=\mathrm{R}_{\mathrm{P} / \text { Area }} \times \mathrm{A}_{\text {total_eff }}
$$

For a polymer stock solution of monomer concentration $C_{P}$ thus the volume $V_{P_{-} \text {sol }}$ needs to be added to the NP suspension.

$$
\mathrm{V}_{\mathrm{P}_{\text {_sol }}}=\frac{\mathrm{n}_{\mathrm{P}}}{\mathrm{c}_{\mathrm{P}}}=\frac{\left(\frac{\mathrm{N}_{\mathrm{P}}}{\mathrm{N}_{\mathrm{A}}}\right)}{\mathrm{c}_{\mathrm{P}}}=\frac{\left(\mathrm{R}_{\mathrm{P} / \text { Area }} \times \mathrm{A}_{\text {total eff }}\right)}{\mathrm{N}_{\mathrm{A}} \times \mathrm{c}_{\mathrm{P}}}=\frac{\left(\mathrm{R}_{\mathrm{P} / \text { Area }} \times \mathrm{A}_{\mathrm{eff}} \times \mathrm{c}_{\mathrm{NP}} \times \mathrm{V}_{\mathrm{NP} \_ \text {sol }}\right)}{\mathrm{c}_{\mathrm{P}}} \quad \text { (Equation VI.3.4) }
$$

The polymer coating procedure should be carried out in sufficiently diluted NP suspension. In the case of NPs with $d_{c}<10 \mathrm{~nm}$ we typically uses a NP concentration of around $\mathrm{c}_{\mathrm{NP}}=1 \mu \mathrm{M}$. In case of bigger NPs, lower NP concentrations should be used.

To give an example of the above calculation: A batch of hydrophobic as Au NPs synthesized according to $§ I .2$ have a core diameter of $d_{c}=4.2 \mathrm{~nm}$, and a ligand length of dodecanethiol of $I_{\text {ligand }}=1 \mathrm{~nm}$ was assumed. The Au NPs were suspended at a concentration of $\mathrm{C}_{\mathrm{NP}}=1 \mu \mathrm{M}$ in a volume of $\mathrm{V}_{\mathrm{NP}_{-} \text {sol }}=7.1 \mathrm{~mL}$. For the polymer coating procedure the amount of polymer added per effective NP surface was chosen as $\mathrm{R}_{\mathrm{P} / \mathrm{Area}}$ $=100 \mathrm{~nm}^{-2}$. The polymer was dissolved in a stock solution with monomer concentration $c_{p}=0.05 \mathrm{M}$. In this way with help of Equation I.1.1, Equation VI.3.1, and Equation VI.3.4 the amount $\mathrm{V}_{\mathrm{P}_{\text {_sol }} \text { ol }}$ of polymer solution, which needs to be added to the NP suspension of volume $V_{N P}$ can be calculated:

$$
\begin{aligned}
d_{\text {eff }} & =d_{c}+2 \times l_{\text {ligand }}=4.2 \mathrm{~nm}+(2 \times 1 \mathrm{~nm})=6.2 \mathrm{~nm} \\
A_{\text {eff }}= & \pi \times d_{\text {eff }}^{2}=\pi \times(6.2 \mathrm{~nm})^{2}=121 \mathrm{~nm}^{2} \\
V_{P_{-} \text {sol }} & =\left(R_{P / A r e a} \times A_{\text {eff }} \times C_{N P} \times V_{N P \_s o l}\right) / c_{P}=\left(100 n^{-2} \times 121 n^{2} \times 1 \mu M \times 7.1 \mathrm{~mL}\right) / 0.05 \mathrm{M} \\
& =1.7 \mathrm{~mL}
\end{aligned}
$$


A list of polymer coating conditions for a variety of NP materials is given in Table VI.3.1. Parameters for NPs coated with functionalized polymers are enlisted in Table VI.3.2 and Table VI.3.3.

A

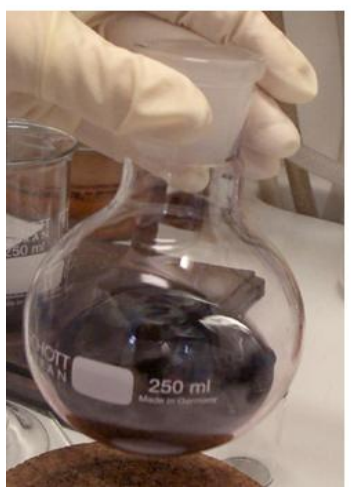

B

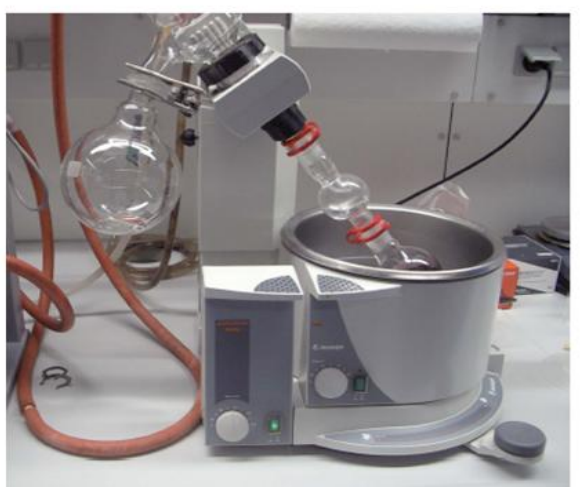

C

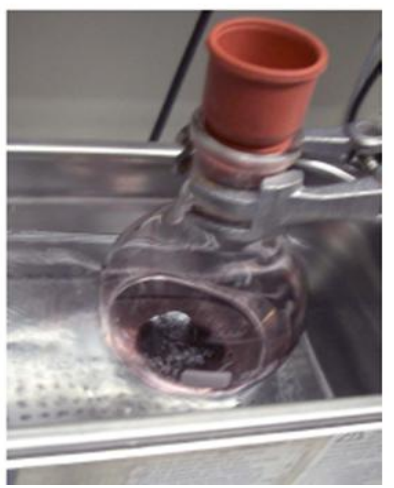

Figure VI.3.2. Phase transfer of DDT-Au NPs $\left(d_{c}=4.2 \mathrm{~nm}\right)$ from chloroform to water using PMA-g-dodecyl polymer. Photographs of: A) DDT-Au NPs in chloroform after addition of the polymer solution dissolved in chloroform. B) Chloroform is evaporated using a rotary evaporator. C) Sonication after the addition of sodium borate buffer ( $\mathrm{pH}$ 12).

\begin{tabular}{|c|c|c|c|c|c|c|c|c|}
\hline $\begin{array}{c}\text { NP } \\
\text { material }\end{array}$ & Shape & $\begin{array}{c}\mathrm{d}_{\mathrm{c}} \\
{[\mathrm{nm}]}\end{array}$ & $\begin{array}{c}\mathrm{L}_{\mathrm{c}} \\
{[\mathrm{nm}]}\end{array}$ & $\begin{array}{l}\text { I ligand } \\
{[\mathrm{nm}]}\end{array}$ & $\begin{array}{l}\text { Synthesis } \\
\text { described } \\
\text { in }\end{array}$ & $\mathrm{R}_{\text {chain/mon }}$ & $\begin{array}{l}\mathrm{R}_{\mathrm{P} / \text { Area }} \\
{\left[\mathrm{nm}^{-2}\right]}\end{array}$ & Reference \\
\hline $\mathrm{Au}$ & Sphere & 4.2 & - & 1.0 & $\S 1.2$ & $75 \%$ & 100 & 10 \\
\hline $\mathrm{Au}$ & Sphere & 25 & - & 1.0 & $\S ા I .2, \S$ §IV.2 & $75 \%$ & 3000 & 56 \\
\hline $\mathrm{Au}$ & Sphere & 50 & - & 1.0 & §II.2, §IV.2 & $75 \%$ & 3000 & 56 \\
\hline $\mathrm{Au}$ & Sphere & 50 & - & 1.0 & §II.2, §IV.2 & $75 \%$ & 3000 & 56 \\
\hline $\mathrm{Au}$ & Sphere & 100 & - & 1.0 & §ાI.2, §IV.2 & $75 \%$ & 4000 & - \\
\hline $\mathrm{Au}$ & Rod & 15 & 40 & 1.0 & §II.4, §IV.3 & $75 \%$ & 3000 & - \\
\hline $\mathrm{Au}$ & Rod & 15 & 63 & 1.0 & §lI.4, §IV.3 & $75 \%$ & 3000 & 56 \\
\hline $\mathrm{Au}$ & Rod & 30 & 100 & 1.0 & §II.4, §IV.3 & $75 \%$ & 3000 & - \\
\hline $\mathrm{Au}$ & Rod & 12 & 83 & 1.0 & §II.4, §IV.3 & $75 \%$ & 3000 & 56 \\
\hline $\mathrm{Ag}$ & Sphere & 4.2 & & 1.0 & $\S 1.3$ & $75 \%$ & 100 & 61 \\
\hline FePt & Sphere & 4 & - & 1.0 & $\S 1.4$ & $75 \%$ & 100 & 66 \\
\hline $\mathrm{Fe}_{3} \mathrm{O}_{4}$ & Sphere & 4 & - & 1.0 & $\S 1.5$ & $75 \%$ & 100 & - \\
\hline $\mathrm{CdSe} / \mathrm{ZnS}$ & Sphere & $2-10$ & & 1.2 & $\S । .1 .7$ & $75 \%$ & 100 & 63,67 \\
\hline
\end{tabular}

Table VI.3.1. List of parameters used to overcoat various NPS with PMA-g-dodecyl polymer. In some of the examples ${ }^{56}$ the original NPs were not capped with hydrophobic ligands, and thus were capped with DDT previous to the polymer coating procedure. 


\begin{tabular}{|c|c|c|c|c|c|c|c|c|c|c|c|c|}
\hline $\begin{array}{l}\text { NP core } \\
\text { material }\end{array}$ & Shape & $\mathrm{d}_{\mathrm{c}}[\mathrm{nm}]$ & $\mathrm{L}_{\mathrm{c}}[\mathrm{nm}]$ & $\mathrm{I}_{\text {ligand }}[\mathrm{nm}]$ & $\mathrm{R}_{\text {chain/mon }}$ & $\mathrm{R}_{\text {cross/mon }}$ & $\mathrm{R}_{\text {func/mon }}$ & Added functionality & $\begin{array}{l}\text { R } / \text { Area } \\
{\left[\mathrm{nm}^{-2}\right]}\end{array}$ & $d_{h}[n m]$ & $\zeta[\mathrm{mV}]$ & Ref. \\
\hline $\mathrm{Au}$ & Sphere & $3.2 \pm 0.5$ & - & - & $75 \%$ & 0 & 0 & - & $100-200$ & 13.9 & - & 65 \\
\hline $\mathrm{Au}$ & Sphere & $3.2 \pm 0.5$ & - & - & $100 \%$ & 0 & 0 & - & $100-200$ & - & - & 65 \\
\hline $\mathrm{Au}$ & Sphere & $3.2 \pm 0.5$ & - & - & $100 \%$ & 0 & 0 & - & $100-200$ & - & - & 65 \\
\hline $\mathrm{Au}$ & Sphere & $3.2 \pm 0.5$ & - & - & $100 \%$ & 0 & 0 & - & $100-200$ & - & - & 65 \\
\hline $\mathrm{Au}$ & Sphere & $3.2 \pm 0.5$ & - & - & $100 \%$ & 0 & 0 & - & $100-200$ & 11.4 & - & 65 \\
\hline $\mathrm{Au}$ & Sphere & 4.0 & - & 1.0 & $100 \%$ & $7 \%$ & 0 & - & 147,295 & - & - & 63 \\
\hline $\mathrm{Au}$ & Sphere & 4.0 & - & 1.0 & $100 \%$ & $8 \%$ & 0 & - & 310 & - & - & 63 \\
\hline $\mathrm{Au}$ & Sphere & 4.0 & - & 1.0 & $100 \%$ & $10 \%$ & 0 & - & 92,100 & - & - & 63 \\
\hline $\mathrm{Au}$ & Sphere & 4.0 & - & - & $100 \%$ & $10 \%$ & 0 & - & 100 & - & - & 17 \\
\hline $\mathrm{Au}$ & Sphere & 4.0 & - & - & $100 \%$ & $10 \%$ & 0 & - & 100 & - & - & 17 \\
\hline $\mathrm{Au}$ & Sphere & 4.0 & - & 1.0 & $75 \%$ & 0 & 0 & - & 100 & - & - & 10 \\
\hline $\mathrm{Au}$ & Sphere & 4.0 & - & 1.0 & $75 \%$ & 0 & $1 \%$ & Fluoresceinamine & 100 & - & - & 10 \\
\hline $\mathrm{Au}$ & Sphere & 4.0 & - & 1.0 & $75 \%$ & 0 & $4 \%$ & Galactose & 100 & - & - & 10 \\
\hline $\mathrm{Au}$ & Sphere & 4.0 & - & 1.0 & $75 \%$ & 0 & $4 \%$ & Biotin & 100 & - & - & 10 \\
\hline $\mathrm{Au}$ & Sphere & 4.0 & - & - & $75 \%$ & 0 & $2 \%$ & Amino-MQAE + CV & $50-100$ & $9.6 \pm 0.6$ & - & 68 \\
\hline $\mathrm{Au}$ & Sphere & 4.0 & - & - & $75 \%$ & 0 & $2 \%$ & $\begin{array}{c}\mathrm{NH}_{2}-\mathrm{PEG}-\mathrm{Amino}- \\
\mathrm{MQAE}+\mathrm{CV}\end{array}$ & $50-100$ & $10.6 \pm 0.6$ & - & 68 \\
\hline $\mathrm{Au}$ & Sphere & 4.0 & - & - & $75 \%$ & 0 & $2 \%$ & $\begin{array}{c}\mathrm{NH}_{2}-\mathrm{PEG}-\mathrm{Amino}- \\
\mathrm{MQAE}+\mathrm{CV}\end{array}$ & $50-100$ & $11.0 \pm 0.6$ & - & 68 \\
\hline $\mathrm{Au}$ & Sphere & 4.0 & - & - & $75 \%$ & 0 & $2 \%$ & $\begin{array}{c}\mathrm{NH}_{2} \text {-PEG-Amino- } \\
\text { MQAE + CV }\end{array}$ & $50-100$ & $13.2 \pm 0.6$ & - & 68 \\
\hline $\mathrm{Au}$ & Sphere & 4.0 & - & 1.0 & $75 \%$ & 0 & $4 \%$ & Cresyl violet & 100 & - & - & 69 \\
\hline $\mathrm{Au}$ & Sphere & 4.0 & - & 1.2 & $75 \%$ & 0 & 0 & - & 100 & 13.2 & - & 70 \\
\hline $\mathrm{Au}$ & Sphere & 4.0 & - & 1.2 & $75 \%$ & 0 & 0 & - & 100 & 24.6 & - & 70 \\
\hline $\begin{array}{l}{ }^{198} \mathrm{Au} \\
\text { (neutron } \\
\text { irradiated }\end{array}$ & Sphere & $4.2 \pm 0.5$ & - & 1.1 & $75 \%$ & 0 & $2 \%$ & ATT0590 & 100 & - & - & 64 \\
\hline
\end{tabular}




\begin{tabular}{|c|c|c|c|c|c|c|c|c|c|c|c|c|}
\hline 1 & & & & & & & & & & & & \\
\hline $\begin{array}{c}{ }^{198} \mathrm{Au} \\
\text { (neutron } \\
\text { irradiated } \\
\text { ) }\end{array}$ & Sphere & $4.2 \pm 0.5$ & - & 1.1 & $75 \%$ & 0 & $3 \%$ & $\mathrm{ABz}-\mathrm{DOTA}+\mathrm{Gd}^{3+}$ & 100 & - & - & 64 \\
\hline $\begin{array}{c}{ }^{198} \mathrm{Au} \\
\text { (neutron } \\
\text { irradiated } \\
\text { ) }\end{array}$ & Sphere & $4.2 \pm 0.5$ & - & 1.1 & $75 \%$ & 0 & $3 \%$ & ABz-DOTA $+{ }^{111} \ln ^{3+}$ & 100 & - & - & 64 \\
\hline $\mathrm{Au}$ & Sphere & 4.6 & - & 3.25 & $100 \%$ & $10 \%$ & 0 & - & 100 & 11.7 & - & 71 \\
\hline $\mathrm{Au}$ & Sphere & 4.6 & - & 6.9 & $100 \%$ & $10 \%$ & 0 & - & 100 & - & - & 71 \\
\hline $\mathrm{Au}$ & Sphere & 4.6 & - & 9.95 & $100 \%$ & $10 \%$ & 0 & - & 100 & - & - & 71 \\
\hline $\mathrm{Au}$ & Sphere & 4.6 & - & 12.65 & $100 \%$ & $10 \%$ & 0 & - & 100 & - & - & 71 \\
\hline $\mathrm{Au}$ & Sphere & 4.6 & - & $\begin{array}{c}8.5 \\
21.6\end{array}$ & $100 \%$ & $10 \%$ & 0 & - & 100 & - & - & 71 \\
\hline $\mathrm{Au}$ & Sphere & 4.6 & - & 12.35 & $100 \%$ & $10 \%$ & 0 & - & 100 & - & - & 71 \\
\hline $\mathrm{Au}$ & Sphere & 4.6 & - & 11.55 & $100 \%$ & $10 \%$ & 0 & - & 100 & - & - & 71 \\
\hline $\mathrm{Au}$ & Sphere & 4.6 & - & 17.8 & $100 \%$ & $10 \%$ & 0 & - & 100 & - & - & 71 \\
\hline $\mathrm{Au}$ & Sphere & 4.6 & - & 3.95 & $100 \%$ & $10 \%$ & 0 & - & 100 & 13.5 & - & 71 \\
\hline $\mathrm{Au}$ & Sphere & 4.6 & - & 5.95 & $100 \%$ & $10 \%$ & 0 & - & 100 & 15.6 & - & 71 \\
\hline $\mathrm{Au}$ & Sphere & 4.6 & - & 8.2 & $100 \%$ & $10 \%$ & 0 & - & 100 & 17.9 & - & 71 \\
\hline $\mathrm{Au}$ & Sphere & 4.6 & - & 12.1 & $100 \%$ & $10 \%$ & 0 & - & 100 & 18.5 & - & 71 \\
\hline $\mathrm{Au}$ & Sphere & 4.6 & - & 15.1 & $100 \%$ & $10 \%$ & 0 & - & 100 & 18.6 & - & 71 \\
\hline $\mathrm{Au}$ & Sphere & 4.6 & - & 2.2 & $100 \%$ & $10 \%$ & - & - & 100 & - & - & 72 \\
\hline $\mathrm{Au}$ & Sphere & $4.6 \pm 1.0$ & - & 1.0 & $75 \%$ & 0 & - & - & 100 & $19 \pm 1$ & $-(55 \pm 6)$ & 73 \\
\hline $\mathrm{Au}$ & Sphere & $4.6 \pm 1.0$ & - & 1.0 & $75 \%$ & 0 & $2 \%$ & Cresyl violet + PEG & 100 & $40 \pm 5$ & $-(10 \pm 5)$ & 73 \\
\hline $\mathrm{Au}$ & Sphere & $4.6 \pm 1.0$ & - & 1.0 & $75 \%$ & 0 & $2 \%$ & $\begin{array}{c}\text { Cresyl violet + PEG- } \\
\text { CPP1 }\end{array}$ & 100 & $60 \pm 7$ & $-(11 \pm 5)$ & 73 \\
\hline $\mathrm{Au}$ & Sphere & $4.6 \pm 1.0$ & - & 1.0 & $75 \%$ & 0 & $2 \%$ & Cresyl violet + PEG- & 100 & $35 \pm 5$ & $-(13 \pm 4)$ & 73 \\
\hline
\end{tabular}




\begin{tabular}{|c|c|c|c|c|c|c|c|c|c|c|c|c|}
\hline & & & & & & & & CPP2 & & & & \\
\hline $\mathrm{Au}$ & Sphere & $4.7 \pm 0.8$ & - & - & $75 \%$ & 0 & 0 & - & - & - & - & 74 \\
\hline $\mathrm{Au}$ & Sphere & $4.8 \pm 0.7$ & - & 1.2 & $75 \%$ & 0 & $2 \%$ & ATTO590 & 50 & - & - & 77 \\
\hline $\mathrm{Au}$ & Sphere & 5 & - & - & $100 \%$ & 0 & 0 & - & - & $21 \pm 3$ & $-(19.1 \pm 0.4)$ & 53 \\
\hline $\mathrm{Au}$ & Sphere & 5 & - & - & $100 \%$ & 0 & 0 & - & - & $31.0 \pm 0.2$ & $-(10.1 \pm 0.6)$ & 53 \\
\hline $\mathrm{Au}$ & Sphere & 6.0 & - & - & $75 \%$ & 0 & 0 & - & 100 & - & - & 10 \\
\hline $\mathrm{Au}$ & Rod & $11 \pm 2$ & $\begin{array}{c}77.3 \pm \\
12.8\end{array}$ & - & $75 \%$ & 0 & 0 & - & 3000 & $29.6 \pm 0.5$ & $-(33.5 \pm 0.3)$ & 56 \\
\hline $\mathrm{Au}$ & Sphere & $24 \pm 3$ & - & - & $75 \%$ & 0 & 0 & - & 3000 & $27.0 \pm 0.2$ & $-(25.7 \pm 0.5)$ & 56 \\
\hline $\mathrm{Au}$ & Rod & $24 \pm 5$ & $\begin{array}{c}92.3 \pm \\
14.8\end{array}$ & - & $75 \%$ & 0 & 0 & - & 3000 & $9.0 \pm 0.2$ & $-(29.2 \pm 0.4)$ & 56 \\
\hline $\mathrm{Au}$ & Sphere & $50 \pm 7$ & - & - & $75 \%$ & 0 & 0 & - & 3000 & $43 \pm 1$ & $-(34 \pm 2)$ & 56 \\
\hline $\mathrm{Au}$ & Sphere & $60 \pm 8$ & - & - & $75 \%$ & 0 & 0 & - & 3000 & $47 \pm 1$ & $-(28.4 \pm 0.3)$ & 56 \\
\hline $\mathrm{Ag}$ & Sphere & $4.2 \pm 0.4$ & - & 1.0 & $75 \%$ & 0 & 0 & - & 100 & $12 \pm 3$ & $-(31 \pm 1)$ & 61 \\
\hline $\mathrm{Ag}$ & Sphere & $4.2 \pm 0.4$ & - & 1.0 & $75 \%$ & 0 & 0 & - & 100 & $13 \pm 4$ & $-(41 \pm 1)$ & 61 \\
\hline $\mathrm{Ag}$ & Sphere & $4.2 \pm 0.4$ & - & 1.0 & $75 \%$ & 0 & 0 & - & 100 & $12 \pm 3$ & $-(10.9 \pm 0.4)$ & 61 \\
\hline CdSe & Sphere & 2.6 & - & 1.2 & $75 \%$ & 0 & $0.1-5 \%$ & ATT0590 & 50 & - & - & 76 \\
\hline CdSe & Sphere & 4.7 & - & - & $100 \%$ & $10 \%$ & 0 & - & 100 & - & - & 71 \\
\hline CdSe/ZnS & Sphere & - & - & - & $100 \%$ & $10 \%$ & 0 & - & - & $5.5 \pm 1.3$ & - & 77 \\
\hline $\mathrm{CdSe} / \mathrm{ZnS}$ & Sphere & - & - & - & $100 \%$ & $10 \%$ & 0 & - & - & $10.8 \pm 1.4$ & - & 77 \\
\hline $\begin{array}{c}\text { CdSe/ZnS } \\
\text { (Evindent } \\
\text { QD490) }\end{array}$ & Sphere & & - & - & $100 \%$ & 0 & 0 & - & $100-200$ & 16.9 & - & 65 \\
\hline $\begin{array}{c}\text { CdSe/ZnS } \\
\text { (Evident } \\
\text { QD610) }\end{array}$ & Sphere & & - & - & $100 \%$ & 0 & 0 & - & $100-200$ & 23.6 & - & 65 \\
\hline $\mathrm{CdSe} / \mathrm{ZnS}$ & Sphere & & - & - & $75 \%$ & 0 & 0 & - & - & - & - & 74 \\
\hline $\mathrm{CdSe} / \mathrm{ZnS}$ & Sphere & & - & - & $75 \%$ & 0 & 0 & - & - & - & - & 74 \\
\hline
\end{tabular}




\begin{tabular}{|c|c|c|c|c|c|c|c|c|c|c|c|c|}
\hline $\mathrm{CdSe} / \mathrm{ZnS}$ & Sphere & 2.1 & - & 1.2 & $75 \%$ & 0 & $2 \%$ & ATT0590 & 100 & - & - & 75 \\
\hline $\mathrm{CdSe} / \mathrm{ZnS}$ & Sphere & 2.35 & - & 1.7 & $75 \%$ & 0 & 0 & - & 100 & - & - & 10 \\
\hline $\mathrm{CdSe} / \mathrm{ZnS}$ & Sphere & 2.56 & - & - & $75 \%$ & 0 & $2 \%$ & ATT0590 & 100 & - & - & 64 \\
\hline $\mathrm{CdSe} / \mathrm{ZnS}$ & Sphere & 2.56 & - & - & $75 \%$ & 0 & $3 \%$ & $\mathrm{ABz}-\mathrm{DOTA}+\mathrm{Gd}^{3+}$ & 100 & - & - & 64 \\
\hline $\mathrm{CdSe} / \mathrm{ZnS}$ & Sphere & 2.56 & - & - & $75 \%$ & 0 & $3 \%$ & ABz-DOTA $+{ }^{111} \ln ^{3+}$ & 100 & - & - & 64 \\
\hline $\mathrm{CdSe} / \mathrm{ZnS}$ & Sphere & $2.7 \pm 0.4$ & - & 1.2 & $75 \%$ & 0 & $\begin{array}{c}2 \text { to } \\
0.06 \%\end{array}$ & ATT05950 & 100 & - & - & 67 \\
\hline $\mathrm{CdSe} / \mathrm{ZnS}$ & Sphere & 2.8 & - & 1.1 & $75 \%$ & 0 & $0.1-5 \%$ & ATT0590 & 50 & - & - & 76 \\
\hline $\begin{array}{c}\text { CdSe/ZnS } \\
\text { (Evidot }^{\circledR} \\
490)\end{array}$ & Sphere & 3.2 & - & - & $75 \%$ & 0 & 0 & - & & 10 & - & 78 \\
\hline $\begin{array}{c}\text { CdSe/ZnS } \\
{\text { (Evidot }{ }^{\circledR}} \\
520)\end{array}$ & Sphere & 3.3 & - & - & $75 \%$ & 0 & 0 & - & - & 12 & - & 78 \\
\hline $\begin{array}{c}\text { CdSe/ZnS } \\
\text { (Evidot }^{\circledR} \\
540)\end{array}$ & Sphere & 3.4 & - & - & $75 \%$ & 0 & 0 & - & - & 12.4 & - & 78 \\
\hline $\begin{array}{c}\text { CdSe/ZnS } \\
\text { (Evidot }^{\circledR} \\
580 \text { ) }\end{array}$ & Sphere & 4.4 & - & - & $75 \%$ & 0 & 0 & - & - & 13.4 & - & 78 \\
\hline CdSe & Sphere & 4.7 & - & - & $100 \%$ & $10 \%$ & 0 & - & 100 & - & - & 71 \\
\hline $\mathrm{CdSe} / \mathrm{ZnS}$ & Sphere & 5.3 & - & 7.6 & $100 \%$ & $10 \%$ & 0 & - & 100 & 13.3 & - & 71 \\
\hline $\mathrm{CdSe} / \mathrm{ZnS}$ & Sphere & 5.3 & - & 8.75 & $100 \%$ & $10 \%$ & 0 & - & 100 & - & - & 71 \\
\hline $\mathrm{CdSe} / \mathrm{ZnS}$ & Sphere & 5.3 & - & 9.55 & $100 \%$ & $10 \%$ & 0 & - & 100 & - & - & 71 \\
\hline $\mathrm{CdSe} / \mathrm{ZnS}$ & Sphere & 5.3 & - & 10.3 & $100 \%$ & $10 \%$ & 0 & - & 100 & - & - & 71 \\
\hline $\mathrm{CdSe} / \mathrm{ZnS}$ & Sphere & 5.3 & - & 13.75 & $100 \%$ & $10 \%$ & 0 & - & 100 & - & - & 71 \\
\hline $\mathrm{CdSe} / \mathrm{ZnS}$ & Sphere & 5.3 & - & 10.25 & $100 \%$ & $10 \%$ & 0 & - & 100 & - & - & 71 \\
\hline $\mathrm{CdSe} / \mathrm{ZnS}$ & Sphere & 5.3 & - & 10.65 & $100 \%$ & $10 \%$ & 0 & - & 100 & - & - & 71 \\
\hline $\mathrm{CdSe} / \mathrm{ZnS}$ & Sphere & 5.3 & - & 10.5 & $100 \%$ & $10 \%$ & 0 & - & 100 & - & - & 71 \\
\hline
\end{tabular}




\begin{tabular}{|c|c|c|c|c|c|c|c|c|c|c|c|c|}
\hline $\mathrm{CdSe} / \mathrm{ZnS}$ & Sphere & 5.3 & - & 11.95 & $100 \%$ & $10 \%$ & 0 & - & 100 & - & - & 71 \\
\hline $\mathrm{CdSe} / \mathrm{ZnS}$ & Sphere & 5.3 & - & 10.05 & $100 \%$ & $10 \%$ & 0 & - & 100 & 16.8 & - & 71 \\
\hline $\mathrm{CdSe} / \mathrm{ZnS}$ & Sphere & 5.3 & - & 10.15 & $100 \%$ & $10 \%$ & 0 & - & 100 & 17.7 & - & 71 \\
\hline $\mathrm{CdSe} / \mathrm{ZnS}$ & Sphere & 5.3 & - & 11.15 & $100 \%$ & $10 \%$ & 0 & - & 100 & 18 & - & 71 \\
\hline $\mathrm{CdSe} / \mathrm{ZnS}$ & Sphere & 5.3 & - & 12.45 & $100 \%$ & $10 \%$ & 0 & - & 100 & 18.2 & - & 71 \\
\hline $\mathrm{CdSe} / \mathrm{ZnS}$ & Sphere & 5.3 & - & 14.45 & $100 \%$ & $10 \%$ & 0 & - & 100 & 18.4 & - & 71 \\
\hline $\mathrm{CdSe} / \mathrm{ZnS}$ & Sphere & 5.3 & - & - & $75 \%$ & $5 \%$ & $1 \%$ & АTT0590 & 70 & - & - & 79 \\
\hline $\mathrm{CdSe} / \mathrm{ZnS}$ & Sphere & 5.3 & - & - & $75 \%$ & $5 \%$ & 0 & - & 70 & - & - & 79 \\
\hline $\mathrm{CdSe} / \mathrm{ZnS}$ & Sphere & 5.3 & - & - & $75 \%$ & 0 & $5 \%$ & ATT0590 & 70 & - & - & 69 \\
\hline $\mathrm{CdSe} / \mathrm{ZnS}$ & Sphere & 7.0 & - & 1.0 & $100 \%$ & $6 \%$ & 0 & - & $\begin{array}{l}10,15 \\
20,60\end{array}$ & $\begin{array}{l}19 \pm 2 \\
24 \pm 2\end{array}$ & - & 63 \\
\hline $\mathrm{CdSe} / \mathrm{ZnS}$ & Sphere & 7.0 & - & 1.0 & $100 \%$ & $10 \%$ & 0 & - & 100 & $\begin{array}{l}19 \pm 2 \\
24 \pm 2\end{array}$ & - & 63 \\
\hline $\mathrm{CdSe} / \mathrm{ZnS}$ & Sphere & 7.0 & - & - & $100 \%$ & $10 \%$ & 0 & - & 100 & - & - & 17 \\
\hline CdTe & Sphere & 6.3 & - & 2.8 & $100 \%$ & $10 \%$ & 0 & - & & - & - & 71 \\
\hline $\mathrm{CoPt}_{3}$ & Sphere & 8.0 & - & 1.0 & $100 \%$ & $10 \%$ & 0 & - & 100 & - & - & 63 \\
\hline $\mathrm{Fe}_{3} \mathrm{O}_{4}$ & Sphere & 8 & - & - & $75 \%$ & 0 & 0 & - & - & $145 \pm 2$ & - & 80 \\
\hline $\mathrm{Fe}_{3} \mathrm{O}_{4}$ & Sphere & 8 & - & - & $75 \%$ & 0 & 0 & - & - & $178 \pm 7$ & - & 80 \\
\hline $\mathrm{Fe}_{3} \mathrm{O}_{4}$ & Sphere & $8.0 \pm 1.1$ & - & 1.0 & $75 \%$ & 0 & $0.75 \%$ & DY-636 & 100 & $6.2 \pm 0.2$ & $-(55 \pm 3)$ & 66 \\
\hline $\mathrm{Fe}_{2} \mathrm{O}_{3}$ & Sphere & 9.2 & - & 1.0 & $100 \%$ & $10 \%$ & 0 & - & 100,519 & - & - & $\begin{array}{c}\text { Pelle } \\
\text { grino } \\
\# 911 \\
7\}\end{array}$ \\
\hline $\mathrm{Fe}_{2} \mathrm{O}_{3}$ & Sphere & 9.2 & - & 1.0 & $100 \%$ & $13 \%$ & 0 & - & $\begin{array}{c}93,182 \\
260\end{array}$ & - & - & 63 \\
\hline $\mathrm{Fe}_{2} \mathrm{O}_{3}$ & Sphere & $10.8 \pm 0.5$ & - & 1.2 & $75 \%$ & 0 & $2 \%$ & ATT0590 & 50 & - & - & 64 \\
\hline $\mathrm{Fe}_{2} \mathrm{O}_{3}$ & Sphere & $10.8 \pm 0.5$ & - & 1.2 & $75 \%$ & 0 & $3 \%$ & $\mathrm{ABz}-\mathrm{DOTA}+\mathrm{Gd}^{3+}$ & 50 & - & - & 64 \\
\hline $\mathrm{Fe}_{2} \mathrm{O}_{3}$ & Sphere & $10.8 \pm 0.5$ & - & 1.2 & $75 \%$ & 0 & $3 \%$ & $\mathrm{ABz}-\mathrm{DOTA}+{ }^{111} \ln ^{3+}$ & 50 & - & - & 64 \\
\hline
\end{tabular}




\begin{tabular}{|c|c|c|c|c|c|c|c|c|c|c|c|c|}
\hline $\mathrm{Fe}_{2} \mathrm{O}_{3}$ & Sphere & $10.8 \pm 0.12$ & - & - & $100 \%$ & 0 & 0 & - & $100-200$ & - & - & 65 \\
\hline $\mathrm{Fe}_{2} \mathrm{O}_{3}$ & Sphere & 13.0 & - & 1.0 & $75 \%$ & 0 & 0 & - & 100 & - & - & 10 \\
\hline FePt & Sphere & & - & - & $75 \%$ & 0 & $2 \%$ & DY-636 & 300 & - & - & 81 \\
\hline FePt & Sphere & $3.1 \pm 0.4$ & - & 1.0 & $75 \%$ & 0 & $0.75 \%$ & DY-636 & 100 & $4.2 \pm 0.2$ & $-(44 \pm 3)$ & 66 \\
\hline FePt & Sphere & $3.1 \pm 0.4$ & - & 1.0 & $75 \%$ & 0 & $0.75 \%$ & DY-636 + Glucose & 100 & $4.6 \pm 0.6$ & $-(25 \pm 3)$ & 66 \\
\hline FePt & Sphere & $3.1 \pm 0.4$ & - & 1.0 & $75 \%$ & 0 & $0.75 \%$ & $\begin{array}{c}\text { DY-636 + PEG (750 } \\
\text { Da) }\end{array}$ & 100 & $4.8 \pm 0.5$ & $-(17.8 \pm 0.5)$ & 66 \\
\hline $\mathrm{FePt}$ & Sphere & $3.1 \pm 0.4$ & - & 1.0 & $75 \%$ & 0 & $0.75 \%$ & $\begin{array}{c}\text { DY-636 + PEG (5 } \\
\text { kDa) }\end{array}$ & 100 & $6.5 \pm 0.7$ & $-(28 \pm 1)$ & 66 \\
\hline FePt & Sphere & $3.1 \pm 0.4$ & - & 1.0 & $75 \%$ & 0 & $0.75 \%$ & $\begin{array}{c}\text { DY-636 + PEG (10 } \\
\text { kDa) }\end{array}$ & 100 & $10.6 \pm 0.9$ & $-(14.3 \pm 0.4)$ & 66 \\
\hline FePt & Sphere & $3.1 \pm 0.4$ & - & 1.0 & $75 \%$ & 0 & $0.75 \%$ & DY-636 & 100 & $4.2 \pm 0.2$ & $-(44 \pm 3)$ & 66 \\
\hline FePt & Sphere & $3.2 \pm 0.4$ & - & 1.1 & $75 \%$ & 0 & $0-2 \%$ & ATT0590 & 200 & 11.2 & - & 82 \\
\hline FePt & Sphere & $3.5 \pm 0.6$ & - & - & $75 \%$ & 0 & 0 & - & - & $9 \pm 5$ & $-(66 \pm 2)$ & 83 \\
\hline $\mathrm{MnFe}_{2} \mathrm{O}_{3}$ & Sphere & 4.5 & - & 1.0 & $100 \%$ & $10 \%$ & 0 & - & 100 & - & - & 84 \\
\hline $\mathrm{MnFe}_{2} \mathrm{O}_{3}$ & Sphere & 6.0 & - & 1.0 & $100 \%$ & $10 \%$ & 0 & - & 100 & - & - & 84 \\
\hline $\mathrm{MnFe}_{2} \mathrm{O}_{3}$ & Sphere & 7.5 & - & 1.0 & $100 \%$ & $10 \%$ & 0 & - & 100 & - & - & 84 \\
\hline $\mathrm{ZnO}$ & Sphere & $6.9 \pm 1.5$ & - & 1.1 & $75 \%$ & 0 & - & - & 100 & $18 \pm 2$ & $-(35 \pm 4)$ & 85 \\
\hline
\end{tabular}

Table VI.3.2. List of parameters used for overcoating various NPs with PMA-g-dodecyl or functionalized PMA-g-dodecyl polymers. $R_{\text {chain/mon }}$ describes the percentage of anhydride rings of PMA that has been used for linking hydrophobic side chains (dodecyl chains). Note, that in some references a slightly different polymer was used, in which there is one hydrophobic side chain in addition to each anhydride ring, and thus $R_{\text {chain } / \text { mon }}=100 \%{ }^{17,63,71,77,79,84}$ and thus $R_{\text {chain } / \text { mon }}+R_{\text {cross } / \text { mon }}+R_{\text {func/mon }}>100 \%$. $R_{\text {cross } / \text { mon describes the percentage of anhydride rings }}$ that have been used for addition of a crosslinker ${ }^{63}$. $R_{\text {func/mon }}$ describes the percentage of anhydride rings that have been used to add organic functionalities such as fluorophores. The following fluorophores have been integrated into PMA, as given together with their excitation wavelength and the extinction coefficient at their excitation wavelength: i) ATTO590 $\left(\lambda_{\text {ext }}=590 \mathrm{~nm}, \varepsilon=1.2 \times 10^{5} M^{-1} \mathrm{~cm}^{-1}\right)$, ii) DY-636 $\left(\lambda_{\text {ext }}=\right.$ $\left.645 \mathrm{~nm}, \varepsilon=1.9 \times 10^{5} \mathrm{M}^{-1} \mathrm{~cm}^{-1}\right)$, ii) SNARF $\left(\lambda_{\text {ext }}=514 \mathrm{~nm}, \varepsilon=\right.$ depends on $\left.\mathrm{pH}\right)$, iv) Amino-MQAE $\left.\left(\lambda_{\text {ext }}=350 \mathrm{~nm}, \varepsilon=2.8 \times 10^{3} \mathrm{M}^{-1} \mathrm{~cm}^{-1}\right), \mathrm{v}\right) \mathrm{Cresyl}$ 
Violet $(\mathrm{CV})\left(\lambda_{\text {ext }}=590 \mathrm{~nm}\right)$, vi) Oregon Green $\left(\lambda_{\text {ext }}=494 \mathrm{~nm}, \varepsilon=7.02 \cdot 10^{4} \mathrm{M}^{-1} \mathrm{~cm}^{-1}\right)$, vii) 3-hydroxyflavone dye $(F E)\left(\lambda_{\text {ext }}=410 \mathrm{~nm}\right)$, viii) Fluoresceinamine $\left(\lambda_{\text {ext }}=515 \mathrm{~nm}\right)$. Hydrodynamic diameter $\left(d_{h}\right)$ and zeta-potential $\zeta$ values are provided $(c f . \xi X)$.

\begin{tabular}{|c|c|c|c|c|c|c|c|}
\hline $\begin{array}{l}\text { NP core } \\
\text { material }\end{array}$ & $\mathrm{d}_{\mathrm{c}}[\mathrm{nm}]$ & Polymer & $\begin{array}{c}\mathrm{M}_{\mathrm{w}} \text { (polyme } \\
\mathrm{r}) \\
{[\mathrm{g} / \mathrm{mol}]}\end{array}$ & $\begin{array}{l}\mathrm{R}_{\mathrm{P} / \mathrm{Area}} \\
{\left[\mathrm{nm}^{-2}\right]}\end{array}$ & $\mathrm{d}_{\mathrm{h}}[\mathrm{nm}]$ & $\zeta[\mathrm{mV}]$ & Ref. \\
\hline $\mathrm{Au}$ & 4.0 & PEO & 600,000 & & & & 86 \\
\hline $\mathrm{Au}$ & 3.8 & $\begin{array}{l}\text { PDMAEMA-stat- } \\
\text { PLMA (11:89) }\end{array}$ & 9950 & $\geq 50$ & & & 87 \\
\hline $\mathrm{Au}$ & 3.8 & $\begin{array}{l}\text { PDMAEMA-stat- } \\
\text { PLMA (19:81) }\end{array}$ & 11000 & $\geq 50$ & & & 87 \\
\hline $\mathrm{Au}$ & 3.8 & $\begin{array}{l}\text { PDMAEMA-stat- } \\
\text { PLMA }(20: 80)\end{array}$ & 8650 & $\geq 50$ & & & 87 \\
\hline $\mathrm{Au}$ & 3.8 & $\begin{array}{c}\text { PDMAEMA-stat- } \\
\text { PLMA (38:62) }\end{array}$ & 9500 & $\geq 50$ & $22 \pm 13$ & $+(28 \pm 15)$ & 87 \\
\hline $\mathrm{Au}$ & 3.8 & $\begin{array}{l}\text { PDMAEMA-stat- } \\
\text { PLMA (47:53) }\end{array}$ & 46200 & $\geq 50$ & & & 87 \\
\hline $\mathrm{Au}$ & 3.8 & $\begin{array}{l}\text { PDMAEMA-stat- } \\
\text { PLMA (51:49) }\end{array}$ & 47000 & $\geq 50$ & & & 87 \\
\hline $\mathrm{Au}$ & 3.8 & $\begin{array}{l}\text { PDMAEMA-stat- } \\
\text { PLMA }(53: 47)\end{array}$ & 11000 & $\geq 50$ & $7 \pm 2$ & $+(30 \pm 14)$ & 87 \\
\hline $\mathrm{Au}$ & 3.8 & $\begin{array}{l}\text { PDMAEMA-stat- } \\
\text { PLMA (64:36) }\end{array}$ & 12000 & $\geq 50$ & & & 87 \\
\hline $\mathrm{Au}$ & 3.8 & $\begin{array}{l}\text { PDMAEMA-stat- } \\
\text { PLMA (73:27) }\end{array}$ & 6500 & $\geq 50$ & & & 87 \\
\hline $\mathrm{Au}$ & 3.8 & $\begin{array}{l}\text { PDMAEMA-stat- } \\
\text { PLMA }(75: 25)\end{array}$ & 46000 & $\geq 50$ & & & 87 \\
\hline $\mathrm{Au}$ & 3.8 & $\begin{array}{l}\text { PDMAEMA-stat- } \\
\text { PLMA }(76: 24) \\
\end{array}$ & 7000 & $\geq 50$ & $28 \pm 21$ & $+(31 \pm 6)$ & 87 \\
\hline $\mathrm{Au}$ & 3.8 & PMAPHOS(OMe & 10500 & $\geq 50$ & & & 87 \\
\hline
\end{tabular}




\begin{tabular}{|c|c|c|c|c|c|c|c|}
\hline & & $\begin{array}{c})_{2} \text {-stat-PLMA } \\
(21: 79)\end{array}$ & & & & & \\
\hline $\mathrm{Au}$ & 3.8 & $\begin{array}{c}\text { PMAPHOS(OMe } \\
\text { ) }_{2} \text {-stat-PLMA } \\
(25: 75)\end{array}$ & 8000 & $\geq 50$ & & & 87 \\
\hline $\mathrm{Au}$ & 3.8 & $\begin{array}{c}\text { PMAPHOS(OMe } \\
\text { )2-stat-PLMA } \\
(35: 65)\end{array}$ & 11800 & $\geq 50$ & $13 \pm 3$ & $-(25 \pm 5)$ & 87 \\
\hline $\mathrm{Au}$ & 3.8 & $\begin{array}{l}\text { PMAPHOS(OMe } \\
\text { ) }_{2} \text {-stat-PLMA } \\
(40: 60)\end{array}$ & 9500 & $\geq 50$ & $11 \pm 4$ & $-(21 \pm 9)$ & 87 \\
\hline $\mathrm{Au}$ & 3.8 & $\begin{array}{c}\text { PMA-g-D1 } \\
(68: 32)\end{array}$ & 6000 & $\geq 50$ & $10.6 \pm 0.7$ & $-(24 \pm 4)$ & 87 \\
\hline $\mathrm{CdSe} / \mathrm{ZnS}$ & 4 & $\begin{array}{l}\text { PDMAEMA-stat- } \\
\text { PLMA (38:62) }\end{array}$ & 9500 & 50 & & & 87 \\
\hline $\mathrm{CdSe} / \mathrm{ZnS}$ & 4 & $\begin{array}{l}\text { PDMAEMA-stat- } \\
\text { PLMA (53:47) }\end{array}$ & 11000 & 50 & & & 87 \\
\hline $\mathrm{CdSe} / \mathrm{ZnS}$ & 4 & $\begin{array}{l}\text { PDMAEMA-stat- } \\
\text { PLMA }(76: 24)\end{array}$ & 7000 & 50 & & & 87 \\
\hline $\mathrm{CdSe} / \mathrm{ZnS}$ & 4 & $\begin{array}{l}\text { PDMAEMA-stat- } \\
\text { PLMA }(35: 65)\end{array}$ & 11800 & 50 & & & 87 \\
\hline $\mathrm{CdSe} / \mathrm{ZnS}$ & 4 & $\begin{array}{l}\text { PDMAEMA-stat- } \\
\text { PLMA (40:60) }\end{array}$ & 9500 & 50 & & & 87 \\
\hline $\mathrm{CdSe} / \mathrm{ZnS}$ & 4 & $\begin{array}{l}\text { PDMAEMA-stat- } \\
\text { PLMA (68:32) }\end{array}$ & 6000 & 50 & & & 87 \\
\hline $\mathrm{Au}$ & $4.6 \pm 1.1$ & $\begin{array}{l}\text { PMAPHOS-stat- } \\
\text { PLMA }\end{array}$ & 9000 & 50 & $11 \pm 3$ & $-(40 \pm 10)$ & 88 \\
\hline $\mathrm{Au}$ & $4.6 \pm 1.1$ & $\begin{array}{l}\text { PTMAEMA-stat- } \\
\text { PLMA }\end{array}$ & 16300 & 50 & $13 \pm 3$ & $+(10 \pm 9)$ & 88 \\
\hline $\mathrm{Au}$ & $4.6 \pm 1.1$ & $\begin{array}{l}\text { PMAPHOS-stat- } \\
\text { PLMA-stat-PDI }\end{array}$ & 9500 & 50 & $13 \pm 6$ & $-(23 \pm 8)$ & 88 \\
\hline $\mathrm{Au}$ & $4.6 \pm 1.1$ & $\begin{array}{l}\text { PTMAEMA-stat- } \\
\text { PLMA-stat-PDI }\end{array}$ & 17800 & 50 & $7 \pm 2$ & $+(17 \pm 1)$ & 88 \\
\hline
\end{tabular}


Table VI.3.3. List of amphiphilic polymers others than PMA which have been used for the overcoating of hydrophobic NPs. For the polymer materials the ratio of charged (x) to uncharged $(y)$ monomer units in the polymer is noted as $(x: y)$. 


\section{Purification of hydrophilic NPs}

\section{VII.1 General considerations \\ VII.2 Filtration, ultrafiltration, and dialysis \\ VII.3 Size exclusion chromatography \\ VII.4 Gel electrophoresis \\ VII.5 Ultracentrifugation}

\section{VII.1 General considerations}

Generally, NPs should be purified from excess free reactants, byproducts and ligand molecules in the solution. The selection of a purification technique for a NP solution depends on many variables such as colloidal stability of NPs during purification, efficiency of the purification process, the desired purity level, size and nature of molecules/ligands to be removed, and availability of the respective purification techniques. Unfortunately, there is no universal purification tool for any generic NP solution, but indeed proper purification techniques should be carefully selected for each reaction, to ensure efficient separation between NPs (products) and free excess reactants (impurities). Purification on the other hand also may change the properties of the NPs, e.g. modify their ligand shell ${ }^{18}$.

As example, the polymer coating procedure in section VI.3 employs excess polymer in the reaction, which must be removed afterward (post synthesis purification). It is worth mentioning that avoiding excess addition of polymer may result in incomplete coating and thus induce NP aggregation. The free residual polymer in aqueous solution forms polymeric micelles, with a hydrophobic cavity and a hydrophilic surface, $c f$. Figure VII.1.1 ${ }^{79}$. They are stable assemblies of several polymer molecules, in which the hydrophobic tails point towards the inside of the micelle, and the hydrophilic backbones are exposed to the outside. Thus they can be thought as "empty" polymer shells without embedded inorganic NPs. The polymer micelles have the size of a few $\mathrm{nm}$, and thus it is hard to be separated from NPs with similar size using size exclusion chromatography. However, efficient separation could be achieved by other separation methods such as gel electrophoresis and ultracentrifugation (cf. §VII.5).

Purification is paramount for many applications. In case of cytotoxicity studies it is required to ensure that any effect is related to the NPs and not to residuals in solution. Also for quantitative labelling, empty micelles will compete with polymer coated NPs for binding to the same target, however, without any label (i.e. the NP core) inside, and thus without being detected. Note, that polymeric micelles are hard to detect, as 
the amphiphilic polymer itself does not have any specific absorption of light, and also is not fluorescent. In case the polymer has been modified with organic fluorophores these micelles can be detected by their fluorescence ${ }^{79}$. In addition negative staining of the polymer allows for visualizing empty polymer micelles with transmission electron microscopy, cf. §VII.5.
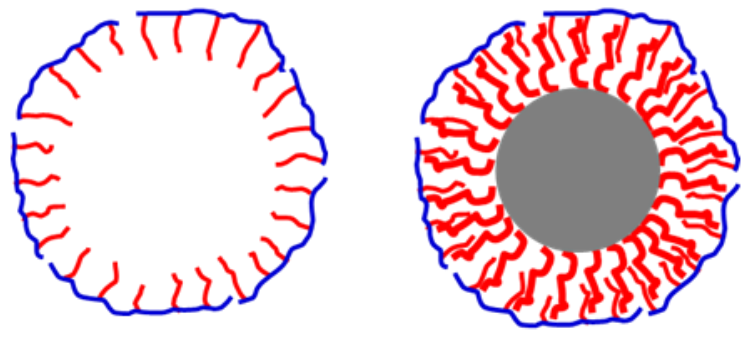

Figure VII.1.1. Empty polymer micelles (left) versus polymer-coated Au NPs (right). Due to similar size, separation using size exclusion chromatography is not efficient

\section{VII.2 Filtration, ultrafiltration, and dialysis}

The synthesis of NPs sysnthesis in many occasions is associated with the formation of large aggregates/agglomerates due to NP aggregation, which can be removed from solution by simple filtration via a syringe membrane filter $(0.22 \mu \mathrm{m}$ pore size, Carl Roth, \#KY62.1) as per Figure VII.1.2. In case the NP solution does not pass easily through the filter, addition of water to the NP solution, i.e. dilution, may help.
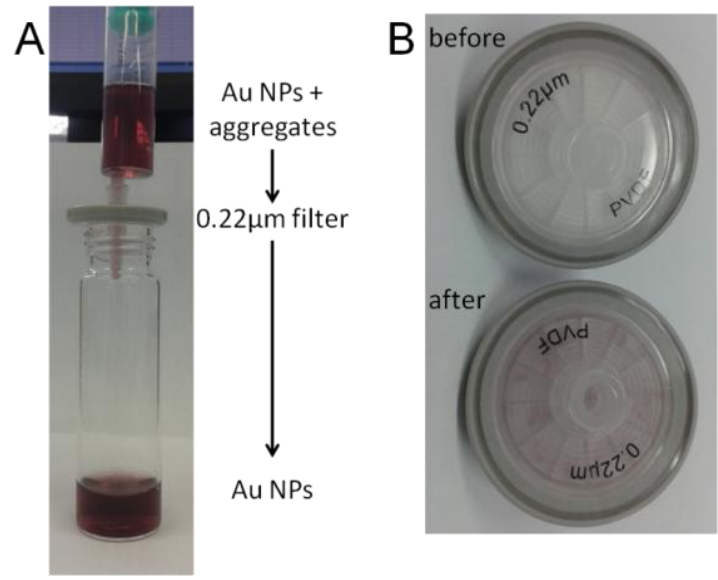

Figure VII.2.1. A) Purification of PMA-g-dodecyl capped Au NPs ( $\approx 4 \mathrm{~nm}$ core diameter,

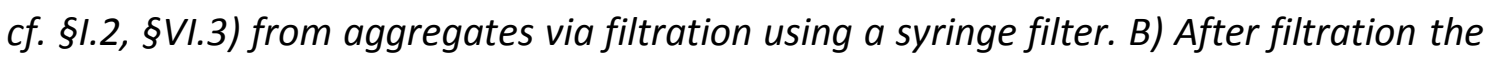
aggregates can be seen in the filter by naked eye. 
Ultrafiltration can be employed to purify and to concentrate NPs. Ultrafiltration also allows for exchange of the suspending media, for example from buffer to water or vice versa. Briefly, NP suspension is placed in the centrifuge filter (available from various suppliers; e.g. Vivaspin 20 from Sartorius or Amicon ${ }^{\circledR}$ Ultra $4 \mathrm{~mL}$ Centrifugal Filters), typically 100,000 Da molecular weight cutoff (MWCO). The pores of the filter are big enough to be passed by small molecules, but retain the NPs. Upon centrifugation the filtrate that contains free ligands/reactants is collected in the outer vial below the filter membrane, and the NP suspension is concentrated in the inner vial. Fresh solvent can be added to the NP concentrate and the filtration can be repeated for several rounds of clean up as required (Figure VII.2.2 and Figure VII.2.3). Note that excessive purification/concentration may induce NPs aggregation, which can also block the filter.

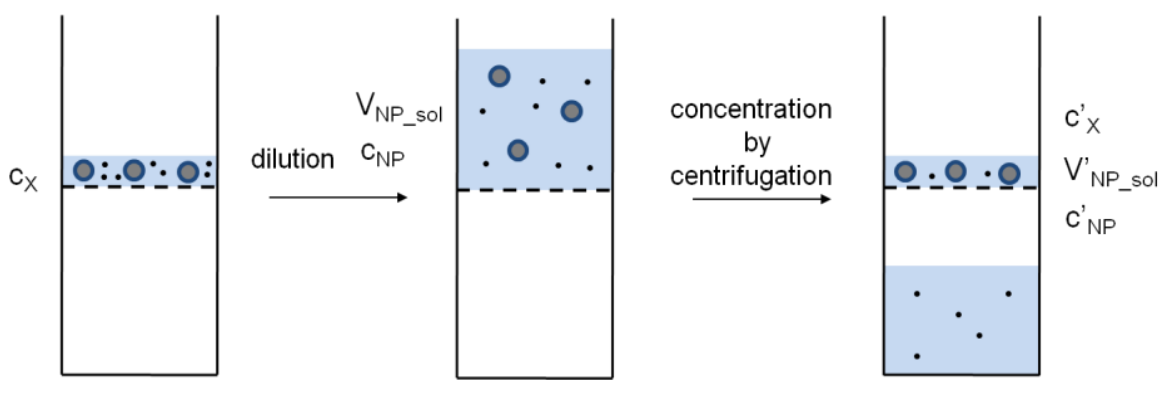

Figure VII.2.2. Ultrafiltration of hydrophilic NPs (drawn as gray spheres with blue surface) using a centrifugal filter. The NP suspension contains impurities (small black dots) of concentration $c_{x}$. First, the NP suspension is diluted with fresh solvent to a volume $\left(V_{N P_{-} s o l}\right)$, resulting in NP concentration of $c_{N P}$. Upon centrifugation only solvent and the small impurities pass the filter membrane, which results in an increase in NP concentration $\left(c^{\prime}{ }_{N P}=c_{N P} \cdot V^{\prime}{ }_{N P_{-} s o l} / V_{N P_{-} s o l}\right)$ and a decrease in impurities concentration $\left(c^{\prime}{ }_{X}\right.$ $\left.=c_{X} \cdot V_{N P_{-} s o} / V^{\prime}{ }_{N P_{-} s o l}\right)$.

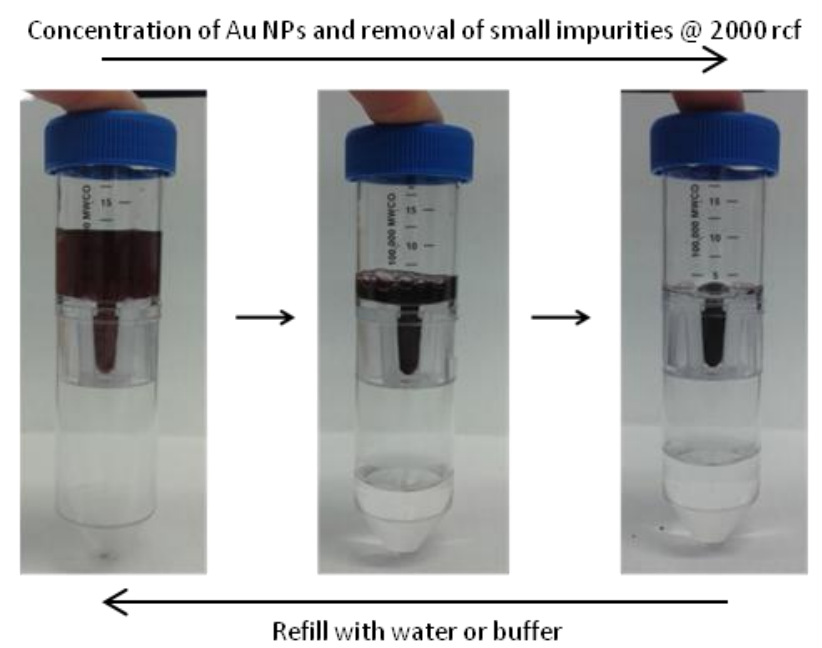


Figure VII.2.3. Ultrafiltration using a centrifugal filter to purify and concentrate Au NPS. (PES (Polyethersulfon)-membrane centrifugal filter with 100 kDa MWCO, Sartorius Stedim, \#VS2042)

Dialysis is a widely used method to purify NP suspension from free impurities. The method is described in Figure VII.2.4. The NP suspension is filled in a semi-permeable membrane dialysis bag with defined pore size and molecular weight cut-off (MWCO), which then is placed in a beaker filled with large volume of fresh buffer/solvent. Molecules smaller than the MWCO can penetrate the membrane and diffuse out from the dialysis bag to the bulk solution in the beaker. Dialysis is driven by the concentration gradient between the dialysis bag and the concentration in the beaker. Thus the process occurs until equilibrium is achieved. At this point, the bath needs to be replaced with fresh solvent. Dialysis is easy to perform, and in fact is a "gentle" treatment to the NPs, since no mechanical stress is placed on NPs (the case of centrifugation as an example). However, it is time consuming and it has lower efficiency, compared to ultrafiltration. In addition, due to osmotic pressure upon dialysis, the volume of the NP suspension in the dialysis bag may increase resulting in dilution of the NP suspension

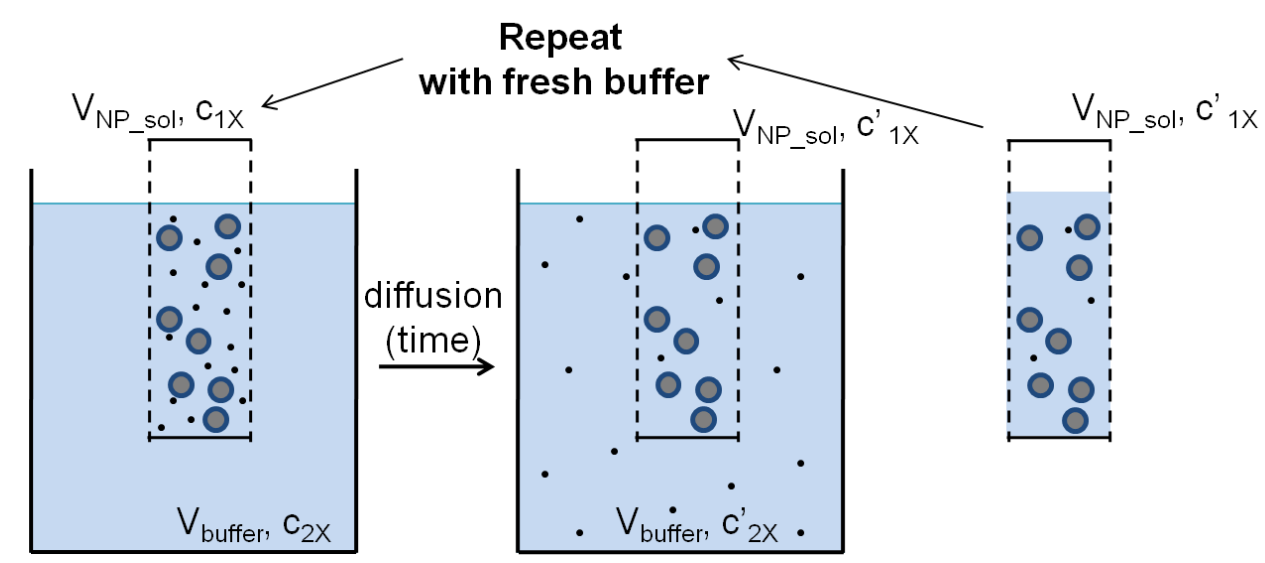

Figure VII.2.4. Dialysis of hydrophilic NPs (drawn as gray spheres with blue surface) using dialysis bag of volume $V_{N P_{-} s o l}$. Besides the NPs there are also small impurities of concentration $c_{1 x}$ in the NP suspension. The dialysis bag is placed into a bath solution of volume $V_{\text {buffer, }}$ in which there are no impurities, i.e. $c_{2 x}=0$. Upon diffusion impurities move from the dialysis bag into the bath. At equilibrium, concentration of impurities in the dialysis bag is reduced to $c^{\prime}{ }_{1 X}\left(c^{\prime}{ }_{1 X}=c_{1 X} \times V_{N P_{-} \text {sol }} /\left(V_{N P_{-} s o l}+V_{b u f f e r}\right)\right)$.

\section{VII.3 Size exclusion chromatography}


In size exclusion chromatography (SEC), a NP suspension is run through a porous gel matrix driven by gravity or by pressure. The separation mechanism is based on the interaction between the NPs and the pores of the gel: "small" NPs can enter the pores and are thus get retained, whereas "large" NPs are excluded from the pores and thus pass the gel faster without interaction. In this way "large" NPs are eluted first, and "small" NPs take longer to diffuse into and out of the pores, and thus are eluted later, cf. Figure VII.3.1. "Small" and "large" sizes of NP are relative to the size of the pores of the gel, i.e. the pore size has to be selected based on the size of NPs in the sample. The gel beads used to form the gel matrix have to be inert, i.e. the NPs must not react and/or stick to them. SEC is frequently used for desalting, i.e. small ions are retained, whereas the sample, such as proteins, are eluted ${ }^{89,90}$. In this way SEC can be also used to purify NPs from smaller excess molecules (e.g. salt or capping agent) in solution. For quantitative analysis, SEC columns need to be calibrated to estimate the hydrodynamic diameter $d_{h}$ of NPs ${ }^{71}$. An example of one elution profile is shown in Figure VII.3.2 and Figure VII.3.3.

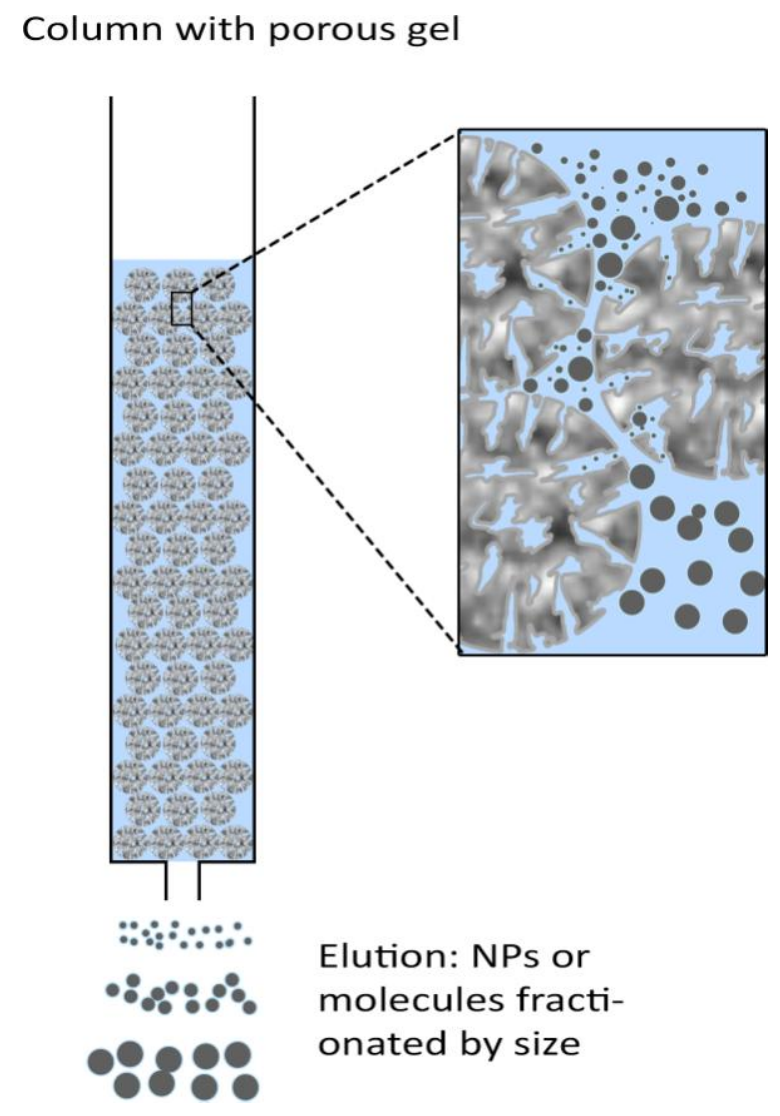

Figure VII.3.1. Principle of size exclusion chromatography. Small impurities enter the pores of gel beads and are thus retarded, whereas larger NPs are eluted faster. 
The possibility of assembling a SEC column in a high performance liquid chromatography (HPLC) system (cf. Figure VII.3.2) allows for an automatic sample injection and collection, a customizable constant pressure all over the sample run, and the possibility of multiple programmed detectors. Optimizing parameters related to the stationary phase, the column dimensions, the mobile phase or the process flow, allow fine-tuning of the separation quality. It is important to note that a pre-filtering step of NP suspension prior to sample injection is important.

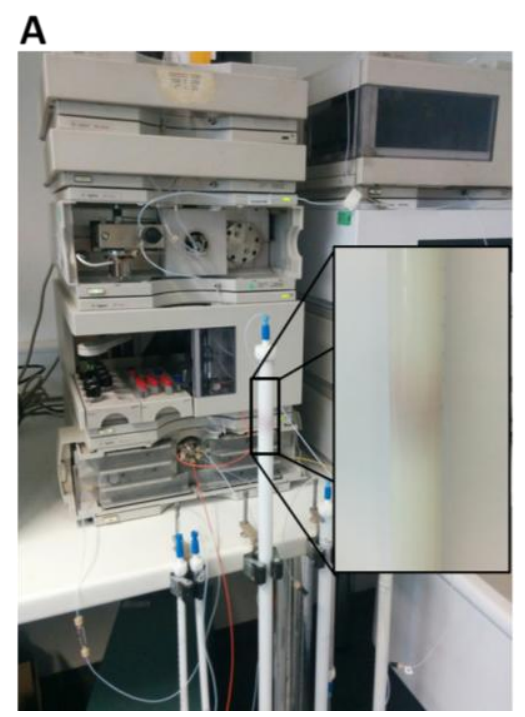

B

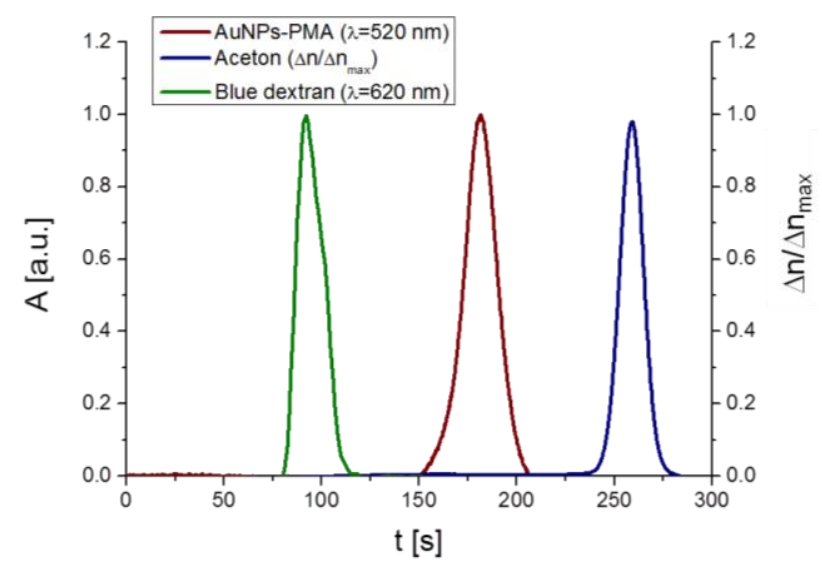

Figure VII.3.2. A) Elution of PMA-g-dodecyl-capped Au NPs ( $\approx 4 \mathrm{~nm}$ core diameter, cf. $\S I .2, \S V I .3)$ via high performance liquid chromatography (HPLC; Agilent 1100 Series) on a size exclusion chromatography column (75 cm length and $1.5 \mathrm{~cm}$ diameter) filled with Sephacryl S40OHR as stationary phase and sodium borate buffer $\mathrm{pH}=9$ as mobile phase. The flow rate was set as $0.5 \mathrm{~mL} \cdot \mathrm{min}^{-1}$. The inset shows the red band of eluted Au NPs. B) Size exclusion chromatograph of PMA-g-dodecyl-capped Au NPs $(\approx 4 \mathrm{~nm}$ diameter, cf. $\S I .2$, SVI.3) using similar conditions in A. The normalized absorption A at $520 \mathrm{~nm}$ is plotted versus the elution time of the Au NPs (dark red curve). For column calibration, peaks of blue dextran shown as normalized absorption at $620 \mathrm{~nm}$ (drawn in green) and acetone shown as normalized changes of the refractive index (blue) are also represented on the chromatogram as reference of the range of the column retention time at these working conditions. In the case of the acetone the changes of the refractive index are measured via an internal reference cell and a sample cell set in the instrument. Both are initially flushed with the mobile phase until the refractive index is the same in both cells, and at this moment the zero value is adjusted. Once the measurement starts the reference cell stays closed and the column eluate flows through the sample cell. The final signal is expressed as Refractive Index Units (RIU), being the difference $\Delta n$ between the refractive index in the sample cell and the refractive index in the reference cell. 


\section{VII.4 Gel electrophoresis}

Gel electrophoresis is based on the mobility of charged molecules in a gel matrix under applied electric field. The higher the charge of the molecules, the faster they migrate through the gel ${ }^{91}$. The bigger the molecules, the slower they can pass the pores in the gel and that the slower they migrate ${ }^{52}$. In this way, assuming similar surface charge density, "large" NPs run slower in gel than "small" NPs ${ }^{51}$. Separation of NPs with various particle sizes can be optimized by adjusting the concentration of the gel and thus the gel mean pore size ${ }^{51}$.

For gel preparation (here $2 \% \mathrm{w} / \mathrm{v}$ agarose), $3 \mathrm{~g}$ of agarose powder (Invitrogen, \#15510027, "Agarose UltraPure") were dissolved in $150 \mathrm{~mL}$ of $0.5 \times$ TBE buffer (44.5 $\mathrm{mM}$ Tris-borate and $1 \mathrm{mM}$ ethylenediaminetetraacetic acid (EDTA), $\mathrm{pH}=8.3$, SigmaAldrich, \#T3913) in a $500 \mathrm{~mL}$ Erlenmeyer flask. The solution was then covered with a watch-glass and heated in a microwave oven at $380 \mathrm{~W}$ for $8 \mathrm{~min}$ until the solution started to boil. The flask was swirled to help all agarose powder to dissolve, and was then heated again to the boiling point. The hot and clear agarose solution was poured in a $10 \times 15 \mathrm{~cm}$ gel tray leveled in a gel caster (BioRad). A comb (1, 2, 15, 20 or 30 wells) was placed into the gel, and the whole device was covered with an alumina or polyethylene foil. The gel was allowed to cool down to RT and to solidify, which typically takes $1 \mathrm{~h}, c f$. Figure VII.4.1. After solidification the comb was removed, the gel was taken out of the casting device and placed into the electrophoresis device (BioRad Subcell GT wide mini), which was filled with $0.5 \times$ TBE buffer until the whole gel was covered.
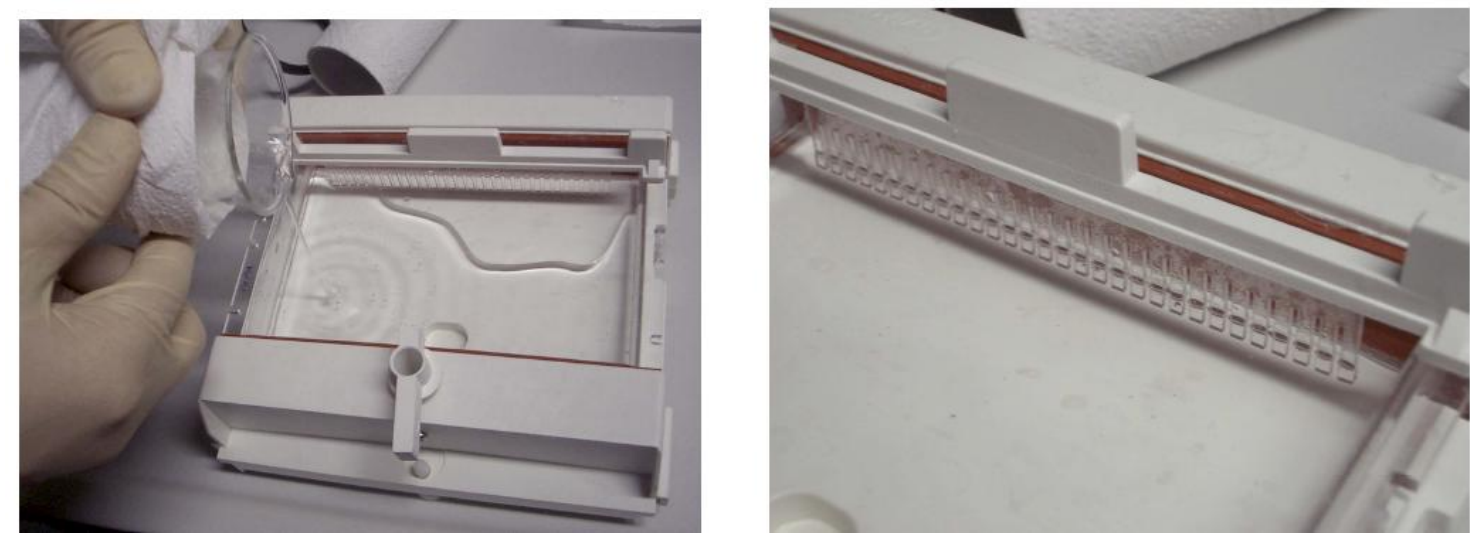

Figure VII.4.1. Boiling agarose solution is poured in the gel tray and a template comb is inserted. The gel then is allowed to cool down and solidify at RT.

Prior to loading into the wells of the gel, the NPs were first mixed with about $20 \%$ of volume with loading buffer ( $30 \%$ glycerol in $0.5 \times$ TBE with $0.3 \%$ Orange G). The 
glycerol increases the viscosity that the NP suspension can be better filled into the wells of the gel. The yellow/orange color of Orange $G$ allows for observing a fast migrating band as control on the gel. The NP suspension was carefully pipetted into the wells of the gel, i.e. into the holes made by the removed template comb, cf. Figure VII.4.2A. The wells should not be filled too high, and the NP suspension should not be too concentrated, as otherwise the band upon running electrophoresis may be smeared out. Once the gel had been loaded with NP suspension the electrophoresis devices was connected to a power source (BioRad Power Pac 1000) and run at a constant voltage of typically $100 \mathrm{~V}$, corresponding to a potential gradient of $10 \mathrm{~V} / \mathrm{cm}$. The NPs were then run on the $2 \%$ agarose gels for 30 - 100 min under the following conditions: $100 \mathrm{~V}, 500 \mathrm{~mA}, 250 \mathrm{~W}, 0.5 \times$ TBE buffer. In case the NPs are charged, they migrate towards the oppositely charged pole, $c f$. Figure VII.4.2B. The speed of migration depends on the charge and hydrodynamic diameter of the NPs. In this way different NP species can be separated as different bands on the gel. The more homogeneous the size- and charge-distribution of one NP species, she sharper the corresponding NP band is. The longer the running time, the better the separation of the NP bands.

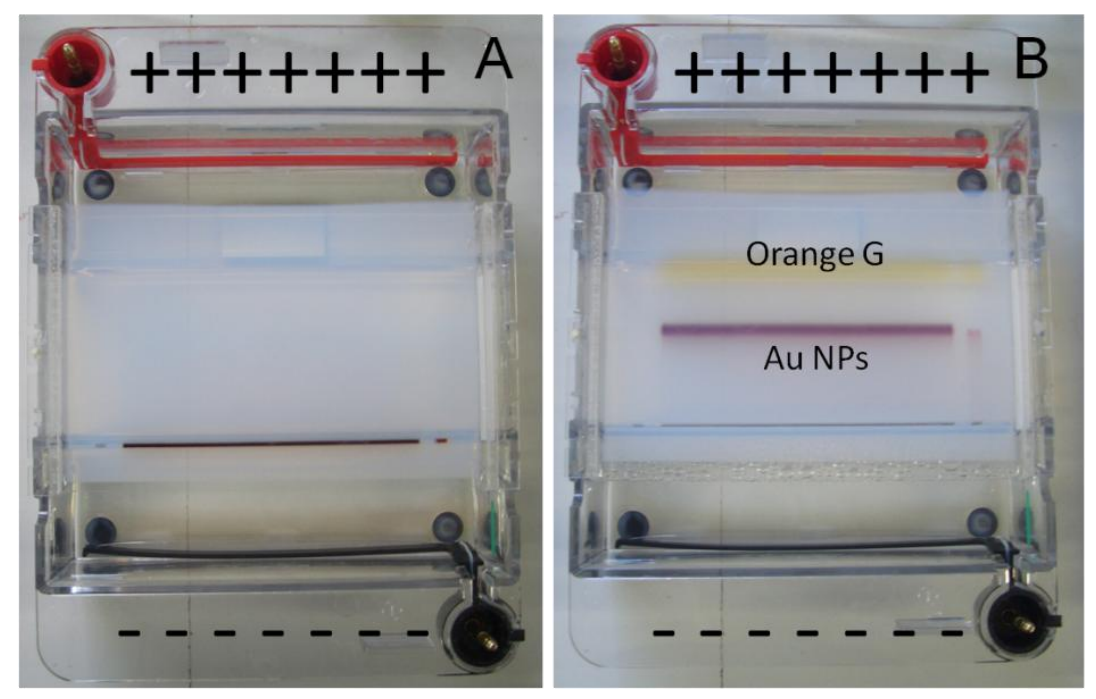

Figure VII.4.2. Photographs of an agarose gel filled with anionic Au NPs $(\approx 4 \mathrm{~nm}$ diameter, cf. (I.2, (VI.3) A) before and B) after electrophoresis. The NP sample is loaded in the well seen on the bottom of the image and can be seen by the red color of the Au NPs as labeled. PMA-coated Au NPs are negatively charged and thus they migrate towards the positive pole when an electric field is applied, i.e. in the shown image towards the top of the image. Orange $G$ is a small negatively charge molecule, and thus migrates faster than Au NPs towards the positive pole. Yellow and red bands in the gel correspond to the Orange $G$ and Au NPs, respectively. 
Afterwards, the gel was taken out from the tray and a digital picture was taken (BioRad Gel Doc), showing the gel under visible or UV light. In case the NPs did not migrate sufficiently, the gel was run for another $30 \mathrm{~min}$ and another photograph was taken after the additional running time. For imaging, the gel was placed on a white plastic plate to enhance the contrast and was inserted into the chamber of the imaging device, $c f$. Figure VII.4.3.

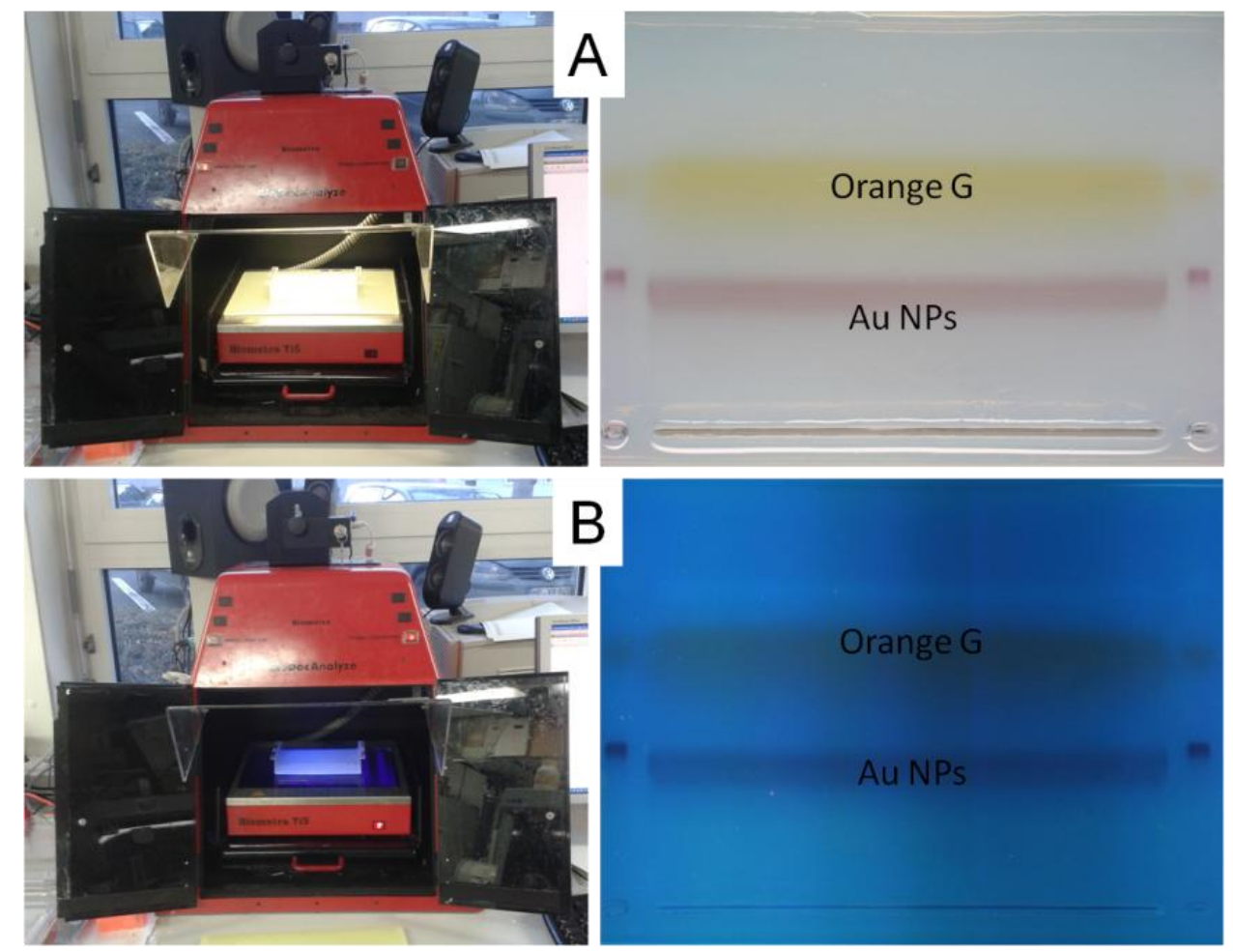

Figure VII.4.3. Photographs of the BioRad Gel Doc imaging device, which was used to take pictures of gel containing Orange $G$ and Au NPs bands (in Figure VIII.4.2), under white light $(A)$ or under UV illumination (B). In both $A$ and $B$ : The gel inside the imaging device is shown on the left and the resulting images on the right. In case of white light illumination the yellow band of Orange $G$ and the red band of Au NPs can be seen. Since Orange $G$ and the Au NPs are non-fluorescent, no fluorescence can be observed under UV illumination.

Gel electrophoresis can be used for purification of NP samples. Free impurities such as surfactants, molecules, or polymers micelles typically migrate faster than NPs. By taking out the NP band from the gel, an NP suspension purified from empty polymer micelles can be obtained. The band of the NPs was cut with a knife and then put into a dialysis bag tube (Spectra/Pro 6 dialysis tubing, $50 \mathrm{kDa}$ MWCO, $34 \mathrm{~mm}$ flat width, Spectralabs, \#132544). The gel-filled bag was then put into the electrophoresis cell filled with fresh $0.5 \times$ TBE buffer and voltage (100 V) was applied, typically for $20 \mathrm{~min}$. 
Due to the applied voltage the NPs migrated out of the gel, but they remained trapped inside the dialysis bags. Once the NPs had been migrated out of the gel pieces the voltage was switch off, the dialysis bags were taken out of the electrophoresis cell, and the suspension of NPs was pipetted out of the bags, $c f$. Figure VII.4.4. For concentration and buffer exchange of this NP suspension ultrafiltration was applied as reported in §VII.2.

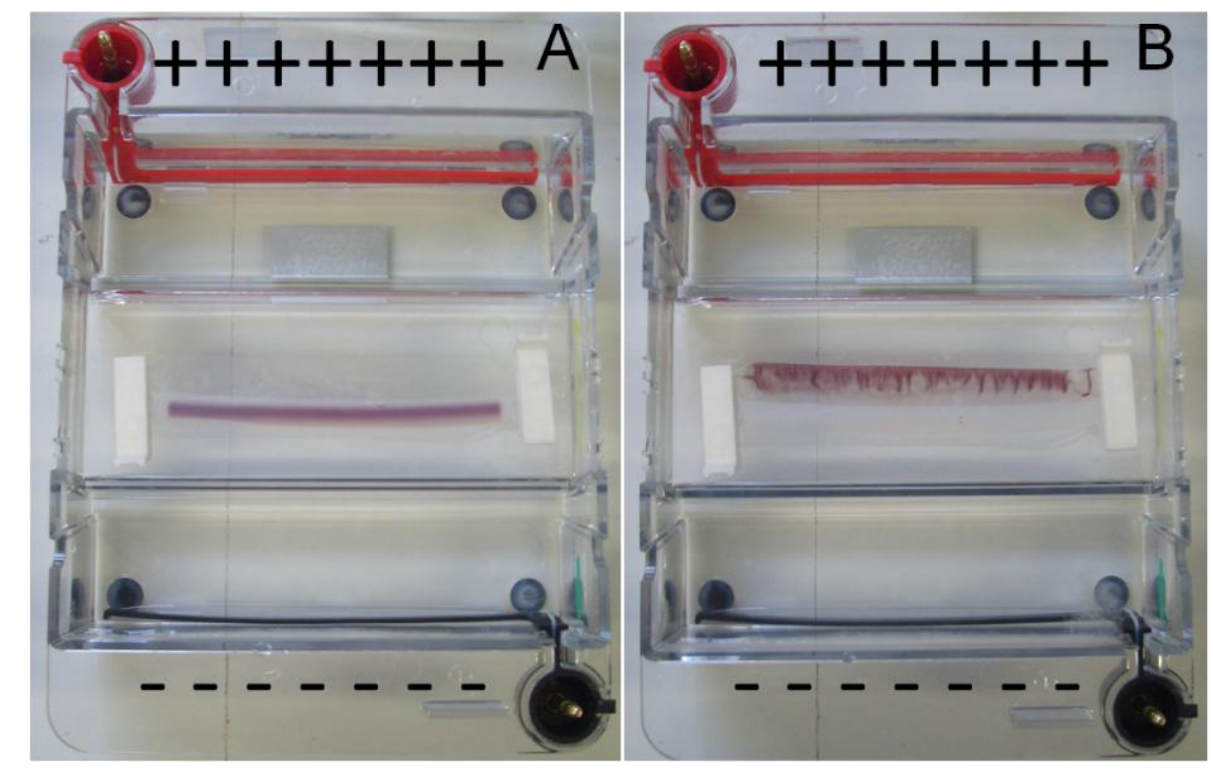

Figure VII.4.4. Recovering Au NPs from a gel band. A) The extracted band contains Au NPs ( $\approx 4 \mathrm{~nm}$ diameter, cf. §I.2, §VI.3) and was placed inside a dialysis bag, which was placed into the electrophoresis cell. B) After applying an electric field the Au NPS migrated out of the gel band, but remained trapped inside the dialysis bag.

\section{VII.5 Ultracentrifugation}

A major method for NP purification is centrifugation. NPs with density higher than the density of the solvent may be precipitated by gravity or centrifugal forces, whereas lighter impurities (surfactants, ions, capping agents, polymers, etc.) remain in the supernatant and thus can be removed by supernatant decantation. The NP pellet is then resuspended in fresh medium/solvent, $c f$. Figure VII.5.1. This procedure works well for NPs that are large or dense enough to be precipitated by using a tabletop centrifuge (e.g. Au NPs of $\mathrm{d}_{\mathrm{c}}>15 \mathrm{~nm}$ at $10000 \mathrm{rcf}$ ). In case of smaller NPs ultracentrifugation with much higher centrifugation speed is needed. The duration and centrifugal forces depend on the NPs' size, shape, and density, as well as on solventrelated parameters such as solvent density/viscosity. Moreover, the colloidal stability of NPs during centrifugation is a very important parameter to be considered when 
selecting the centrifugation settings. Collectively, optimum centrifugation settings for a specific NP suspension should be evaluated experimentally. For example, PMA-gdodecyl capped Au NPs ( $\approx 4 \mathrm{~nm}$ core diameter, cf. §I.2, §VI.3) required centrifugation at $85000 \mathrm{rcf}$ (Thermo Scientifiv ultracentrifuge equiped with a SureSpin 630 rotor in combination with $36 \mathrm{~mL}$ PET thin-walled tubes (Thermo Scientific, \#75000471)) for $3 \mathrm{~h}$. After centrifugation, a red pellet was observed and the supernatant was discarded. Three centrifugation cycles were found to be necessary to purify PMA-g-dodecyl capped Au NPs from empty PMA-g-dodecyl micelles (cf. Figure VII.5.1). After the third centrifugation step, the Au NPs were suspendet in milliQ water and filtered through a hydrophilic syringe filter $(0.22 \mu \mathrm{m}, c f$. $\S \mathrm{VII} .2)$.

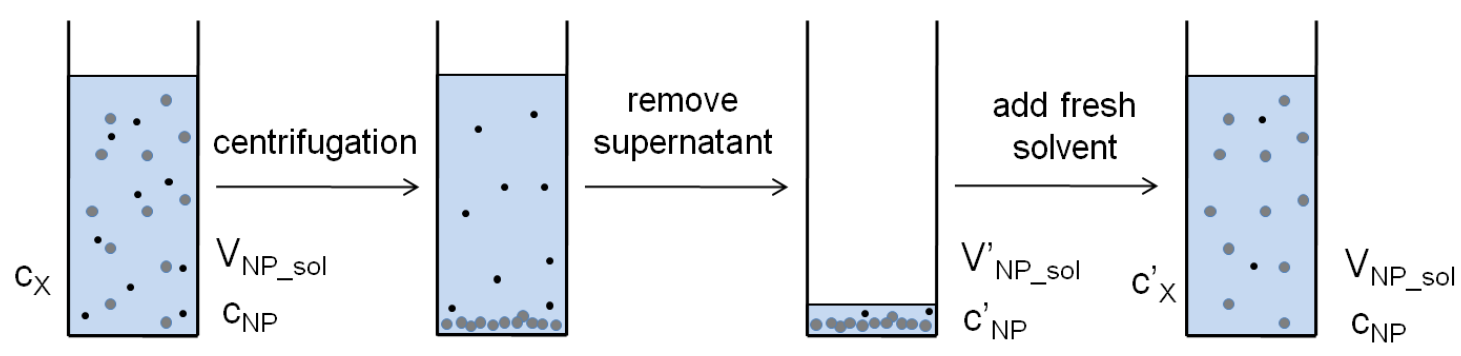

Figure VII.5.1. Purification via repeated centrifugation of a suspension of NPs (drawn in gray) of initial volume $V_{N P_{-} \text {sol }}$ and a concentration of $c_{N P}$. Small impurities (drawn in black) with concentration $c_{X}$ are presented initially in the suspension. Upon centrifugation the NPs form a pellet, whereas the impurities remain in solution. The supernatant with the impurities is removed. Fresh solvent is added to result in a decreased final concentration of impurities $c^{\prime}{ }_{X}=c_{X} \cdot V^{\prime}{ }_{N P_{-} s o l} / V_{N P_{-} s o l}$.

Various purification methods may result in different purification efficiency. For example, we did find that purification of PMA-g-dodecyl capped Au NPs using ultracentrifugation is more efficient than gel electrophoresis method. Interestingly, some empty polymer micelles may remain in solution after electrophoresis, whereas the NP sample was virtually free of empty micelles after three ultracentrifugation cycles, cf. Figure VII.5.2. 
A

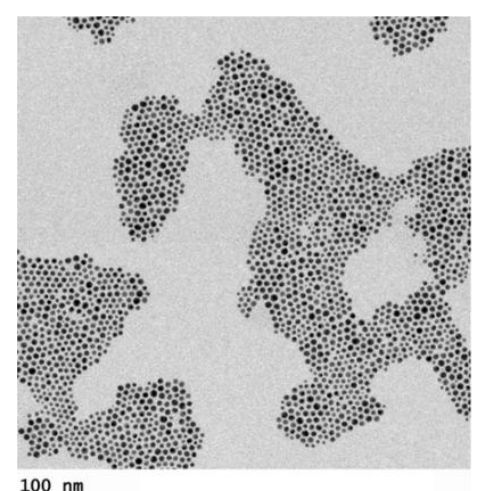

$\underline{100 \mathrm{~nm}}$

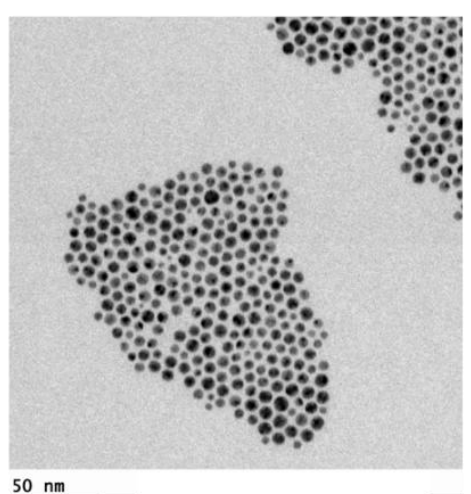

B

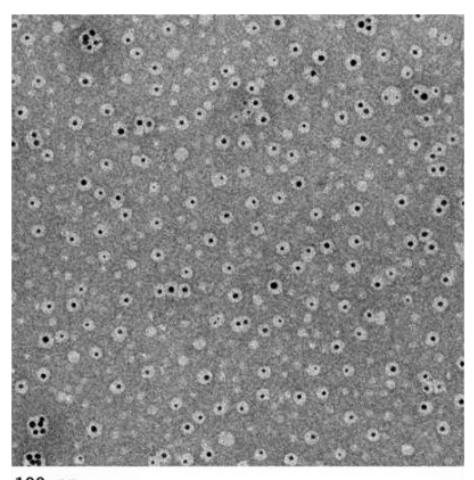

$100 \mathrm{~nm}$

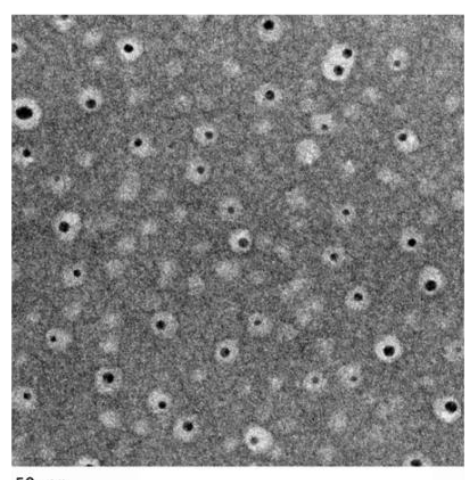

$50 \mathrm{~nm}$

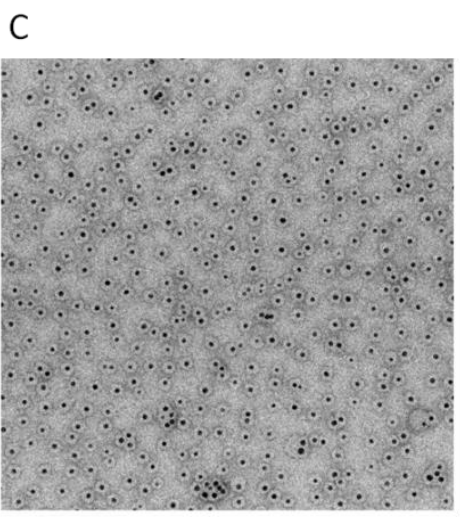

$100 \mathrm{~nm}$

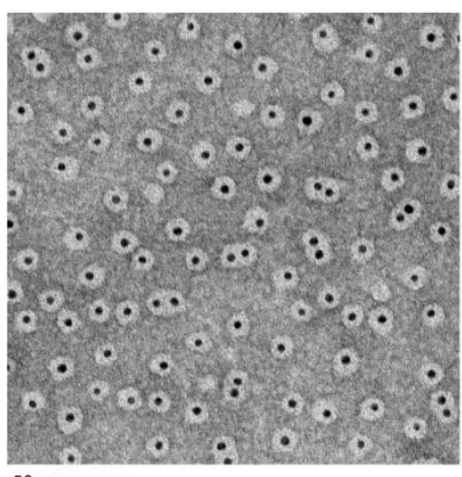

$\underline{50 \mathrm{~nm}}$

Figure VII.5.2. Transmission electron microscopy (TEM) images of PMA-g-dodecyl capped Au NPs ( $\approx 4 \mathrm{~nm}$ core diameter, cf. §I.2. A) TEM images of the DDT-Au NPs (cf. \&VIII.3) before coating with PMA-g-dodecyl polymer at two magnifications in upper and lower panels. B) TEM images after negative staining (cf. \&VIII.4) of the PMA-g-dodecyl capped Au NPs purified by gel electrophoresis (cf. \&VII.4) at two magnifications in upper and lower panels (Note: some empty polymer micelles can be seen (gray spots without black NP inside)). C) TEM images of the PMA-g-dodecyl capped Au NPs purified by ultracentrifugation at $65000 \times g$ three times at two magnifications in upper and lower panels (Note: the sample is virtually free of empty polymer micelles). The scale bars in the top and bottom row represent $100 \mathrm{~nm}$ and $50 \mathrm{~nm}$, respectively. 


\section{Characterization of NPs' structural properties}

\section{VIII.1 General comments about the composition of the NPs}

VIII.2 Determination of NP concentrations by elemental analysis

VIII.3 Transmission electron microscopy of NP cores

VIII.4 Transmission electron microscopy of NPs including their organic shell

VIII.5 X-ray diffraction

VIII.6 Thermogravimetric analysis

VIII.7 Infrared spectroscopy

VIII.8 Nuclear Magnetic Resonance

\section{VIII.1 General comments about the composition of the NPs}

As outlined in sections I - VI, NPs described in this report are composed of an inorganic core and an organic shell. The total mass of one NP $\left(m_{N P}\right)$ involves the mass of the inorganic core $\left(m_{c}\right)$ and the mass of the organic surface coating. As inorganic cores can be conveniently imaged with transmission electron microscopy (TEM, $c f$. §VIII.3), the mass of one NP core can be obtained. In addition to the NPs' core material density, the NP core volume $V_{c}$ should be calculated using NP dimensions as obtained from TEM images (core diameter $\left(d_{c}\right)$ in case of spherical NPs and core diameter $\left(d_{c}\right)$ and length $\left(L_{c}\right)$ in the case of rod-shaped NPs). Note, that without additional staining (cf. §VIII.4) the organic shell does not provide contrast in regular TEM, and thus only the inorganic cores are visible. In case of spheres and rods (assumed to have the shape of a cylinder) the volume of one NP core is:

$$
\begin{array}{r}
\mathrm{V}_{\mathrm{c}}(\text { sphere })=\left(\frac{4 \pi}{3}\right) \times\left(\frac{\mathrm{d}_{\mathrm{c}}}{2}\right)^{3}=\left(\frac{\pi}{6}\right) \times \mathrm{d}_{\mathrm{c}}{ }^{3} \quad \text { (Equation VIII.1.1) } \\
\mathrm{V}_{\mathrm{c}}(\operatorname{rod})=\pi \times\left(\frac{\mathrm{d}_{\mathrm{c}}}{2}\right)^{2} \times \mathrm{L}_{\mathrm{c}}=\left(\frac{\pi}{4}\right) \times \mathrm{d}_{\mathrm{c}}{ }^{2} \times \mathrm{L}_{\mathrm{c}} \quad \text { (Equation VIII.1.2) }
\end{array}
$$

If the core is built in core/shell geometry (cf. Figure I.1.1), the volume $\mathrm{V}_{c 2}$ of the shell is

$$
\mathrm{V}_{\mathrm{c} 2}(\text { sphere })=\mathrm{V}_{\mathrm{c} 12}(\text { sphere })-\mathrm{V}_{\mathrm{c} 1}(\text { sphere })=\left(\frac{\pi}{6}\right) \times\left(\mathrm{d}_{\mathrm{c} 12}{ }^{3}-\mathrm{d}_{\mathrm{c} 1}{ }^{3}\right)
$$

(EquationVIII.1.3)

In case the core is composed out of material of density $\rho_{c}$, the mass of one NP core is:

$$
\mathrm{m}_{\mathrm{c}}=\rho_{\mathrm{c}} \times \mathrm{V}_{\mathrm{c}}
$$


For most inorganic NP materials their densities can be found in the literature. In first order the bulk densities of the materials can be assumed. The molar mass of an NP core thus is:

$$
\mathrm{M}_{\mathrm{c}}=\mathrm{m}_{\mathrm{c}} \times \mathrm{N}_{\mathrm{A}}
$$

In case the core is composed out of one element $X$, then each NP core includes $N_{X / N P}$ atoms of $X . M_{X}$ is the molar mass of element $X$.

$$
\mathrm{N}_{\mathrm{X} / \mathrm{NP}}=\frac{\mathrm{M}_{\mathrm{c}}}{\mathrm{M}_{\mathrm{X}}}=\rho_{\mathrm{c}} \times \mathrm{V}_{\mathrm{c}} \times \frac{\mathrm{N}_{\mathrm{A}}}{\mathrm{M}_{\mathrm{X}}}
$$

To give an example, for a spherical Au NP with core diameter $d_{c}=4.2 \mathrm{~nm}, V_{c}=(\pi / 6) \times$ $(4.2 \mathrm{~nm})^{3} \approx 38.8 \mathrm{~nm}^{3}$. Given the bulk density of Au of $\rho_{A u}=19.3 \mathrm{~g} / \mathrm{cm}^{3}$, the mass of one Au core thus is $m_{c}=19.3 \mathrm{~g} / \mathrm{cm}^{3} \times 38.8 \mathrm{~nm}^{3} \approx 750 \mathrm{~g} \cdot\left(10^{-9} \mathrm{~m} / 10^{-2} \mathrm{~m}\right)^{3}=7.5 \times 10^{-19} \mathrm{~g}$. The molar mass of a Au core thus is $M_{c}=m_{c} \times N_{A}=7.5 \cdot 10^{-19} \mathrm{~g} \cdot 6.02 \times 10^{23} \mathrm{~mol}^{-1} \approx 4.5 \times 10^{5}$ $\mathrm{g} / \mathrm{mol}$. Given the molar mass of $\mathrm{Au} \mathrm{M}_{\mathrm{Au}}=197 \mathrm{~g} / \mathrm{mol}$, one $\mathrm{Au}$ core thus comprises approximately $\mathrm{N}_{\mathrm{Au} / \mathrm{NP}}=\mathrm{M}_{\mathrm{c}} / \mathrm{M}_{\mathrm{Au}}=4.5 \times 10^{5} \mathrm{~g} \cdot \mathrm{mol}^{-1} / 197 \mathrm{~g} \cdot \mathrm{mol}^{-1} \approx 2284 \approx 2300$ Au atoms.

In case the core is composed out of two elements $X$ and $Y$ with the stoichiometry $X_{x} Y_{y}$ (i.e. for $\mathrm{Fe}_{3} \mathrm{O}_{4} \mathrm{X}=3$ and $\mathrm{y}=4$ ), then each NP core includes $\mathrm{N}_{\mathrm{X} / \mathrm{NP}}$ and $\mathrm{N}_{\mathrm{Y} / \mathrm{NP}}$ atoms of $\mathrm{X}$ and $Y$, respectively. $M_{X}$ and $M_{Y}$ are the molar masses of the two elements.

$$
\begin{gathered}
M_{c}=N_{X / N P} \times M_{X}+N_{Y / N P} \times M_{Y} \\
\frac{N_{X / N P}}{N_{Y / N P}}=\frac{x}{y}
\end{gathered}
$$

Thus $M_{c}=N_{x / N P} \times M_{X}+N_{x / N P} \times(y / x) \times M_{y}$, leading to

$$
\begin{gathered}
N_{X / N P}=\frac{M_{c}}{\left(M_{X}+\left(\frac{y}{x}\right) \times M_{Y}\right)}=\rho_{c} \times V_{c} \times \frac{N_{A}}{\left(M_{X}+\left(\frac{y}{x}\right) \times M_{Y}\right)} \\
N_{Y / N P}=\frac{M_{c}}{\left(M_{Y}+\left(\frac{x}{y}\right) \times M_{x}\right)}=\rho_{c} \times V_{c} \times \frac{N_{A}}{\left(M_{y}+\left(\frac{x}{y}\right) \times M_{x}\right)}
\end{gathered}
$$

To give an example, for a spherical $\mathrm{Fe}_{3} \mathrm{O}_{4} \mathrm{NP}$ with core diameter $\mathrm{d}_{\mathrm{c}}=4.2 \mathrm{~nm}, \mathrm{~V}_{\mathrm{c}}=$ $(\pi / 6) \times(4.2 \mathrm{~nm})^{3} \approx 38.8 \mathrm{~nm}^{3}$. Given the bulk density of $\mathrm{Fe}_{3} \mathrm{O}_{4}$ of $\rho_{\mathrm{Fe} 304}=5 \mathrm{~g} / \mathrm{cm}^{3}$, the mass of one $\mathrm{Fe}_{3} \mathrm{O}_{4}$ core thus is $\mathrm{m}_{\mathrm{c}}=5 \mathrm{~g} / \mathrm{cm}^{3} \times 38.8 \mathrm{~nm}^{3}=194 \mathrm{~g} \times\left(10^{-9} \mathrm{~m} / 10^{-2} \mathrm{~m}\right)^{3} \approx$ $1.9 \times 10^{-19} \mathrm{~g}$. The molar mass of a $\mathrm{Fe}_{3} \mathrm{O}_{4}$ core thus is $\mathrm{M}_{\mathrm{c}}=\mathrm{m}_{\mathrm{c}} \times \mathrm{N}_{\mathrm{A}}=1.9 \times 10^{-19} \mathrm{~g} \cdot 6.02 \cdot 10^{23}$ 
$\mathrm{mol}^{-1} \approx 1.2 \times 10^{5} \mathrm{~g} / \mathrm{mol}$. Given the molar masses of iron $\mathrm{M}_{\mathrm{Fe}}=55.8 \mathrm{~g} / \mathrm{mol}$ and of oxygen $\mathrm{M}_{\mathrm{O}}=16 \mathrm{~g} / \mathrm{mol}$, one $\mathrm{Fe}_{3} \mathrm{O}_{4}$ core thus comprises approximately of $\mathrm{N}_{\mathrm{Fe} / \mathrm{NP}}=\mathrm{M}_{\mathrm{c}} /\left(\mathrm{M}_{\mathrm{Fe}}+\right.$ $\left.(4 / 3) \times \mathrm{M}_{\mathrm{O}}\right)=1.2 \times 10^{5} \mathrm{~g} \cdot \mathrm{mol}^{-1} /\left(55.8 \mathrm{~g} \cdot \mathrm{mol}^{-1}+(4 / 3) \times 16 \mathrm{~g} \cdot \mathrm{mol}^{-1}\right)=1556 \approx 1600$ iron atoms, and $\mathrm{N}_{\mathrm{O} / \mathrm{NP}}=\mathrm{M}_{\mathrm{C}} /\left(\mathrm{M}_{\mathrm{O}}+(3 / 4) \times \mathrm{M}_{\mathrm{Fe}}\right)=1.2 \times 10^{5} \mathrm{~g} \cdot \mathrm{mol}^{-1} /\left(16 \mathrm{~g} \cdot \mathrm{mol}^{-1}+(3 / 4) \times 55.8 \mathrm{~g} \cdot \mathrm{mol}^{-1}\right)=$ $2074 \approx 2100$ oxygen atoms.

\section{VIII.2 Determination of NP concentrations by elemental analysis}

With inductively coupled plasma coupled with either mass spectrometry (ICP-MS) or optical emission spectroscopy (ICP-OES), quantitative elemental analysis can be performed. Dilution and acid digestion are required prior to analysis. For example, in the case of metal NPs, such as Au or Ag, $50 \mu \mathrm{L}$ of the sample was added to $200 \mu \mathrm{L}$ of aqua regia (i.e. concentrated $\mathrm{HCl}(35 \mathrm{wt} \%)$ and $\mathrm{HNO}_{3}$ (67 wt\%) in 3:1 volume ratio) and then it was left for digestion (oxidation of metallic cores into their corresponding ions) for about $30 \mathrm{~min}$. The digestion step diluted the sample by a dilution factor of $5 \mathrm{x}$ prior to further dilution by a factor of $10 \mathrm{x}$ with $\mathrm{HCl}$ solution $(2 \mathrm{wt} \% \mathrm{HCl}$ ) prior to their injection into ICP-MS (total dilution factor of 50x). A high dilution factor in acidic media is required to stabilize metallic ions and to improve the analytical detection limit, as well as to ensure a qualitative and quantitatively equal background signal for all samples. Samples with high levels of acid might harm the ICP-MS machinery.

For calibration, the auto-tuning solution from Agilent for ICP-MS 7500cs with a standard concentration of $1 \mathrm{\mu g} / \mathrm{L}$ of $\mathrm{Ce}, \mathrm{Co}, \mathrm{Li}, \mathrm{Mg}, \mathrm{Tl}$, and $\mathrm{Y}$ was used to set the general background, as well as to calibrate the electrical field of the lenses and the magnetic quadrupole field in strength and frequency before the actual run. The oxidation level (less than $0.8 \%$ ), double charge rate (less than $1.8 \%$ ) was also measured using this tuning solution.

All vials and working materials were either cleaned using freshly prepared aqua regia for $2 \mathrm{~h}$ followed by boiling in Milli-Q water, or consisted of sterile and clean nonreusable consumables. The samples were introduced into the ICP-MS set-up through a Perfluoroalkoxy-Alkane (PFA) based microflow spray chamber, where the aqueous sample was nebulized, introduced to the argon gas flow, and transported to the torch, where it was ionized in an argon-plasma of around $6000^{\circ} \mathrm{C}$. After ionization the sample was presorted using omega lens, element-wise separated in the quadrupole field through the mass to charge ratio, again sorted using kinetic barriers and a charged lens system, and finally detected with either an analog or a digital detector depending on the count rate. It is important to mention that the working mode of the instrument and flow of Ar gas also affected the analysis. 
Before each run, a proper calibration was needed with an appropriate standard. The ICP-MS set-up was calibrated by using an Agilent standard of corresponding ions (all the standards of corresponding ions should be available prior to the measurement). For example, in the case of Au NPs the element to be detected is Au. The Agilent Au standard has $1000 \mathrm{mg} / \mathrm{L}$ Au concentration from which eight solutions of concentration $2500,1000,500,250,100,50,25$, and 10 parts per billion $\left(1 \mathrm{ppb}=10^{-9}=1 \mu \mathrm{g}\right.$ detected $\mathrm{Au} / 1 \mathrm{~kg}$ water (density $\rho_{\mathrm{H} 2 \mathrm{O}}=1 \mathrm{~kg} / \mathrm{L}$ ), corresponding to a gold concentration $\mathrm{C}_{\mathrm{Au}}=1$ $\mu \mathrm{g} / \mathrm{L}$ ) were prepared along with a blank using the same low matrix (aqueous $2 \mathrm{wt} \%$ $\mathrm{HCl}$ ) as diluent. The calibration curve was constructed using concentration points of the aforementioned standard solutions. Syringe and tubings were washed thoroughly at the end of each run (for gold, extensive rinsing is needed as it often sticks to the tube). The calibration curve and the sample results were used to calculate the concentration of measured elements in the corresponding samples considering the used dilution factor (Final concentration = dilution factor $\mathrm{x}$ measured concentration).

With TEM studies the volume of one NP core $V_{c}$ (the core that contains the detected element) can be determined, leading to the total number of atoms $X$ per NP ( $\mathrm{N}_{\mathrm{X} / \mathrm{NP}}$ ) ( $c f$. $\S$ VIII.3). By knowing the elemental concentration of metallic ions $X$ in a sample $c_{x}$, the concentration of NPs in the sample can be determined:

$$
\mathrm{C}_{\mathrm{NP}}=\mathrm{C}_{\mathrm{x}} / \mathrm{N}_{\mathrm{X} / \mathrm{NP}}
$$

(Equation VIII.2.1)

For example, to determine the concentration of $\mathrm{Fe}_{3} \mathrm{O}_{4} \mathrm{NP}$ in a sample ( $\mathrm{C}_{\mathrm{NP}}$ ) we need to determine the elemental concentration of iron $\mathrm{C}_{\mathrm{Fe}}$ in the sample and the number of $\mathrm{Fe}$ atoms per NP as per Equation VIII.1.9: $N_{\mathrm{Fe} / \mathrm{NP}}=\rho_{\mathrm{Fe} 304} \times \mathrm{V}_{\mathrm{c}} \times \mathrm{N}_{\mathrm{A}} /\left(\mathrm{M}_{\mathrm{Fe}}+(4 / 3) \times \mathrm{M}_{\mathrm{O}}\right)=$ $\rho_{\mathrm{Fe} 304} \times(\pi / 6) \times d_{c}{ }^{3} \times V_{c} \times N_{A} /\left(M_{\mathrm{Fe}}+(4 / 3) \cdot \mathrm{M}_{\mathrm{O}}\right)$. The concentration of $\mathrm{Fe}_{3} \mathrm{O}_{4} \mathrm{NP}$ in the sample now can be calculated as follows: $\mathrm{c}_{\mathrm{NP}}=\mathrm{c}_{\mathrm{Fe}} / \mathrm{N}_{\mathrm{Fe} / \mathrm{NP}}$.

\section{VIII.3 Transmission electron microscopy of NP cores}

Transmission Electron Microscopy (TEM) analysis allows a direct measure of the size and shape of NPs. The organic ligand shell typically does not provide sufficient electron contrast, and thus in TEM images only the inorganic part of the NPs can be seen. However, special techniques can be employed to visualize the organic shell around NPs as are described in §VIII.4.

All images in this section were obtained using a Philips CM 100 electron microscope operated at an accelerating voltage of $100 \mathrm{kV}$ or on a JEOL JEM-1400PLUS TEM 
operated at an accelerating voltage of $120 \mathrm{kV}$. The NPs were deposited from a dilute solution onto a 3-4 nm thick film of amorphous carbon supported on a 400 mesh copper grid (Ted Pella Inc., \#01822-F). One drop ( $2 \mu \mathrm{L}$ ) of NP suspension was deposited onto the grid and the solvent was evaporated at RT. In the case of NPs suspended in water the grids were kept under ethanol vapor for 1-2 days prior to sample deposition. This treatment made the carbon film more hydrophilic and improved the adsorption of the NPs on it. The microscope magnification was calibrated with a calibration grid (Grating Replica, Waffle, 2160 I/mm, on 3mm grid, Ted Pella Inc., \#607).

From TEM images several parameters can be extracted. First, the shape of the NPs can be determined, which is important in the case of non-spherical NPs such as rods, stars, etc. Second, the frequency distribution (histograms) of NP core diameter $d_{c}$ (and length $L_{c}$ in case of rod-shaped NPs) can be determined to calculate the NP's dimensions. Figure VIII.3.1-13 show TEM images of NP samples prepared in this work, along with their size distribution histograms. To determine the size distribution histograms, dimensions of several NPs were measured from their TEM images using ImageJ 1.42 (freely available software from https://imagej.nih.gov/ij/). As a representative sample, at least 100 NPs were analyzed for each size distribution histogram, from which the mean diameter of NPs $\left(d_{c}\right)$ and its standard deviation (as indicator of polydispersity) could be calculated. Third, the state of agglomeration can be observed (note that TEM works with dried samples, and thus even NPs, which are individually dispersed in solution, may cluster on the TEM grids due to drying-induced assembly). Dryinginduced clustering on the TEM grids can be reduced by depositing diluted NP suspensions on the TEM grids, using treated grids (cationic or anionic surfaces), or optimizing the rate of drying. In contrast, when TEM grids are prepared with higher concentration of NPs, the NPs can self-assemble into 2-dimensional lattices, $c f$. Figure VIII.3.1. Forth, from TEM images with self-assembled NPs the thickness of the organic coating/shell around the inorganic NP cores can be estimated as half the minimum distance between the two adjacent inorganic NP cores ${ }^{71,74,92}$. However, for TEM imaging the NPs have to be deposited on a substrate and the solution in which the NPs are dispersed must be evaporated. The thickness of the organic layer is likely to be reduced due to the drying-induced shrinkage. For example, organic hydrophobic tails of two adjacent NPs can intercalate and polymeric shells on NPs can shrink upon desolvation. For these reasons, the measured thickness of the organic coating around NP cores as determined from TEM is likely to be underestimated, and therefore, the values obtained in this way have to be interpreted as lower limits. 
A

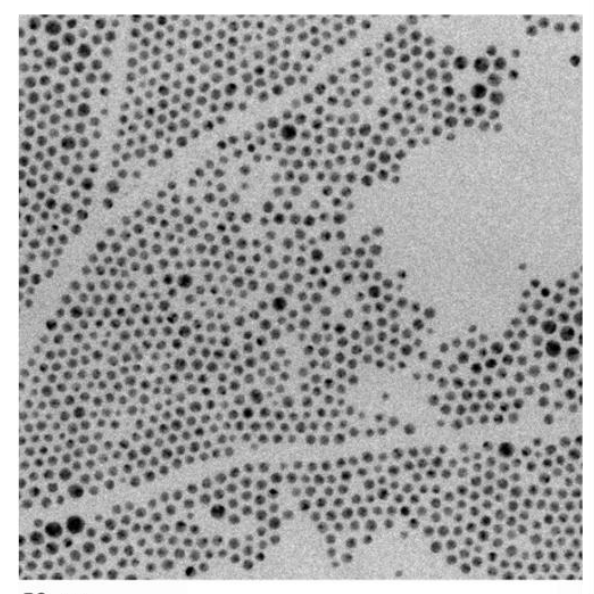

$50 \mathrm{~nm}$
B

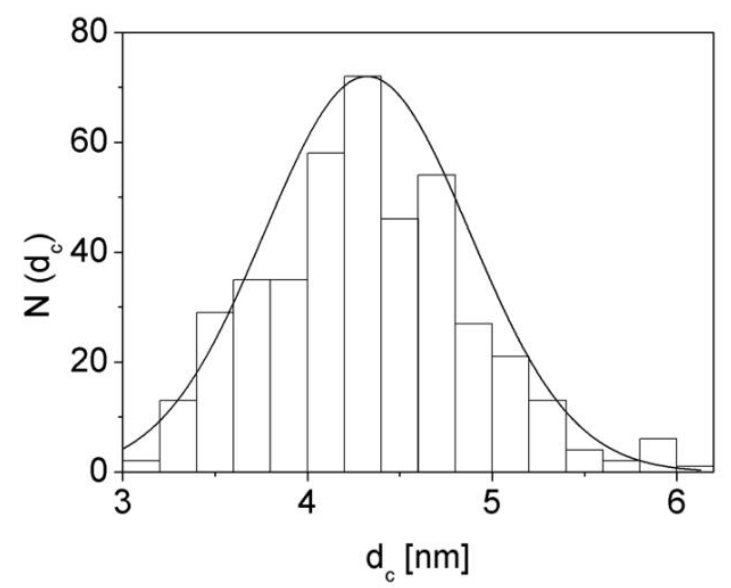

Figure VIII.3.1. A) TEM image of hydrophobic DDT-Au NPs (capped with dodecanethiol, $\approx 4 \mathrm{~nm}$ core diameter, $c f$. §I.2) dried on grid from a chloroform suspension. The scale bar corresponds to $50 \mathrm{~nm}$. B) Size distribution histogram, plotted as number of NPs $N\left(d_{c}\right)$ that have a core diameter of $d_{c}$. From this histogram the mean NP diameter and its standard deviation were determined to be $d_{c}=(4.3 \pm 0.4) \mathrm{nm}$.
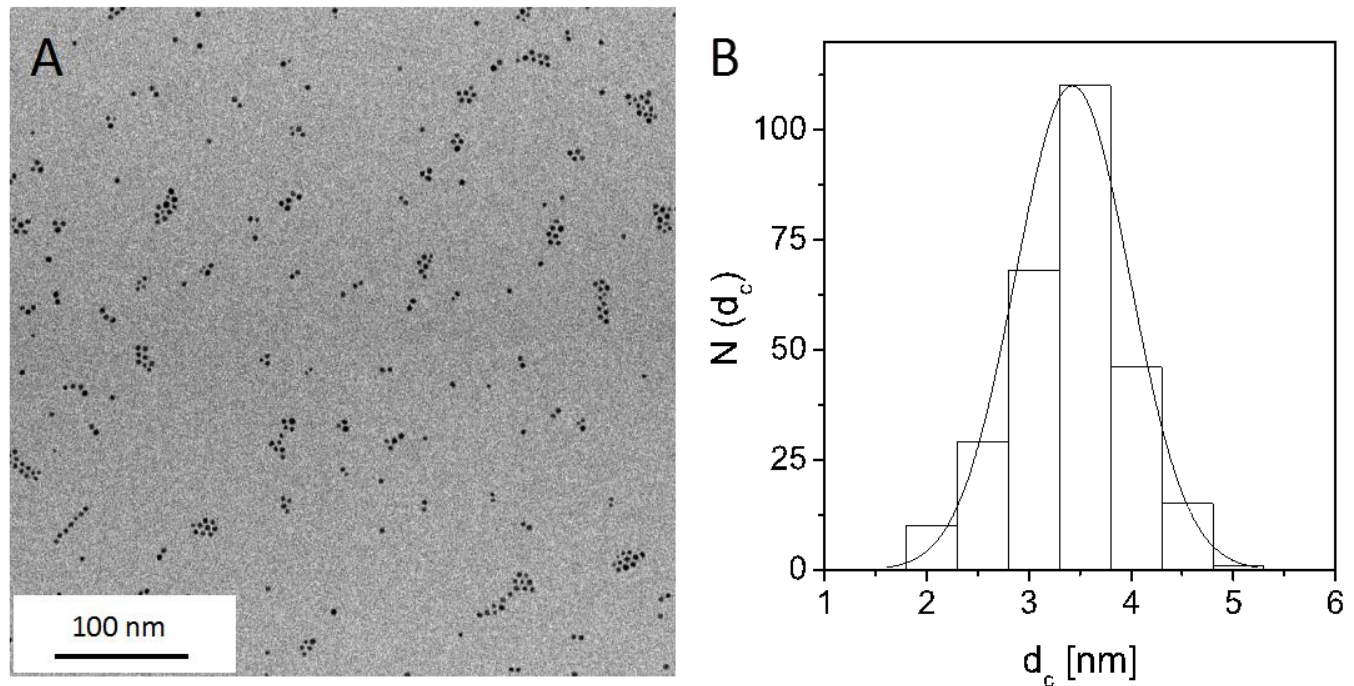

Figure VIII.3.2. A) TEM image of hydrophobic FePt NPs (capped with OA and OLA, $\approx 4$ $\mathrm{nm}$ core diameter, cf. \$l.4) dried on grid from a chloroform suspension. The scale bar corresponds to $100 \mathrm{~nm}$. B) Size distribution histogram, plotted as number of $N P s N\left(d_{c}\right)$ that have a core diameter of $d_{c}$. From this histogram the mean NP diameter and its standard deviation were determined to be $d_{c}=(3.4 \pm 0.6) \mathrm{nm}$. 

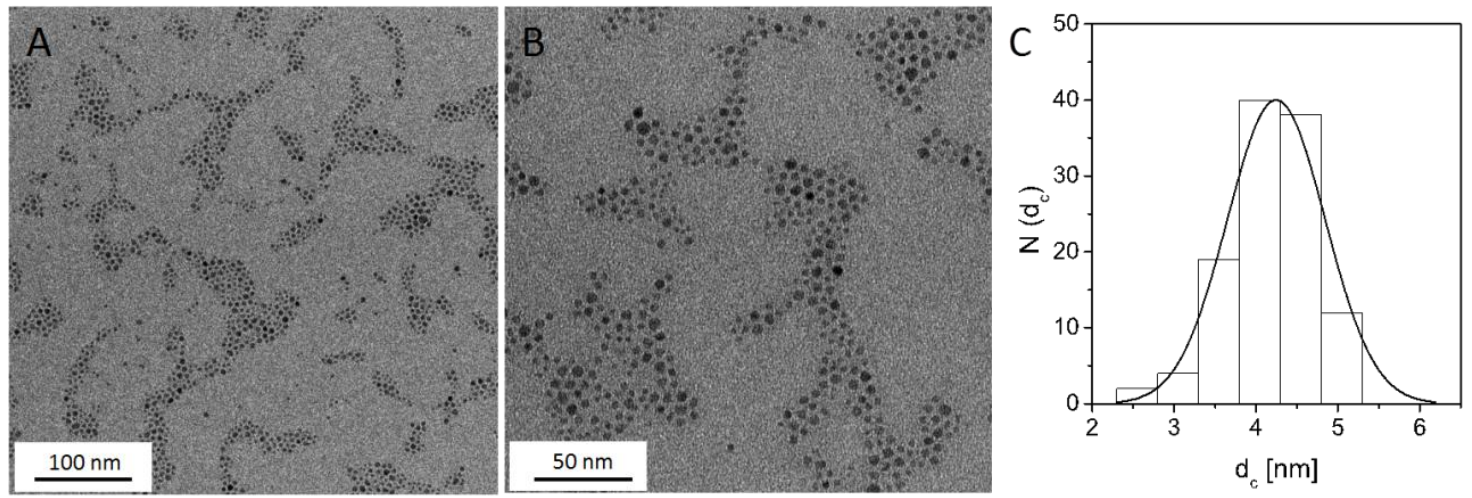

Figure VIII.3.3. TEM image of hydrophobic $\mathrm{Fe}_{3} \mathrm{O}_{4} \mathrm{NPs}$ (capped with OA and OLA, $\approx 4 \mathrm{~nm}$ core diameter, cf. §l.5) dried on grid from a chloroform suspension. A) The scale bar corresponds to $100 \mathrm{~nm}$ and $B$ ) the scale bar corresponds to $50 \mathrm{~nm}$. C) Size distribution histogram, plotted as number of NPs $N\left(d_{c}\right)$ that have a core diameter of $d_{c}$. From this histogram the mean NP diameter and its standard deviation were determined to be $d_{c}$ $=(4.2 \pm 0.6) \mathrm{nm}$.

A

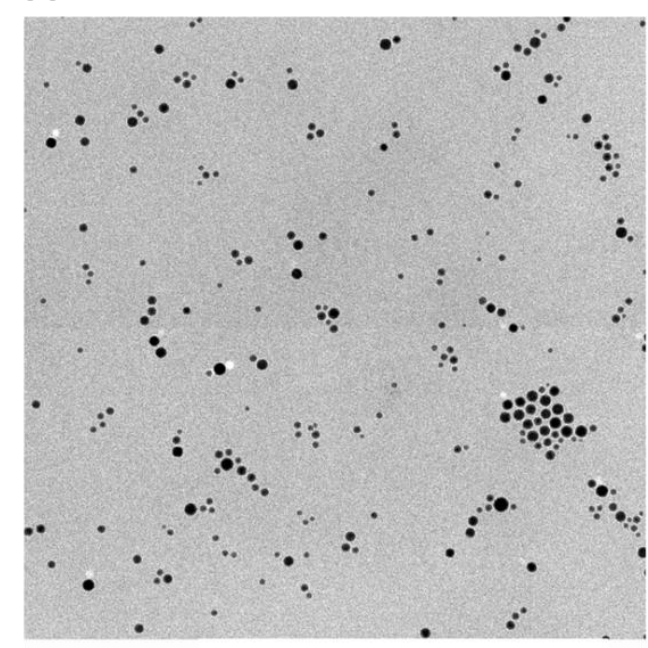

$200 \mathrm{~nm}$

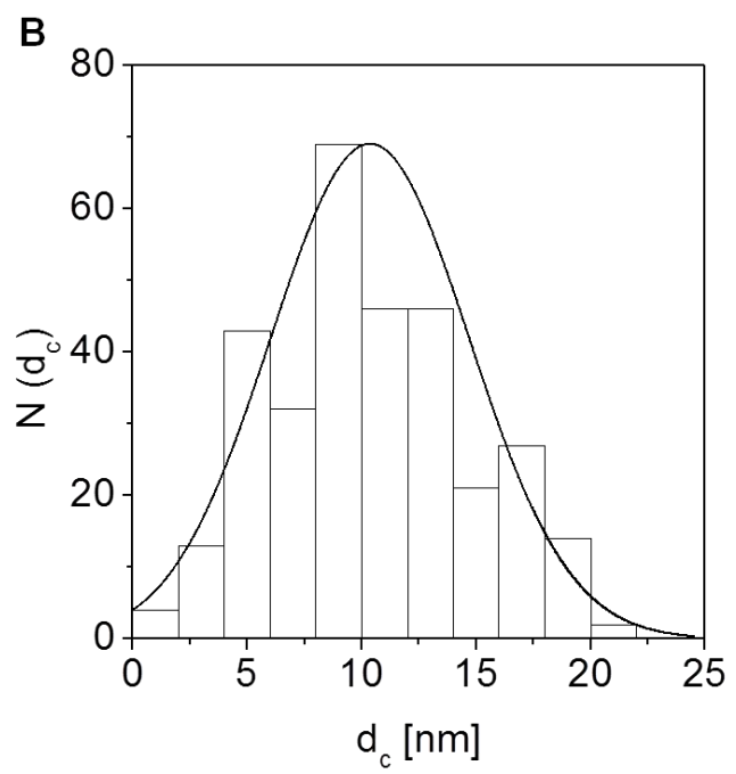

Figure VIII.3.4 A) TEM image of hydrophobic Sn NPs (capped with oleic acid, $\approx 11 \mathrm{~nm}$ core diameter, cf. \$l.6) dried on grid from a chloroform suspension. The scale bar corresponds to $200 \mathrm{~nm}$. B) Size distribution histogram, plotted as number of $N P s N\left(d_{c}\right)$ that have a core diameter of $d_{c}$. From this histogram the mean NP diameter and its standard deviation were determined to be $d_{c}=(10 \pm 4) \mathrm{nm}$. 
A1

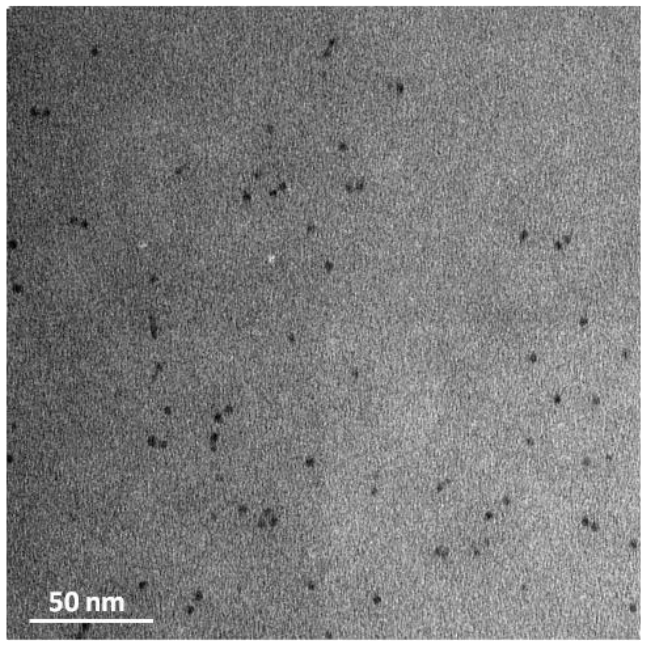

A2

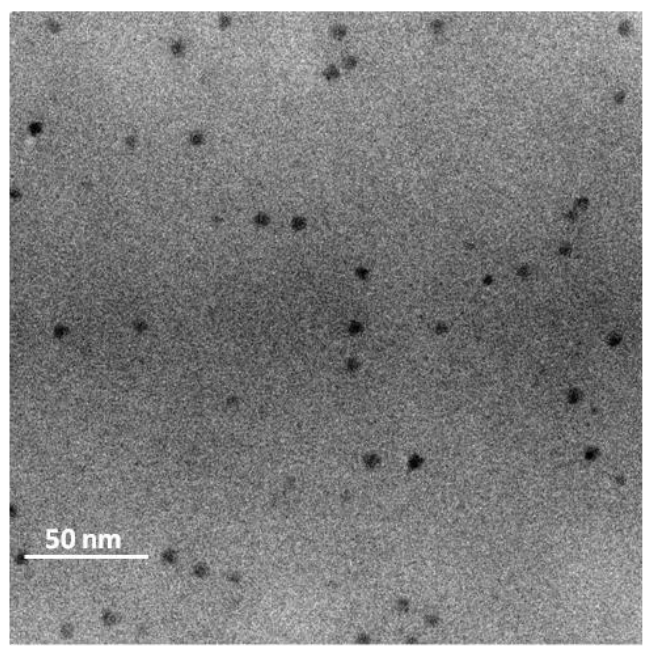

B1

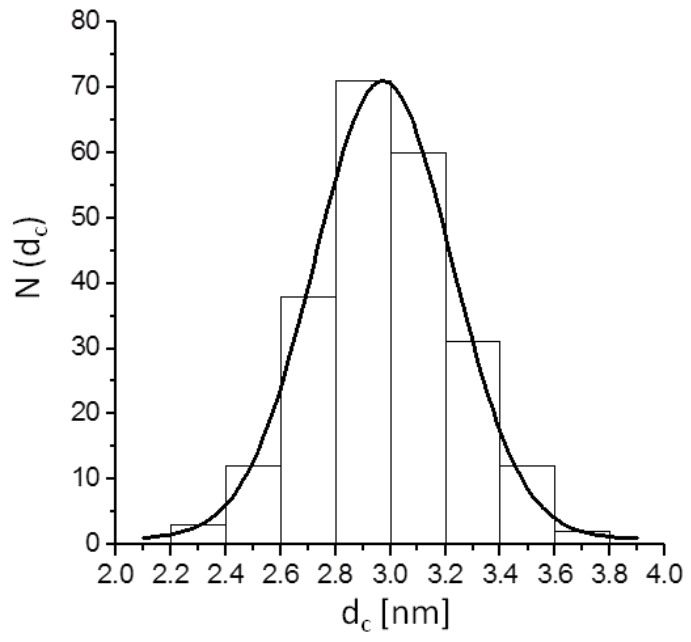

B2

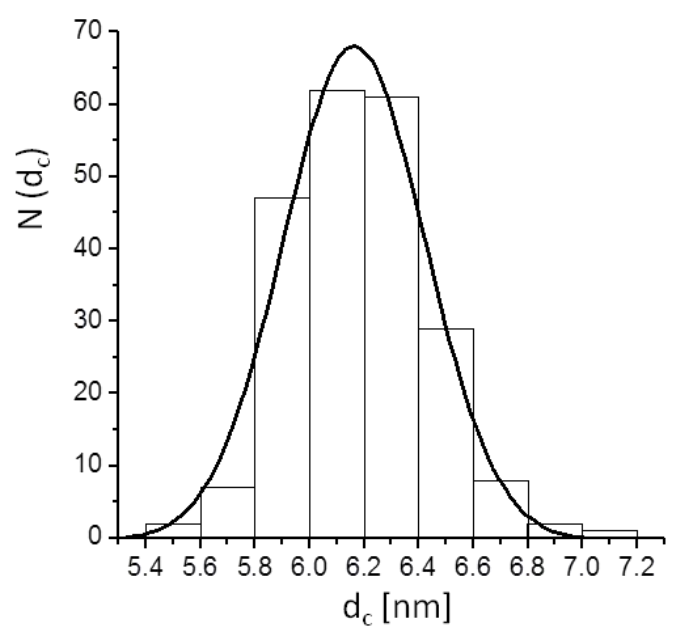

Figure VIII.3.5 A) TEM image of hydrophobic CdSe/ZnS NPs (capped with TOPO, $\approx 4 \mathrm{~nm}$ core diameter, cf. §l.7) dried on grid from a chloroform suspension. The scale bar corresponds to $50 \mathrm{~nm}$. B) Size distribution histogram, plotted as number of $N P s N\left(d_{c}\right)$ that have a core diameter of $d_{c}$. A1 and B1 correspond to CdSe/ZnS NPs of $\approx 3 \mathrm{~nm}$ in diameter. From the histogram in $B 1$, the mean NP diameter and its standard deviation were determined to be $d_{c}=(3.0 \pm 0.2) \mathrm{nm}$. A2 and B2 correspond to CdSe/ZnS NPs of $\approx$ $6 \mathrm{~nm}$ in diameter. From the histogram in B2, the mean NP diameter and its standard deviation were determined to be $d_{c}=(6.2 \pm 0.3) \mathrm{nm}$. 
A

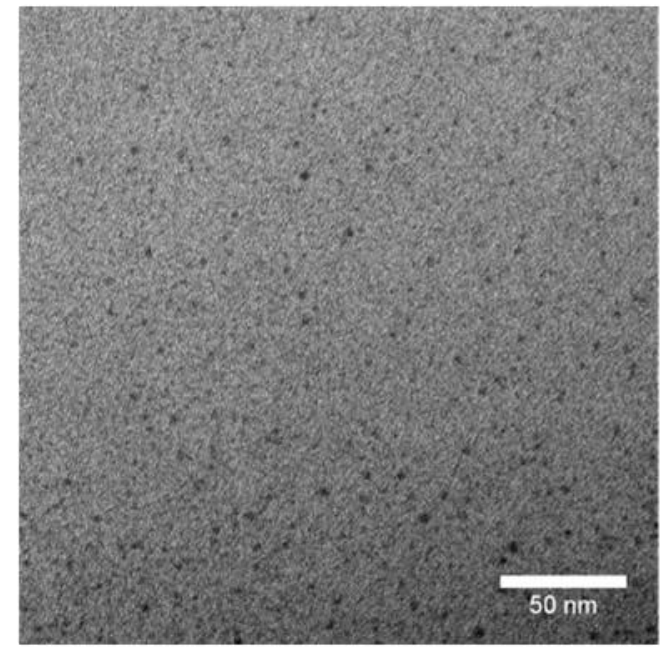

B

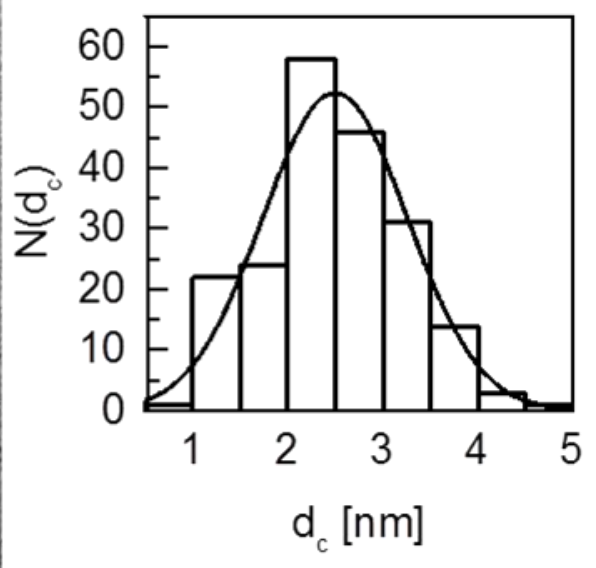

Figure VIII.3.6 A) TEM image of hydrophobic CdS NPS (capped with oleic acid and to some extend with octadecane, $\approx 2 \mathrm{~nm}$ core diameter, $c f . \xi 1.8)$ dried on grid from a chloroform suspension. The scale bar corresponds to $50 \mathrm{~nm}$. B) Size distribution histogram, plotted as number of NPs $N\left(d_{c}\right)$ that have a core diameter of $d_{c}$. From this histogram the mean NP diameter and its standard deviation were determined to be $d_{c}$ $=(2.5 \pm 0.8) \mathrm{nm}$. Note that for NPs of such a very small size, the size measurement as determined by TEM has to be interpreted with care, as NPs with ultra-small size may not provide sufficient contrast and thus are not counted. 
A
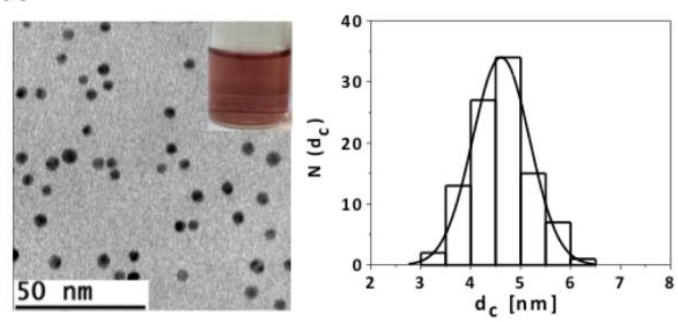

C

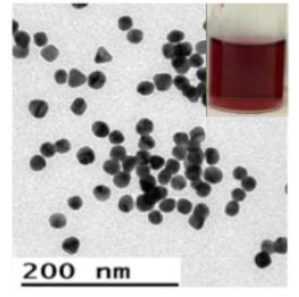

E

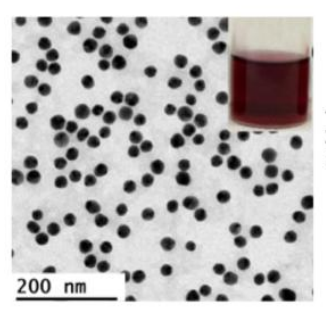

G

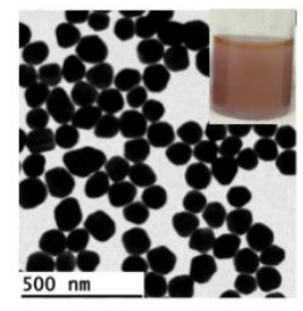

B
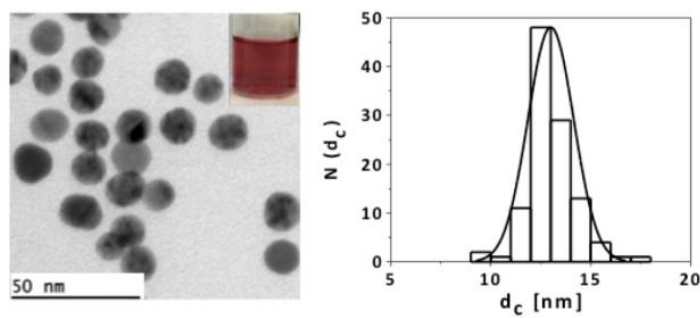

D
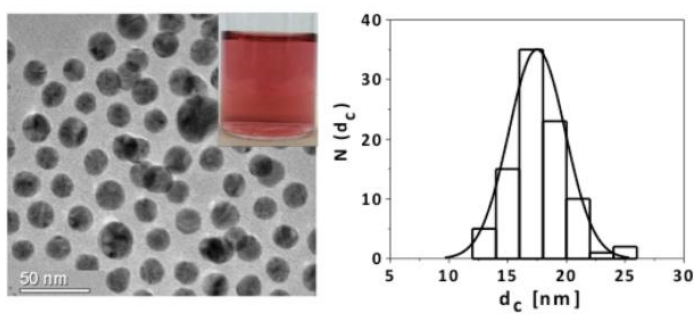

$\mathbf{F}$
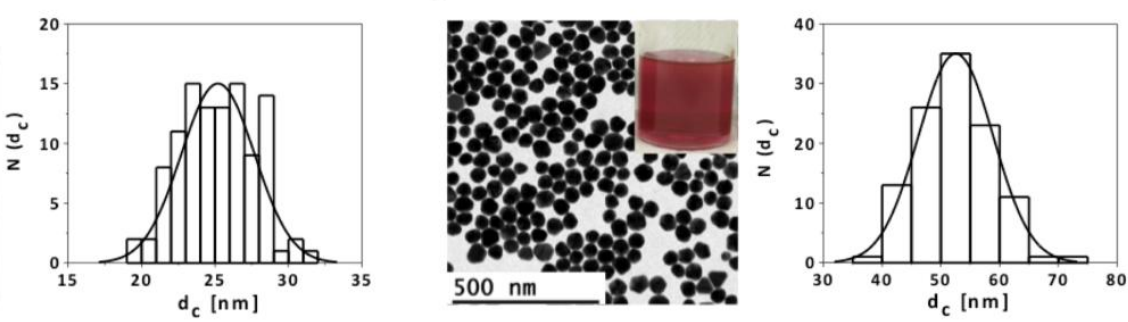

Figure VIII.3.7. TEM images of Cit-Au NPs (cf. \&lI.2) dried on grid from a suspension of NPs in water and their corresponding size distribution histograms, plotted as number of $N P s N\left(d_{c}\right)$ that have a core diameter of $d_{c}$. From these histograms, the mean NP diameter $d_{c}$ and its standard deviation were determined for each preparation. Insets show images of glass vials filled with Cit-Au NPs suspension of each preparation. A) CitAu NPs with $d_{c}=(4.6 \pm 0.5) \mathrm{nm}$, the scale bar corresponds to $50 \mathrm{~nm}$. B) Cit-Au NPs with $d_{c}=(13 \pm 1) \mathrm{nm}$, the scale bars correspond to $50 \mathrm{~nm}$. C) Cit-Au NPs with $d_{c}=(19 \pm 2)$ $\mathrm{nm}$, the scale bar corresponds to $200 \mathrm{~nm}$. D) Cit-Au NPs with $d_{c}=(17 \pm 3) \mathrm{nm}$, the scale bar corresponds to $50 \mathrm{~nm}$. E) Cit-Au NPs, $\approx 25 \mathrm{~nm}$ core diameter, grown on $18 \mathrm{~nm}$ seeds, $d_{c}=(25 \pm 2) \mathrm{nm}$, the scale bar corresponds to $200 \mathrm{~nm}$. F) Cit-Au NPs, with $d_{c}=$ $(53 \pm 6) \mathrm{nm}$, the scale bar corresponds to $500 \mathrm{~nm}$. G) Cit-Au NPs with $d_{c}=(106 \pm 12)$ $\mathrm{nm}$, the scale bar corresponds to $500 \mathrm{~nm}$. 
A

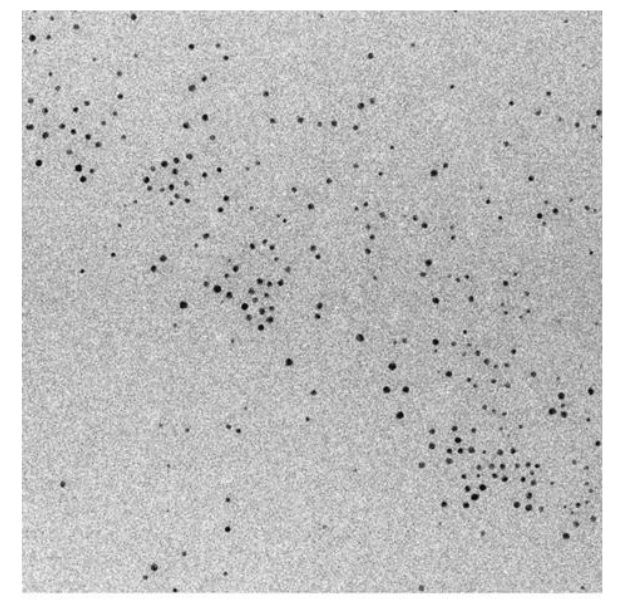

$\underline{200 \mathrm{~nm}}$
B

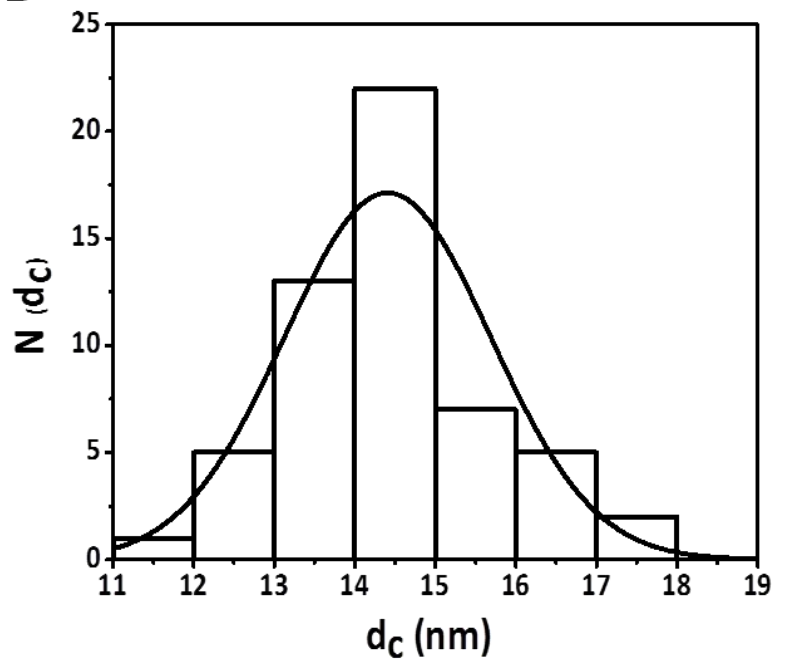

Figure VIII.3.8. A) TEM image of Cit-Ag NPs (cf. §ll.3) dried on grid from a suspension of NPs in water. The scale bar corresponds to $200 \mathrm{~nm}$. B) Size distribution histogram, plotted as number of NPs $N\left(d_{c}\right)$ that have a core diameter of $d_{c}$. From this histogram the mean NP diameter $d_{c}$ and its standard deviation were determined to be $d_{c}=(15 \pm$ 1) $n m$. 
A
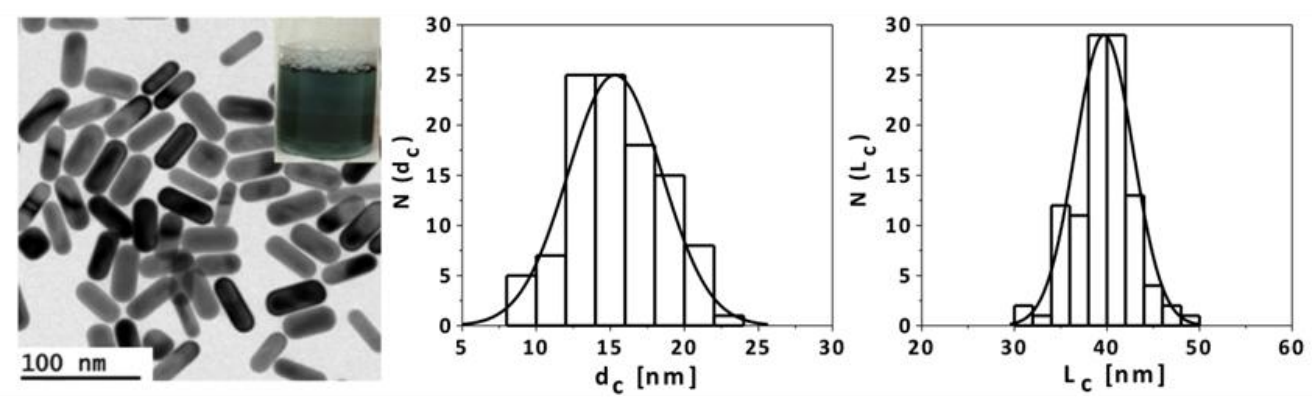

B
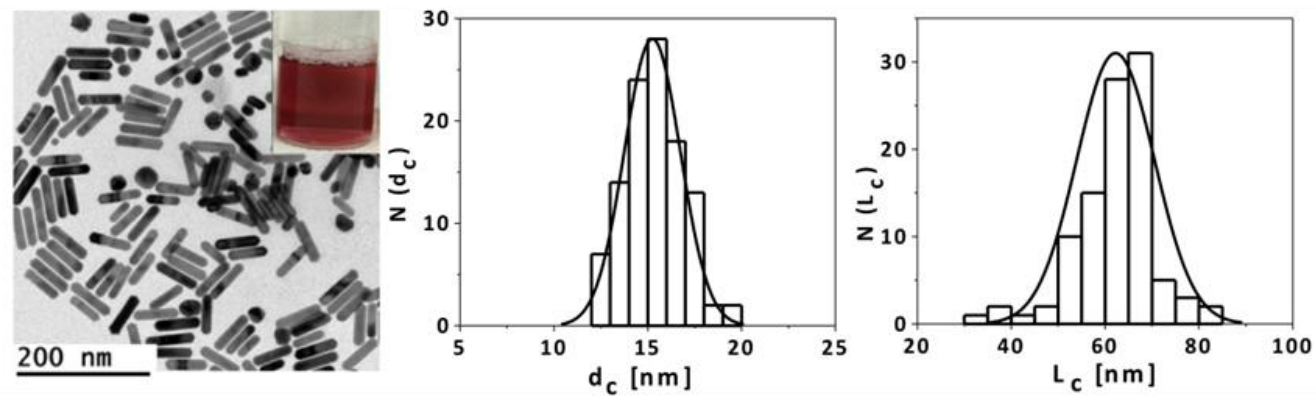

C
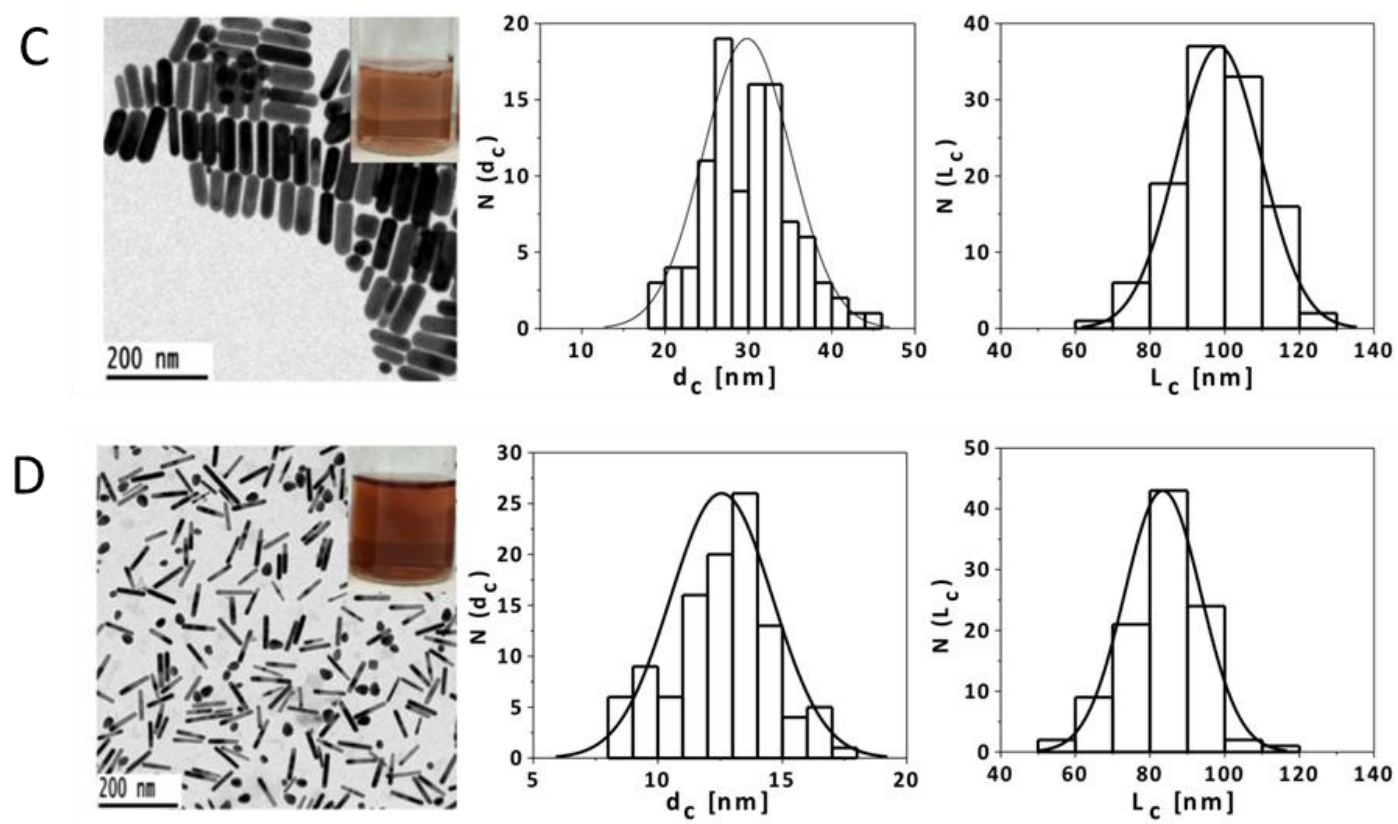

Figure VIII.3.9. TEM images of CTAB-Au NRs (cf. §II.4) dried on grid from a suspension of NPs in water and the corresponding size distribution histograms, plotted as number of $N P s N\left(d_{c}\right)$ that have a core diameter of $d_{c}$ (histograms to the left) or as number of $N P s N\left(L_{c}\right)$ that have a core length of $L_{c}$ (histograms to the right). From these histograms the mean NP diameter $d_{c}$ and its standard deviation, as well as the mean length $L_{c}$ and its standard deviation were determined for each preparation. Insets show images of glass vials filled with CTAB-Au NRs suspension of each preparation. A) $\lambda_{S P R}=650 \mathrm{~nm}, d_{c}$ $=(16 \pm 5) \mathrm{nm}, L_{c}=(40 \pm 3) \mathrm{nm}$, the scale bar corresponds to $100 \mathrm{~nm}$. B) $\lambda_{S P R}=825 \mathrm{~nm}$, $d_{c}=(15 \pm 1) \mathrm{nm}, L_{c}=(62 \pm 8) \mathrm{nm}$, the scale bar corresponds to $\left.200 \mathrm{~nm} . C\right) \lambda_{S P R}=790 \mathrm{~nm}$, $d_{c}=(30 \pm 5) \mathrm{nm}, L_{c}=(98 \pm 11) \mathrm{nm}$, the scale bar corresponds to $200 \mathrm{~nm}$. D) $\lambda_{S P R}=1050$ 
$\mathrm{nm}, d_{c}=(13 \pm 2) \mathrm{nm}, L_{c}=(83 \pm 10) \mathrm{nm}$, the scale bar corresponds to $200 \mathrm{~nm} . \lambda_{S P R}$ corresponds to their surface plasmon peak.

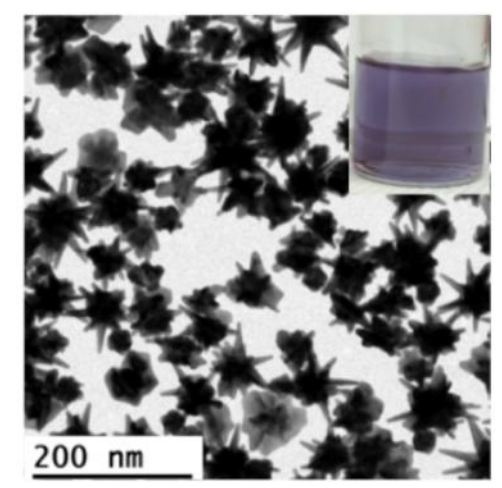

Figure VIII.3.10. TEM image of hydrophilic star-shaped Au NPs (cf. §ll.5) dried on grids from a suspension of NPs in water. The scale bar corresponds to $200 \mathrm{~nm}$. The inset shows a glass vials filled with suspension of star-shaped Au NPs.
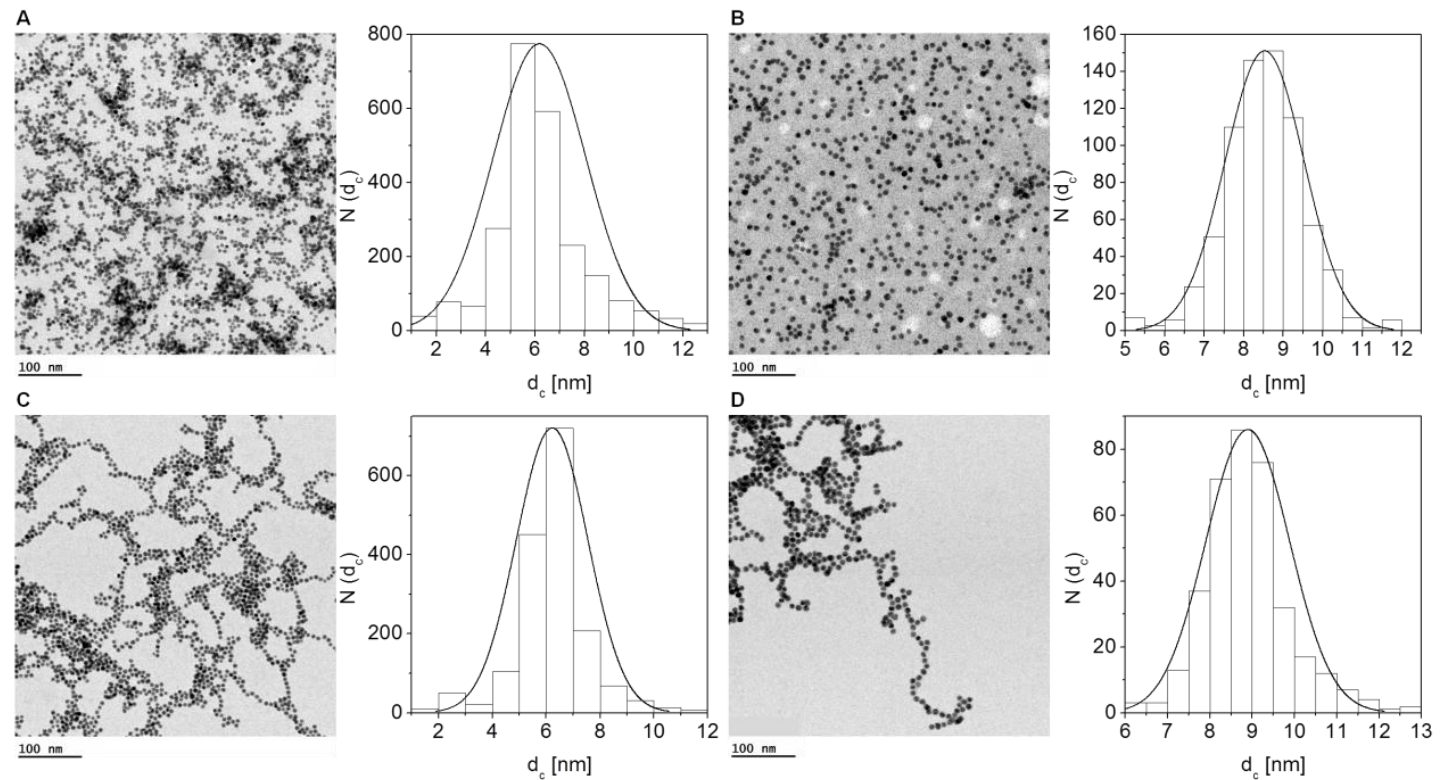

Figure VIII.3.11. TEM images of Cit-Au NPs (cf. §ll.2) dried on grid from a suspension of $N P s$ in water and their corresponding size distribution histograms before (A\&C) and after (B\&D) ligand exchange with bis(p-sulfonatophenyl)-phenylphosphine (cf. §III.2). Histograms are plotted as number $N\left(d_{c}\right)$ of NPs which have a core diameter $d_{c}$. From these histograms the mean NP diameter $d_{c}$ and its standard deviation were determined. All scale bars correspond to $100 \mathrm{~nm}$. A) Cit-Au NPs before ligand exchange with $d_{c}=(6.0 \pm 1.1) \mathrm{nm}$. B) Au NPs after exchanging the original citrate capping ions with bis(p-sulfonatophenyl)-phenylphosphine with, $d_{c}=(8.7 \pm 1.0) \mathrm{nm}$. C) Cit-Au NPs before ligand exchange with $d_{c}=(6.3 \pm 0.8) \mathrm{nm}$. E) Au NPs after exchanging the original 
citrate capping ions with bis(p-sulfonatophenyl)-phenylphosphine with $d_{c}=(8.9 \pm 0.8)$ $n m$.
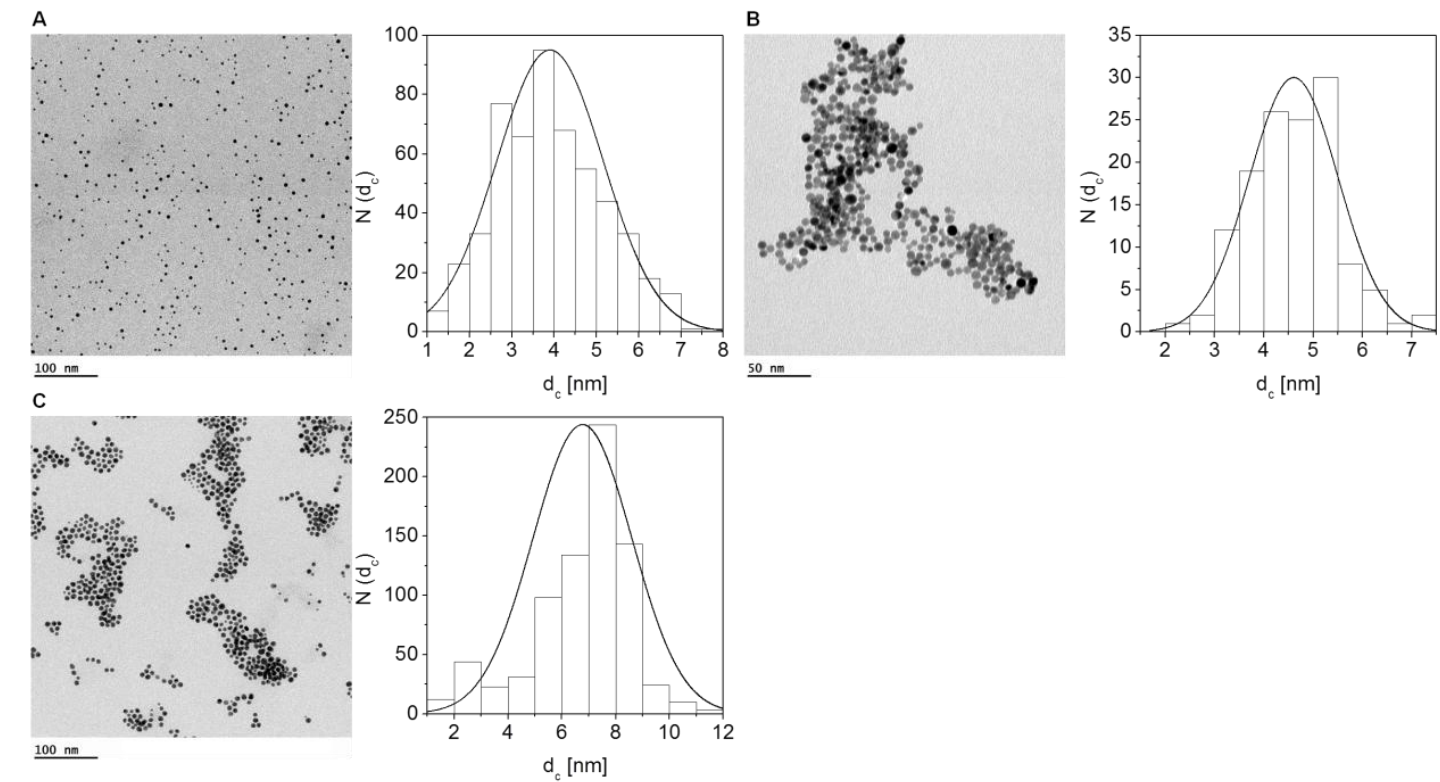

Figure VIII.3.12. TEM images of PMA-Au NPs, made by DDT-Au NPs (cf. §l.2), which have been transferred to aqueous solution using PMA-g-dodecyl polymer coating or ligand exchange method (cf. $\S V I .3$ and $\S V .2$, respectively) and dried on grid from a suspension of NPs in water, along with corresponding size distribution histograms. Histograms are plotted as number of NPs $N\left(d_{c}\right)$ that have a core diameter $d_{c}$ to calculate the mean NP diameter $d_{c}$ and its standard deviation. A) PMA-Au NPs (DDT-Au NPs after coating with PMA-g-dodecyl polymer, SVI.3) with $d_{c}=(4.1 \pm 1.1) \mathrm{nm}$, the scale bar corresponds to $100 \mathrm{~nm}$. B) Au NPs originally capped with hexanoic acid, $\approx 4$ $\mathrm{nm}$ core diameter, after ligand exchange with mercaptopropionic acid (cf. $\S V .2$ ), $d_{c}=$ $(4.4 \pm 0.9) \mathrm{nm}$, the scale bar corresponds to $50 \mathrm{~nm}$. C) Au NPs originally capped with decanoic acid, $\approx 7 \mathrm{~nm}$ core diameter, after ligand exchange with mercaptoundecanonic acid (cf. $\S V .2), d_{c}=(7.1 \pm 1.3) \mathrm{nm}$, the scale bar corresponds to $100 \mathrm{~nm}$. 

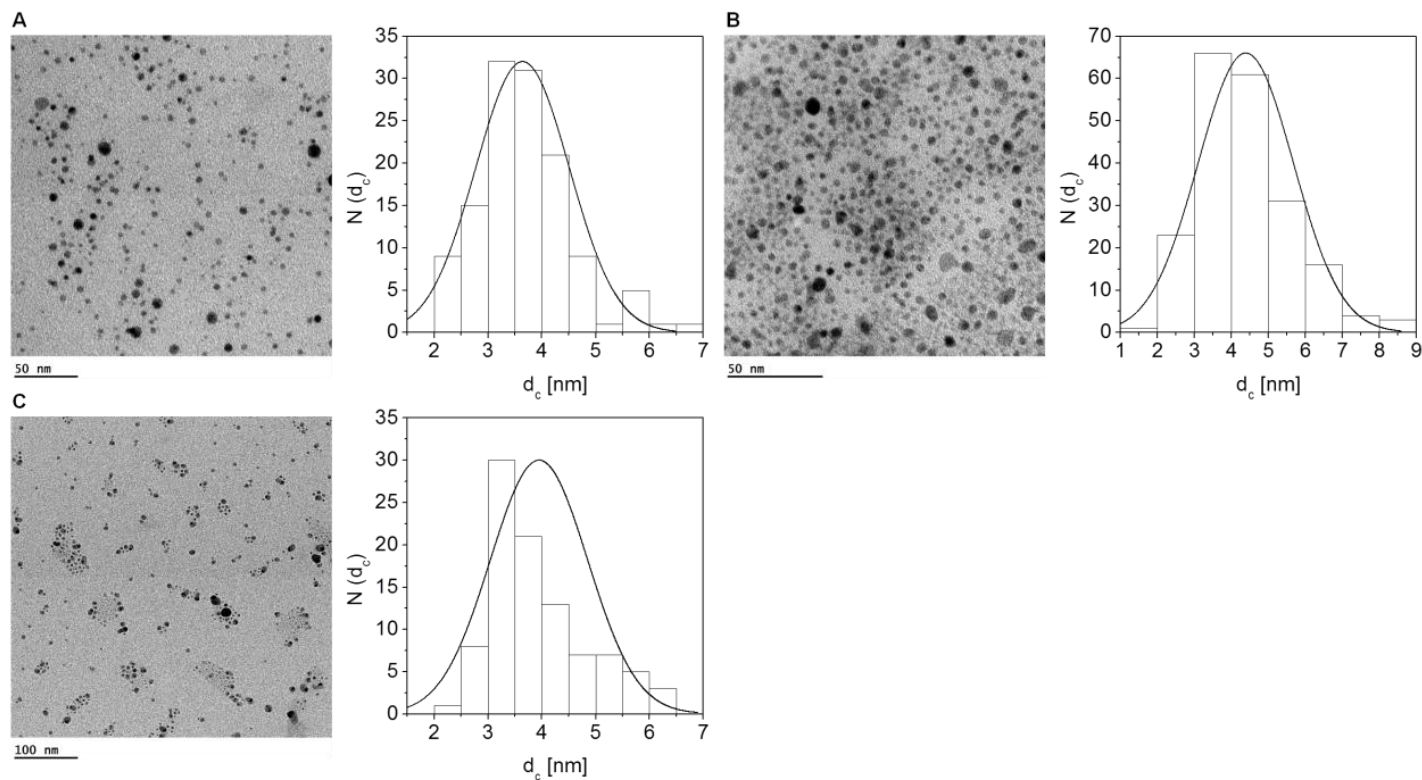

Figure VIII.3.13. TEM images of hydrophobic Ag NPs originally capped with dodecanethiol of $\approx 4 \mathrm{~nm}$ core diameter (cf. §l.3), which have been transferred to aqueous solution using PMA-g-dodecyl polymer coating or the ligand exchange method (cf. §VI.3 and $\S V .2$, respectively) and dried on grid from a suspension of NPs in water. Histograms are plotted as number of $N P s N\left(d_{c}\right)$ that have a core diameter $d_{c}$ to calculate the mean NP diameter $d_{c}$ and its standard deviation. A) PMA-Ag NPs (DDT-Ag NPs after coating with PMA-g-dodecyl polymer, $\S V I .3)$ with $d_{c}=(3.6 \pm 0.9) \mathrm{nm}$, the scale bar corresponds to $50 \mathrm{~nm}$. B) Ag NPs after ligand exchange with mercaptopropionic acid (cf. $\S V .3), d_{c}=(4.4 \pm 1.3) \mathrm{nm}$, the scale bar corresponds to 50 $n m$. C) Ag NPs after ligand exchange with mercaptoundecanonic acid (cf. $\S V .3$ ), $d_{c}=$ $(4.4 \pm 1.3) \mathrm{nm}$, the scale bar corresponds to $100 \mathrm{~nm}$.

\section{VIII.4 Transmission electron microscopy of NPs including their organic shell}

As shown in the previous sections, TEM imaging is used to determine the size $\left(d_{c}\right)$ and shape (rods, spheres, stars, etc.) of NP cores composed of various materials such as $\mathrm{Au}$, $\mathrm{Ag}, \mathrm{Fe}_{3} \mathrm{O}_{4}, \mathrm{FePt}$, etc. The inorganic cores of the NPs appear almost black due to the strong electron-scattering character of these materials. However, as it has been previously discussed, these NPs are modified with different organic ligands, which increase the effective diameter of the NPs (e.g., $\mathrm{d}_{\text {eff }}$ ). Such organic shells have low electron scattering character, and thus do not show sufficient contrast to be visible in regular TEM images. In order to investigate the thickness of these organic coatings (e.g., $I_{\text {ligand }}$ ), negative staining is needed. Heavily electron-scattering compounds (e.g. uranyl acetate, ammonium molibdate, or osmium tetroxide) may be used to stain the 
background of TEM grids and indirectly allow the visualization of the organic shell around NPs ${ }^{93}$. Ideally, negative staining contrast agents form a homogeneous dark background and thus the organic shells around NPs appear as a bright corona (i.e., negative staining) around the dark inorganic cores (cf. Figure VIII.4.1.)

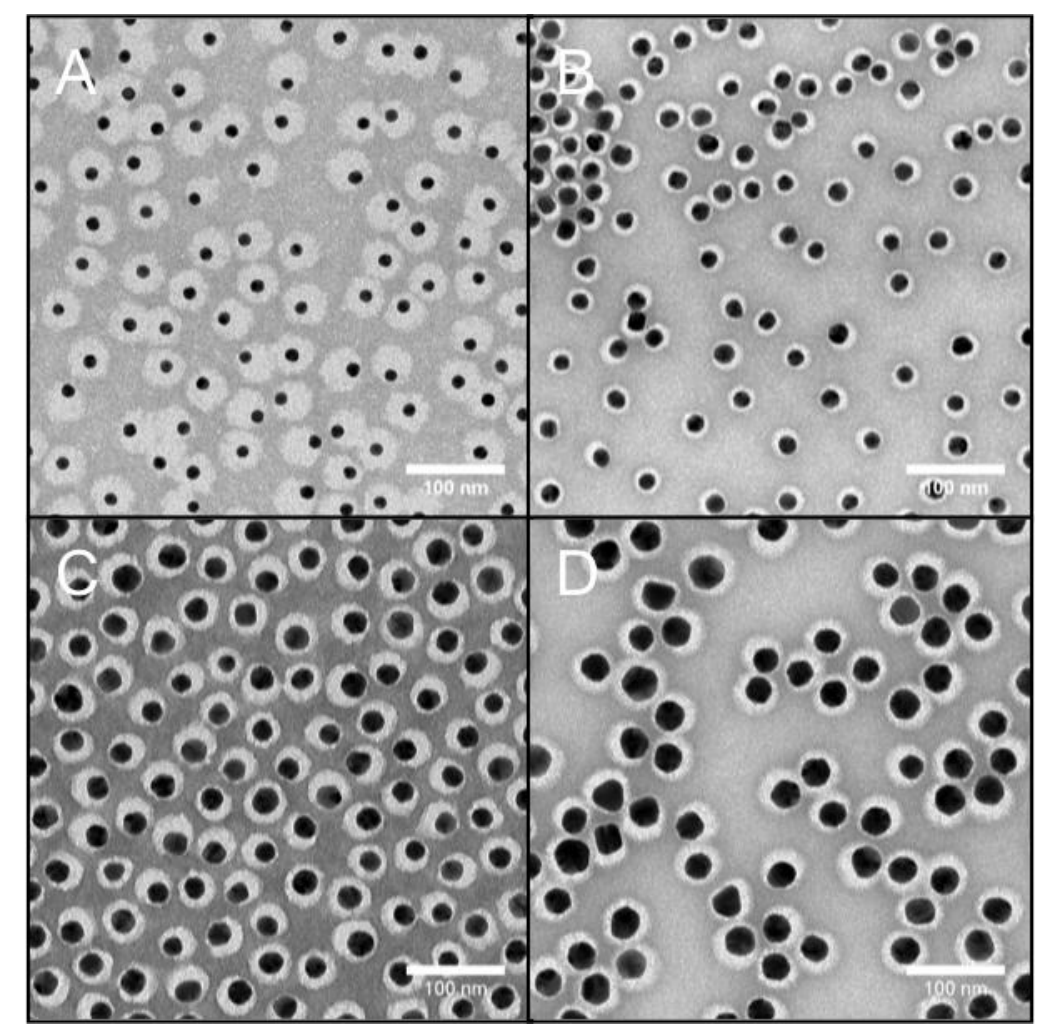

Figure VIII.4.1. Visualizing the PEG shell around PEG-Au NPS using TEM imaging and the negative staining technique with uranyl acetate ${ }^{55}$ (cf. §ll.2 and §III.3). A) Au cores $\left(d_{c}=13.7 \pm 0.7 \mathrm{~nm}\right)$ plus an organic shell of PEG $(10 \mathrm{kDa}$, I ligand $=11.8 \pm 1.2 \mathrm{~nm})$ results in $d_{\text {eff }}=37.4 \pm 2.5 \mathrm{~nm}$. B) Au cores $\left(d_{c}=18.9 \pm 1.9 \mathrm{~nm}\right)$ plus an organic shell of PEG (1 $\left.k D a, l_{\text {ligand }}=3.2 \pm 0.4 \mathrm{~nm}\right)$ results in $d_{\text {eff }}=25.4 \pm 2.1 \mathrm{~nm}$. C) Au cores $\left(d_{c}=23.5 \pm 1.7 \mathrm{~nm}\right)$ plus an organic shell of PEG (5 kDa, l ligand $=11.8 \pm 1.5 \mathrm{~nm})$ results in $d_{\text {eff }}=37.4 \pm 1.5 \mathrm{~nm}$. D) Au cores $\left(d_{c}=30.1 \pm 2.6 \mathrm{~nm}\right)$ plus an organic shell of PEG $\left(3 \mathrm{kDa}\right.$, $l_{\text {ligand }}=7.7 \pm 1.0$ $\mathrm{nm}$ ) results in $d_{\text {eff }}=45.6 \pm 3.6 \mathrm{~nm}$. Mean and corresponding standard deviation values of the core $\left(d_{c}\right)$, ligand shell (l ligand), and the effective size $\left(d_{\text {eff }}\right)$, were obtained by analyzing several negative staining TEM micrographs (> 1000 NPs) as described by del Pino et $a l^{55}$. TEM images, such as shown in Figure VIII.4.1, were segmented using Matlab (Mathworks) and Cellprofiler ${ }^{94}$. The scale bars are $100 \mathrm{~nm}$.

The technique can be visualized as to balance the competition between the sample and the staining agent to reach the grid surface. Therefore, for a successful negative staining it may be important to take into account several sample parameters as for example the overall particle charge or its diffusion rate together of such of the chosen 
stain. This translates into the need of different approaches to achieve homogenous negative contrast for different samples. For PEGylated Au NPs (cf. §II.2 and §III.3), as shown in Figure VIII.4.1, a sample preparation protocol reported by Harris ${ }^{93}$ was optimized as follows ${ }^{55}$ : Samples were prepared on carbon film 400 copper mesh grids purchased from Electron Microscopy Sciences (Hatfield, USA). To enhance the hydrophilicity of grids and to impart them with negative charge, the specimen grids were exposed to glow-discharge treatment under air plasma for $20 \mathrm{~s}\left(2.0 \times 10^{-1} \mathrm{~atm}\right.$ and $30 \mathrm{~mA}$ ), using a MED 020 modular high vacuum coating system (BAL-TEC AG, Balzers, Fürstentum Liechtenstein). Negatively charged carbon grids were used within 5 min after treatment to ensure hydrophilicity. $0.35 \mu \mathrm{L}$ sample droplet of NP solution with NP concentration ranging from $10 \mathrm{nM}$ (for Au cores of $14 \mathrm{~nm}$ diameter) to $1.5 \mathrm{nM}$ (for Au cores of $30 \mathrm{~nm}$ diameter) of PEGylated Au NPs was placed on the grid, allowed to incubate for $20 \mathrm{~s}$ to $1 \mathrm{~min}$ (the smaller the particle size, the lower the incubation time) followed by $0.35 \mu \mathrm{L}$ droplet of $0.25 \%$ weight/volume $(\mathrm{w} / \mathrm{v})$ uranyl acetate aqueous solution. The grid was air-dried overnight before actual TEM imaging.

\section{VIII.5 X-ray diffraction}

With X-ray diffraction (XRD) the diameter $d_{\text {crys }}$ of the crystalline part of the NP core can be determined. For this, a diffractogram has to be recorded. Briefly, and in most of the cases, a NP powder solid sample is irradiated with monochromatic $\mathrm{X}$-ray radiation, and the diffraction (i.e. the change of the direction of the elastically scattered radiation) is recorded. For a crystalline material, diffraction occurs only for several angles, named Bragg's angles $(\Theta)$. Such angles are associated with crystallographic planes, which depend on the unit cell parameter of analyzed material. In a typical XRD experiment, the intensity (i.e. number of counts in the detector for a determined $\Theta$ ) is plotted versus the double of the diffraction angle $(2 \Theta)$. The number and position of peaks in a powder diffractogram can be used for phase identification, since every individual crystalline compound has its own "fingerprint", which is determined by its crystallographic structure. The intensity of the peaks (i.e. the number of counts in the detector for a determined $2 \Theta$ ) is related to the atomic positions in the crystalline unit cell, and the width of every peak is connected with the crystallite size and microstrain in the corresponding crystallographic direction.

The crystallite size for a crystalline direction, determined by a crystallographic plane and a Bragg's angle $(\Theta)$, can be estimated with the Scherrer equation:

$$
\mathrm{d}_{\text {crys }}=\frac{\mathrm{K} \lambda}{\beta \cos \Theta}
$$


where $d_{\text {crys }}$ is the crystallite size. $K$ is the shape factor or Scherrer constant, which varies in the range $0.89<\mathrm{K}<1$, and usually $\mathrm{K}=0.9$. $\lambda$ is the wavelength of the $\mathrm{X}$-ray ( for $\mathrm{Cu} \mathrm{K}$ - alpha $=0.15418 \mathrm{~nm}$ ). $\beta$ is the line broadening at half the maximum intensity (FWHM). $\Theta$ is the Bragg's angle of the analyzed peak.

For single crystal NPs, the crystallite size $d_{\text {crys }}$ estimated by the Scherrer equation is expected to be very similar to the geometric particle size $d_{c}$ as determined by other characterization techniques such as TEM ${ }^{95}$. In case of polycrystalline NPs, the obtained crystalline sizes are much smaller than the actual NP size.

Here, X-ray diffraction (XRD) studies were carried out using a Panalytical X'Pert Pro diffractometer equipped with an X-Celerator detector. The NPs were deposited on a silicon sample holder. In this instrument a $\Theta: \Theta$ Bragg-Brentano geometry is used, in which the sample is fixed, the tube rotates at a rate $\Theta^{\circ} / \mathrm{min}$, and the detector rotates at the same rate of rate of $\Theta^{\circ} / \mathrm{min}$. In Figure VIII.5.1 data obtained with dodecanethiolcapped Au NPs ( $\approx 4 \mathrm{~nm}$ core diameter, $c f$. §I.2) overcoated with PMA (cf. §VI.3) are shown. The XRD pattern indicates that the analyzed NPs consisted of $f c c$ Au crystals. (PDF card number: 00-004-0784).

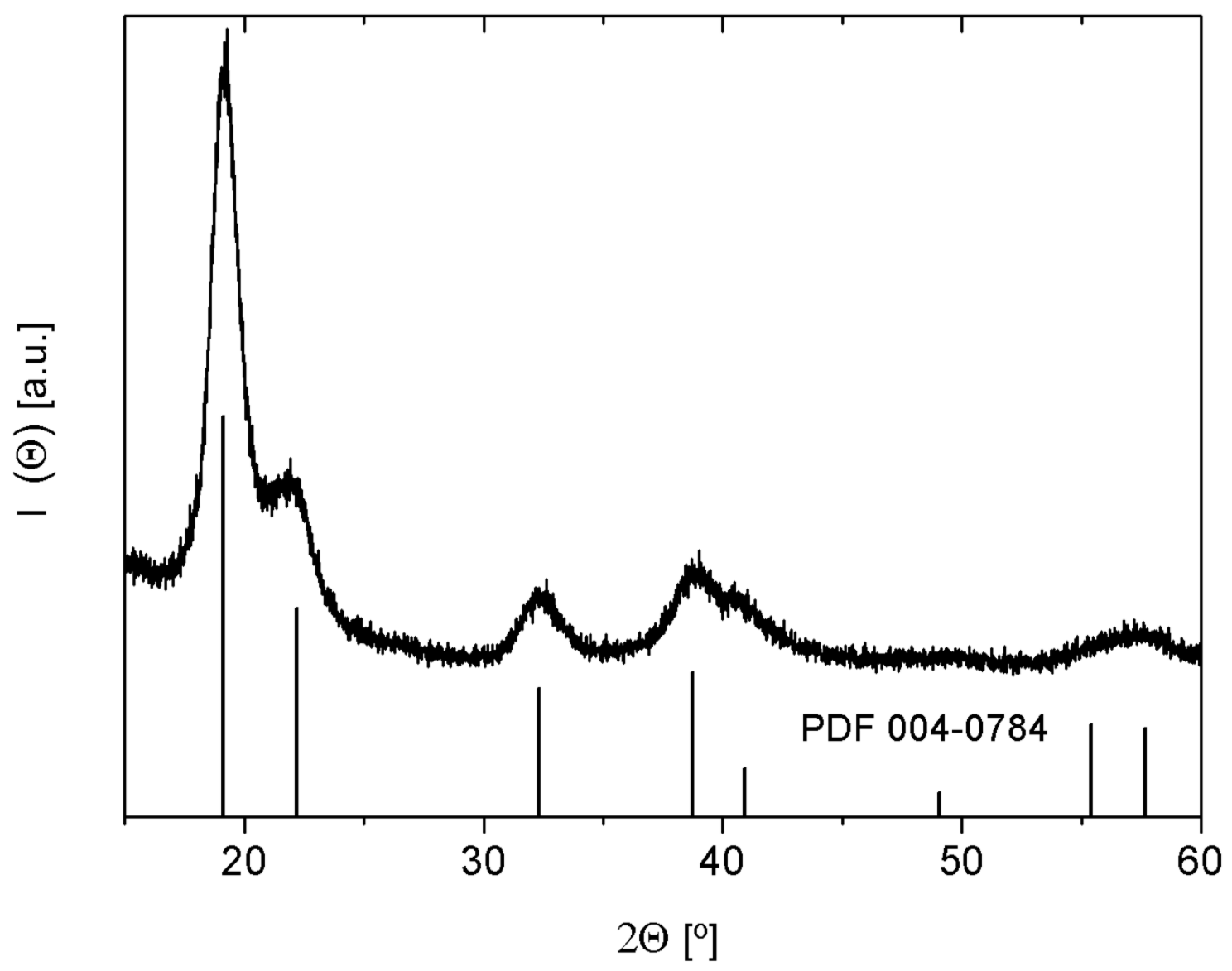


Figure VIII.5.1. XRD diagram I( $\Theta)$ of polymer coated Au NPs $(\approx 4 \mathrm{~nm}$ core size, $c f$. \&l.2 and §VI.3). The PDF pattern of cubic gold has also been included.

The peak corresponding to the (111) plane $\left(2 \Theta\right.$ around $\left.38.5^{\circ}\right)$ was used to determine the crystallite size. It should be taken into account that $\Theta$ values (and not $2 \Theta$ ), should be used, as well as radians units (and not degrees), especially for the $\beta$ (FWHM) value. The first step consisted of the appropriate plot of the desired peak (Intensity I versus $\Theta)$. The determination of the full width at half maximum (FWHM) value was carried out by fitting the peak. In most of the cases a GauSamp function provides accurate results (cf. Figure VIII.5.2).

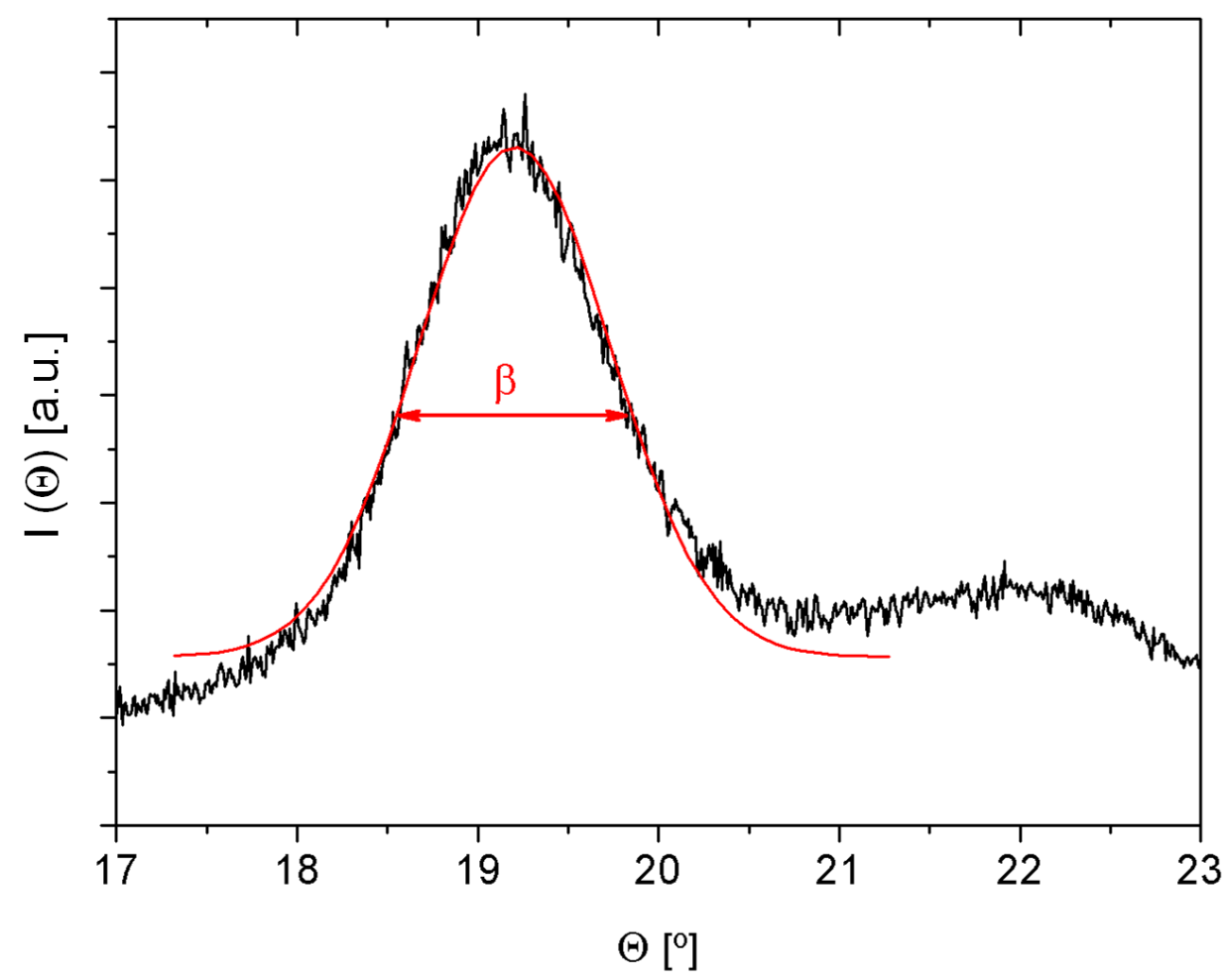

Figure VIII.5.2. XRD diagram I( $\Theta)$ of the (111) plane of polymer coated Au NPs $(\approx 4 \mathrm{~nm}$ core size, cf. \$I.2 and \$VI.3). The red line represents the GauSamp fit of the peak. This graph is a part of the graph shown in Figure VIII.5.1.

In the presented case, the following values were obtained: $\Theta=19.198^{\circ}$; FWHM $=$ $1.457^{\circ}=0.0255 \mathrm{rad}$, and thus according to Equation VIII.5.1: $\mathrm{d}_{\text {crys }}=(\mathrm{K} \times \lambda) /(\beta \cdot \cos (\Theta))=$ $(0.9 \times 0.15418 \mathrm{~nm}) /\left(0.0255 \times \cos \left(19.198^{\circ}\right)\right)=5.7 \mathrm{~nm}$. In this way the crystallite size determined from the width of the (111) peak was $d_{\text {crys }}=5.7 \pm 0.2 \mathrm{~nm}$, in acceptable agreement with the TEM data (cf. Figure VIII.3.1, the actually determined value for the batch used here was $\left.d_{c}=4.3 \mathrm{~nm}\right)$. 


\section{VIII.6 Thermogravimetric analysis of NPs}

Thermogravimetric analysis (TGA) measures the mass of a sample, while its temperature is continuously increased. Upon decomposition of parts of the sample its mass decreases and thus allows for analyzing the composition of the sample. In the case of inorganic NPs upon heating the solvent may be evaporated, as the organic shell around the NPs surface may decompose. In this section, calculation of the weight contribution of the organic coating on NPs to the total weight of the NPs will be detailed.

In Figure VIII.6.1A the TGA analysis carried out on DDT-Au NPs $(\approx 4 \mathrm{~nm}$ core diameter, cf. §I.2) is shown. A suspension of the hydrophobic NPs in chloroform was added dropwise to an alumina sample holder, and the solvent was evaporated in an oven at $40{ }^{\circ} \mathrm{C}$. The process was repeated until around $\mathrm{m}_{\mathrm{NP} \_ \text {pel }} \approx 2 \mathrm{mg}$ of NPs were deposited on the sample holder. TGA was performed in air at a heating rate of $5^{\circ} \mathrm{C} \cdot \mathrm{min}^{-1}$, using a Q600 TA Instrument.

The TGA curve corresponding to the dodecanethiol-capped Au NPs (Figure VIII.6.1A) showed a very slight loss $(<1 \%)$ in the $25-150{ }^{\circ} \mathrm{C}$ range, as expected from hydrophobic NPs. An abrupt loss (around 18\%) took place between 150 and $300{ }^{\circ} \mathrm{C}$, which should be caused by the decomposition of the DDT. No changes in the mass were observed at higher temperatures. Based on these data, an estimation of the

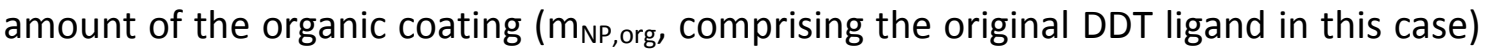
deposited on the NPs surface can be determined. The diameter of the inorganic Au core of this batch of Au NPs had been determined to be $d_{c}=4.3 \mathrm{~nm}$. The mass $m_{N P}$ of one NP comprises the mass of the inorganic core $m_{c}$ and the mass of the organic coating $\mathrm{m}_{\mathrm{NP}, \mathrm{org}}$ :

$$
\mathrm{m}_{\mathrm{NP}}=\mathrm{m}_{\mathrm{c}}+\mathrm{m}_{\mathrm{NP}, \mathrm{org}}
$$

In the present case according to Equation VIII.1.1 and Equation VIII.1.4 the volume $\mathrm{V}_{c}$ and the mass $m_{c}$ of one NP core could be calculated. For a spherical Au NP with core diameter $\mathrm{d}_{\mathrm{c}}=4.3 \mathrm{~nm}, \mathrm{~V}_{\mathrm{c}}=(\pi / 6) \times(4.3 \mathrm{~nm})^{3} \approx 41.6 \mathrm{~nm}^{3}$. Given the bulk density of Au of $\rho_{\text {Au }}=19.3 \mathrm{~g} / \mathrm{cm}^{3}$, the mass of one Au core thus is $m_{c}=19.3 \mathrm{~g} / \mathrm{cm}^{3} \times 41.6 \mathrm{~nm}^{3} \approx 803 \mathrm{~g}$ $\times\left(10^{-9} \mathrm{~m} / 10^{-2} \mathrm{~m}\right)^{3} \approx 8.0 \times 10^{-19} \mathrm{~g}(c f . \S \mathrm{VIII} 1)$.

In the data shown in Figure VIII.6.1A, the absolute weights for the NP pellet ( $\mathrm{m}_{\mathrm{NP} \_ \text {pel }}(T)$ ) were: $\mathrm{m}_{\mathrm{NP} \_ \text {pel }}\left(25^{\circ} \mathrm{C}\right)=1.744 \mathrm{mg}$ (mass at RT), $\mathrm{m}_{\mathrm{NP} \_ \text {pel }}\left(150^{\circ} \mathrm{C}\right)=1.730 \mathrm{mg}, \mathrm{m}_{\mathrm{NP} \_ \text {pel }}(340$ $\left.{ }^{\circ} \mathrm{C}\right)=1.424 \mathrm{mg}$, and $\mathrm{m}_{\mathrm{NP} \_ \text {pel }}\left(70{ }^{\circ} \mathrm{C}\right)=1.421 \mathrm{mg}$ (final mass). We considered that at 700 
${ }^{\circ} \mathrm{C}$ only the NP cores were present. The number of cores (and thus of NPs) in the sample thus was $\mathrm{N}_{\mathrm{c}}=\mathrm{m}_{\mathrm{NP} \_ \text {pel }}\left(700{ }^{\circ} \mathrm{C}\right) / \mathrm{m}_{\mathrm{c}}=1.421 \times 10^{-3} \mathrm{~g} / 8.0 \times 10^{-19} \mathrm{~g}=1.77 \times 10^{15} \approx$ $1.8 \times 10^{15}$. We attributed the mass of the organic coating (DDT) to the mass lost upon heating from $150{ }^{\circ} \mathrm{C}$ to $340{ }^{\circ} \mathrm{C}: \mathrm{m}_{\mathrm{NP} \text {,org_pel }}=\mathrm{m}_{\mathrm{NP} \_ \text {pel }}\left(150^{\circ} \mathrm{C}\right)-\mathrm{m}_{\mathrm{NP} \_ \text {pel }}\left(340{ }^{\circ} \mathrm{C}\right)=1.730 \mathrm{mg}$ $-1.424 \mathrm{mg}=0.306 \mathrm{mg}$. Thus, the mass of the organic coating around one NP is $\mathrm{m}_{\mathrm{NP} \text {, org }}$ $=\mathrm{m}_{\mathrm{NP} \text {,org_pel }} / \mathrm{N}_{\mathrm{c}}=0.306 \times 10^{-3} \mathrm{~g} / 1.8 \times 10^{15} \approx 1.7 \times 10^{-19} \mathrm{~g}$. Therefore, the whole mass of one Au NP is according to Equation VIII.6.1 $\mathrm{m}_{\mathrm{NP}}=\mathrm{m}_{\mathrm{c}}+\mathrm{m}_{\mathrm{NP}, \text { org }}=8.0 \times 10^{-19} \mathrm{~g}+1.7 \times 10^{-19} \mathrm{~g}$ $\approx 9.7 \times 10^{-19} \mathrm{~g}$. The organic coating thus contributed $\mathrm{m}_{\mathrm{NP}, \mathrm{org}} / \mathrm{m}_{\mathrm{NP}}=1.7 \times 10^{-19} \mathrm{~g} / 9.7 \times 10^{-19}$ $\mathrm{g} \approx 17 \%$ of the mass of one NP.

The TGA analysis of the DDT-Au NPs overcoated with PMA ( $c f . \S \mathrm{VI.3)}$ is shown in Figure VIII.6.1B. The PMA-Au NPs, which were suspended in water, were cleaned by ultracentrifugation ( $c f$. §VII.2). In this case, the sample was dried before analysis, and around $\mathrm{m}_{\mathrm{NP} \_ \text {pel }} \approx 1 \mathrm{mg}$ of pelleted PMA coated NPs were placed in an alumina sample holder. TGA was also performed in air at a heating rate of $5{ }^{\circ} \mathrm{C} \cdot \mathrm{min}^{-1}$, using the same Q600 TA Instrument.

The TGA curve of the DDT-Au NPs overcoated with PMA showed a slight weight loss (around $5 \%$ ) in the $25-150{ }^{\circ} \mathrm{C}$ range, which can be ascribed to the release of absorbed water. An additional abrupt loss (around 26\%) took place between 150 and $300{ }^{\circ} \mathrm{C}$, which should be caused by the decomposition of the dodecanethiol and/or the amphiphilic polymer poly(isobutylene-alt-maleic anhydride)-graft-dodecyl (PMA). Almost no changes in the mass were observed at higher temperatures. As in the previous hydrophobic sample, the TGA data could be used to estimate the mass of organic coating $\mathrm{m}_{\mathrm{NP} \text {, org }}$ of one Au NP. The mass of an Au NP core was calculated as described above to be $8.0 \times 10^{-19} \mathrm{~g}$.

The absolute weights $\mathrm{m}_{\mathrm{NP} \_ \text {pel }}(\mathrm{T})$ in Figure VIII.6.1B were: $\mathrm{m}_{\mathrm{NP} \_ \text {pel }}\left(25^{\circ} \mathrm{C}\right)=1.032 \mathrm{mg}$ (mass at RT), $\mathrm{m}_{\text {NP_pel }}\left(150^{\circ} \mathrm{C}\right)=0.990 \mathrm{mg}, \mathrm{m}_{\mathrm{NP} \_ \text {pel }}\left(340^{\circ} \mathrm{C}\right)=0.711 \mathrm{mg}, \mathrm{m}_{\mathrm{NP} \_ \text {pel }}\left(700^{\circ} \mathrm{C}\right)=$ $0.693 \mathrm{mg}$ (final mass). As in the previous sample, we considered that at $700{ }^{\circ} \mathrm{C}$ only the NP cores were present. The number of cores (and thus of NPs) in the sample thus was $\mathrm{N}_{\mathrm{c}}=\mathrm{m}_{\mathrm{NP} \_ \text {pel }}\left(700{ }^{\circ} \mathrm{C}\right) / \mathrm{m}_{\mathrm{c}}=0.693 \times 10^{-3} \mathrm{~g} / 8.0 \times 10^{-19} \mathrm{~g}=8.66 \times 10^{14} \approx 8.7 \times 10^{14}$. We attributed the mass of the organic coating to the mass lost upon heating from $150{ }^{\circ} \mathrm{C}$ to $340{ }^{\circ} \mathrm{C}: \mathrm{m}_{\mathrm{NP} \text {,org_pel }}=\mathrm{m}_{\mathrm{NP} \_ \text {pel }}\left(150^{\circ} \mathrm{C}\right)-\mathrm{m}_{\mathrm{NP} \_ \text {pel }}\left(340{ }^{\circ} \mathrm{C}\right)=0.990 \mathrm{mg}-0.711 \mathrm{mg}=0.279$ $\mathrm{mg}$. Thus, the mass of the organic coating around one NP was $m_{N P, \text { org }}=m_{N P \text {,org_pel }} / N_{c}=$ $0.279 \times 10^{-3} \mathrm{~g} / 8.7 \times 10^{14}=3.21 \times 10^{-19} \mathrm{~g} \approx 3.2 \times 10^{-19} \mathrm{~g}$. Thus the whole mass of one Au NP was according to Equation VIII.6.1 $\mathrm{m}_{\mathrm{NP}}=\mathrm{m}_{\mathrm{c}}+\mathrm{m}_{\mathrm{NP} \text {, org }}=8.0 \times 10^{-19} \mathrm{~g}+3.2 \times 10^{-19} \mathrm{~g} \approx$ $11.2 \times 10^{-19} \mathrm{~g}$. The organic coating thus contributed $\mathrm{m}_{\mathrm{NP}, \text { org }} / \mathrm{m}_{\mathrm{NP}}=3.2 \times 10^{-19} \mathrm{~g} / 11.2 \times 10^{-19}$ $g \approx 29 \%$ of the mass of one NP. The contribution of the organic coating for the dodecanethiol-capped Au NPs overcoated with PMA was thus higher than for the 
hydrophobic DDT-Au NPs, as expected. Note that for bigger NP cores the percentage contribution of the organic coating would be much lower, but in the case of very small NPs it is highly relevant.
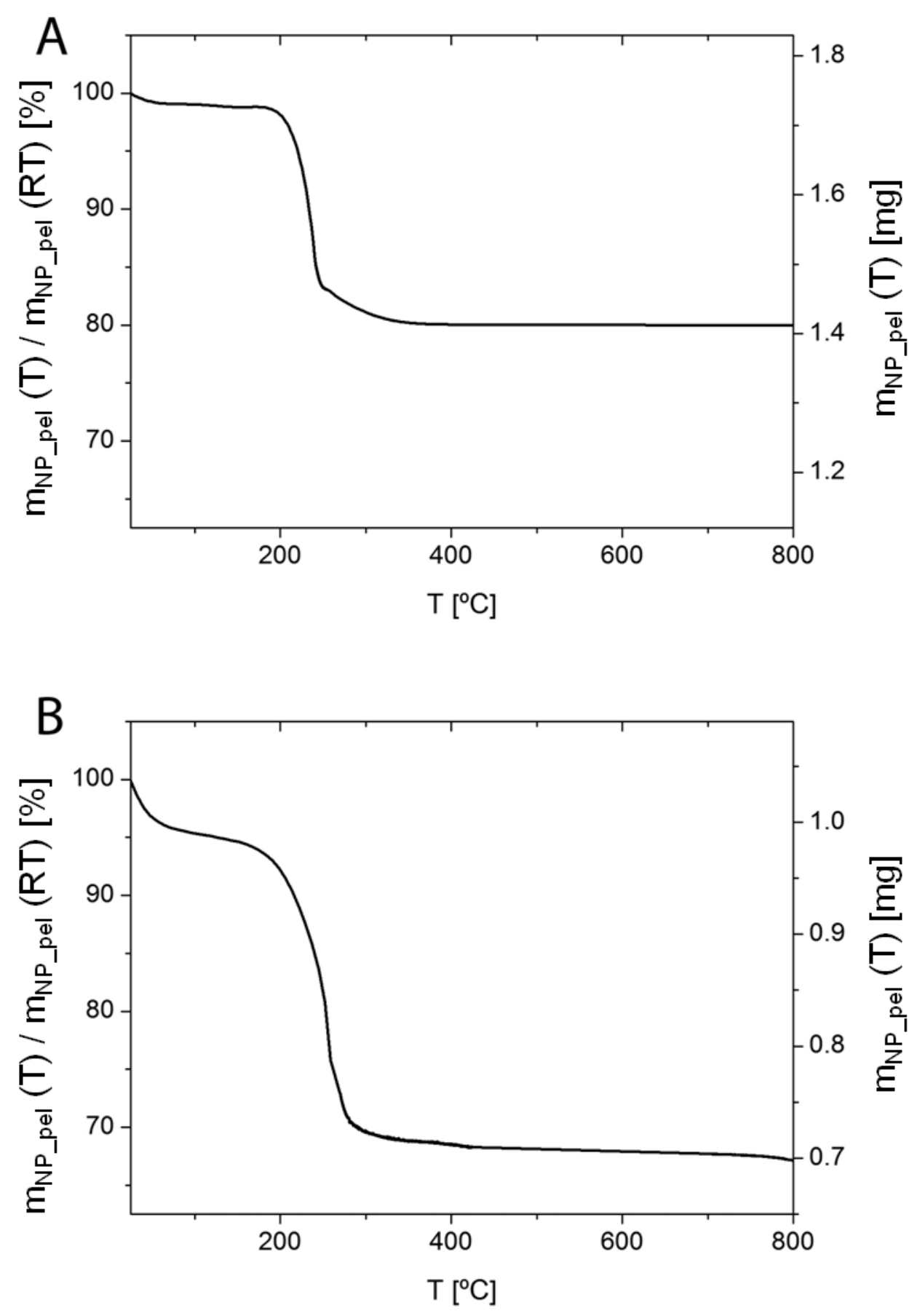

Figure VIII.6.1. TGA analyses of DDT-Au NPs (A) and DDT-Au NPs overcoated with PMA (B). In both graphs, $m_{N P \_p e l}(T)$ describes the mass of the NP pellet at temperature $T$. The masses are normalized to the mass $m_{N P_{-} p e l}(R T)$ at $R T$. The same scale in the left $y$-axes is used to facilitate the comparison. For both samples $d_{c}=4.3 \mathrm{~nm}$. 
The TGA analysis of dried PMA ( $c f . \S \mathrm{VI} .2$ ) is shown in Figure VIII.6.2. Note that PMA decomposed from 200 to ca. $500{ }^{\circ} \mathrm{C}$, but in the PMA-coated Au NPs (Figure VIII.6.1B) the main mass loss took place from 200 to $300{ }^{\circ} \mathrm{C}$, and the mass of the sample remained almost constant above this temperature. The mass loss observed in the PMA polymer above $\mathrm{ca} .350^{\circ} \mathrm{C}$ may thus have corresponded to the evaporation of impurities, which may have originated from the precursors used for its synthesis. By using ultracentrifugation ( $c f . \S$ VII.2), such impurities can be removed from the coated NPs.

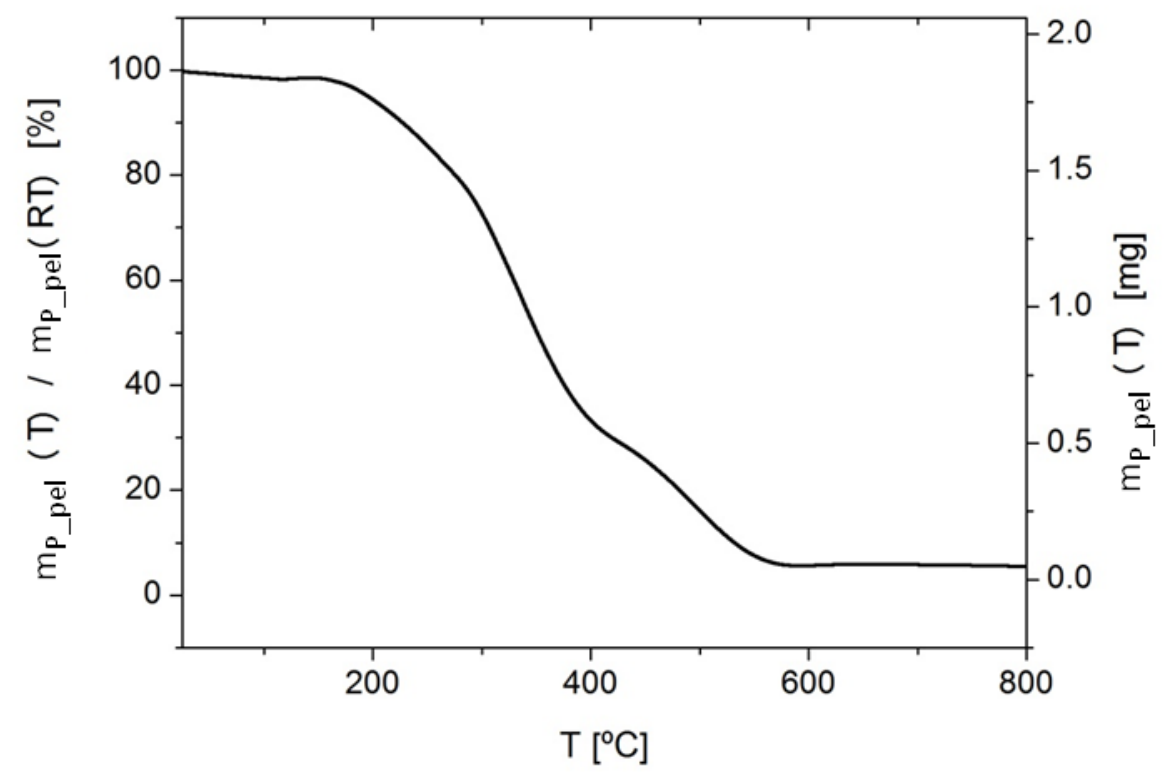

Figure VIII.6.2. TGA analysis of PMA. $m_{P_{-} \text {pel }}(T)$ describes the mass of the PMA at temperature $T$. The mass is normalized to the mass $m_{P_{-} p e l}(R T)$ at $R T$.

Based on these data some quantitative analysis concerning the organic surface coating can be performed. In the case of the DDT capped Au NPs the number $\mathrm{N}_{\mathrm{DDT} / \mathrm{NP}}$ of DDT molecules per Au NP can be calculated, by assuming that the organic surface coating only comprises DDT.

$$
m_{N P, o r g}=m_{N P, D D T}
$$

Using the molar mass of DDT $M_{\text {ligand }}=M_{D D T}=202.40$ Da thus leads to

$$
N_{D D T / N P}=\left(m_{N P, \text { org }} / M_{\text {ligand }}\right) \times N_{A}
$$

For the DDT-Au NPs $\left(d_{c}=4.3 \mathrm{~nm}\right)$ as analyzed here with TGA the following result was obtained as described above: $m_{N P, o r g}=1.7 \times 10^{-19}$ g. Thus $N_{D D T / N P}=\left(m_{N P, o r g} / M_{D D T}\right) \times N_{A}=$ 
$\left(1.7 \times 10^{-19} \mathrm{~g} / 202.40 \mathrm{~g} \cdot \mathrm{mol}^{-1}\right) \times 6.02 \cdot 10^{23} \mathrm{~mol}^{-1}=506$ DDT molecules are assumed to be situated at the surface of the DDT-Au NPs. The surface of one Au core is

$$
A_{c}=4 \pi \cdot\left(d_{c} / 2\right)^{2}=\pi \cdot d_{c}^{2}
$$

and thus in the present case $A_{c}=\pi \cdot(4.3 \mathrm{~nm})^{2} \approx 58.1 \mathrm{~nm}^{2}$. In this way the surface area which is occupied by each DDT molecule is

$$
A_{c, D D T}=A_{c} / N_{D D T / N P}
$$

(Equation VIII.6.5)

In the present case each DDT molecule covers $A_{c, D D T}=58.1 \mathrm{~nm}^{2} / 506 \approx 0.11 \mathrm{~nm}^{2}$ surface area. This is less than observed for the highest packing density of alkanethiols on regular smooth $\mathrm{Au}(111)$ surface of $0.214 \mathrm{~nm}^{2}{ }^{96}$. The discrepancy might be explained by several arguments. Due to the highly curved surface more thiols may be bound per surface area according to a radius-of-curvature effect, which leaves more space for the alkyl chains pointing towards solution ${ }^{52}$. Due to the typical size distribution of Au cores (such as shown in Figure VIII.3.1), there is a part of NPs with $d_{c}$ smaller than the average value and thus with a higher surface-to-volume ratio, and thus more thiols can bind. Despite purification there still may be excess ligand (DDT) in solution. The ligand exchange from TOAB-Au NPs to DDT-Au NPs may have been incomplete, leading to presence of ligands others than DDT on the Au surface. In addition the assumed monolayer formation of DDT may not be completely true and some more DDT may be attached to the NPs for example due to intercalation. TGA thus helps to verify assumptions about the structure of NPs.

Also for the PMA coated Au NPs some quantitative analysis can be performed ${ }^{97}$. In this case the mass of the organic surface coating around one NP comprises the underlying DDT layer $m_{N P, D D T}$ and the PMA-based polymer $m_{N P, P}$ wrapped around it.

$$
m_{N P, \text { org }}=m_{N P, D D T}+m_{N P, P}
$$

According to the TGA results in this section the DDT contributes to $17 \%$ of the mass of one DDT-Au NP, whereas the DDT plus PMA-based polymer contribute to $29 \%$ of the mass of one PMA-Au NP. Thus, based on the TGA data of $m_{N P, \text { org }}=3.2 \cdot 10^{-19} \mathrm{~g}$ for PMA$\mathrm{Au}$ NPs the following contributions can be calculated: $\mathrm{m}_{\mathrm{NP}, \mathrm{DDT}}=(17 \% / 29 \%) \times \mathrm{m}_{\mathrm{NP} \text {,org }}=$ $(17 / 29) \times 3.2 \cdot 10^{-19} \mathrm{~g} \approx 1.9 \cdot 10^{-19} \mathrm{~g}$, and $\mathrm{m}_{\mathrm{NP}, \mathrm{P}}=((29 \%-17 \%) / 29 \%) \times \mathrm{m}_{\mathrm{NP}, \text { org }}=(12 / 29) \mathrm{x}$ $3.2 \cdot 10^{-19} \mathrm{~g} \approx 1.3 \cdot 10^{-19} \mathrm{~g}$. By using the molar mass of one polymer monomer (cf. Equation VI.2.1), the number of polymer monomer bound per NP can be calculated as

$$
N_{P / N P}=\left(m_{N P, P} / M_{P}\right) x N_{A}
$$


Using the value of $\mathrm{M}_{\mathrm{P}}=293 \mathrm{~g} / \mathrm{mol}$ for PMA-based polymers as calculated in $\S \mathrm{VI} .2$ leads to $N_{P / N P}=\left(m_{N P, P} / M_{P}\right) \times N_{A}=\left(1.3 \cdot 10^{-19} \mathrm{~g} / 293 \mathrm{~g} / \mathrm{mol}\right) \times 6.02 \cdot 10^{23} \mathrm{~mol}^{-1} \approx 270$ polymer monomer units bound to each PMA-Au NP. This value can be now compared to the number of polymer monomer units $\mathrm{N}_{\mathrm{P} / \mathrm{NP} \text { (added) }}$ that have been added to each DDT-Au NP during the formation of PMA-Au NPs.

$$
\mathrm{N}_{\mathrm{P} / \mathrm{NP} \text { (added) }}=\mathrm{R}_{\mathrm{P} / \mathrm{Area}} \times \mathrm{A}_{\text {eff }}
$$

(Equation VIII.6.8)

In the present case the following parameters were used: $\left.\right|_{\text {ligand }}=I_{D D T}=1 \mathrm{~nm}$ (cf. Table VI.3.1), resulting in $d_{\text {eff }}=d_{c}+\left.2 x\right|_{\text {ligand }}=4.3 \mathrm{~nm}+2 \times 1 \mathrm{~nm}=6.3 \mathrm{~nm}$, and thus according to Equation VI.3.1 in $A_{\text {eff }}=\pi \cdot d_{\text {eff }}{ }^{2}=\pi \cdot(6.3 \mathrm{~nm})^{2} \approx 124 \mathrm{~nm}^{2}$. Therefore, $N_{P / N P(a d d e d) ~}=R_{P / A r e a ~} X$ $A_{\text {eff }}=100 \mathrm{~nm}^{-2} \times 124 \mathrm{~nm}^{2}=12,400$ polymer monomer units had been added per NP

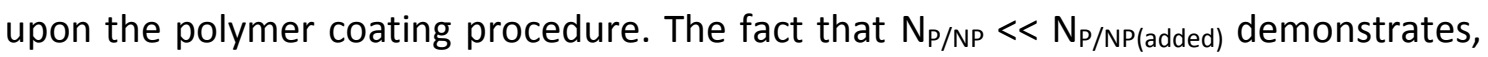
that most added polymer actually has not bound to the NPs, and thus purification of NP solutions from excess polymer is crucial.

\section{VIII.7 Infrared spectroscopy}

Fourier-transformed infrared spectroscopy (FTIR) allows for analyzing the chemical structure of the organic shell around NP cores. As a vibrational spectroscopy with a specific vibrational fingerprint for specific functional groups, FTIR can be used to qualitatively describe the organic shell around NPs and thus can be employed to follow up surface functionalization or ligand exchange. Moreover, deviations in typical vibrational features of adsorbing functional groups on the surface of NPs can be used to describe the type and orientation of organic ligand-core interaction. In the present work FTIR analysis of DDT-Au NPs ( $\approx 4 \mathrm{~nm}$ core diameter, $c f . \S$ §I.2) before and after overcoating with PMA ( $c f$. §VI.3), as well as for free PMA as control, were carried out and compared. Spectra were measured with a Thermo Nicolet 6700 FT-IR. Sample purity is essential and thus initial the DDT-Au NP sample was purified as described above ( $c f . \S I .2$ ) and PMA-coated Au NPs ( $c f . \S \mathrm{VI} .3$ ) were purified following the protocol mentioned above ( $c f$. §VII.5), while PMA was used directly as-synthesized ( $c f$. §VI.2). DDT-Au NPs and PMA solutions were dissolved in chloroform, and they were left to evaporate to get solid samples, whereas the aqueous solution of PMA-coated Au NPs was lyophilized prior to the preparation of the $\mathrm{KBr}$ pellets. These pellets were prepared at a $1 \%$ concentration of the sample, i.e. $\mathrm{m}_{\mathrm{NP} \_ \text {pel }}=2 \mathrm{mg}$ of the dried NP sample was mixed with $200 \mathrm{mg}$ of $\mathrm{KBr}$, grinding until a homogeneous mixture was obtained, and finally the pellet was formed by using a hand press. For the measurements, 50 scans 
were recorded with a Thermo Nicolet $6700 \mathrm{FT}-\mathrm{IR}$, and the presented FTIR spectra of 50 scans at $4 \mathrm{~cm}^{-1}$ resolution were averaged. Data are presented in Figure VIII.7.1.

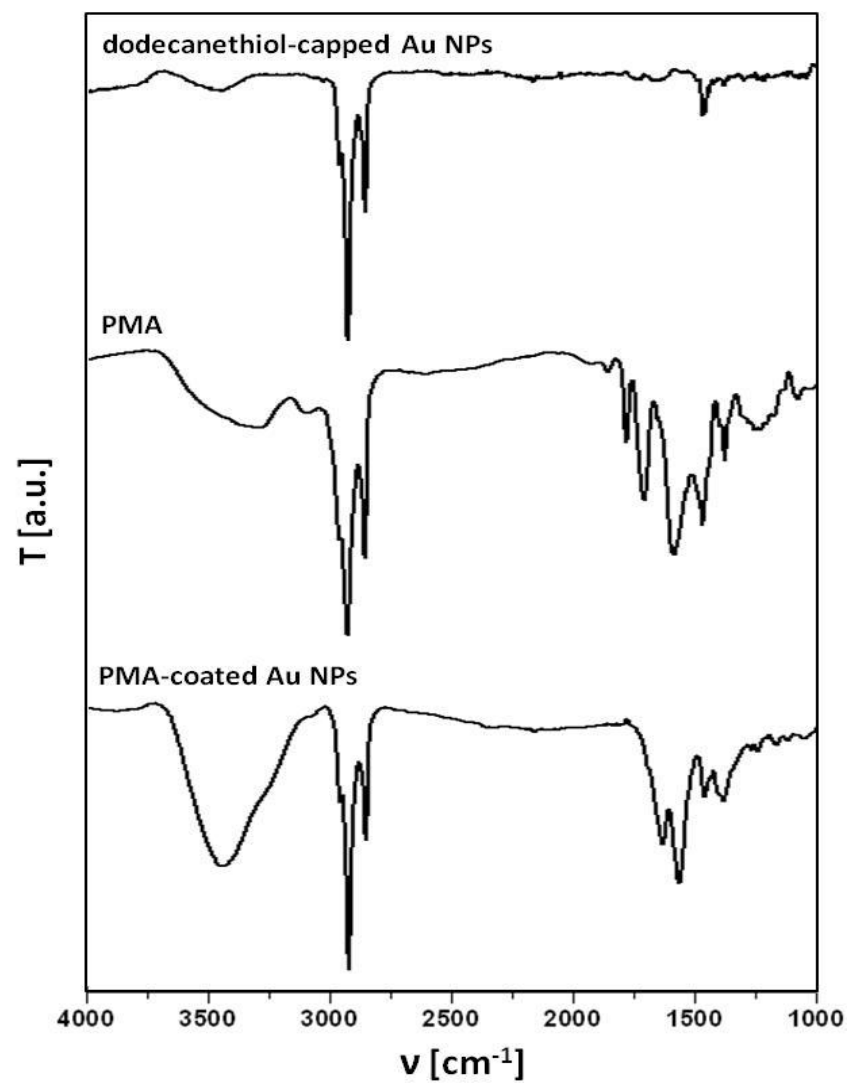

Figure VIII.7.1. FTIR spectrum (transmission mode $T(v)$, i.e. transmission $T$ versus wavenumber $v$ ) of DDT-Au NPs of $\approx 4 \mathrm{~nm}$ core diameter before and after overcoating with PMA. The spectrum of free PMA is shown as control.

The DDT-Au NPs exhibited only three significant peaks. The strong peaks at $2923 \mathrm{~cm}^{-1}$ and $2853 \mathrm{~cm}^{-1}$ were assigned to the asymmetric and symmetric $\mathrm{C}-\mathrm{H}$ stretching of the alkyl chain, respectively, and the peak at $1460 \mathrm{~cm}^{-1}$ was assigned to the bending vibrations of $\mathrm{C}-\mathrm{H}$. In contrast, the PMA polymer ( $c f$. Figure VI.2.1) presented more peaks. The strong peaks at $2925 \mathrm{~cm}^{-1}$ and $2854 \mathrm{~cm}^{-1}$ were assigned to the asymmetric and symmetric $\mathrm{C}-\mathrm{H}$ stretching of the alkyl chains. The broad and weak adsorption peak from $3200-3600 \mathrm{~cm}^{-1}$ corresponded to the presence of the $\mathrm{O}-\mathrm{H}$ stretching resonance of the carboxylic acids formed after the anhydride opening. The two peaks at $1780 \mathrm{~cm}^{-}$ ${ }^{1}$ and $1705 \mathrm{~cm}^{-1}$ were characteristic of the cyclic anhydride with 5-membered rings. The peak of the symmetric stretching vibrations of $\mathrm{C}=\mathrm{O}$ from the carboxylic groups overlapped with the peak at $1705 \mathrm{~cm}^{-1}$. At $1583 \mathrm{~cm}^{-1}$ the symmetric stretching vibrations of $\mathrm{N}-\mathrm{C}=\mathrm{O}$ of the amide groups appeared. The 1468 and $1375 \mathrm{~cm}^{-1}$ peaks were assigned to the angular deformation of $\mathrm{CH}_{2}$ and $\mathrm{CH}_{3}$, respectively. When the DDT-Au NPs were overcoated with the amphiphilic PMA polymer, the IR spectrum of 
the resulting PMA-coated Au NPs had the same peaks as the DDT-Au NPs and some additional peaks from the PMA, such as the peaks at 1635 and $1565 \mathrm{~cm}^{-1}$ assigned to the symmetric stretching vibrations of $\mathrm{C}=\mathrm{O}$ from the carboxylic groups and $\mathrm{N}-\mathrm{C}=\mathrm{O}$ from the amide groups, respectively. Here, again the 1466 and $1384 \mathrm{~cm}^{-1}$ peaks from the angular deformation of $\mathrm{CH}_{2}$ and $\mathrm{CH}_{3}$ appeared, respectively. Additionally, there was a broad and intense band from $3000-3600 \mathrm{~cm}^{-1}$ corresponding the $\mathrm{O}-\mathrm{H}$ stretching resonance of the carboxylic acids formed when the NPs had been transferred to water and then all the anhydride rings had been opened. Collectively, FTIR results indicated the surface modification (overcoating) of DDT-Au NPs with PMA.

In summary, FTIR analysis of NPs allows for knowing which ligands and chemical groups are present on the NP surface. This serves to confirm that the functionalization strategy (e.g. polymer coating or ligand exchange/displacement) worked properly.

\section{VIII.8 Nuclear Magnetic Resonance}

Nuclear magnetic resonance spectroscopy (NMR) is a powerful tool to probe the chemical structure of the organic shell around the inorganic NP core. NMR is mainly used to provide evidence on the presence and orientation of organic molecules/ligands around NPs. NMR works well to analyze small molecules, but it may get complicated in the case of large constructs, due to the heterogeneity and reduced flexibility of the analyzed constructs.

In the present work we analyzed the NMR spectra of PMA before and after the coating of $\approx 4 \mathrm{~nm}$ DDT-Au NPs (cf. §l.2 and §VI.3). A ${ }^{1} \mathrm{H}-\mathrm{NMR}$ spectrum of PMA in $\mathrm{CDCl}_{3}$ (i.e. chloroform where the proton ${ }^{1} H$ was substituted by a deuteron $D={ }^{2} H$ ) was first measured. As expected non-resolved broad bands were observed as a result of the size distribution of the starting poly(isobutylene-alt-maleic anhydride)-graft-dodecyl and the random distribution of dodecylamine moieties along the polymer chain. In this situation it was possible to distinguish broad singlets ( $\mathrm{br} s$ ) between 3.66 and $2.63 \mathrm{ppm}$ corresponding to $-\mathrm{CH}$ - protons, $1.69-0.92 \mathrm{ppm}$ corresponding to $-\mathrm{CH}_{2}-$ protons, and 0.90-0.80 ppm corresponding to $-\mathrm{CH}_{3}$ - protons (cf. Figure VIII.8.1A). A ${ }^{13} \mathrm{C}-\mathrm{NMR}$ spectrum was also measured observing good resolution for non-quaternary carbon atoms due to their higher signal intensity compared to quaternary ones such as $C=0$, for instance ( $c f$. Figure VIII.8.1B). The peak assignment both, in ${ }^{1} \mathrm{H}$ and ${ }^{13} \mathrm{C}-\mathrm{NMR}$, was based on the chemical shift $(\delta)$ and was aided by 2D HSQC experiment (Heteronuclear Single Quantum Correlation) edited for distinguishing $\mathrm{CH}_{2}$ signals from $\mathrm{CH}$ and $\mathrm{CH}_{3}$. 


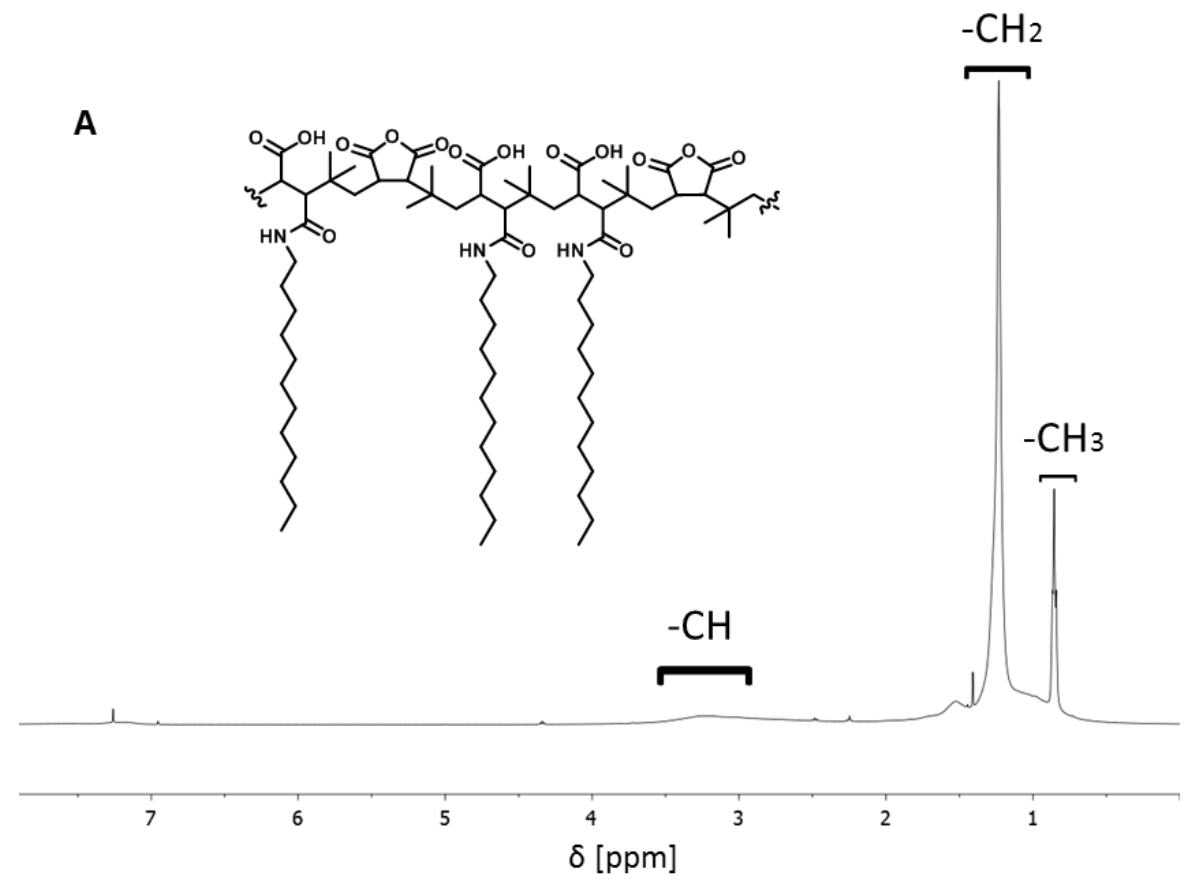

B

$\underline{\mathrm{C}}-\left(\mathrm{CH}_{3}\right)_{2}$

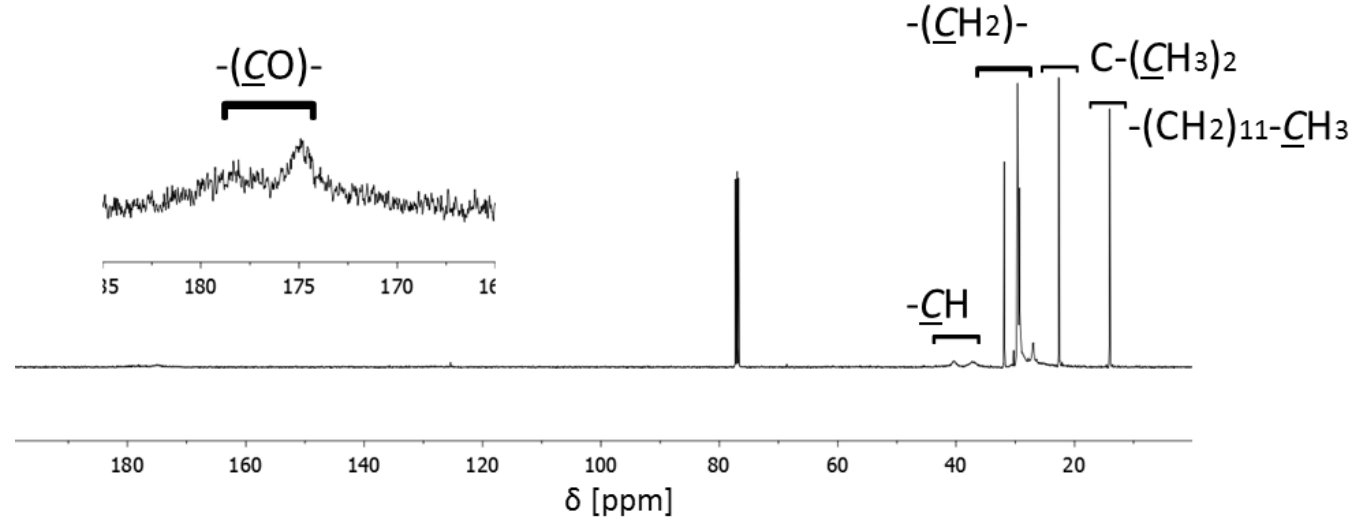

Figure VIII.8.1. A) ${ }^{1} \mathrm{H}-\mathrm{NMR}$ spectrum of PMA in $\mathrm{CDCl}_{3}$. B) ${ }^{13} \mathrm{C}-\mathrm{NMR}$ spectrum of PMA in $\mathrm{CDCl}_{3}$, the zoomed area shows the peaks for the carbonyl groups.

${ }^{1} \mathrm{H}$-NMR of PMA coated Au NPs ( $\approx 4 \mathrm{~nm}$ core diameter, $c f . \S$ §. 2 ) was measured in water with $15 \%$ of deuterium oxide, using a sequence for suppressing water signal. When PMA was coating the Au NPs, there were no longer cyclic anhydrides present and two separate peaks were observed for $-\mathrm{CH}$ protons: those linked to amide groups and kept inside the hydrophobic core, and those linked to carboxylic groups and exposed to the water phase. Those nuclei had very different environments and hence different chemical shifts. Interestingly, when this spectrum was compared with that of PMA treated with $\mathrm{NaOH}$ to open all the anhydride groups and measured under the same conditions $\left(\mathrm{H}_{2} \mathrm{O}\right.$ with $\left.15 \% \mathrm{D}_{2} \mathrm{O}\right)$ a somehow different profile was observed. The $\mathrm{CH}$ peak was barely visible and only the $\mathrm{CH}_{2}$ and $\mathrm{CH}_{3}$ peaks were clearly visible, although the ratio between them was modified $\left(\mathrm{CH}_{2} / \mathrm{CH}_{3} \approx 2\right.$ in PMA coated Au NPs vs. $\mathrm{CH}_{2} / \mathrm{CH}_{3}$ 
$\approx 1$ in PMA after $\mathrm{NaOH}$ treatment). This shows that PMA molecules arranged differently when free in solution (i.e. forming empty polymer micelles) or when on the surface of Au NPs, but it is difficult to extract further structural information from these experiments.
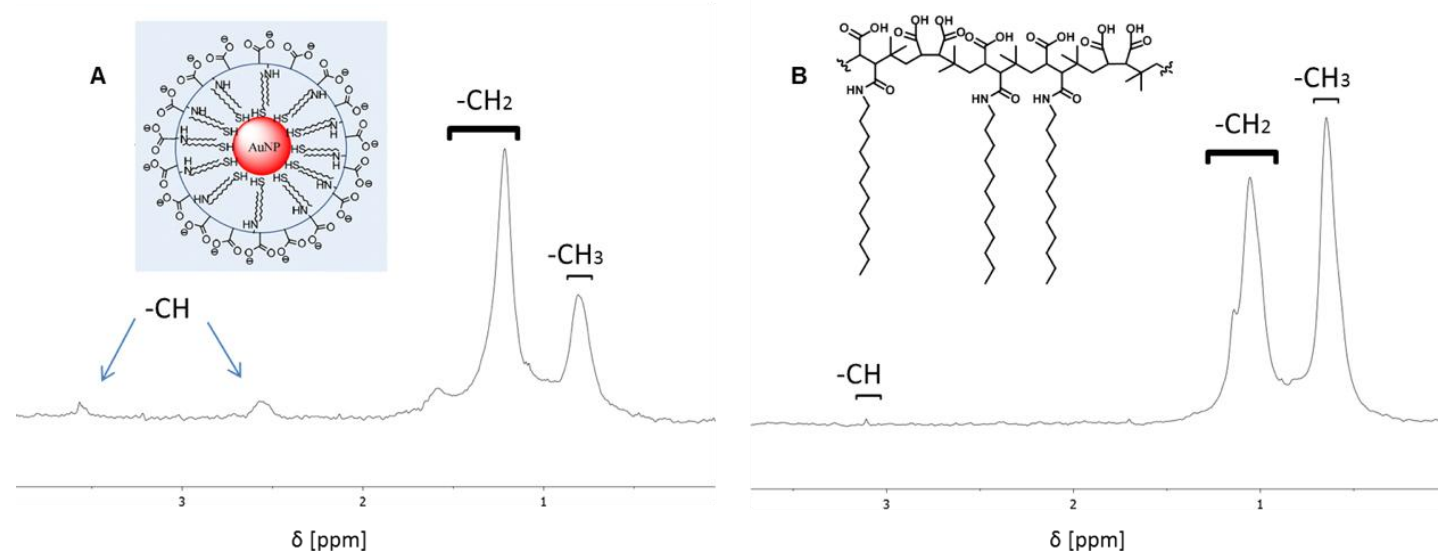

Figure VIII.8.2. A) ${ }^{1} \mathrm{H}-\mathrm{NMR}$ spectrum of PMA coated Au NPs of $\approx 4 \mathrm{~nm}$ core diameter in $15 \% \mathrm{D}_{2} \mathrm{O} / \mathrm{H}_{2} \mathrm{O}$ mixture. B) ${ }^{1} \mathrm{H}-\mathrm{NMR}$ of PMA in $15 \% \mathrm{D}_{2} \mathrm{O} / \mathrm{H}_{2} \mathrm{O}$ mixture after treatment with base to open all anhydride groups.

In summary, NMR spectroscopy can give further evidence of the presence of PMA on the surface of Au NPs of $\approx 4 \mathrm{~nm}$ and can also be used to check the purity of those NPs by confirming the absence of other organic molecules such as organic solvents or glycerol from the filters. However, the heterogeneity of PMA polymer and the poor ability of some of the protons to resonate when linked to Au NPs led to poor resolution spectra that could only be interpreted from a qualitative point of view.

${ }^{1} \mathrm{H}$ and ${ }^{13} \mathrm{C}$ spectra were recorded in a Bruker $500 \mathrm{MHz}$ spectrometer. Chemical shifts are reported in parts per million (ppm) relative to the residual signal of the solvent used, and are listed below:

PMA (cf. Figure VIII.8.1). ${ }^{1} \mathrm{H}$ NMR (500 MHz, $\left.\mathrm{CDCl}_{3}\right) \delta 3.66-2.63$ (br s, CH), $1.69-0.92$ $\left(\mathrm{m}, \mathrm{CH}_{2}\right), 0.90-0.80\left(\mathrm{~m}, \mathrm{CH}_{3}\right) .{ }^{13} \mathrm{C} \mathrm{NMR}\left(126 \mathrm{MHz}, \mathrm{CDCl}_{3}\right) \delta 178.08,174.80,173.92$, 77.00, 40.36, 37.19, 31.86, 30.25, 29.59, 29.30, 29.14, 28.78, 26.98, 22.62, 14.05.

PMA coated Au NPs (cf. Figure VIII.8.2A). ${ }^{1} \mathrm{H}$ NMR (500 MHz, $\left.\mathrm{D}_{2} \mathrm{O}\right) \delta 3.66(\mathrm{br} \mathrm{s}, \mathrm{CH})$, 2.64 (br s, CH), 1.68 (br s, $\mathrm{CH}_{2}$ ), 1.31 (br s, $\mathrm{CH}_{2}$ ), 0.89 (br s, $\mathrm{CH}_{3}$ ).

PMA after basic treatment (cf. Figure VIII.8.2B). ${ }^{1} \mathrm{H}$ NMR $\left(500 \mathrm{MHz}, \mathrm{D}_{2} \mathrm{O}\right) \delta 2.40$ (br s, $\mathrm{CH}), 1.08$ (br s, $\mathrm{CH}_{2}$ ), 0.67 (br s, $\mathrm{CH}_{3}$ ). 


\section{Characterization of NPs' photophysical properties}

\section{IX.1 UV/vis absorption spectroscopy \\ IX.2 Determination of NP concentrations from absorption spectra \\ IX.3 Fluorescence spectroscopy}

IX.4 Determination of quantum yields

\section{IX.1 UV/vis absorption spectroscopy}

UV/vis absorption spectroscopy is an easy and simple method to characterize important properties of colloidal NPs. Several types of NPs, such as quantum dots or plasmonic NPs have particular features (i.e. resonance peaks) in their absorption spectra, which provide important information regarding NP size, size-distribution, and shape. Moreover, UV/vis absorption spectroscopy can be used to determine the concentration of NPs (cf. §IX.2) and to follow colloidal growth and stability (cf. $\S$ X.1).

To record absorption spectra for NPs, several issues need to be considered. First, a blank has to be recorded for the pure solvent, which needs to be subtracted from the NP absorption spectrum. Typically (unless for example IR absorbing NPs such as gold nanorods are investigated) NP suspensions are transparent at high wavelengths. Spherical Au NPs of a few $\mathrm{nm}$ in size have their plasmon absorption peak around 520 $\mathrm{nm}$ (cf. Table IX.1.1) and do not absorb light at higher wavelengths of $c a .800-1200$ $\mathrm{nm}$. In case the measured "absorption" in this range does not converge to zero, in fact scattering and not absorption has been detected. This most likely is due to partial agglomeration of the NP sample ( $c f . \S X .1$ ). Thus, before further analysis based of the UV/vis absorption spectra of a NP solution, it has to be made sure that the absorption in the spectra converges towards zero in this range (ca. $800-1200 \mathrm{~nm}$ ). In case the NPs are well dispersed this should be automatically the case after automated subtraction of the offset. However, in case the offset function does not work, a constant offset may be subtracted manually, until the absorption for high wavelengths (ca. $800-1200 \mathrm{~nm}$, in case there are no absorption features of the NPs in this range) converges to zero, see Figure IX.1.1. 

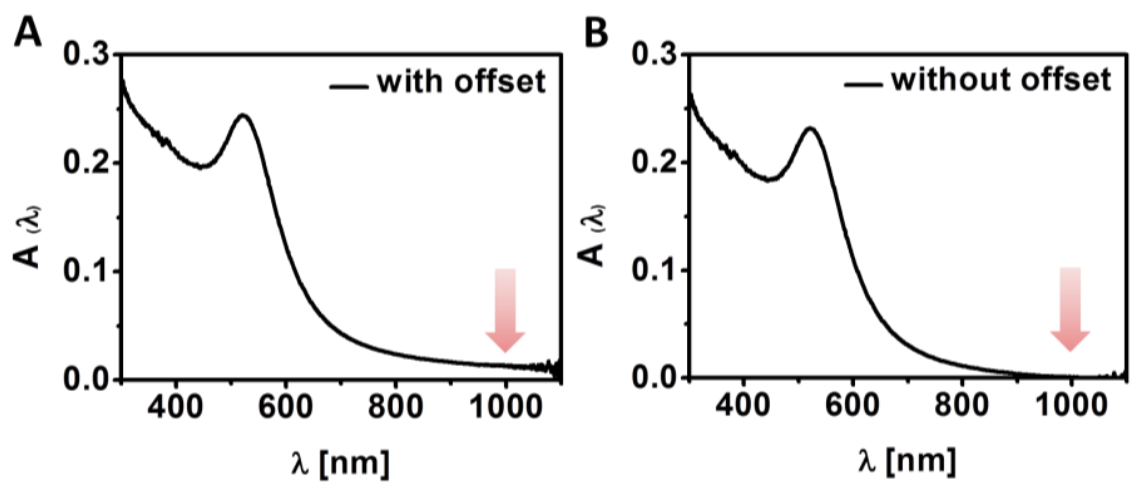

Figure IX.1.1. Absorption spectra (absorption $(A)$ as function of wavelength $\lambda$ ) of $\approx 4$ $n$ m core diameter Au NPs (cf. §I.2) coated with PMA in water (cf. §VI.3). A) The "raw spectra" includes an offset at higher wavelengths. B) Processed spectra by subtracting the absorption at higher wavelengths from the whole spectra. Processed spectra provide more accurate comparison between different spectra with different offset levels.

Next, it is important to use a cuvette of an appropriate material. Plastic cuvettes may be incompatible with organic solvents (e.g. acetone and chloroform), which should be avoided to eliminate artifacts in recording absorption spectra of NPs. Moreover, cuvettes are not transparent in the whole UV/vis absorption spectrum. In case that the absorbance at small wavelengths $(<300 \mathrm{~nm})$ is important, the material of the used cuvette plays an important role. Plastic cuvettes are not transparent at short wavelengths $(<250 \mathrm{~nm})$, and thus should be avoided in these cases. Even glass cuvettes show significant absorption at short wavelengths. The use of quartz cuvettes is standard practice to minimize cuvette-related absorption at short wavelengths (see Figure IX.1.2).

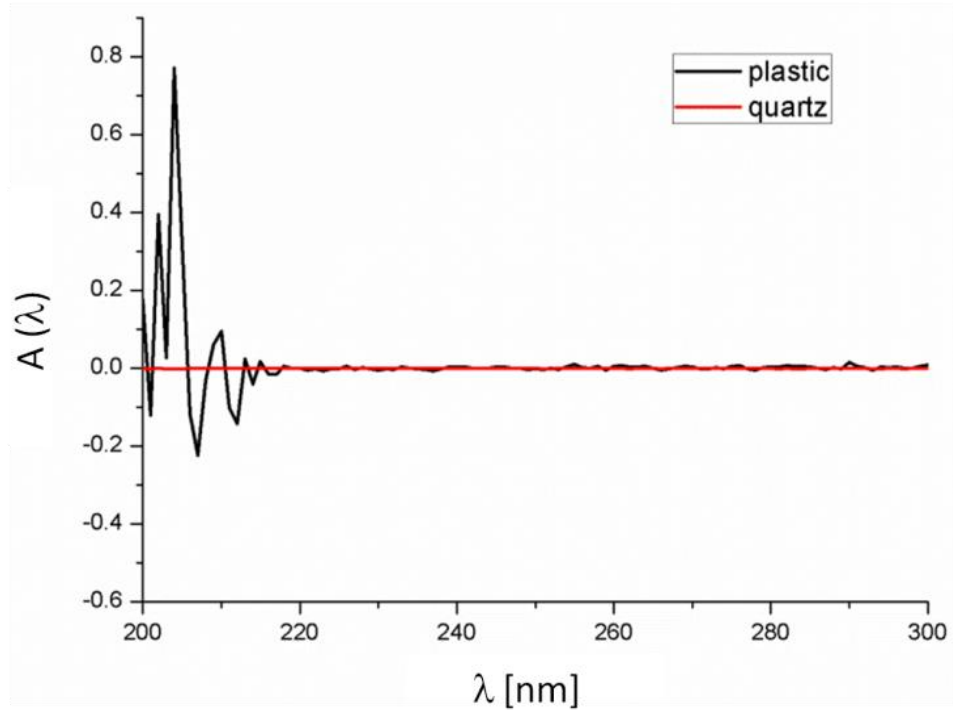


Figure IX.1.2. Absorption spectrum $A(\lambda)$ of water (with water as blank) recorded in plastic or quartz cuvette. Note the cuvette-related absorption when plastic cuvette was used at short wavelength.

Proper sample dilution is essential to record accurate UV/vis spectra of NP solutions. If the sample is very concentrated, the recorded spectra will be noisy, due to saturation of instruments detector. Moreover, quantification will not be possible since absorbance readings will be out of the linear range of concentration-absorbance relationship.

The absorption $A(\lambda)$ is the logarithmic ratio of the intensity of the incident light beam $I_{0}$ to the intensity of the transmitted light beam $I_{t}(\lambda)$. For this reason the absorption also has no units. In case the path length of the cuvette is $I_{\text {sol }}=1 \mathrm{~cm}$, the absorption is also referred to as optical density (OD) of the solution.

$$
A(\lambda)=\log \left(I_{0} / I_{t}(\lambda)\right)
$$

In case the transmitted light is ten times less, due to the decadic logarithm, the absorption is a factor of 2 higher. Thus, for high absorptions rates the intensity of the transmitted light becomes very low and cannot be longer correctly detected.

Figure IX.1.3 shows the UV/vis absorption spectra of Au NPs as function of sequential dilution, as recorded on an Agilent $8450 \mathrm{UV} / \mathrm{vis}$ spectrometer. At high NP concentrations, the absorption spectrum is noisy and distorted at lower wavelengths, where the absorption is high and exceeding the capacity of the detector. Thus, absorption spectra need to be recorded at sufficiently low concentrations by accurate dilution with solvent. Typical optical densities should not exceed 0.5 and at higher absorption the shape of the spectrum may be an artifact. 


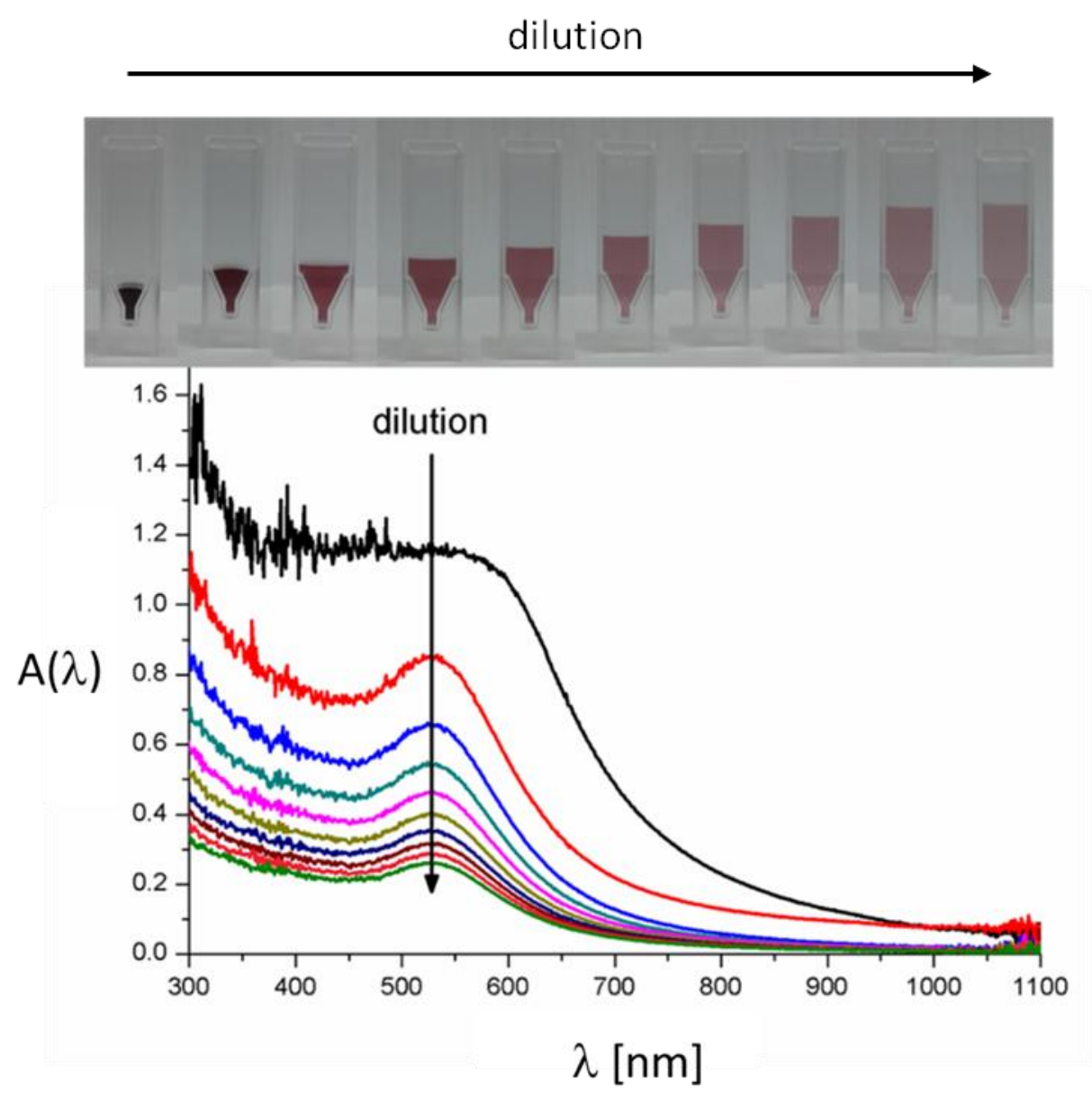

Figure IX.1.3. A concentrated aqueous solution of Au NPs was stepwise diluted, which can be seen by the fading color in solution (upper panel) and deceasing absorption intensity (lower panel). High NP concentration (black curve) resulted in high absorption, and thus $\lambda_{\max }$ cannot even be identified due to detector saturation that results in peak broadening and distortion.

Despite the strong plasmon optical extinction of Ag NPs and Au NPs, NPs from other materials may have no features in their UV/vis absorption spectra. Figure IX.1.4 shows as examples of such cases the absorption spectra for $\approx 4 \mathrm{~nm}$ FePt (cf. §l.4), $\approx 4 \mathrm{~nm}$ $\mathrm{Fe}_{3} \mathrm{O}_{4}$ (cf. §।.5), and $\approx 11 \mathrm{~nm} \mathrm{Sn} \mathrm{(cf.} \mathrm{§I.6)} \mathrm{NPs} \mathrm{dispersed} \mathrm{in} \mathrm{organic} \mathrm{solvent.} \mathrm{These} \mathrm{NP}$ solutions have higher absorption in the UV region (with no peaks), which converges to zero in the IR. 


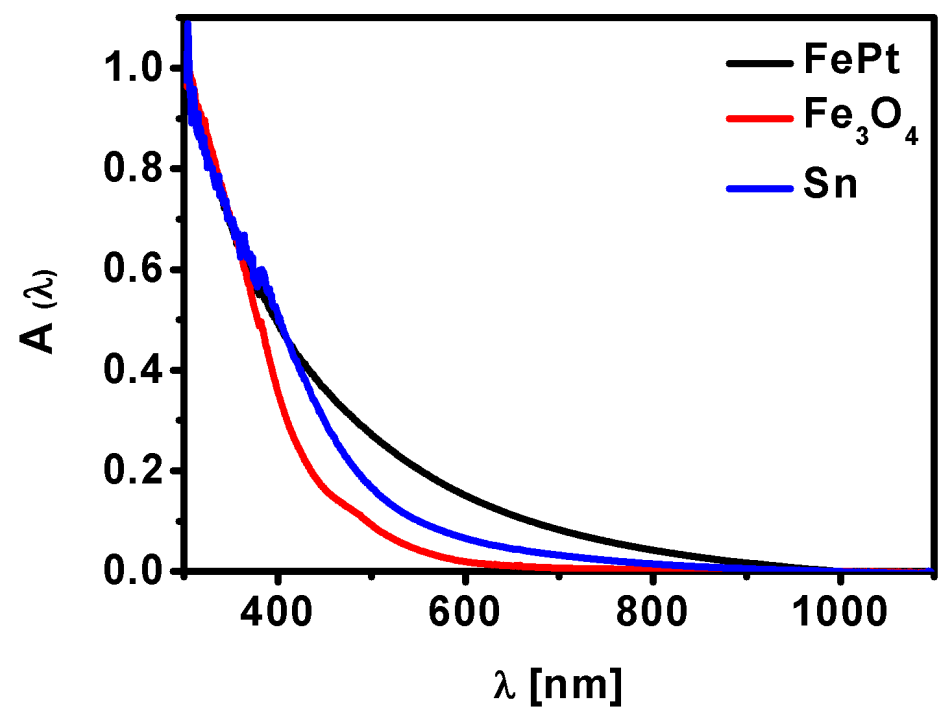

Figure IX.1.4. Normalized absorption spectra $A(\lambda)$ of FePt NPs (black curve) (cf. §I.4), $\mathrm{Fe}_{3} \mathrm{O}_{4} \mathrm{NPs}$ (red curve) (cf. §l.5), and Sn NPs (blue curve) (cf. §l.6). All NPs are dispersed in chloroform. Due to the absence of plasmon resonance, the absorption spectra do not contain defined peak or characteristics.

Plasmonic NPs (such as Au NPs, Ag NPs) have characteristic absorption peaks due to the surface resonance plasmon (SPR) phenomenon in the UV/vis-NIR region. Plasmonic NPs may have one or even more SPR peaks, depending on the shape and architecture of the NPs ${ }^{98-100}$. The characteristic absorption peaks are responsible for the distinctive bright color of NP solutions. The spectral position and shape of the surface plasmon absorption peak depends on a variety of factors, such as NP material, size, sizedistribution, shape, surface chemistry, state of aggregation, as well as of the solvent. Figure IX.1.5A shows a red shift of the SPR maximum ( $\left.\lambda_{\text {SPR }}\right)$ to higher wavelength when the sized of Au NPs is increased from $13 \mathrm{~nm}$ to $20 \mathrm{~nm}$ (size dependency). Figure IX.1.5B shows the absorption spectra of citrate capped spherical Ag NPs in the violet/UV ( $\lambda_{\text {SPR }}$ $=415 \mathrm{~nm}$ ) as compared to Au NPs with the same size (15 and $13 \mathrm{~nm}$ respectively) that absorb green light $\left(\lambda_{\text {SPR }}=520 \mathrm{~nm}\right)$ (NP material dependency). 

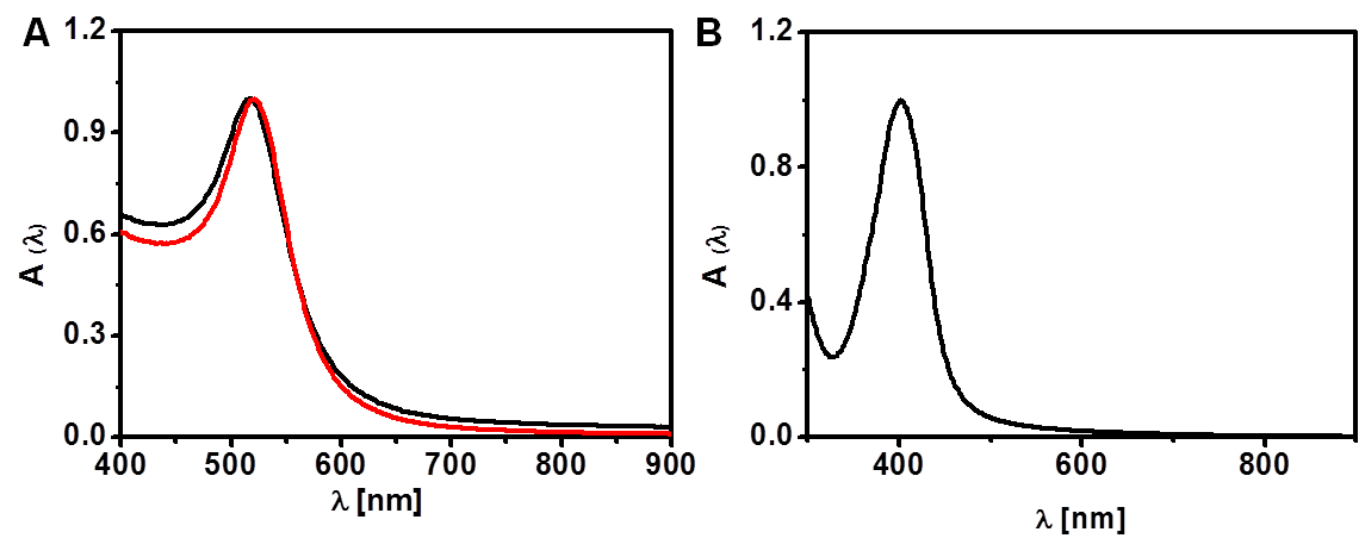

Figure IX.1.5. Normalized UV/vis absorption spectra of A) Cit-Au NPs $\approx 13 \mathrm{~nm}$ core diameter (black line) versus Cit-Au NPs $\approx 20 \mathrm{~nm}$ core diameter (red line). B) Cit-Ag NPs $\approx 15 \mathrm{~nm}$ core diameter. Au NPs and Ag NPs suspensions in water were prepared as detailed in \$II.2 and \$II.3, respectively.

The size-dependency of $\lambda_{\text {SPR }}$ for Au NPs is well documented with a general trend predicting a red shift of the absorption $\left(\lambda_{\text {SPR }}\right)$ as the NP diameter increases. Figure IX.1.6 shows a red shift of the SPR maximum ( $\left.\lambda_{\text {SPR }}\right)$ to higher wavelength, when Cit-Au NPs increase in size from $5 \mathrm{~nm}$ to $100 \mathrm{~nm}$. In case of Cit-Au NPs (green curve in B), the size distribution of the NPs is poor, i.e. there are NPs of different sizes in solution, and the absorption peak becomes broader. Thus, a sharp SPR peak is a good indication of narrow size distribution of the core diameter $d_{c}$. Figure IX.1.7 and Table IX.1.1 show a red shift of the SPR maximum wavelength $\left(\lambda_{\mathrm{SPR}}\right)$ to higher wavelengths, when commercially available Cit-Au NPs increased in size from $5 \mathrm{~nm}$ to $50 \mathrm{~nm}$.
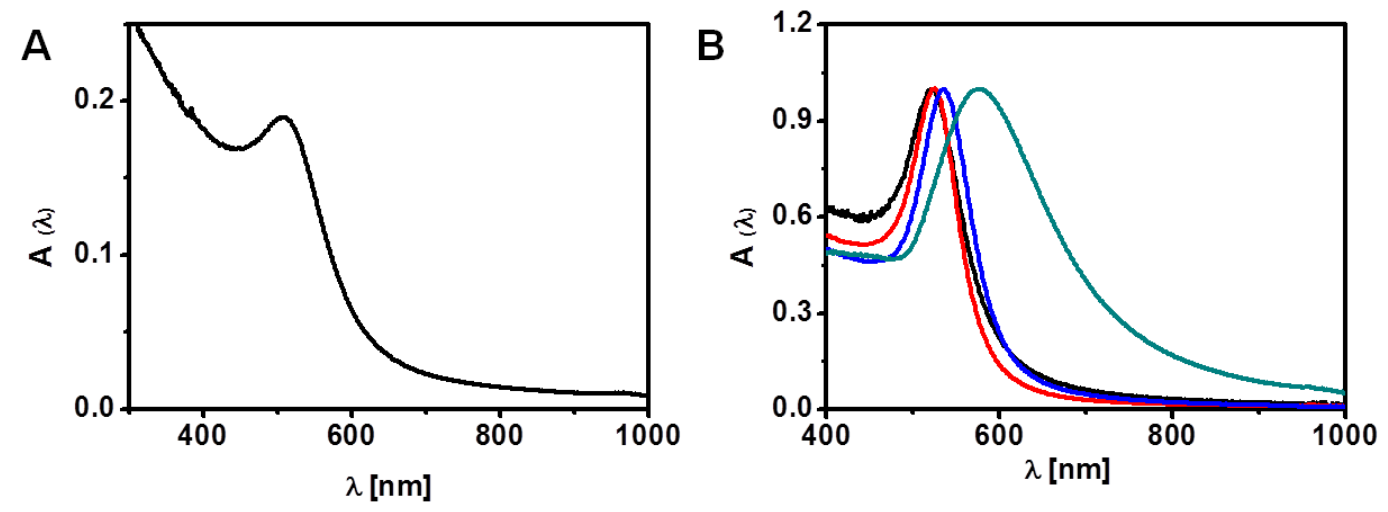

Figure IX.1.6. Normalized UV/vis absorption spectra of Cit-Au NPs (cf. §ll.2) with core diameter of $\approx 5 \mathrm{~nm}(A), 18 \mathrm{~nm}$ ( $B$, black line), $25 \mathrm{~nm}$ (B, red line), $50 \mathrm{~nm}$ (B, blue line), 
and $100 \mathrm{~nm}(B$, green line). The corresponding TEM images of these NPs are shown in VIII.3.7 A, D, E, F, G.

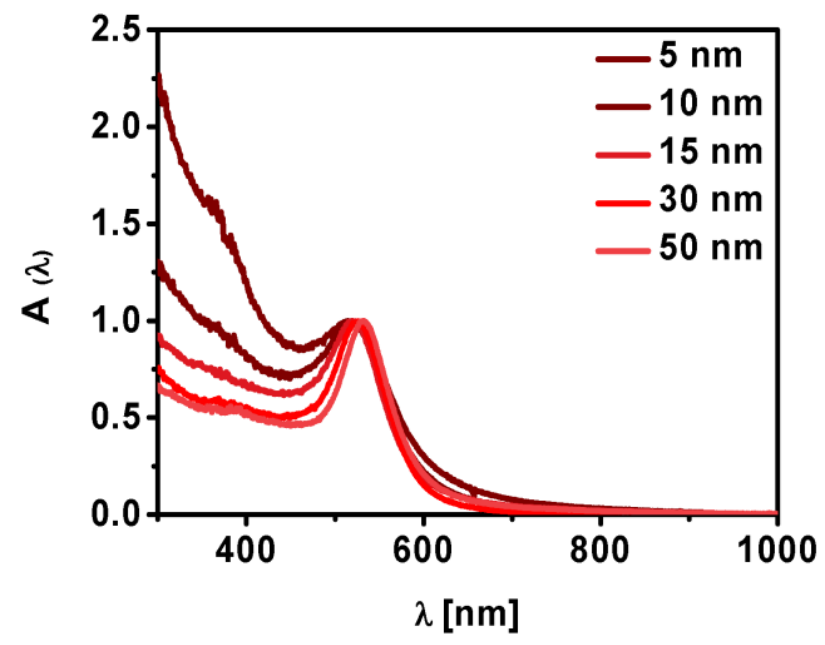

Figure IX.1.7. Normalized UV/vis absorption spectra of commercial Cit-Au NPs in water from BBI solutions (cf. §ll.2) with core diameter of 5, 10, 15, 30, and 50.

\begin{tabular}{|c|c|c|c|}
\hline $\mathrm{d}_{\mathrm{c}}[\mathrm{nm}]$ & Source & $\lambda_{\text {SPR }}[\mathrm{nm}]$ & $\varepsilon_{\mathrm{NPP}}\left[\mathrm{M}^{-1} \cdot \mathrm{cm}^{-1}\right]$ \\
\hline 4.2 & $\S$ I.2, §VI.3 & 516 & $8.70 \times 10^{6}$ \\
\hline 5 & BBI solutions & 518 & $9.70 \times 10^{6}$ \\
\hline 10 & BBI solutions & 520 & $9.55 \times 10^{7}$ \\
\hline 15 & BBI solutions & 523 & $3.64 \times 10^{8}$ \\
\hline 30 & BBI solutions & 526 & $3.58 \times 10^{9}$ \\
\hline 50 & BBI solutions & 532 & $1.93 \times 10^{10}$ \\
\hline
\end{tabular}

Table IX.1.1. Values of surface plasmon resonance maximum wavelength $\left(\lambda_{\max }\right)$, and molecular extinction coefficients, $\varepsilon_{N P},(c f . \S I X .2)$ of Cit-Au NPs of different size in water. Extinction coefficients were provided from the manufacturer.

The shape dependence of the SPR peak is well documented and reported in the literature. For example, rod-shaped Au NPs have two SPR peaks, as the oscillation of electrons in the conduction band can occur either around the short axis or the long axis, resulting in the so called transverse and longitudinal peaks, respectively. While in case of $\mathrm{Au}$ NPs the transverse plasmon peak is located generally in the visible region ( $520 \mathrm{~nm}$ ), the longitudinal peak can be tuned to be in the visible-Near infrared (Vis$\mathrm{NIR}$ ) region of the spectrum (600-1200 nm) as shown in Figure IX.1.8A. Spectra for starshaped Au NPs exhibit one peak in the Vis-NIR as shown in Figure IX.1.8B.

Finally, the surface plasmon peak can change dramatically upon agglomeration due to 
the plasmon coupling of aggregated NPs ${ }^{101}$. This will be discussed in more detail in $\S X .1$.
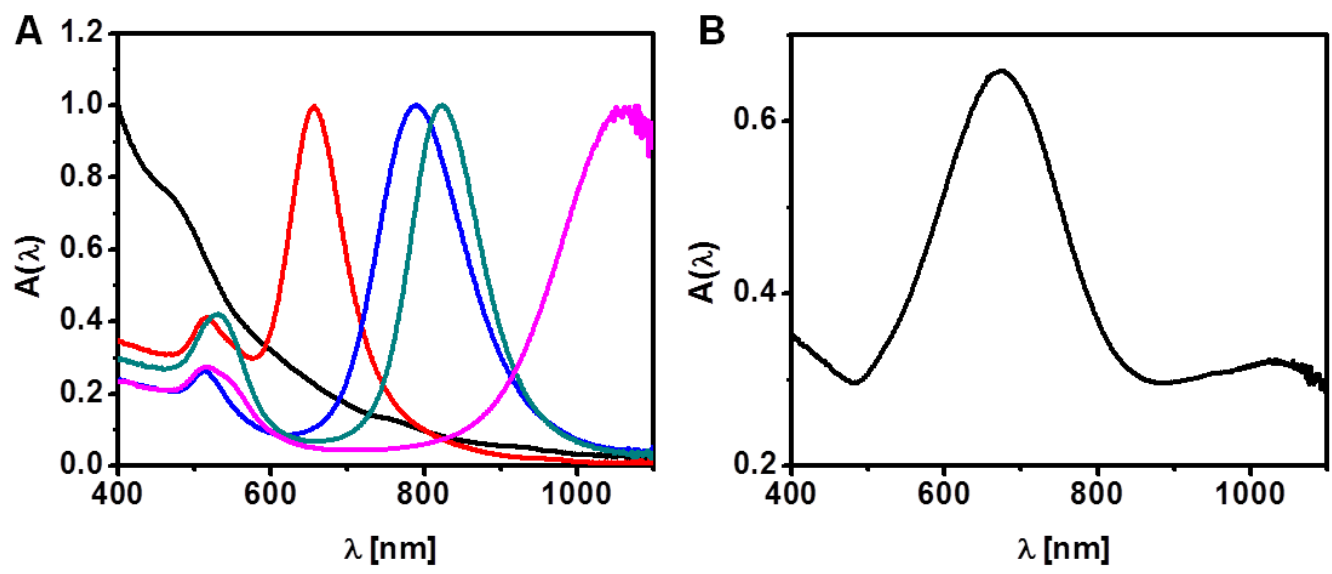

Figure IX.1.8. Normalized UV/vis absorption spectra of: A) Au NRs (cf. §II.4), with $\lambda_{S P R}=$ $650 \mathrm{~nm}$ (red curve), $790 \mathrm{~nm}$ (blue curve), $825 \mathrm{~nm}$ (green curve), $1050 \mathrm{~nm}$ (pink curve), and the original spherical Au seeds used to grow the nanorods (black curve). For TEM images of these Au NRs cf. Figure VIII.3.9. B) Star-shaped Au NPs with $\lambda_{S P R}=690 \mathrm{~nm}$ (cf. §II.5). For TEM images of these NPs cf. Figure VIII.3.10.

The UV/vis absorption spectrum of NPs may also change in dependence of the surface capping, as well as on the solvent in which the NP are suspended. This is demonstrated in Figure IX.1.9.
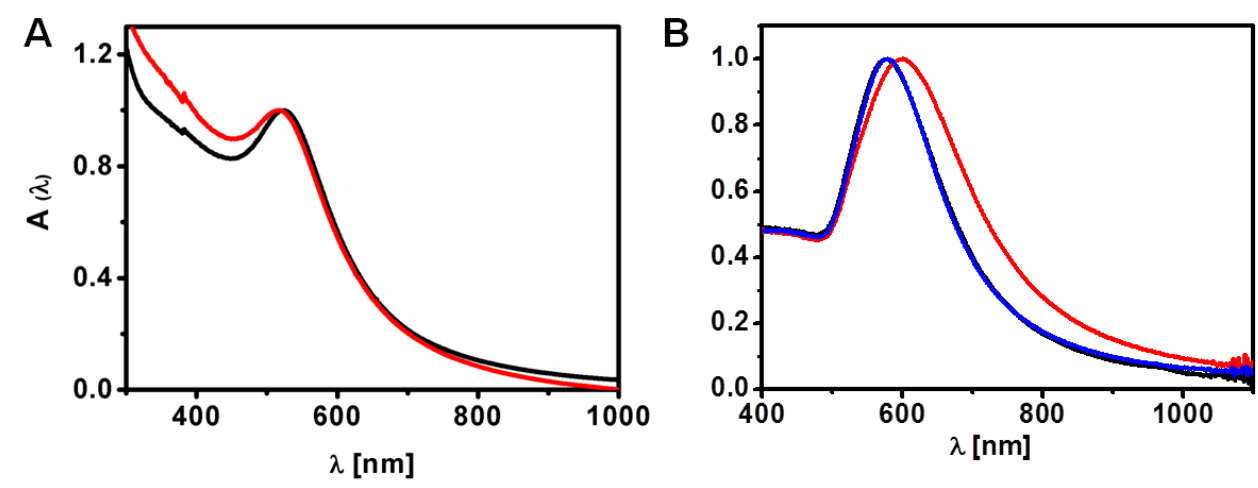

Figure IX.1.9. Normalized UV/vis absorption spectrum of Au NPs capped with different surface coating and suspended in different solvents. A) Au NPs with core diameter of $\approx 4 \mathrm{~nm}$ (cf. §l.2), either capped with DDT and suspended in chloroform (black curve), or after overcoated with PMA (cf. \&VI.3) and suspended in water (red curve). B) Au NPS with core diameter of $\approx 100 \mathrm{~nm}$ (cf. §ll.2) either with the original citrate capping as recorded in water (black) or after their phase transfer to chloroform by ligand exchange with dodecylamine and PEG (cf. §IV.3) (red curve), and finally after re-transfer to water 
by PMA coating (cf. §VI.3) (blue curve). The black and blue curves are almost superimposed in $B$.

Quantum dots have a characteristic absorption peak in their spectrum due to the formation of electron-hole pairs (excitons) upon absorption ${ }^{102-105}$. In the case of semiconductor NPs with very sharp size distribution, multiple exciton peaks can be observed $^{106-108}$. The absorption spectra of CdSe/ZnS and CdS NPs are shown in Figure IX.1.10.
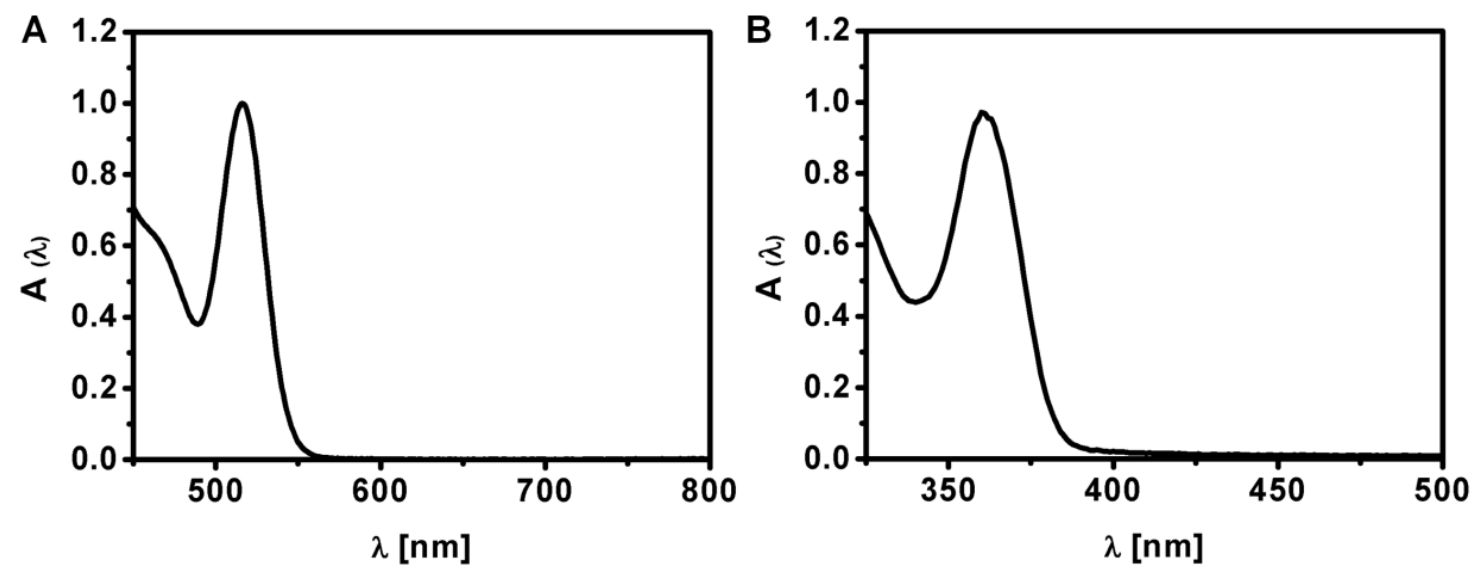

Figure IX.1.10. A) Normalized UV/vis absorption spectrum of CdSe/ZnS quantum dots with core diameter of $\approx 4 \mathrm{~nm}$ (cf. §I.7; Figure VIII.3.5) as recorded in toluene with $\lambda_{\max }=$ $516 \mathrm{~nm}$. B) Normalized UV/vis absorption spectrum of CdS quantum dots with core diameter of $\approx 2 \mathrm{~nm}$ (cf. §l.8; Figure VIII.3.6) as recorded in toluene with $\lambda_{\max }=360 \mathrm{~nm}$.

The spectral position of the first exciton peak, which corresponds to the energy gap between the ground states of the valence and conduction band, is extremely size dependent. This is due to the quantum confinement of light-generated electron-hole pairs ${ }^{109-112}$. The smaller a QD becomes, the bigger the energy gap and thus the shorter the wavelength of the first exciton peak will be. Equation I.7.1 correlates the wavelength $\lambda_{\max }$ of the first exciton peak in the absorption spectrum with the core NP diameter $d_{c}$. Values have been listed for several QDs materials ${ }^{28,113}$. Note that in Yu et al. data are only provided for QDs with a single semiconductor core. However, as demonstrated in §I.7, quantum dots can also be grown with several semiconductor shells around the semiconductor core. For example, the values for CdSe NPs in Yu et al. table cannot be directly applied for CdSe/ZnS NPs, as the ZnS shell will shift the absorption peak $\lambda_{\text {abs }}$ to higher wavelength. If this wavelength is used with Equation 1.7.1 the resulting $d_{c}$ for the CdSe core in CdSe/ZnS is overestimated. However, and since the ZnS shell contributes to the size of the inorganic part of the NPs, this error in the calculation is often tolerated. 


\section{IX.2 Determination of NP concentrations from absorption spectra}

The NP concentration of NP suspension $\mathrm{c}_{\mathrm{NP}}$ can be obtained from the Beer-Lambert's law, which correlates the absorption A (from the UV/vis absorption spectrum) with $\mathrm{C}_{\mathrm{NP}}$ as follows:

$$
A(\lambda)=\varepsilon_{N P}(\lambda) \times l_{\text {sol }} \times c_{N P}
$$

Both, the molar extinction coefficient $\left(\varepsilon_{N P}(\lambda)\right)$ and thus the absorption $(A(\lambda))$ depend on the wavelength $\lambda$ and the solvent. $I_{\text {sol }}$ is the path length of the cuvette in which the absorbance has been measured, i.e. the distance that the light passes in the NP suspension during measurements. As mentioned in §IX.1, a blank spectrum of the solvent should be measured first and subtracted from the sample spectrum, in order to eliminate absorption from the cuvette and the solvent. In order to determine the NP concentration $c_{N P}$ of a sample, the absorption $A(\lambda)$ is measured at wavelength $\lambda$ in a cuvette of path length $I_{\text {sol. }}$ It is important to correct the offset of the absorption spectra as detailed in Figure IX.1.1). In case $\varepsilon_{\mathrm{NP}}(\lambda)$ is known, the NP concentration can be calculated according to Equation IX.2.1.

In case of NPs which do not have specific peaks in their absorption spectra (such as $\mathrm{FePt}, \mathrm{Fe}_{3} \mathrm{O}_{4}, \mathrm{Sn}$, etc.), $\varepsilon_{\mathrm{NP}}(\lambda)$ usually is not reported in the literature. In this case the absorption $A(\lambda)$ of a NP suspension is recorded, and then the NP concentration $\mathrm{C}_{\mathrm{NP}}$ in this solution is determined by ICP-MS (cf. §VIII.2). The molar extinction coefficient can then be calculated as $\varepsilon_{N P}(\lambda)=A(\lambda) /\left(c_{N P} \times\left.\right|_{\text {sol }}\right)$. Once $\varepsilon_{N P}(\lambda)$ is known, the NP concentration in following samples can be determined by absorption instead of ICP-MS analysis, which is less time consuming and cheaper.

For plasmonic NPs or QDs, values for the molar extinction at $\lambda_{\text {SPR }}$ (wavelength of the surface plasmon resonance) or $\lambda_{\max }$ (first exciton peak), respectively, are available in the literature. For Au NPs some molar extinction values $\lambda_{\text {SPR }}$ are given in Table IX.1.1. An extended table has been reported by Haiss et al. ${ }^{114}$ Also in the case of QDs lists of molar extinction values are reported in literature ${ }^{28,115}$. In Figure IX.2.1 an example for the concentration determination of a solution of Au NPs is given. 


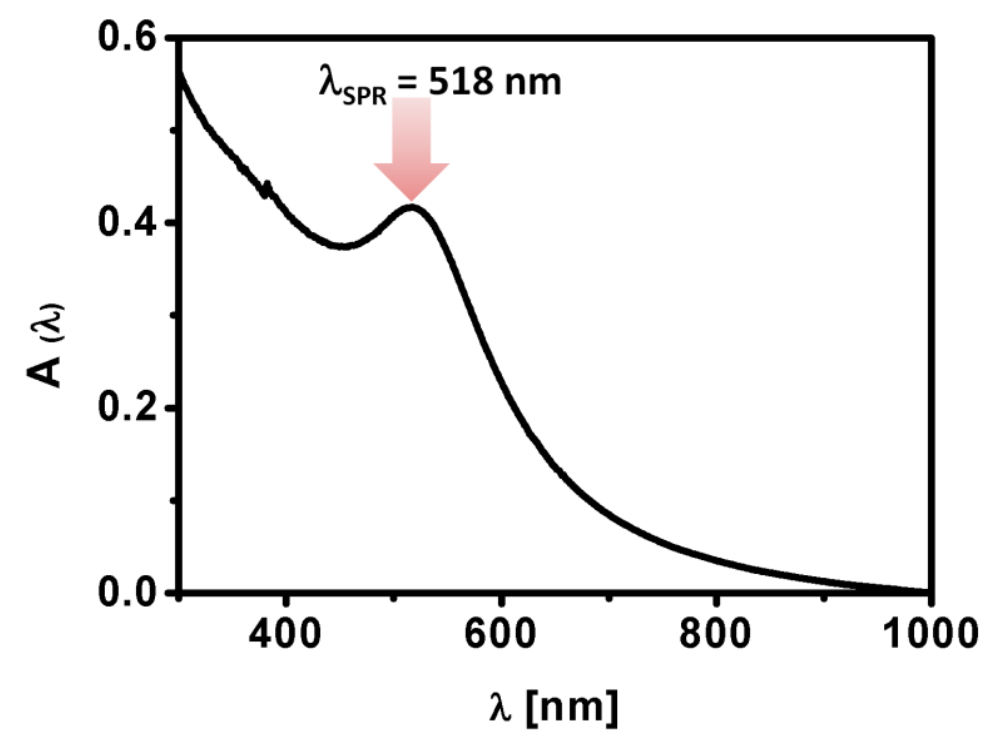

Figure IX.2.1. Absorption spectrum of $\approx 4 \mathrm{~nm}$ Au NPs (cf. §l.2) recorded in chloroform. The absorption at their surface plasmon peak $\lambda_{S P R}=518 \mathrm{~nm}$ is measured to be $A\left(\lambda_{S P R}\right)=$ 0.42 . The path length of the used cuvette was $I_{s o l}=1 \mathrm{~cm}$. The molar extinction coefficient $\varepsilon\left(\lambda_{S P R}\right)=8.7 \times 10^{6} \mathrm{M}^{-1} \times \mathrm{cm}^{-1}$ is obtained from Table IX.1.1. Thus, the concentration of Au NPs in this solution was calculated using Equation IX.2.1 as $c_{N P}=$ $A\left(\lambda_{S P R}\right) /\left(\varepsilon_{N P}\left(\lambda_{S P R}\right) \times I_{\text {sol }}\right)=0.42 /\left(8.7 \times 10^{6} \mathrm{M}^{-1} \times \mathrm{cm}^{-1} \times 1 \mathrm{~cm}\right)=0.048 \times 10^{-6} \mathrm{M}=48 \mathrm{nM}$.

NP concentration values obtained via absorption measurements have to be interpreted with care, as they may be prone to significant errors. With this in mind, published reports should clearly detail calculation of NP concentration stating the used molar extinction values and the selected absorption wavelength.

In case the molar extinction coefficient for a NP sample is recorded at one wavelength $\lambda_{1}$, but the extinction coefficient at another wavelength $\lambda_{2}$ is needed, this can be calculated from the absorption spectrum $A(\lambda)$ according to Equation IX.2.1 as

$$
\varepsilon\left(\lambda_{2}\right)=\varepsilon\left(\lambda_{1}\right) \times A\left(\lambda_{2}\right) / A\left(\lambda_{1}\right)
$$

For homogeneous NPs with characteristic features, such as Au NPs the extinction coefficients of the Au cores are known with relatively good precision ${ }^{114}$. However, these values refer to the absorption related to the NP core, with an assumption that the organic coating does not contribute significantly to the total absorption (as the absorption of the inorganic core is much stronger). Thus, the extinction coefficient for the core is used for the whole NP. In case of thin transparent ligand shells this approximation works well at higher wavelengths (Vis-NIR), but not at lower wavelength (UV) where most organic ligands possess significant absorption. Also in 
case the inorganic part comprises a core and (several) shell(s), such as already mentioned in the case of CdSe/ZnS NPs, the absorption of the shell often is neglected and the extinction values for the core are used for the whole core/shell NP.

The organic shell can be modified intentionally with "absorbers", for example in case organic fluorophores are integrated into the polymer shell around a NP (cf. §VI.2). In this case the absorption spectrum shows the absorption of the inorganic NP core, the absorption of the organic shell (which can be in general neglected), and the absorption of the integrated fluorophores, see Figure IX.2.2. Obviously, it is important to determine the number of fluorophores per NP, for which several methods have been proposed $67,76,116$. One of these methods is based on determining both, the concentration of NP cores $\mathrm{C}_{\mathrm{NP}}$, and the concentration of dyes $\mathrm{C}_{\text {dye }}$ from the UV/vis absorption spectra, leading to the number $\mathrm{N}_{\text {dye/NP }}=\mathrm{c}_{\text {dye }} / \mathrm{c}_{\mathrm{NP}}$ of fluorophores per NP. An example of calculation is shown in Figure IX.2.2.

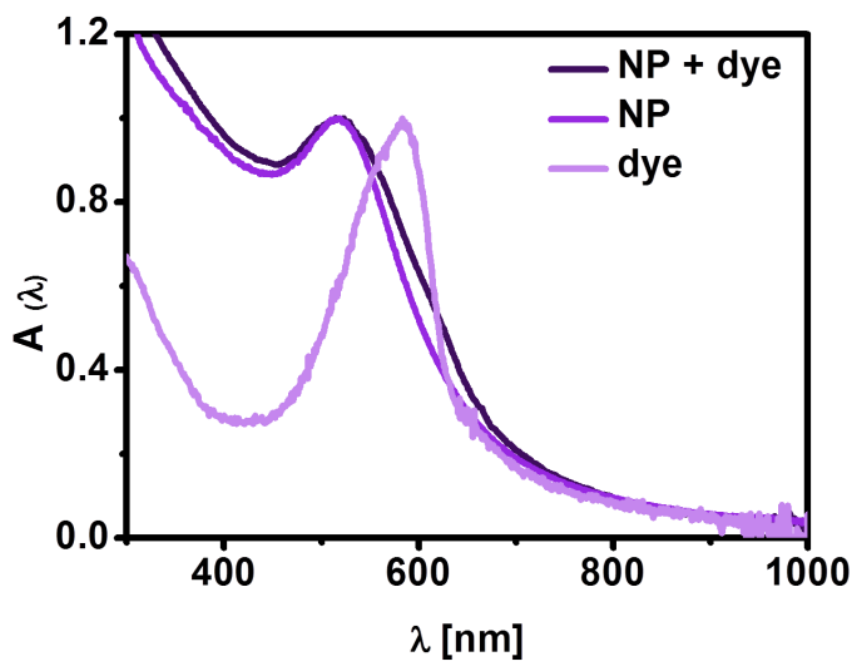

Figure IX.2.2. Normalized absorption spectra of $\approx 4 \mathrm{~nm}$ Au NPs (cf. §l.2; blue curve) recorded after their PMA coating and functionalization with a dye (cresyl violet perchlorate, CV) versus the spectrum of free the dye (red curve). The absorption at surface plasmon peak $\lambda_{S P R}=519 \mathrm{~nm}$ of the NPs is normalized to $A\left(\lambda_{S P R}\right)=1.0$. The absorption of the free dye at their emission maximum $\lambda_{\text {dye, } \max }=583 \mathrm{~nm}$ is normalized to $A\left(\lambda_{\text {dye,max }}\right)=1.0$ as well. To calculate the concentration $c_{\text {dye }}$ of the conjugated dye, the absorption of the NPs at $\lambda_{\text {dye,max }}$ is first subtracted from the absorption of dyemodified NPs at $\lambda_{\text {dye, } \max } \Rightarrow A_{\text {dye }}\left(\lambda_{\text {dye, } \max }\right)=A_{N P+\text { dye }}\left(\lambda_{\text {dye,max }}\right)-A_{N P}\left(\lambda_{\text {dye, } \max }\right)=0.73-0.63=$ 0.1. The molar extinction coefficient of NPS $\varepsilon_{N P}\left(\lambda_{S P R}\right)=8.7 \times 10^{6} \mathrm{M}^{-1} \cdot \mathrm{cm}^{-1}$ is obtained from Table IX.1.1 and the one of CV is $\varepsilon_{\text {dye }}\left(\lambda_{\text {max }}\right)=8.3 \times 10^{4} \mathrm{M}^{-1} \cdot \mathrm{cm}^{-1}$. The path length of the used cuvette was $I_{\text {sol }}=1 \mathrm{~cm}$. Now both concentrations can be calculated according to Equation IX.2.1. For the dye $c_{\text {dye }}=A_{\text {dye }}\left(\lambda_{\text {dye, } \max }\right) /\left(\varepsilon_{\text {dye }}\left(\lambda_{\text {dye, } \max }\right) \times I_{\text {sol }}\right)=0.1 /\left(8.3 \times 10^{4} \mathrm{M}^{-}\right.$ $\left.{ }^{1} \cdot \mathrm{cm}^{-1} \times 1 \mathrm{~cm}\right)=1.21 \times 10^{-6} \mathrm{M}=1.21 \mu \mathrm{M}$. For the $N P S c_{N P}=A_{N P}\left(\lambda_{S P R}\right) /\left(\varepsilon_{N P}\left(\lambda_{S P R}\right) \times I_{s o l}\right)=$ 
$1.0 /\left(8.7 \times 10^{6} \mathrm{M}^{-1} \cdot \mathrm{cm}^{-1} \times 1 \mathrm{~cm}\right)=0.115 \times 10^{-6} \mathrm{M}=115 \mathrm{nM}$. By this the ratio of $N_{\text {dye } / \mathrm{NP}}=$ $c_{\text {dye }} / c_{N P}=1.21 \times 10^{-6} \mathrm{M} / 0.115 \times 10^{-6} \mathrm{M}=10.5$ dye molecules per $N P$ can be estimated.

Typically the absorption spectrum of the dye will overlap with the absorption spectrum of the NP core. Thus, the part of the dye absorption needs to be deconvoluted from the part of the NP core absorption. For this purpose three UV/vis absorption spectra need to be recorded: (i) from solutions of the NPs with integrated fluorophores, (ii) of the NPs alone, and (iii) of the fluorophores alone, cf. Figure IX.2.2 51, 75. The fluorophores typically absorb only in a small spectral band, whereas the NP cores absorb continuously, optionally until a maximum wavelength. Thus the NP core concentration can be determined at a wavelength where the NP core, but not the fluorophore absorbs. This can be directly done by the known molar extinction coefficient $\varepsilon_{\mathrm{NP}}(\lambda)$ at this wavelength by measuring the absorption from the NP spectrum, leading to the concentration of NP cores $C_{N P}$. On the other hand, at the absorption peak of the dye there is typically also absorption of the NP core. Thus, the absorption spectrum of the NP cores only is scaled by multiplication with an appropriate factor to match the absorption spectrum of the NPs with integrated dyes at the parts, where the dyes do not absorb. Then, at the absorption peak of the dye the appropriately scaled absorption of the non-modified NPs is subtracted from the absorption of the NPs with integrated dyes, leading to the absorption of only the dyes. In case the molar extinction coefficient of the dyes $\varepsilon_{\text {dye }}(\lambda)$ at this absorption wavelength is known, then the dye concentration can be calculated using LambertBeer's law:

$$
A(\lambda)=\varepsilon_{\text {dye }}(\lambda) \times c_{\text {dye }} \times I_{\text {sol }}
$$

This methodology works the better the more the characteristic absorption peaks of the NP cores and the more the dyes are spectrally shifted. An example for a calculation is shown in Figure IX.2.2.

Also data from TGA (cf. §VIII.6) can be used for estimating the number of dyes attached per NP. In case the number of polymer units bound the NP ( $\left.\mathrm{N}_{\mathrm{P} / \mathrm{NP}}\right)$ is known, the number of dyes bound per NP ( $\left.N_{\text {dye/NP }}\right)$ can be derived from the amount of dye that has been added per monomer $\left(R_{\text {dye/mon }}\right)$ :

$$
\mathrm{N}_{\text {dye/NP }}=\mathrm{R}_{\text {dye } / \text { mon }} \times \mathrm{N}_{\mathrm{P} / \mathrm{NP}}
$$

In the case of the PMA-Au NPs with $d_{c}=4.3$ as discussed in $\S$ VIII.6, addition of dye to $2 \%$ of the anhydride rings $\left(R_{\text {dye/mon }}=0.02\right)$ leads to $N_{\text {dye/NP }}=0.02 \times 270=54$. This value can now be compared to the values determined with other methods. In Kaiser et al. ${ }^{67}$ 
Evidot ${ }^{\circledR} 540 /$ Catskill Green QDs $\left(d_{c}=2.7 \pm 0.4 \mathrm{~nm}\right.$ determined by TEM, value provided by company: $2.6 \mathrm{~nm}$ ) were modified with $2 \%$ dye $\left(R_{\text {dye/mon }}=0.02\right)$ and the value $N_{\text {dye/NP }}$ $=7 \pm 0.7$ was determined from time resolved photoluminescence spectra. Assuming that the number of dye molecules per NP scale with the effective surface area $A_{\text {eff, }}$ this value can be compared to the TGA data of Au: $N_{\text {dye/NP(1) }}=$ $N_{\text {dye/NP(2) }} x\left(\left(R_{P / A r e a(1)} X A_{\text {eff(1) }}\right) /\left(R_{P / A r e a(2)} x A_{\text {eff(2) }}\right)\right)=N_{\text {dye/NP(2) }} x\left(R_{P / A r e a(1)} /\left(R_{P / A r e a(2)}\right) x\left(\left(d_{c(1)}+\right.\right.\right.$ $\left.\left.\left.2 x\right|_{\text {ligand(1) })}\right) /\left(d_{c(2)}+\left.2 x\right|_{\text {ligand(2) }}\right)\right)^{2}$. In both cases the same amount of polymer per surface area had been added, $R_{P / l i g a n d(1)}=R_{P / l i g a n d(2)}=100 \mathrm{~nm}^{2}$, but the diameters are different. In the case of CdSe $/ Z n S d_{c(1)}=2.7 \mathrm{~nm}, l_{\text {ligand(1) }}=1.2 \mathrm{~nm}$, and in the case of Au: $d_{c(2)}=4.3$ $\mathrm{nm}, l_{\text {ligand(2) }}=1.0 \mathrm{~nm}$. Thus leads to $\mathrm{N}_{\text {dye/NP(1) }}=\mathrm{N}_{\text {dye/NP(2) }} \cdot\left(\left(\mathrm{d}_{\mathrm{c}(1)}+\left.2 \mathrm{x}\right|_{\text {ligand(1) })}\right) /\left(\mathrm{d}_{\mathrm{c}(2)}+\right.\right.$ $\left.\left.\left.2 x\right|_{\text {ligand }(2)}\right)\right)^{2}=54 x((2.7 \mathrm{~nm}+2 \times 1.2 \mathrm{~nm}) /(4.3 \mathrm{~nm}+2 \times 1.0 \mathrm{~nm}))^{2}=54 \times(5.1 / 6.3)^{2} \approx 35$. Thus, TGA analyses leads to a value of $\mathrm{N}_{\text {dye/NP }}=35$ dye molecules per CdSe/ZnS NP, as compared to the optically determined value of $\mathrm{N}_{\text {dye/NP }}=7$. For this reason, in order to estimate the error, it is convenient to use several methods in parallel for the determination of $\mathrm{N}_{\text {dye/NP. }}$.

\section{IX.3 Fluorescence spectroscopy}

NPs can be fluorescent for two reasons. First, NPs may be intrinsically fluorescent, such in the case of QDs ${ }^{117}$, upconverting NPs ${ }^{118}$, ultra-small Au ${ }^{119}$ or Ag clusters ${ }^{120}$. Examples for fluorescence spectra of CdSe/ZnS and CdS NPs are shown in Figures IX.3.1 and IX.3.2, respectively. Second, organic fluorophores can be integrated in the organic surface coating ( $c f . \S \mathrm{VI} .2$ ) around non-fluorescent inorganic NP cores. An example is shown in Figure IX.3.3. In this case the underlying inorganic NP core may affect the fluorescence emission of the organic fluorophores. In case of plasmonic NPs the fluorescence of adjacent dyes may be quenched ${ }^{121}$, and in the case of QDs, fluorescence resonance energy transfer (FRET) may occur with the linked-dyes ${ }^{75,79,122}$. In both cases the effects strongly depend on the distance between the organic fluorophores and the NP cores. 


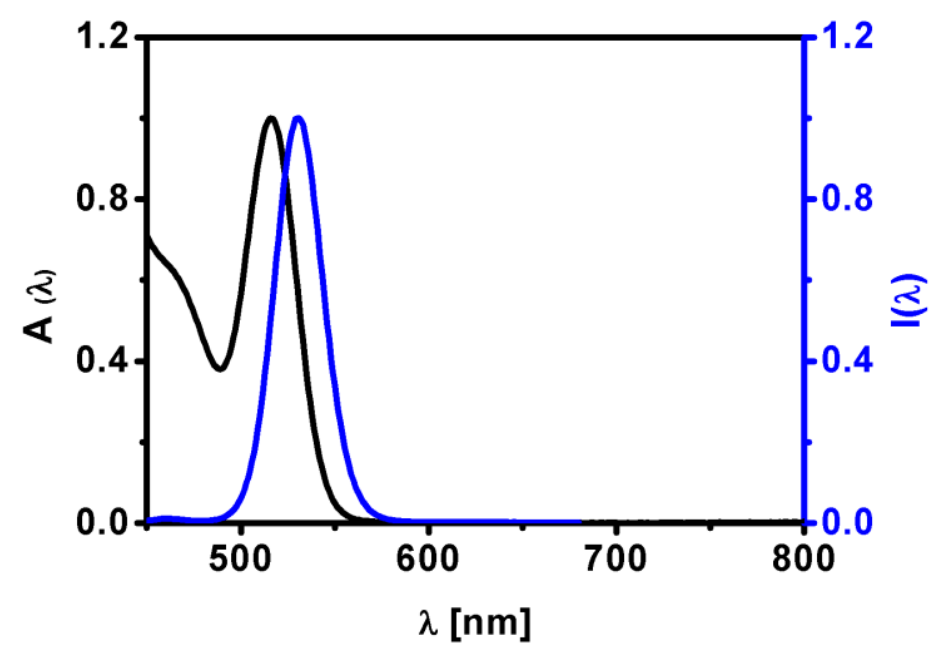

Figure IX.3.1. Normalized absorption spectrum $A(\lambda)$ (black solid line, cf. Figure IX.1.10A) and emission spectrum I( $\lambda$ ) (blue solid line) of hydrophobic CdSe/ZnS NPs $\left(d_{c} \approx\right.$ $4 \mathrm{~nm}, c f . \S(.7)$ in toluene. The absorption and emission peaks are at $\lambda_{a b s} \approx 516 \mathrm{~nm}$ and $\lambda_{\text {em }} \approx 530 \mathrm{~nm}$ respectively, correspond to CdSe/ZnS NPs of $\approx 4 \mathrm{~nm}$.

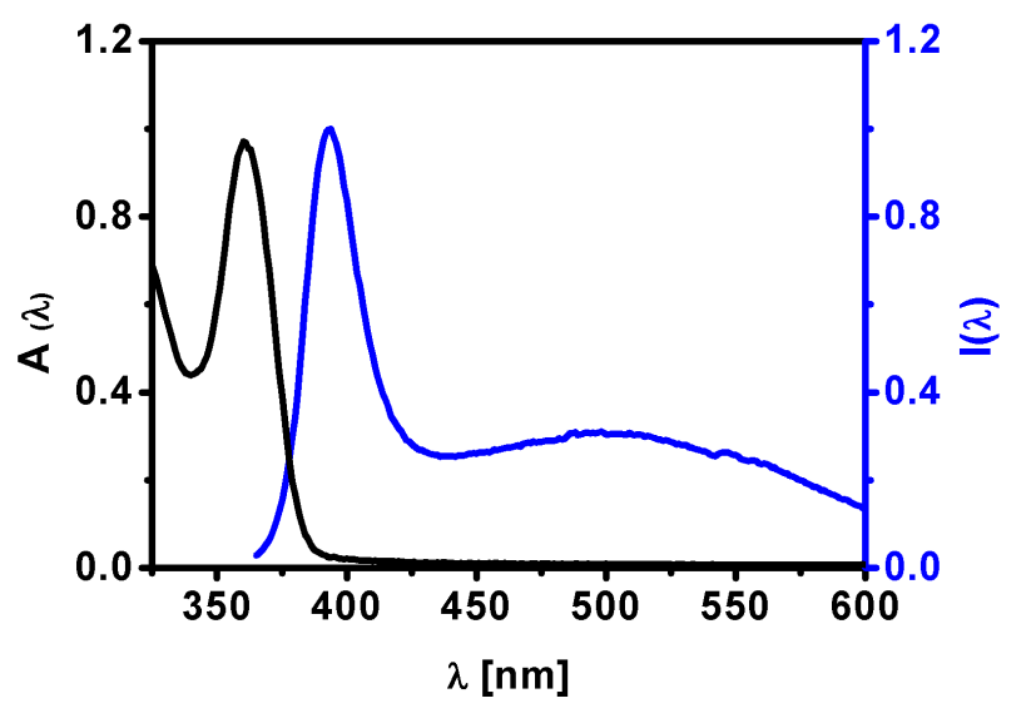

Figure IX.3.2. Normalized absorption spectrum $A(\lambda)$ (black solid line, cf. Figure IX.1.10B) and emission spectrum I( $\lambda$ ) (blue solid line) of hydrophobic CdS NPs $\left(d_{c} \approx 2 \mathrm{~nm}, c f . \S 1.8\right)$ in toluene. The absorption and emission peaks, respectively at $\lambda_{a b s} \approx 360 \mathrm{~nm}$ and $\lambda_{e m} \approx$ $390 \mathrm{~nm}$ correspond to CdS NPs of $\approx 2 \mathrm{~nm}$. 


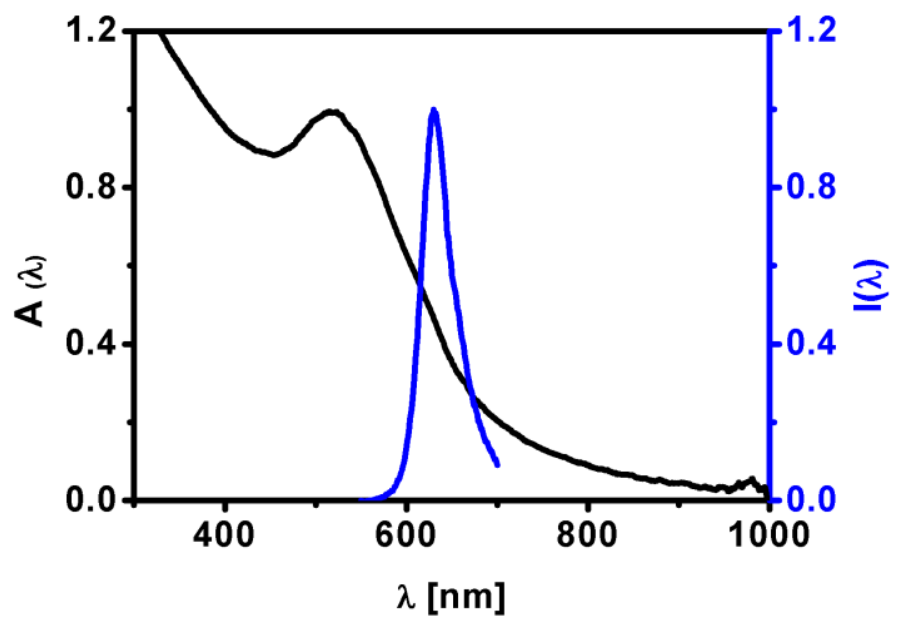

Figure IX.3.3. Normalized absorption spectrum $A(\lambda)$ (black solid line, cf. Figure IX.2.2) and emission spectrum $I(\lambda)$ (blue solid line) of hydrophilic Au NPs coated with PMA modified with cresyl violet $(C V)\left(d_{c} \approx 4 \mathrm{~nm}, c f\right.$. \&l.2) as recorded in water. The absorption and emission peaks are at $\lambda_{a b s} \approx 519 \mathrm{~nm}$ and $\lambda_{e m} \approx 630 \mathrm{~nm}$, respectively.

\section{IX.4 Determination of quantum yields}

A major characteristic of fluorescent NPs is their quantum yield $\left(\Phi_{\mathrm{F}}\right)$. This feature represents the ratio of the number of photons emitted through fluorescence to the number of absorbed photons. In other words, the quantum yield describes the probability that a photo-excited state gets relaxed by fluorescence rather than by another, non-radiative mechanism. As photons may be emitted in all directions, in principle, a $4 \pi$-detection geometry is required to detect absolute quantum yields ${ }^{123}$, 124. Instead, often relative quantum yields are measured in standard $90^{\circ}$ detection geometry. For this purpose fluorescence intensities of a dilution series of the sample (s) and a dilution series of a reference fluorophore (ref) with known absolute quantum yield $\Phi_{\text {ref }}$ are determined. From the slopes $\Delta \mathrm{l}_{\text {int }} / \Delta \mathrm{A}_{\lambda}$ of the integrated emission ( $\mathrm{I}_{\text {int }}$ ) versus absorption $\left(A_{\lambda}\right)$ curves of the sample and the reference fluorophore, the absolute quantum yield of the NP sample can be calculated, $c f$. Equation IX.4.1. A good description of this methodology is detailed by Horiba Jobin Yvon ${ }^{125}$.

$$
\frac{\Phi_{\mathrm{s}}}{\Phi_{\mathrm{ref}}}=\frac{\left(\Delta \mathrm{I}_{\mathrm{int}} / \Delta \mathrm{A}_{\lambda}\right)_{\mathrm{s}}}{\left(\Delta \mathrm{I}_{\mathrm{int}} / \Delta \mathrm{A}_{\lambda}\right)_{\mathrm{s}}} \cdot \frac{\mathrm{n}_{\mathrm{s}}^{2}}{\mathrm{n}_{\mathrm{ref}}^{2}}
$$

$\left[\Phi_{s}, \Phi_{\text {ref }}\right],\left[\left(\Delta \mathrm{l}_{\text {int }} / \Delta \mathrm{A}_{\lambda}\right)_{s},\left(\Delta \mathrm{l}_{\text {int }} / \Delta \mathrm{A}_{\lambda}\right)_{\text {ref }}\right]$, and $\left[\mathrm{n}_{\mathrm{s}}, \mathrm{n}_{\text {ref }}\right]$ refer to the quantum yield, slope of the integrated emission versus absorption curve, and refractive index of the sample and reference fluorophore, respectively. An example for determining the quantum yield of Au NPs with attached fluorophore is provided in Figure IX.4.1 ${ }^{88}$. 
To demonstrate how to determine the quantum yield of NPs $\left(\Phi_{\mathrm{NP}}\right)$, hydrophobic $\mathrm{Au}$ NPs $\left(d_{c} \approx 4 \mathrm{~nm}\right.$ ) were synthetized ( $c f$. §I.2.) and coated with PMA modified with $\mathrm{R}_{\text {dye/mon }}$ = $2 \%$ tetramethylrhodamine 5-(and-6-) carboxamide cadaverine (TAMRA, \#AS-81506, Anaspec) (cf. $\S \mathrm{VI}$ ). After their purification using gel electrophoresis (the process has been repeated twice) ( $c f$. VII.4), the NPs were concentrated, washed with Milli-Q water and filtered by passing them through a syringe membrane filter $(0.22 \mu \mathrm{m}$ pore size) (cf. VII.2). Cresyl violet perchlorate (CV) dissolved in methanol was chosen as reference dye with a known quantum yield $\Phi_{\mathrm{CVP}}=0.54 \pm 0.03^{126}$.

To determine the quantum yield first a dilution series of both samples (NPs in water, $\mathrm{CV}$ in methanol) were generated. Dilution was quantified by measuring the absorption $A_{\lambda}$ of the samples at the excitation wavelength $\lambda_{\text {exc }}=520 \mathrm{~nm}$ of TAMRA. Note that for the NP sample as for the reference dye absorption needs to be detected at the same wavelength. Ideally, the excitation wavelength of the reference dye should be similar to the fluorescent NP sample. The highest concentration of the absorption series should not exceed an optical density of 0.1 , in order to avoid reabsorption and selfquenching of fluorescence, which may occur at high fluorophore concentrations. For each sample within the dilution series the integrated fluorescence emission $I_{\text {int }}$ was determined (i.e. the intensity of the whole fluorescence peak was counted), using the excitation wavelength $\lambda_{\text {exc }}$ at which the absorption $A_{\lambda}$ had been determined before. All fluorescence emission measurements must be made with the same settings of the fluorometer. The recorded absorption $A(\lambda)$ and emission spectra $I(\lambda)$ of the dilution series are shown in Figure IX.4.1. From these curves the respective absorption $A_{520}$ at $\lambda_{\text {exc }}=520 \mathrm{~nm}$ was determined. The integrated emission $\mathrm{I}_{\text {int }}$ was obtained as the sum of all fluorescence emission counts in the spectral range from $530 \mathrm{~nm}$ to $750 \mathrm{~nm}$. The resulting $\mathrm{l}_{\text {int }}\left(\mathrm{A}_{520}\right)$ curves show the linear relation between absorbance and integrated fluorescence intensity. From these graphs the slopes $\Delta \mathrm{I}_{\text {int }} / \Delta \mathrm{A}_{520}$ were derived. In the case of the NP and the CV sample $(5.11 \pm 10.4) \times 10^{8} \mathrm{cps}$ and $(110 \pm 1.31) \times 10^{8} \mathrm{cps}$ were obtained (cps = counts per second). In order to calculate the quantum yield of the NP sample according to Equation IX.4.1, the refractive indexes $\mathrm{n}_{\mathrm{H} 2 \mathrm{O}}=1.333$ for water and $\mathrm{n}_{\text {methanol }}=1.3288$ for methanol at $20^{\circ} \mathrm{C}$ were used, yielding $\Phi_{\mathrm{NP}}=\Phi_{\mathrm{CVP}} \cdot \frac{\left(\Delta \mathrm{I}_{\text {int }} / \Delta \mathrm{A}_{520}\right)_{\mathrm{NP}}}{\left(\Delta \mathrm{I}_{\mathrm{int}} / \Delta \mathrm{A}_{520}\right)_{\mathrm{CV}}}$. $\frac{n_{\mathrm{H} 2 \mathrm{O}}^{2}}{\mathrm{n}_{\text {methanol }}^{2}}=0.025 \pm 0.008$. The error was estimated using Gaussian error propagation. 

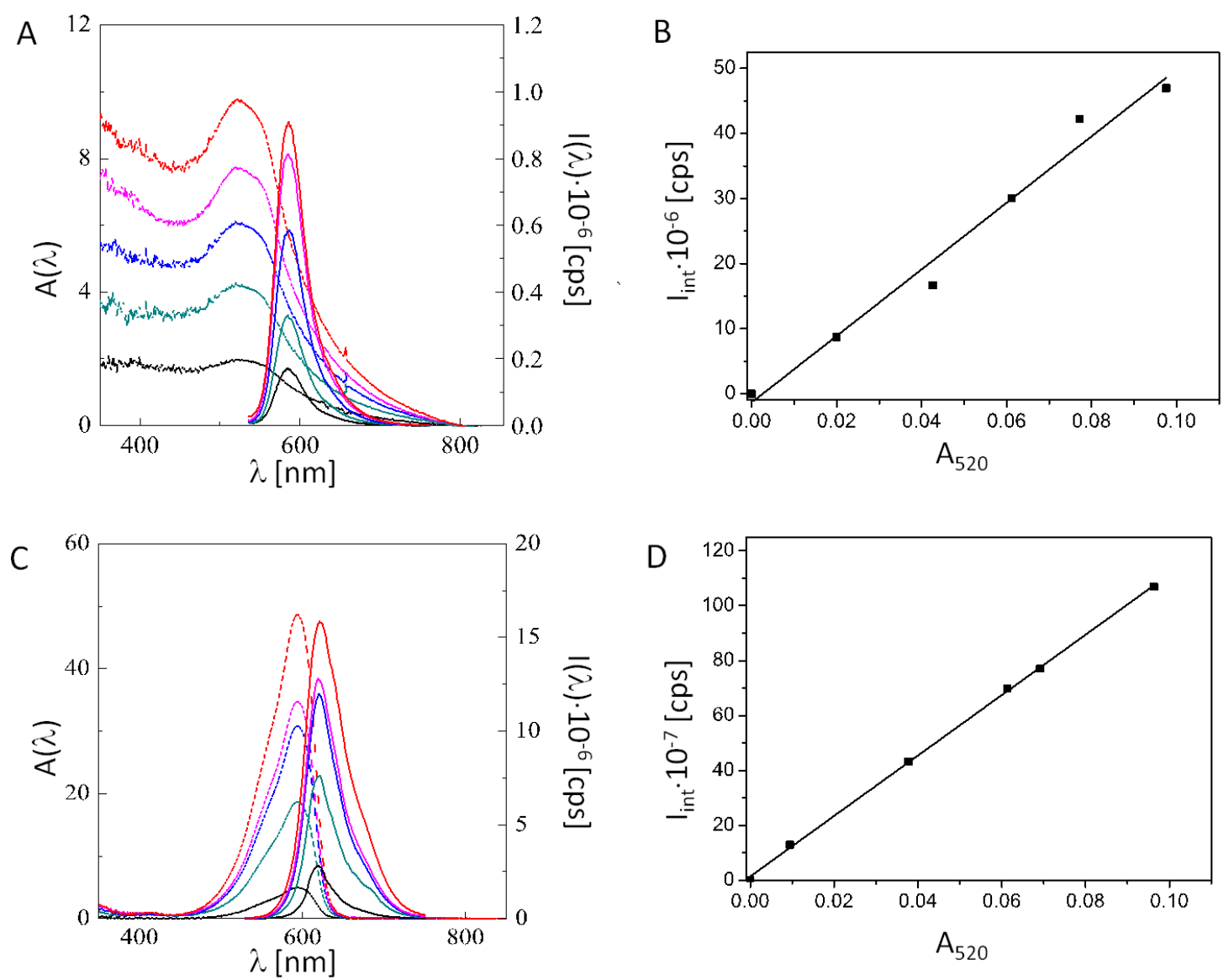

Figure IX.4.1. Determination of the quantum yield $\Phi_{N P}$ of TAMRA modified Au NPs (top row) and $C V$ (bottom row). The graphs on the left side $(A, C)$ represent the absorption spectra $A(\lambda)$ (dotted line, left $y$-axis) and emission spectra I( $\lambda)$ (full line, right $y$-axis) recorded at excitation wavelength $\lambda_{\text {exc }}=520 \mathrm{~nm}$ for dilution series of the Au NPs and CV. From each absorption spectrum the absorption $A_{520}$ at $520 \mathrm{~nm}$ was determined. From each emission spectrum at $520 \mathrm{~nm}$ excitation, the integrated fluorescence intensity $I_{\text {int }}$ was determined as sum of all emitted fluorescence from $530 \mathrm{~nm}$ to 750 $n m$. 


\section{Characterization of NPs' colloidal properties}

\section{X.1 UV/vis absorption spectroscopy}

\section{$X .2$ Dynamic light scattering}

\section{X.3 Laser Doppler anemometry}

\section{$\mathrm{X} .4 \mathrm{pH}$ titration}

\section{X.1 UV/vis absorption spectroscopy}

UV/vis absorption spectrum provides a fast and simple quality control measure for the colloidal stability of NPs. Several types of NPs, such as plasmonic NPs and QDs possess characteristic peaks in their absorption spectrum (cf. IX.2) that can provide important information regarding size, shape, surface chemistry and aggregation state. For example, aggregation of Au NPs is typically associated with a red shift of the SPR $\left(\lambda_{S P R}\right)$ to higher wavelengths and a significant spectral broadening. Most isotropic inorganic NPs absorb in the UV/vis and not in the near IR, unless they have been designed for this purpose (e.g. rod-shaped Au NPs, Au nanostars, IR emitting QDs, etc.). Thus, absorption in the range where NPs do not typically absorb, indicates NP aggregation. "Absorption" in this case is mainly light scattering due to NP agglomeration. Figure X.1.1 shows the red shift and broadening of the plasmon absorption spectrum of $\mathrm{Au}$ NPs upon salt-induced aggregation.

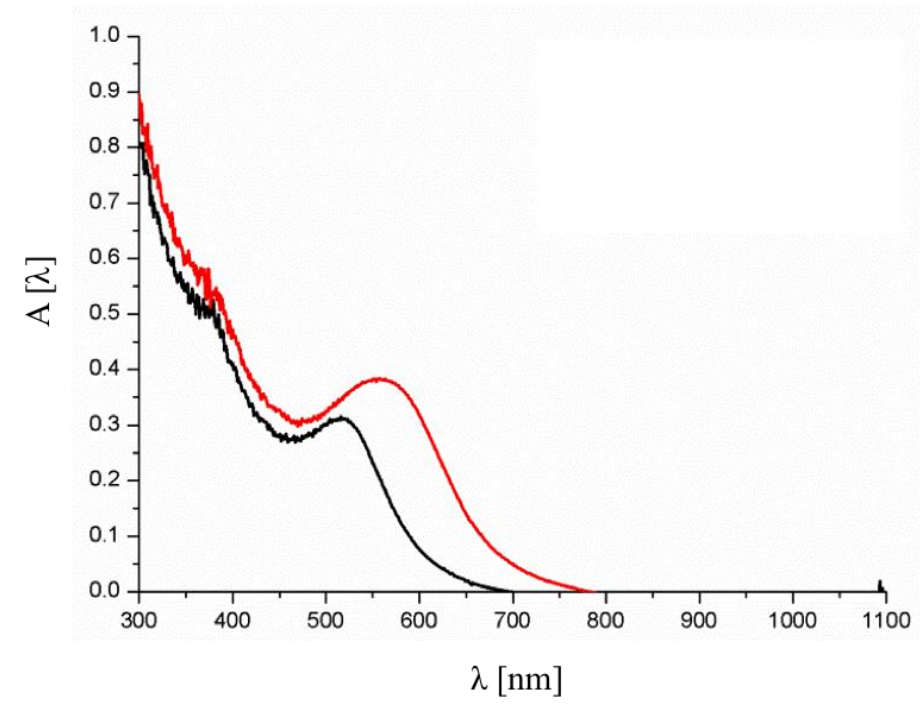

Figure X.1.1. Absorption spectra of $5 \mathrm{~nm}$ core diameter Cit-Au NPs (purchased from $B B I)$ as recorded in water (black curve) and in water supplemented with $\mathrm{NaCl}$ (red curve). The red shift of the SPR peak to higher wavelength and associated peak broadening is clear upon the addition of $\mathrm{NaCl}$ due to NP aggregation and plasmon coupling effect. 
Colloidal stability is a critical property for NPs dispersions, which can be maintained by ensuring repulsive force (either an electrostatic or a steric repulsion) that overweights the attractive van der Waals forces between NPs ${ }^{5}$. For example, Cit-Au NPs are stabilized through electrostatic repulsion due to the presence of physically adsorbing citrate ions on the surface of Au NPs (zeta potentials of these NPs are typically negative $\sim-30 \mathrm{mV}$ ). When salt is added, electrostatic forces are screened by counter ion effects and electrostatic double layer compression, and thus NPs tend to aggregate. The higher the charge density of the NP surface, the less prone the NPs are to aggregation. Note, that the surface charge of NPs may depend on the $\mathrm{pH}(c f . \S X .4)$. NPs that are stabilized by steric repulsion are typically less prone to aggregation upon the addition of salt. In various biological applications, NPs are expected to be dosed into media with physiological salt levels $(150 \mathrm{mM} \mathrm{NaCl})$ and thus colloidal stability should be carefully evaluated.

By titrating the NP suspension against increasing concentrations of $\mathrm{NaCl}$, one can observe the aggregation state by UV/vis absorption measurements. Such measurements in particular allow for observing the $\mathrm{NaCl}$ concentration at which the colloidal stability of the NPs is lost. Figures X.1.2 and X.1.3 show the colloidal stability of Au NPs or Ag NPs with different surface coatings, as judged by their UV/vis absorption spectra upon titration with $\mathrm{NaCl}$ aqueous solution. As agglomeration is time dependent, these measurements were carried out directly after exposure of the NPs to salt, and were then repeated after $24 \mathrm{~h}$. The absorption spectra were measured over a range of 300 to $1100 \mathrm{~nm}$ with an Agilent Technologies $8453 \mathrm{UV} / \mathrm{vis}$ spectrometer.

In most cases after $24 \mathrm{~h}$ the absorption peaks became broader and smaller. The increment in the peak width was due to agglomeration. The reduction in absorption intensity was due to the severe degree of aggregation that decreased the number of individually dispersed NP due to the significant NP loss via precipitation out from the suspension. Collectively, data in Figure X.1.2 and X.1.3 suggest that PMA is superior to MPA and MUA in protecting NPs against salt-induced aggregation. 

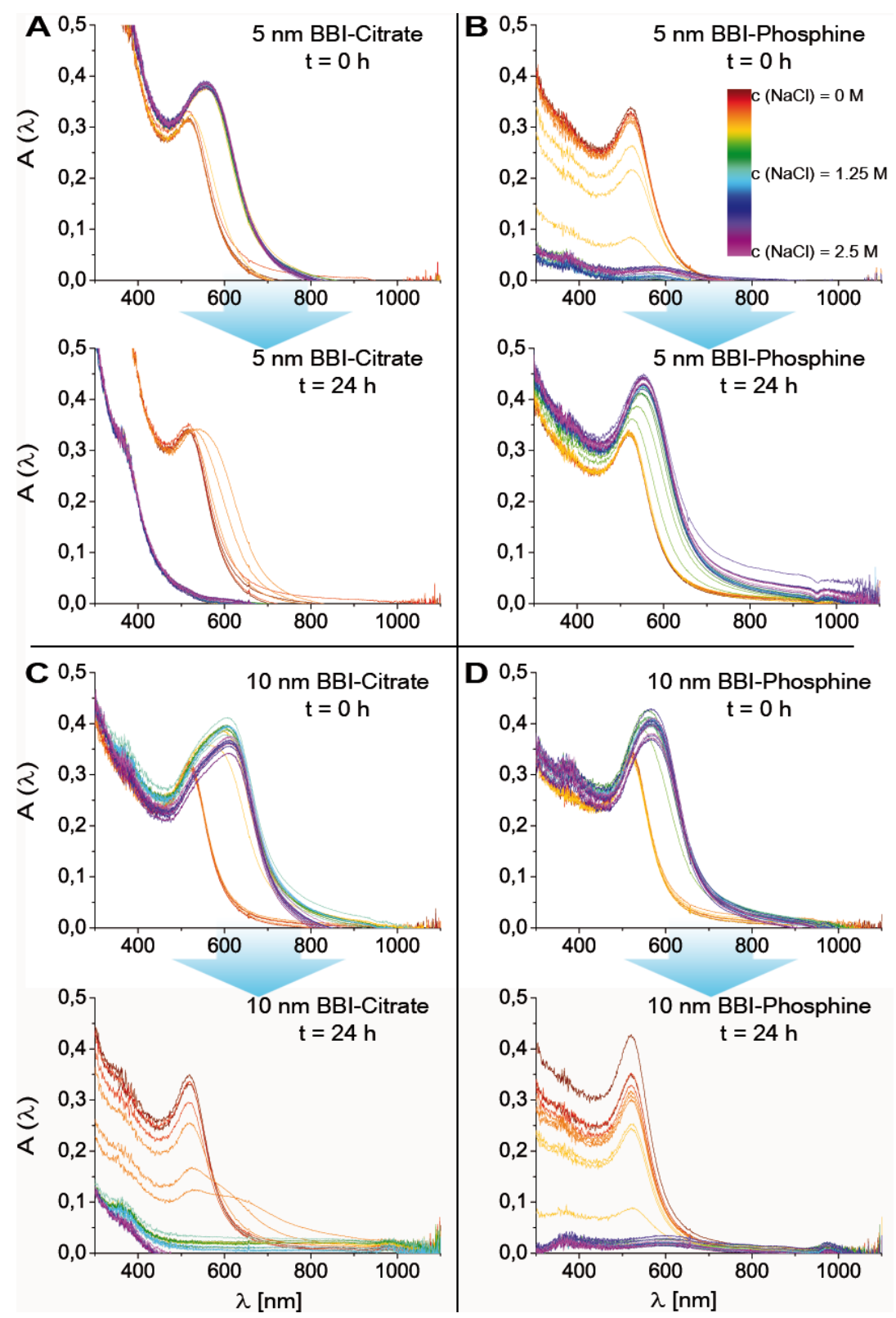

Figure X.1.2. Absorption spectra recorded for different Au NPs in water in the presence of different concentrations of $\mathrm{NaCl}$, recorded after 0 and $24 \mathrm{~h}$, with $c_{N P} \approx 12 \mathrm{nM}$. The $\mathrm{NaCl}$ concentrations were in the range of 0.0 to $2.5 \mathrm{M}$ as illustrated using a color map. Cuvettes with freshly mixed solutions were measured at $t=0 \mathrm{~h}$ before incubating over night at $R T$ and measured again after $t=24 \mathrm{~h}$. A) $\approx 5 \mathrm{~nm}$ core diameter Cit-Au NPs purchased from BBI). B) $\approx 5 \mathrm{~nm}$ core diameter Cit-Au NPs (purchased from BBI), after ligand exchange to bis(p-sulfonatophenyl)-phenylphosphine (cf. \$lll.2). C) $\approx 10 \mathrm{~nm}$ core diameter Cit-Au NPs (purchased from BBI). D) $\approx 10 \mathrm{~nm}$ core diameter Cit-Au NPS (purchased from BBI), after ligand exchange to bis(p-sulfonatophenyl)-phenylphosphine (cf. §III.2). 


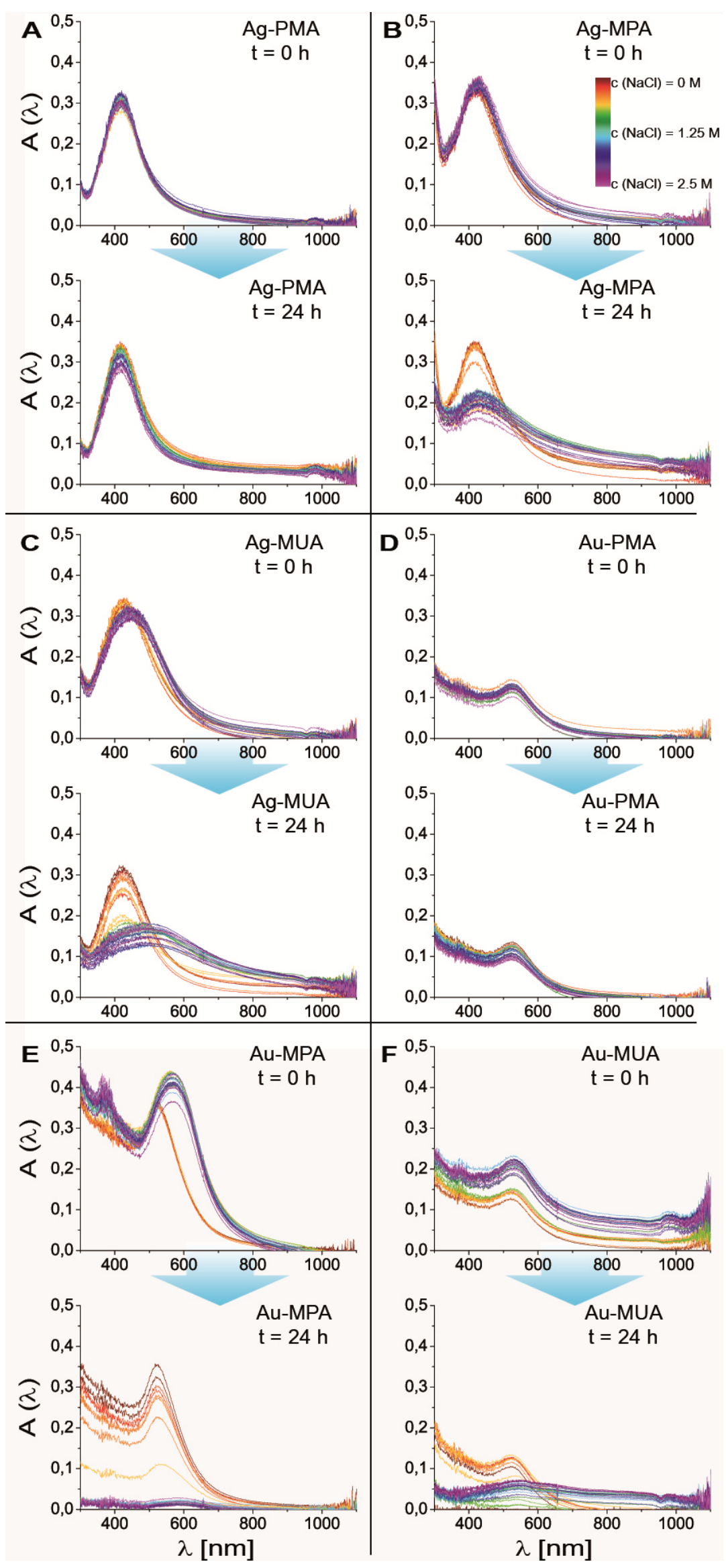


Figure X.1.3. Absorption spectra of different NPs in water in the presence of different concentrations of $\mathrm{NaCl}$ recorded after 0 and $24 h$, with $c_{N P} \approx 12 \mathrm{nM}$. The $\mathrm{NaCl}$ concentrations were in the range of 0.0 to $2.5 \mathrm{M}$ as illustrated using a color map. Cuvettes with freshly mixed solutions were measured at $t=0 \mathrm{~h}$ before incubating over night at $R T$ and measured again after $t=24 \mathrm{~h} . A) \approx 4 \mathrm{~nm}$ core diameter Ag NPs (cf. §l.3) overcoated with PMA (cf. §VI.3). B) $\approx 4 \mathrm{~nm}$ core diameter Ag NPs (cf. §I.3) after ligand exchange with mercaptopropionic acid (MPA; $c f . \S V .2) . C) \approx 4 \mathrm{~nm}$ core diameter Ag NPs (cf. §l.3) after ligand exchange with mercaptoundecanoic acid (MUA; cf. §V.2). D) $\approx 4$ $\mathrm{nm}$ core diameter Au NPs (cf. §I.2) overcoated with PMA (cf. §VI.3). E) $\approx 4 \mathrm{~nm}$ core diameter Au NPs (cf. §l.2) after ligand exchange with mercaptopropionic acid (MPA; cf. $\S V .2)$. F) $\approx 4 \mathrm{~nm}$ core diameter $A u$ NPs (cf. §l.2) after ligand exchange with mercaptoundecanoic acid (MUA; cf. §V.2).

\section{X.2 Dynamic light scattering}

There are several techniques available, which are able to measure the hydrodynamic diameter $d_{h}$ of NPs dispersed in solvent. Hydrodynamic diameters may differ for NPs with different core diameter $d_{c}$, capping agents/polymers, solvent, and aggregation state. For example, identical NPs with a PEG shell exhibit different $d_{h}$ in water versus chloroform due to different hydration/solvation nature and polymer architecture on the NPs in each solvent. In another context, the addition of a high concentration of electrolyte to aqueous suspension containing charged NPs may decrease the $d_{h}$ of these NPs due to the counter ion effect and thus a compressed electric double layer (Debye length) ${ }^{127}$. In addition to measuring the average value of $d_{h}$, it is also important to determine the monodispersity of NP suspensions by measuring the so called polydispersity index (PDI) ${ }^{128}$. The PDI is an unitless parameter that measures the heterogeneity (statistical variation from the average value) in the NPs' hydrodynamic diameter in a sample. Values below 0.05 are normally not obtained, meanwhile values higher than 0.7 indicate that the sample has a very broad size distribution.

There are various techniques to measure hydrodynamic diameters of NPs ${ }^{77,}{ }^{129}$. Dynamic light scattering (DLS) is a simple, fast, and common technique to measure the hydrodynamic diameter of NPs and associated PDI ${ }^{130,131}$. DLS records the temporal fluctuations in scattered light, due to diffusion of NPs in solution. Using an autocorrelation function, the diffusion constant of the NPs then can be derived. By applying the Einstein-Stokes relation and using the measured diffusion constant value, the NPs' hydrodynamic diameter $d_{h}$ can be calculated. Obtaining reliable size 
measurements of NPs of only a few $\mathrm{nm}$ in diameter using DLS is a real challenge, since the scattering signal from these small NPs is close to the noise of the detector.

It is worth to mention that serious error in measuring the absolute size of NPs using DLS may occur and can originate from various reasons such as dirty cuvettes, contaminated solvent, using incorrect values for solvent physical parameters (e.g. viscosity and dielectric constant), improper sample concentration (too high or too low), and unstable samples during measurement. To avoid possible under- or overestimation of NP hydrodynamic size, an internal calibration with reference NPs is essential.

When carrying out DLS analysis, the obtained distributions of hydrodynamic diameters can be plotted in terms of the number of NPs $N\left(d_{h}\right)$ or the intensity of the scattered light $I\left(d_{h}\right)$ as shown in Figure X.2.1. The intensity distribution $I\left(d_{h}\right)$ considers the scattering intensity of each NP fraction. Since larger NPs scatter much more than smaller NPs, the intensity distribution can provide overestimated values of hydrodynamic diameter. For example, a very low level of aggregates in an Au NP suspension will scatter extensively and thus will result in a resolved significant peak (second and third peaks in Figure X.2.1A). Applying Mie's theory, the intensity distribution can be converted to volume or number distributions. To do so, there are several assumptions made, including that all NPs are spherical and homogeneous, which in the case of samples with broad size distribution and/or anisotropic NPs is not valid. In the number distribution, the contribution of large NP populations at low level is very minimal and does not dominate, and thus measured $d_{h}$ values are closer to the $d_{c}$ values as obtained by TEM. As example, in Figure X.2.1A, $d_{h}$ obtained from the intensity distribution (first peak) is $26.07 \pm 12.75 \mathrm{~nm}$ where $d_{h}$ obtained from the number distribution in Figure X.2.1B is $11.71 \pm 3.29 \mathrm{~nm}$, which is closer to the value obtained by TEM $\left(d_{c}=4.3 \mathrm{~nm} \pm 0.4 \mathrm{~nm}\right.$ as per Figure VIII.3.1 plus the addition of the $\mathrm{I}_{\text {ligand }}$ (see Figure VII.5.2. B and C). Commercial DLS set-ups often assume the presence of several NP populations in solution, and thus fits of even one NP population may result in several distinct sizes. For example, in the intensity distribution shown in Figure X.2.1 three peaks are observed, whereas only one peak in the number distribution. In fact, only one NP species is present in solution, small agglomerates are overestimated in the intensity distribution, which then wrongfully identifies three discrete sub-species. 

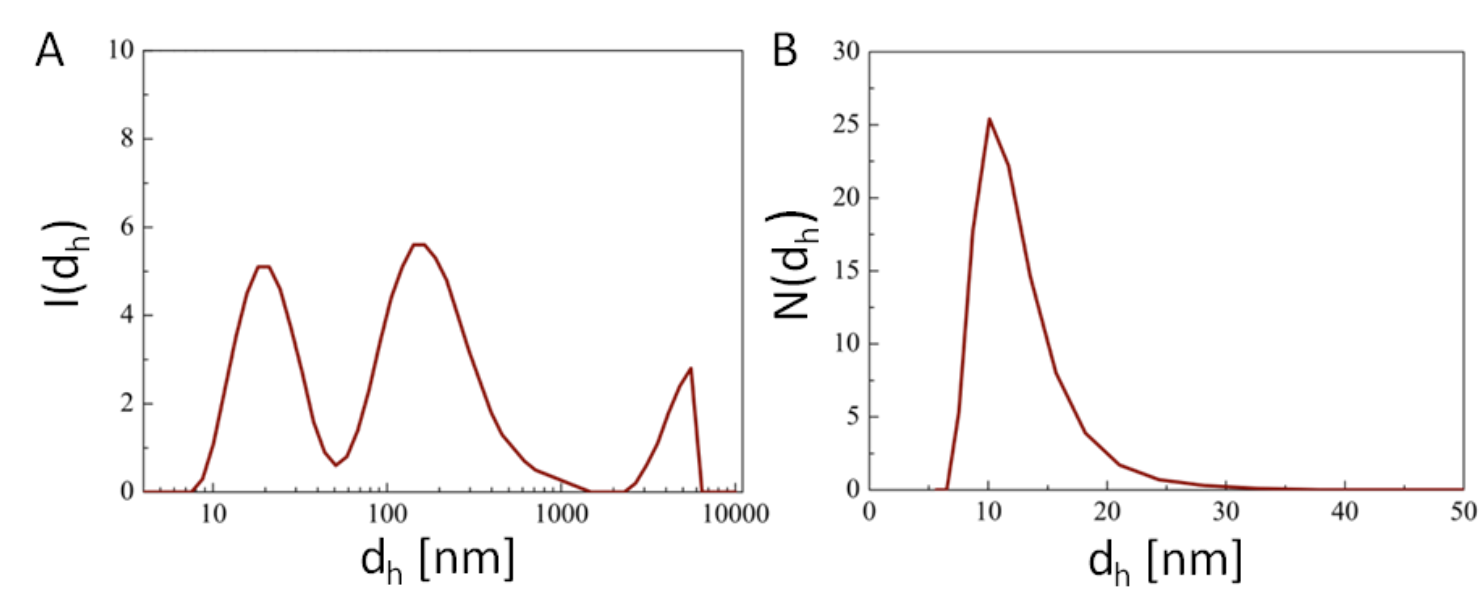

Figure X.2.1. A) DLS histograms of $A U N P s \approx 4 \mathrm{~nm}$ coated with PMA measured in aqueous solution. A) Intensity distribution $I\left(d_{h}\right)$ and $\left.B\right)$ number distribution $N\left(d_{h}\right)$ of the hydrodynamic diameter $d_{h}$.

A significant increase in measured hydrodynamic diameter of NPs as compared to the structural diameter (i.e. $d_{h} \gg d_{c}$ for NPs without the presence of thick organic shell) is usually a strong indication of NP aggregation. In case NPs are aggregated, the measured effective hydrodynamic diameter values describe the diameter of NP agglomerates rather than the diameter of the individual NPs. In Figure X.2.2, aggregation of PMA-Au NPs upon the addition of $\mathrm{NaCl}$ is associated with an increase in $d_{h}$. Such measurement of hydrodynamic diameters is an essential tool to probe the colloidal stability of NPs in solution.

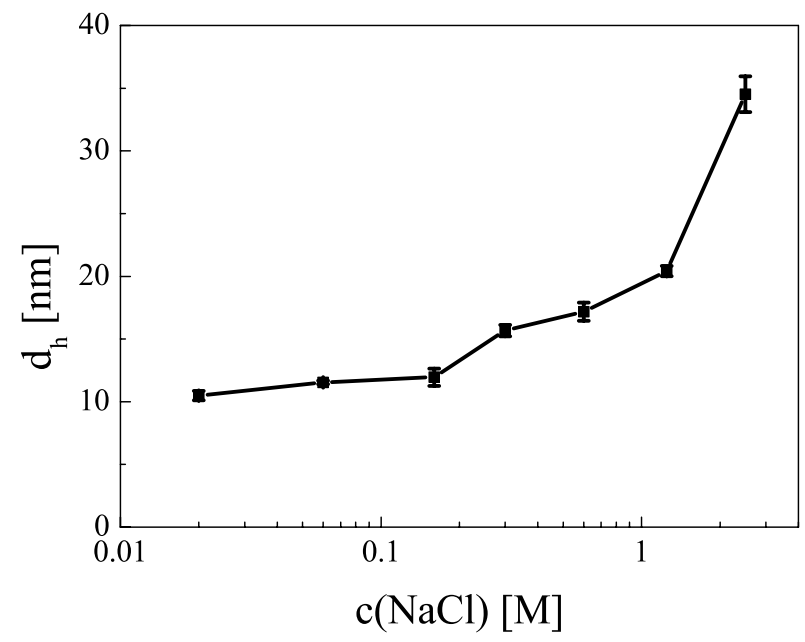

Figure X.2.2. Hydrodynamic diameter $\left(d_{h}\right)$ of $4 \mathrm{~nm} P M A-A u N P s$ as function of $\mathrm{NaCl}$ concentration in the NP suspension.

More examples on the salt-induced aggregation of Ag NPs and Au NPs with differing surface modification and core diameter are provided in Figure X.2.3. Aqueous 
suspension of the NPs (1.0 mL; $\mathrm{c}_{\mathrm{NP}}=25 \mathrm{nM}$ ) received equal volume of $\mathrm{NaCl}$ (aq.) $(1.0 \mathrm{~mL}$; concentration ranges from 0.0 to $2.5 \mathrm{M})$. Obviously the final $\mathrm{NaCl}$ concentration was hereby reduced to half of the original concentration upon mixing it with the same amount of NP stock solution. The hydrodynamic diameter $d_{h}$ was measured immediately after mixing the two solutions and after an $24 \mathrm{~h}$ incubation time at RT (since aggregation is a time-dependent process that can be slow, but significant). DLS histograms ( $\mathrm{d}_{\mathrm{h}}$ versus $\mathrm{C}_{\mathrm{NaCl}}$ ) and real photographs of NP suspensions in the cuvettes are shown in Figure X.2.3, in which agglomeration can be directly observed by visible color change. In most cases aggregation of NPs at high $\mathrm{NaCl}$ concentrations was severe, inducing NP precipitation and sedimentation on the bottom of the cuvette. The results indicated that PMA is superior that MPA or MUA in protecting NPs against salt-induced aggregation. PMA coating resulted in constant hydrodynamic diameter values and suspension color even for high $\mathrm{NaCl}$ concentrations. Addition of $\mathrm{NaCl}$ to MPA or MUA coated NP resulted in increased hydrodynamic diameter values and change in suspension color, indicating NP aggregation. 

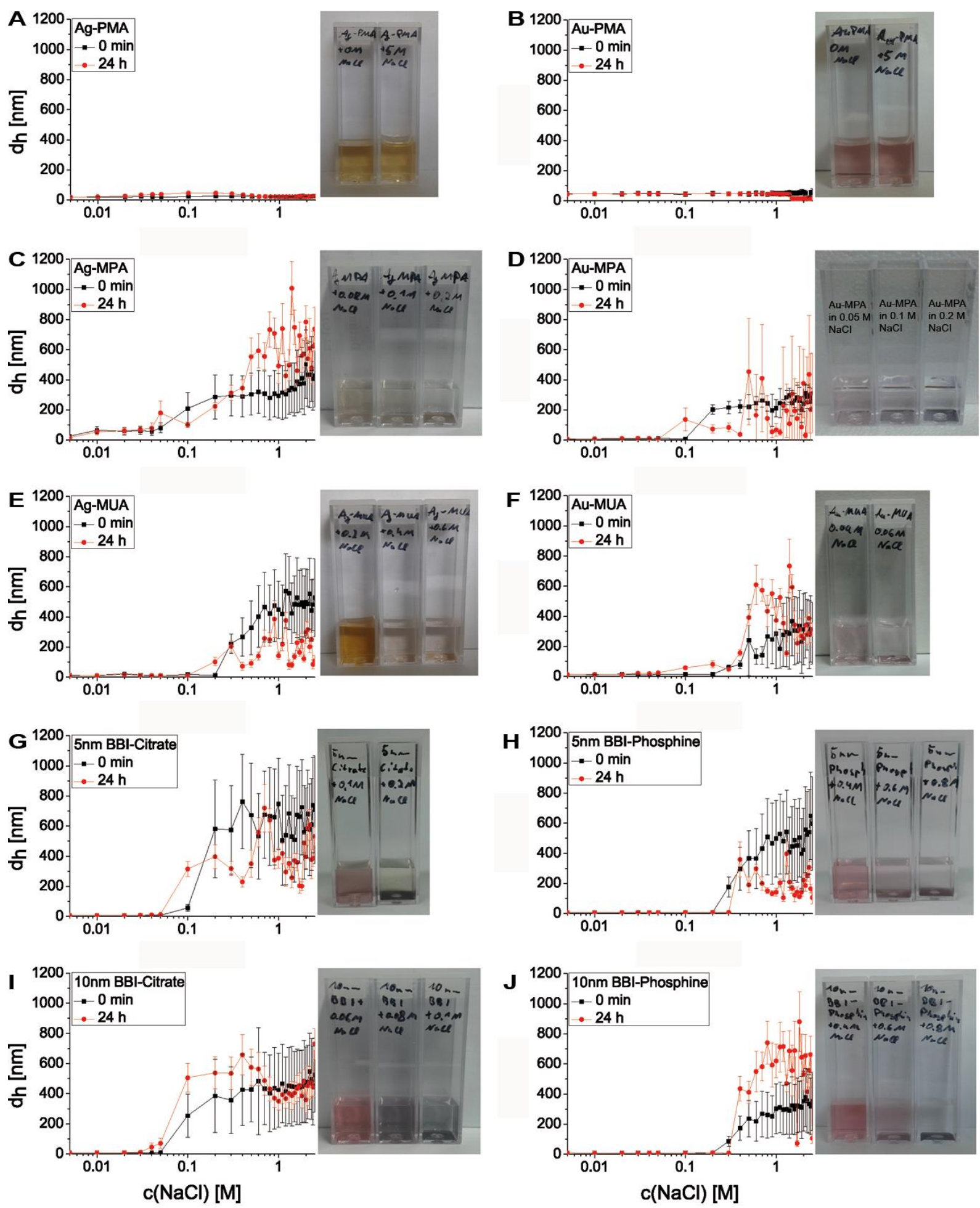

Figure X.2.3. Ag NPs and Au NPs with differing surface modification and core diameter were exposed to different concentrations of $\mathrm{NaCl}$. Hydrodynamic diameter values $d_{h}$ in aqueous solution were measured directly (0 h; ) and $24 h(\bullet)$ after exposure. The behavior of the hydrodynamic diameter $d_{h}$ is plotted against the $\mathrm{NaCl}$ concentration within a range from $C_{\mathrm{NaCl}}$ of 0.0 to $2.5 \mathrm{M}$. The NPs were suspended in Milli-Q water to reach a concentration of $25 \mathrm{nM}$. DLS measurements were done with a Malvern 
Zetasizer nano ZS. The cuvette photos represent the relevant concentrations within the range where agglomeration took place. A) $\approx 4 \mathrm{~nm}$ core diameter Ag NPs (cf. §l.3) overcoated with PMA (cf. §VI.3). B) $\approx 4 \mathrm{~nm}$ core diameter Au NPs (cf. §I.2) overcoated with PMA (cf. §VI.3). C) $\approx 4 \mathrm{~nm}$ core diameter Ag NPs (cf. §l.3) after ligand exchange with MPA (cf. §V.2). D) $\approx 4 \mathrm{~nm}$ core diameter Au NPs (cf. §l.2) after ligand exchange with mercaptopropionic acid (MPA; cf. §V.2). E) $\approx 4 \mathrm{~nm}$ core diameter Ag NPs (cf. §l.3) after ligand exchange with MUA (cf. $\S V .2) . F) \approx 4 \mathrm{~nm}$ core diameter Au NPs (cf. §I.2) after ligand exchange with MUA (cf. $\S V .2)$. G) $\approx 5 \mathrm{~nm}$ core diameter Cit-Au NPs (purchased from BBI). H) $\approx 5 \mathrm{~nm}$ core diameter citrate coated Au NPs (purchased from $B B I)$, after ligand exchange to bis(p-sulfonatophenyl)-phenylphosphine (cf. §III.2). I) $\approx 10 \mathrm{~nm}$ core diameter Cit-Au NPs (purchased from BBI). J) $\approx 10 \mathrm{~nm}$ core diameter citrate coated Au NPS (purchased from BBI), after ligand exchange to bis( $p$ sulfonatophenyl)-phenylphosphine (cf. §III.2).

Similar to NP aggregation upon salt addition, NP aggregation can be induced by changing the suspension $\mathrm{pH}$. While salt screens the electric charge on the NP surface, $\mathrm{pH}$ may suppress ionization and thus decrease the effective surface charge density, resulting in NP aggregation ( $c f . \S X .3$ and $\S X .4$ ). In Figure X.2.4, examples on pH-induced NP aggregation and the dependence of hydrodynamic diameter from solution $\mathrm{pH}$ for anionic charged NPs with different types of surface ligands are shown.

For NP with ligands containing weak acids, such as carboxylate functional groups ($\mathrm{COO}^{-}$), the NP effective surface charge decreases at low $\mathrm{pH}$ (i.e. high concentration of $\mathrm{H}^{+}$) due to protonation of the $-\mathrm{COO}^{-}$groups:

$$
\mathrm{R}-\mathrm{COOH} \leftrightarrow \mathrm{R}-\mathrm{COO}^{-}+\mathrm{H}^{+}
$$

(Equation X.2.1)

This equilibrium is described by the $\mathrm{pK}_{\mathrm{a}}$ value.

$$
\begin{aligned}
& \mathrm{K}_{\mathrm{a}}=\frac{\mathrm{c}\left(\mathrm{R}-\mathrm{COO}^{-}\right) \times \mathrm{c}\left(\mathrm{H}^{+}\right)}{\mathrm{c}(\mathrm{R}-\mathrm{COOH})} \\
& \mathrm{pK}_{\mathrm{a}}=-\log \left(\mathrm{K}_{\mathrm{a}}\right)
\end{aligned}
$$

Equations X.2.2 and X.2.3 can be combined to the Henderson-Hasselbalch equation ${ }^{132-}$ ${ }^{136}: \mathrm{pK}_{\mathrm{a}}=-\log \left(\mathrm{K}_{\mathrm{a}}\right)=-\log \left(\frac{\mathrm{c}\left(\mathrm{R}-\mathrm{COO}^{-}\right) \times \mathrm{c}\left(\mathrm{H}^{+}\right)}{\mathrm{c}(\mathrm{R}-\mathrm{COOH})}\right)=-\log \left(\frac{\mathrm{c}\left(\mathrm{R}-\mathrm{COO}^{-}\right)}{\mathrm{c}(\mathrm{R}-\mathrm{COOH})}\right)-\log \left(\mathrm{c}\left(\mathrm{H}^{+}\right)\right)=$ $-\log \left(\frac{\mathrm{c}\left(\mathrm{R}-\mathrm{COO}^{-}\right)}{\mathrm{c}(\mathrm{R}-\mathrm{COOH})}\right)+\mathrm{pH} \quad$ leading to

$$
\mathrm{pH}=\mathrm{pK}_{\mathrm{a}}+\log \frac{\mathrm{c}\left(\mathrm{R}-\mathrm{COO}^{-}\right)}{\mathrm{c}(\mathrm{R}-\mathrm{COOH})}
$$


One can thus predict the percent of ionization ( $\left.\mathrm{J}_{\mathrm{R}-\mathrm{COO}}\right)$ at certain $\mathrm{pH}$ for weak acid as follows: $\quad \mathrm{J}_{\mathrm{R}-\mathrm{COO}^{-}}=\frac{\mathrm{c}\left(\mathrm{R}-\mathrm{COO}^{-}\right)}{\mathrm{c}(\mathrm{R}-\mathrm{COOH})+\mathrm{c}\left(\mathrm{R}-\mathrm{COO}^{-}\right)}=\frac{1}{\frac{\mathrm{c}(\mathrm{R}-\mathrm{COOH})}{\mathrm{c}\left(\mathrm{R}-\mathrm{COO}^{-}\right)}+1}=\frac{1}{1+\left(\frac{\mathrm{c}\left(\mathrm{R}-\mathrm{COOO}^{-}\right)}{\mathrm{c}(\mathrm{R}-\mathrm{COOH})}\right)^{-1}}=$ $\frac{1}{1+\left(10^{\left.\mathrm{pH}-\mathrm{pK}_{\mathrm{a}}\right)^{-1}}\right.}$, leading to

$$
\mathrm{J}_{\mathrm{R}-\mathrm{COO}^{-}}=\frac{100 \%}{1+10^{\mathrm{pK}} \mathrm{a}-\mathrm{pH}}
$$

Thus, by increasing the NP suspension's $\mathrm{pH}$, the denominator in Equation X.2.5 decreases and thus the percent ionized as wells as the anionic surface charge density of the NP increases. Instead, by decreasing the NP suspension's $\mathrm{pH}$, the denominator in Equation X.2.5 increases and thus the anionic surface charge density of the NPS decreases. In the latter case, vanishing electrostatic repulsion between NPs induces NP aggregation and increases their effective hydrodynamic diameter at high $\mathrm{pH}$ values ( $c f$. $\S X .4)$.

For NPs with ligands containing weak basic functional groups (e.g. $\mathrm{R}^{-\mathrm{NH}_{3}}$ ), the NPs' effective surface charge increases at low $\mathrm{pH}$ (i.e. high concentration of $\mathrm{H}^{+}$) due to protonation of the amine groups:

$$
\mathrm{R}-\mathrm{NH}_{4}^{+} \leftrightarrow \mathrm{R}-\mathrm{NH}_{3}+\mathrm{H}^{+}
$$

This equilibrium is described by the $\mathrm{pK}_{\mathrm{a}}$ value.

$$
\begin{aligned}
& \mathrm{K}_{\mathrm{a}}=\frac{\mathrm{c}\left(\mathrm{R}-\mathrm{NH}_{3}\right) \times \mathrm{c}\left(\mathrm{H}^{+}\right)}{\mathrm{c}\left(\mathrm{R}-\mathrm{NH}_{4}^{+}\right)} \\
& \mathrm{pK}_{\mathrm{a}}=-\log \left(\mathrm{K}_{\mathrm{a}}\right)
\end{aligned}
$$

This leads to the Henderson-Hasselbalch equation:

$$
\mathrm{pH}=\mathrm{pK}_{\mathrm{a}}+\log \frac{\mathrm{c}\left(\mathrm{R}-\mathrm{NH}_{3}\right)}{\mathrm{c}\left(\mathrm{R}-\mathrm{NH}_{4}{ }^{+}\right)}
$$

Again, one can predict the percentage of unionization $\left(\mathrm{J}_{\mathrm{R}-\mathrm{NH} 3}\right)$ at certain $\mathrm{pH}$ for weak base as follows:

$$
\mathrm{J}_{\mathrm{R}-\mathrm{NH}_{3}}=\frac{\mathrm{c}\left(\mathrm{R}-\mathrm{NH}_{3}\right)}{\mathrm{c}\left(\mathrm{R}-\mathrm{NH}_{4}{ }^{+}\right)+\mathrm{c}\left(\mathrm{R}-\mathrm{NH}_{3}\right)}=\frac{100 \%}{1+10^{0 \mathrm{~K}_{\mathrm{a}}-\mathrm{pH}}}
$$

Thus, by lowering the $\mathrm{pH}$ of suspension containing NPs with basic functional group, the denominator in Equation X.2.10 increases and thus the percent unionized amines 
decreases (i.e. the ionization and cationic surface charge density of NP increases). Instead, increasing the NP suspension $\mathrm{pH}$, the denominator in Equation X.2.10 decreases and thus the percent unionized increases (ionization and cationic surface charge density of NP decreases). In the latter case, vanishing electrostatic repulsion between NPs induces NP aggregation and increases their effective hydrodynamic diameter at high $\mathrm{pH}$ values ( $c f$. $\S$ X.4). There are also charged groups whose charge is independent from the $\mathrm{pH}$. For ammonium salts such as $\mathrm{N}, \mathrm{N}, \mathrm{N}$-trimethylammonium-2ethyl methacrylate iodide there is full dissociation, leading to permanent positive surface charges ${ }^{87,88}$.

In Figure X.2.4 the dependence of the hydrodynamic diameter of different negatively charged NPs on the $\mathrm{pH}$ of the solution is shown. For this the hydrodynamic diameter $\mathrm{d}_{\mathrm{h}}$ of NPs was measured with DLS during a titration with $\mathrm{HCl}$ (aq.). The NPs were suspended in $1.5 \mathrm{mM} \mathrm{NaOH}$ (aq.), setting a total volume of $10 \mathrm{~mL}$ and concentrations of $\mathrm{C}_{\mathrm{NP}}=8.3$ or $25 \mathrm{nM}$. The titrant was aqueous $\mathrm{HCl}$ with a concentration of $12 \mathrm{mM}$. $\mathrm{HCl}$ was added using a Malvern MPT-2 autotitrator and the hydrodynamic diameter was measured with a Malvern Zetasizer nano ZS DLS set-up. 

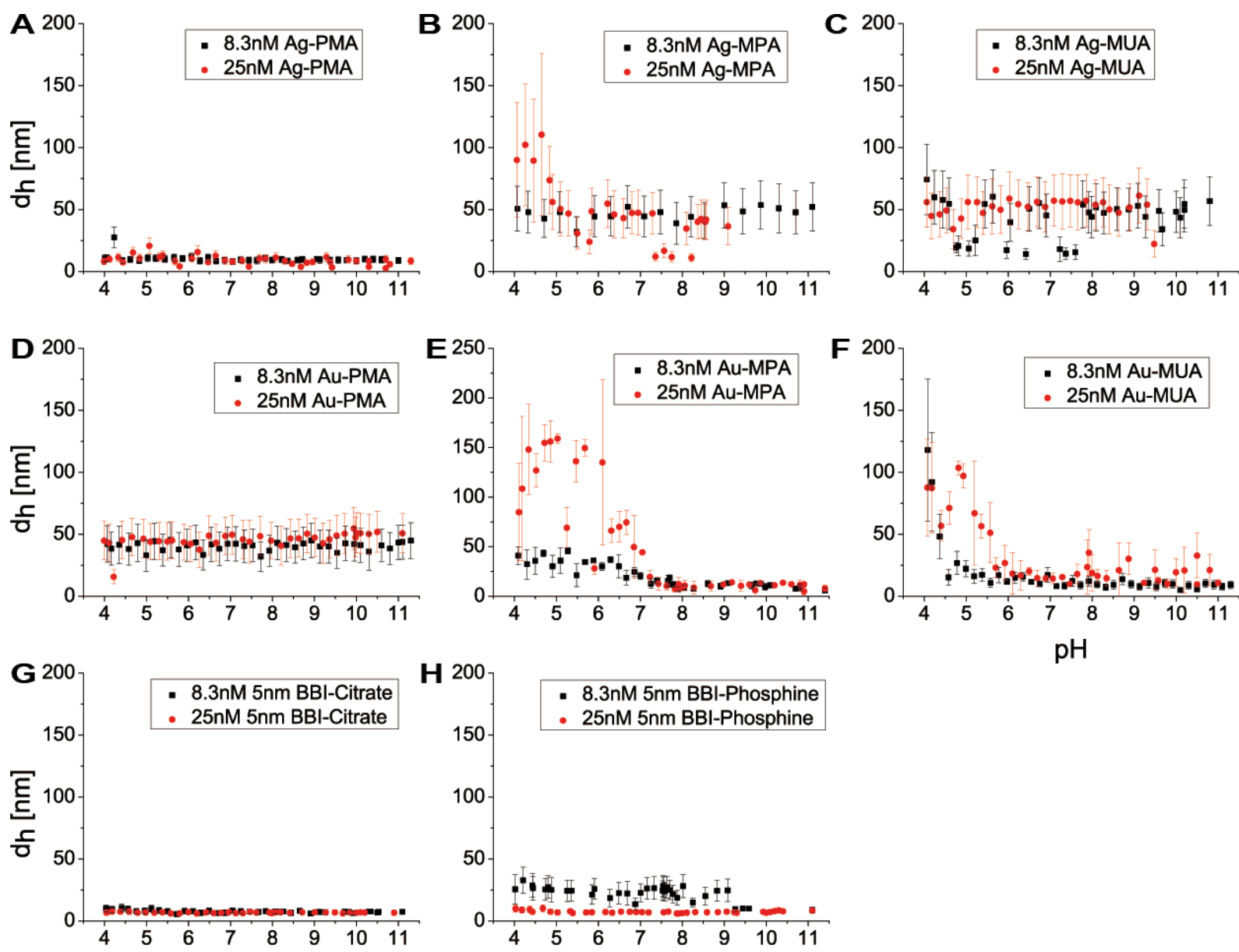

$\mathrm{pH}$
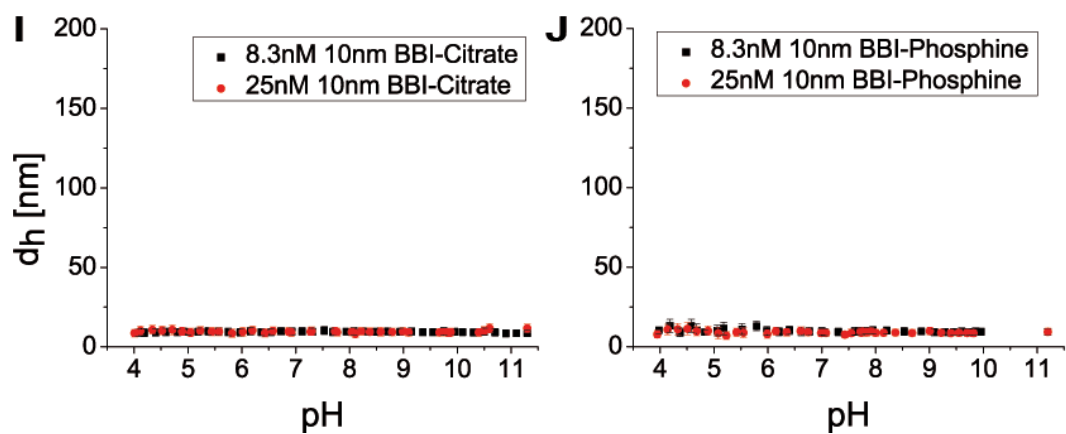

Figure X.1.4. Titration of $\mathrm{Ag}$ and $\mathrm{Au} N \mathrm{~N}$ s suspended in $1.5 \mathrm{mM} \mathrm{NaOH}$ (aq.) with a concentration of $c_{N P}=8.6 \mathrm{nM}(\cdot)$ and $c_{N P}=25 \mathrm{nM}(\bullet)$, with a total volume of $10 \mathrm{~mL} . \mathrm{HCl}$ (aq.) with a concentration of $12 \mathrm{mM}$ was chosen as titrant and was added using a Malvern MPT-2 autotitrator within a $\mathrm{pH}$ range from 11 to 4 . The hydrodynamic radius $d_{h}$ is plotted against the $\mathrm{pH}$ to detect size-dependent changes and aggregation of the NPs. $\approx 4 \mathrm{~nm}$ core diameter Ag NPs (cf. §I.3) A) overcoated with PMA (cf. §VI.3), B) after ligand exchange with MPA (cf. §V.2), C) after ligand exchange with MUA (cf. §V.2). $\approx 4$ $n m$ core diameter Au NPs (cf. §I.2) D) overcoated with PMA (cf. §VI.3), E)) after ligand exchange with MPA (cf. §V.2). F) after ligand exchange with MUA (cf. §V.2). $\approx 5 \mathrm{~nm}$ core diameter $G$ ) Cit-Au NPs (purchased from BBI) and $H$ ) after ligand exchange to bis(p-sulfonatophenyl)-phenylphosphine (cf. §III.2). $\approx 10 \mathrm{~nm}$ core diameter I) Cit-Au NPs 
(purchased from BBI) and J) after ligand exchange to bis(p-sulfonatophenyl)phenylphosphine (cf. §III.2).

\section{X.3 Laser Doppler anemometry}

As pointed out in $\S X .1$ and $\S X .2$ the surface charge of colloidal NPs provides colloidal stability due to electrostatic repulsion forces. Effective surface charge depends on many variables such as solvent type and its $\mathrm{pH}$. In case of NPs with known surface chemistry and geometry, titration curves ( $c f . \S X .4$ ) can be used to estimate the number of charged groups on the surface of a single NP. Alternatively, and most commonly used, the so-called zeta-potential $\zeta^{130,131,137}$ of the NPs can be measured. The zetapotential describes the electric potential of the NPs at the distance of the electrostatic screening length (which depends on the ionic strength of the aqueous solution). While in principle electric potential and electric charge are coupled via the Poisson-Boltzman equation, the complicated geometry of NPs with inorganic core, organic shell, and adsorbed counter ions, in general does not allow for deriving the surface charge from the zeta-potential. However, the sign of the surface charge can be concluded from the sign of the measured zeta-potential. In addition, the magnitude of the zeta-potential provides a good indicator of the NPs' colloidal stability. Generally, zeta-potential values $|\zeta|>20-30 \mathrm{mV}$ are required to maintain colloidal stability and prevent NP aggregation.

One way to measure zeta-potentials is laser Doppler anemometry (LDA). An electric field is applied and the velocity of charged NPs in this field is detected via the Doppler effect. From this the electrophoretic mobility, defined as the quotient of the drift velocity and the applied electric field, can be calculated. By using different models

from electrophoretic mobility the zeta-potential can be estimated ${ }^{131}$. Often DLS and LDA are combined in the same set-up, such as in the Zetasizer from Malvern. Note, that for LDA special cuvettes are required, which comprise electrodes to apply the electric field. Note also, that during measurements NP suspensions must cover these electrodes, and thus before measurements it has to be made sure that electrodes are functional, and if required the cuvette needs to be changed. In Figure X.3.1 the distribution of the zeta-potential is shown for PMA-Au NPs. The zeta-potential is then taken as the mean value from the distribution function. 


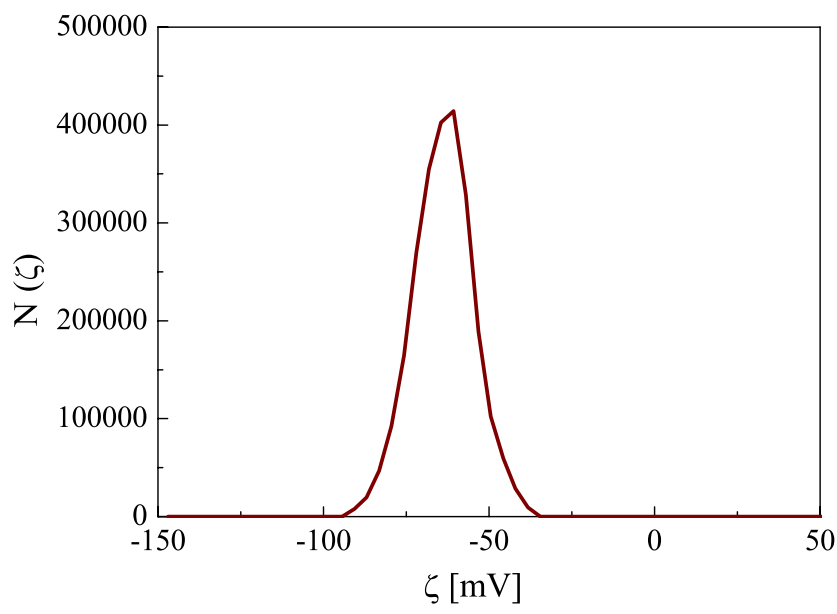

Figure X.3.1. Distribution $N(\zeta)$ of the zeta-potential $\zeta$ as measured in water from a sample of $\approx 4 \mathrm{~nm}$ core diameter PMA-Au NPs with $c_{N P}=10 \mathrm{nM}$. The obtained mean value for the zeta potential is $-48.07 \pm 1.16 \mathrm{mV}$.

In Figure X.3.2 results for zeta-potential measurements for $\mathrm{Au}$ and Ag NPs with different surface coating at different $\mathrm{pH}$ values are shown. Due to the coupled system of an auto-titrator and a Zetasizer, it was possible to use the same cuvette for $d_{h}$ (DLS, $c f . \S \times .2$ ) and zeta potential (LDA) measurements before adding more acid and pursue the titration (i.e. lowering of the $\mathrm{pH}$ ). Thus, the NP concentrations and volumes of added acid are the same as shown in $\S$ X.2, as DLS and LDA measurements were performed simultaneously. The data indicate, that according to Equation X.2.1 at low $\mathrm{pH}$ values the negatively charged $-\mathrm{COO}^{-}$groups get protonated, the zeta-potential thus becomes less negative, and thus the colloidal stability of the NPs is decreased. 

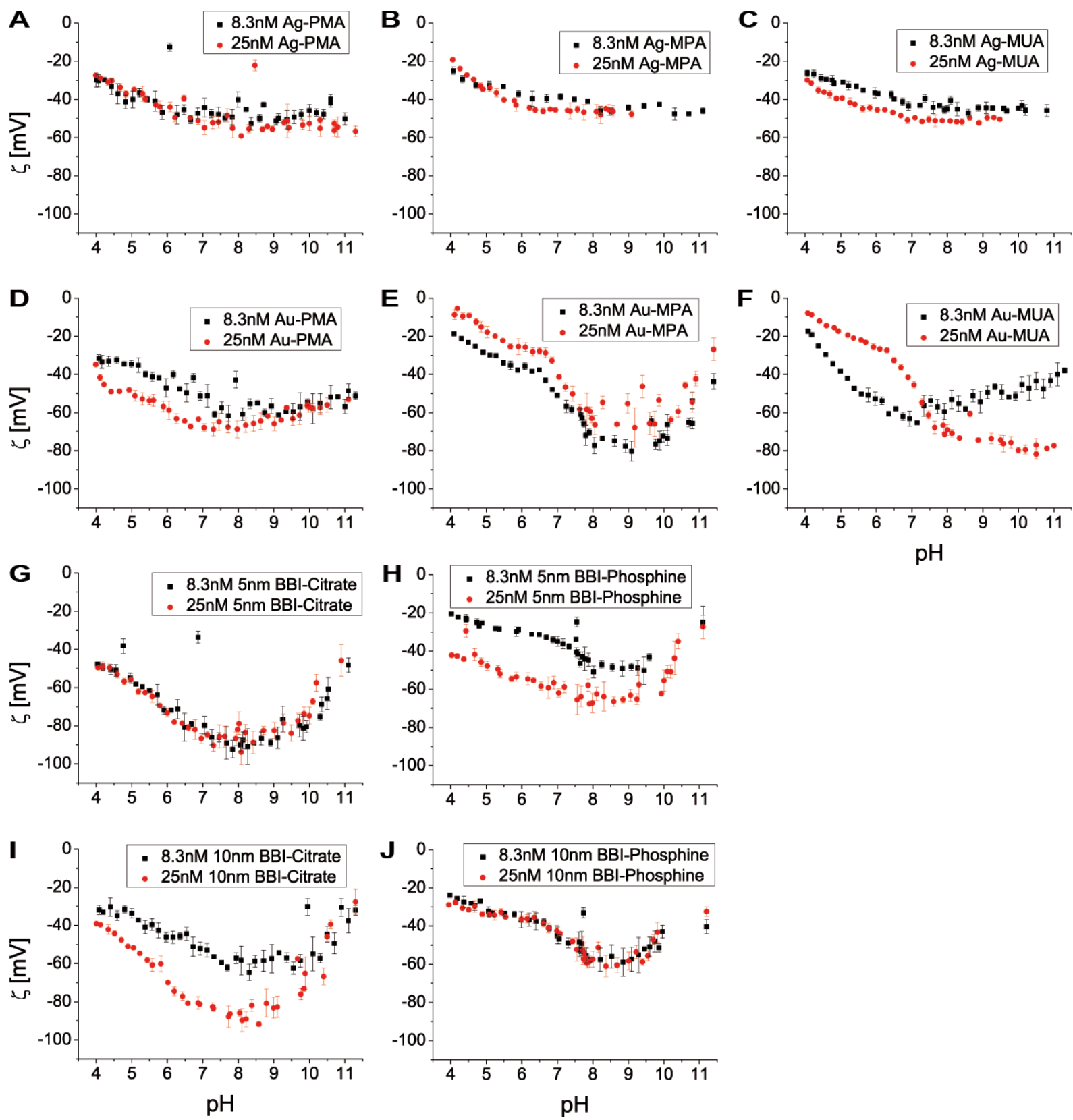

Figure X.3.2. Titration of $\mathrm{Ag}$ and $\mathrm{Au}$ NPs suspended in $1.5 \mathrm{mM} \mathrm{NaOH}$ (aq.) with a concentration of $c_{N P}=8.6 \mathrm{nM}(\bullet)$ and $c_{N P}=25 \mathrm{nM}(\bullet)$, with a total volume of $10 \mathrm{~mL}$. $\mathrm{HCl}$ (aq.) with a concentration of $12 \mathrm{mM}$ was chosen as titrand and was added using a Malvern MPT-2 autotitrator within a $\mathrm{pH}$ range from 11 to 4 . The zeta-potential $\zeta$ is plotted against the $\mathrm{pH}$. These measurements were done simultaneously to the DLS measurements shown in Figure X.2.4. $\approx 4 \mathrm{~nm}$ core diameter Ag NPs (cf. §l.3) A) overcoated with PMA (cf. §VI.3), B) after ligand exchange with MPA (cf. §V.2), C) after ligand exchange with MUA (cf. §V.2). $\approx 4 \mathrm{~nm}$ core diameter Au NPs (cf. §l.2) D) overcoated with PMA (cf. §VI.3), E) after ligand exchange with MPA (cf. §V.2), F) after ligand exchange with MUA (cf. $\S V .2)$. $\approx 5 \mathrm{~nm}$ core diameter $G$ ) Cit-Au NPs (purchased from $B B I$ ) and $H$ ) after ligand exchange to bis(p-sulfonatophenyl)-phenylphosphine (cf. 
\$III.2). $\approx 10 \mathrm{~nm}$ core diameter I) Cit-Au NPs (purchased from BBI) and J) after ligand exchange to bis(p-sulfonatophenyl)-phenylphosphine (cf. §III.2).

\section{X.4 pH titration}

Acid-base titration is a well-established chemical method in which $\mathrm{pH}$ is measured as function of the amount of added acid or base. The resulting plot is called titration curve, which can be used to determine the $\mathrm{pK}_{\mathrm{a}}$ of the NPs and more importantly, the surface density of acidic or basic ligands. Here, the principle is demonstrated for NPs with $-\mathrm{COOH}$ groups present on their surface. In order to determine titration curves over a large spectrum and to guarantee fully deprotonated carboxylic moieties, the NPs were first diluted in $\mathrm{NaOH}$ (aq.) $(\mathrm{pH}=11)$. The basic NP suspension was then titrated using $\mathrm{HCl}$ (aq.) (cf. Figure X.4.1). First, added $\mathrm{H}^{+}$(from $\mathrm{HCl}$ ) can react with free $\mathrm{OH}^{-}$(from $\mathrm{NaOH}$ ) in suspension. This leads to the first plateau followed by the first steep drop and equivalent point (ep $\mathrm{e}_{1}$ in Figure X.4.1). Upon consumption of all free $\mathrm{OH}^{-}$ ions, the carboxylates present on the NP surface become protonated, resulting in the second plateau (buffer plateau). Further addition of $\mathrm{HCl}$ leads to the second steep drop and equivalent point $\left(\mathrm{ep}_{2}\right.$ in Figure X.4.1). Further addition of $\mathrm{HCl}$ leads to the third plateau at low $\mathrm{pH}$. To determine the $\mathrm{pK}_{\mathrm{a}}$ the $\mathrm{pH}$ value of the halfway point of the second plateau needs to be considered ( 7 in this case). In this experiment, NPs were suspended in aqueous $\mathrm{NaOH}$ solution $\left(1.5 \mathrm{mM}, \mathrm{V}_{\mathrm{NaOH} \_ \text {sol }}=10 \mathrm{~mL}\right.$ ) to a concentration of $\mathrm{C}_{\mathrm{NP}}=8.6 \mathrm{nM}$. HCl solution $(2.5 \mathrm{mM})$ was added in $100 \mu \mathrm{L}$ steps to the vigorously stirring NP suspension using the Malvern MPT-2 autotitrator. The resulting volume of the solution is $\mathrm{V}_{\text {sol }}=\mathrm{V}_{\mathrm{NaOH} \_ \text {sol }}+\mathrm{V}_{\mathrm{HC} \text { _sol }}$. After the addition of each volume of acid the solution was given $5 \mathrm{~s}$ to equilibrate and then the $\mathrm{pH}$ was measured using a Sartorius (PP 50) $\mathrm{pH}$ meter. The experiments were repeated after $24 \mathrm{~h}$ to confirm the data and ensure reproducibility. The $\mathrm{pK}_{\mathrm{a}}$ value of the NPs was found to be around $\approx 7$ as per the titration curve in Figure X.4.1. By adding more acid the charged groups became saturated with protons, until the $\mathrm{pH}$ led to aggregation of the NPs at a $\mathrm{pH}$ of around 45 due to the loss of surface charge. Examples of titration curves with determined $\mathrm{pK}_{\mathrm{a}}$ values for various NPs with different surface chemistries are shown in Figure X.4.2. 


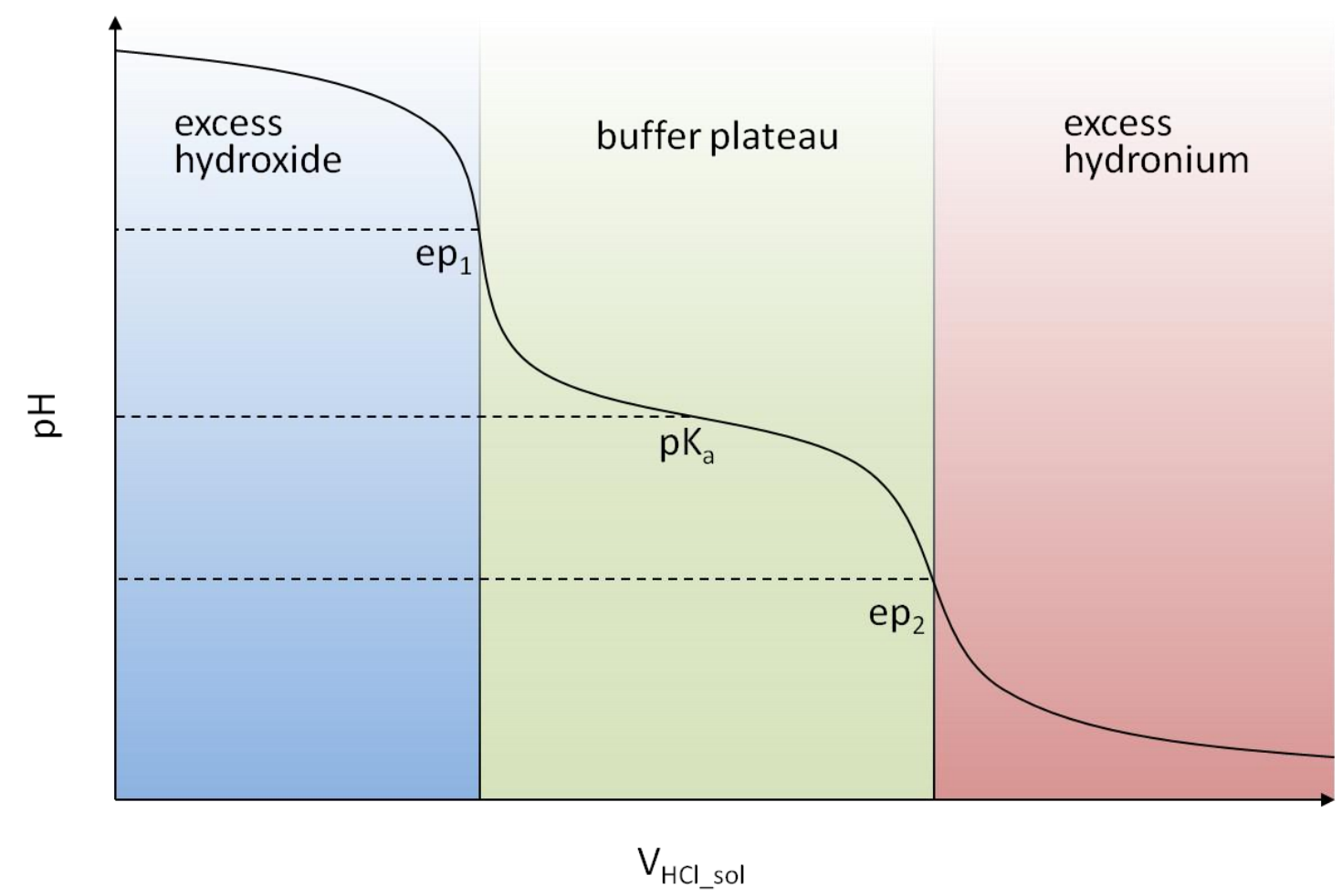

Figure X.4.1. Illustrative titration curve of carboxylate stabilized NPs suspended in $\mathrm{NaOH}$ (aq.) with the original volume $\mathrm{V}_{\mathrm{NaOH} \text { _sol }}$ against $\mathrm{HCl}$ (aq.), of which the volume $V_{H C l}$ sol $h a s$ been added. The figure is adopted and modified from Charron et al ${ }^{60}$. 

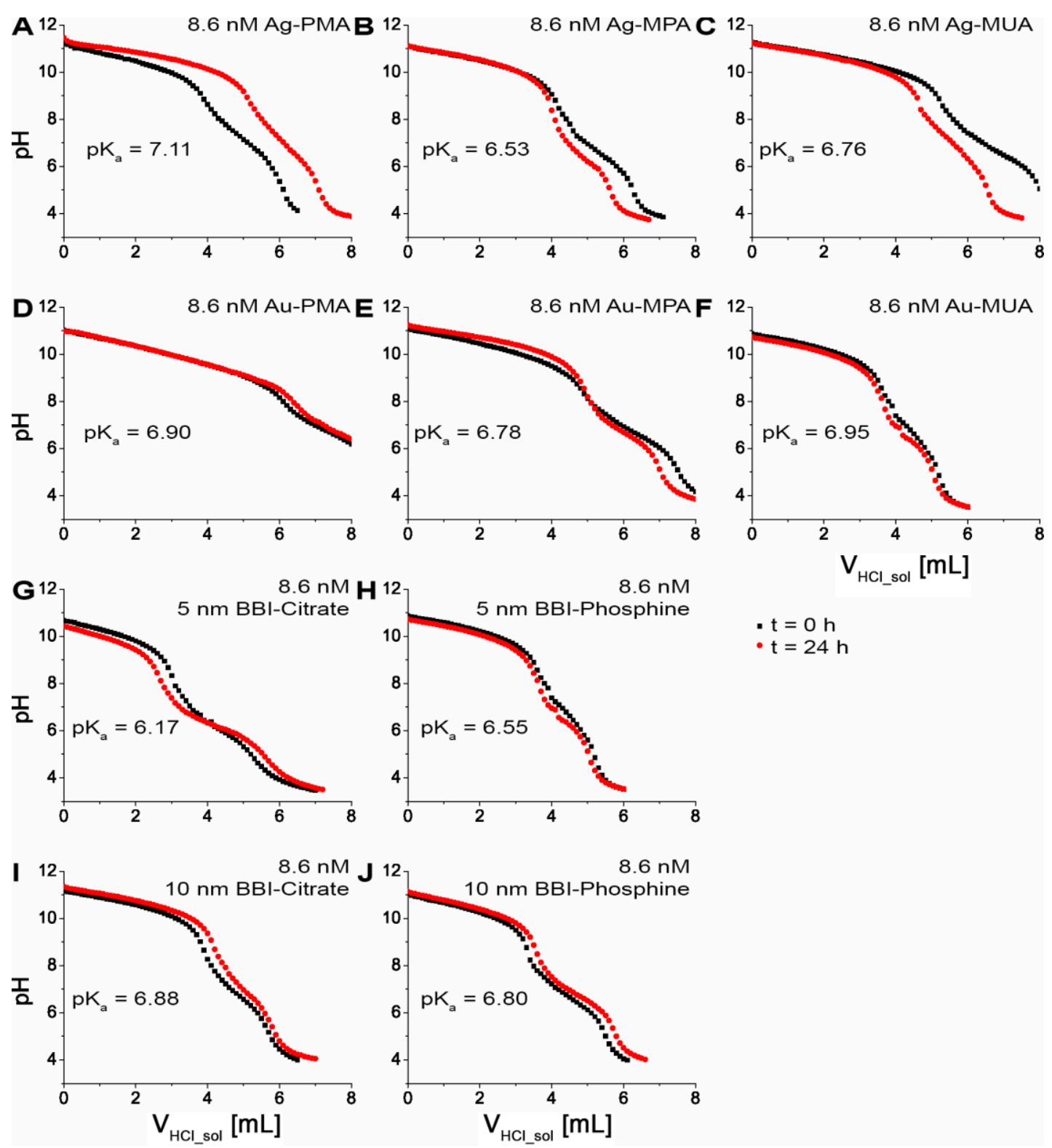

Figure X.4.2. $p H$ titration of $A g$ and $A u$ NPs with different surface chemistry originally suspended in $1.5 \mathrm{mM} \mathrm{NaOH}$ (aq.) with a concentration of $c_{N P}=8.6 \mathrm{nM}$ and a total volume of $\mathrm{V}_{\mathrm{NaOH} \text { sol }}=10 \mathrm{~mL}$. $\mathrm{HCl}$ (aq.) with a concentration of $2.5 \mathrm{mM}$ was chosen as titrand and was added in steps of $\Delta V_{\text {HCIsol }}=100 \mu \mathrm{L}$ to the NP suspension under vigorous stirring. After the first titration at $t=0 \mathrm{~h}(\mathbf{0})$ the experiments were repeated at $t=24 h(\bullet)$. The NP samples are the same as used in Figure X.2.4 and Figure X.3.2: $\approx 4$ $n m$ core diameter Ag NPs (cf. §l.3) A) overcoated with PMA (cf. §VI.3), B) after ligand exchange with MPA (cf. $\S V .2), C)$ after ligand exchange with MUA (cf. $\S V .2) . \approx 4 \mathrm{~nm}$ core diameter Au NPs (cf. §I.2) D) overcoated with PMA (cf. §VI.3), E) after ligand exchange with MPA (cf. $\S V .2), F)$ after ligand exchange with MUA (cf. $\S V .2)$ ) $\approx 5 \mathrm{~nm}$ core diameter Au NPS G) citrate coated (purchased from BBI) and H) after ligand exchange to bis(p-sulfonatophenyl)-phenylphosphine (cf. §III.2). $\approx 10 \mathrm{~nm}$ core diameter 
Au NPs I) citrate coated (purchased from BBI) and J) after ligand exchange to bis( $p$ sulfonatophenyl)-phenylphosphine (cf. §III.2).

If the concentration of NPs ( $\mathrm{c}_{\mathrm{NP}}$ ) is known, the number of carboxyl groups on the surface of a single NP can be estimated as described by Charron et $a l^{60}$. For example, from Figure X.4.2, the volume of $\mathrm{HCl}\left(\Delta \mathrm{V}_{\mathrm{HCl} \_ \text {sol }}\right)$ used to protonate $\mathrm{R}-\mathrm{COO}^{-}$on the surface of the NPs can be determined as the length of the buffer plateau.

$$
\Delta \mathrm{V}_{\text {HCI_sol }}=\mathrm{V}_{\text {HCI_sol }}\left(\mathrm{ep}_{2}\right)-\mathrm{V}_{\text {HCI_sol }}\left(\mathrm{ep}_{1}\right)
$$

Since the concentration of $\mathrm{HCl}\left(\mathrm{C}_{\mathrm{HCl}}\right)$ and the volume of initial NP suspension $\left(\mathrm{V}_{\mathrm{NP}_{-} \text {sol }}=\right.$ $\left.\mathrm{V}_{\mathrm{NaOH} \_s o l}\right)$ are known, the concentration of all carboxylate ions on NPs ( $\left.\mathrm{C}_{\mathrm{R}-\mathrm{coO}-}\right)$ can be calculated as follows:

$$
\mathrm{C}_{\mathrm{R}-\mathrm{COO}^{-}} \times \mathrm{V}_{\mathrm{NP}_{-} \text {sol }}=\mathrm{c}_{\mathrm{HCl}} \times \Delta \mathrm{V}_{\mathrm{HCl}_{-} \text {sol }}
$$

(Equation X.4.2)

The number of acid groups per NP ( $\left.\mathrm{N}_{\mathrm{R}-\mathrm{COO}-/ \mathrm{NP}}\right)$ thus should be:

$$
\mathrm{N}_{\mathrm{R}-\mathrm{COO}^{-} / \mathrm{NP}}=\frac{\mathrm{c}_{\mathrm{R}-\mathrm{COO}^{-}}}{\mathrm{c}_{\mathrm{NP}}}
$$

In case of PMA-coated NPS, $\mathrm{N}_{\mathrm{R}-\mathrm{COO}-\mathrm{NP}}$ can be compared with $\mathrm{N}_{\mathrm{P} / \mathrm{NP}}$ as described in $\S$ VIII.6. Each polymer monomer unit in fully deprotonated state bears one or two negative charges, in case the PMA backbone is linked to a side chain or hydrolyses, respectively ${ }^{87}$.

$$
\mathrm{N}_{\mathrm{R}-\mathrm{COO}-\mathrm{NP}}=\left(2-\mathrm{R}_{\text {chain } / \text { mon }}\right) \times \mathrm{N}_{\mathrm{P} / \mathrm{NP}}
$$

While in Figure $\mathrm{X} .4 .2 \mathrm{D}$ the second equivalence point is not in the measured $\mathrm{pH}$ range, roughly $\Delta \mathrm{V}_{\mathrm{HCl} \_ \text {sol }} \approx 2 \mathrm{~mL}$. With $\mathrm{C}_{\mathrm{HCl}}=2.5 \mathrm{mM}, \mathrm{C}_{\mathrm{NP}}=8.6 \mathrm{nM}, \mathrm{V}_{\mathrm{NP}_{-} \text {sol }}=\mathrm{V}_{\mathrm{NaOH}_{-} \text {sol }}=10 \mathrm{~mL}$ (cf. Figure $\mathrm{X} .4 .2)$ this leads to $\mathrm{N}_{\mathrm{R}-\mathrm{COO}-/ \mathrm{NP}}=\left(\left(\mathrm{C}_{\mathrm{HCl}} \times \Delta \mathrm{V}_{\mathrm{HCl} \_ \text {sol }}\right) / \mathrm{V}_{\mathrm{NP} \_ \text {sol }}\right) / \mathrm{c}_{\mathrm{NP}}=\left(\left(2.5 \times 10^{-3} \mathrm{M} \times 2\right.\right.$ $\mathrm{mL}) / 10 \mathrm{~mL}$ )/ $8.6 \times 10^{-9} \mathrm{M} \approx 58,000$. In $\S \mathrm{VIII.6}$ TGA analysis of similar PMA-Au NPs (ca. 4 $\mathrm{nm}$ core diameter) yielded $\mathrm{N}_{\mathrm{P} / \mathrm{NP}}=270$. Thus, with $\mathrm{R}_{\text {chain/mon }}=0.75$ this leads to $\mathrm{N}_{\mathrm{R} \text {-coo- }}$ $/ N P=\left(2-R_{\text {chain } / m o n}\right) \times N_{P / N P}=(2-0.75) \times 270 \approx 338$. The discrepancy in values can be explained by the different degree of purification. While the PMA-Au NPs in case of TGA analysis had been purified by ultracentrifugation $\left(\mathrm{N}_{\mathrm{R}-\mathrm{COO}-/ \mathrm{NP}}=338\right)$, the PMA-Au NPs in case of titration analysis $\left(\mathrm{N}_{\mathrm{R}-\mathrm{COO}-\mathrm{NP}}=58,000\right)$ had been purified by gel electrophoresis only, which may result in some remaining polymer micelles, which contribute to the amount of $\mathrm{COO}^{-}$groups. These number examples point out again the necessity of applying different characterization techniques. 


\section{Conclusions}

Synthetic protocols in this contribution described the preparation of hydrophobic NPs of different types ( $\mathrm{Au}, \mathrm{Ag}$, $\mathrm{FePt}, \mathrm{Fe}_{3} \mathrm{O}_{4}, \mathrm{Sn}, \mathrm{CdSe}$, and $\mathrm{CdSe} / \mathrm{ZnS}$ ). Moreover, preparation of hydrophilic Au and Ag NPs of various shapes (spheres, rods, stars) and sizes $(5-100 \mathrm{~nm})$ are detailed. The prepared NPs showed good monodispersity as evident from corresponding TEM analysis. Surface functionalization strategies including ligand exchange and polymer overcoating were employed on selected NPs to control their hydrophobicity, hydrophilicity, and colloidal stability. For example, ligand exchange on citrated-capped Au NPs with alkylamine induced phase transfer from water to chloroform. On the other hand, ligand exchange on alkanethiol-capped gold NPs with 3-mercaptopropionic acid molecules facilitated the phase transfer of these NPs into aqueous phase from chloroform. Similarly, carefully designed amphiphilic polymer was capable to overcoat alkanethiol-capped Au NPs and render their surface hydrophilic allowing facile transfer into aqueous phase from chloroform.

Purification of one type of NPs (namely PMA-g-dodecyl-capped Au NPs $\approx 4 \mathrm{~nm}$ diameter, $c f$. $§ I .2, \S V I .3)$ was evaluated using various methods: syringe filtration, ultracentrifugation, size exclusion chromatography, and gel electrophoresis. These results showed different purity levels for different purification methods (for example ultracentrifugation resulted in less free polymer micelles as compared to gel electrophoresis), highlighting the importance of optimizing the purification process for a specific synthesis.

Various characterization techniques confirmed the ability to probe the structural, photophysical, and colloidal properties of NPs. For example, visualization of PEGcapped Au NPs with TEM coupled to negative staining confirmed the NPs' core dimension and, more importantly, a well-defined polymeric shell surrounding each NP. Thermogravimetric analysis of dodecanethiol-capped Au NPs confirmed the presence of the organic shell on NPs and allowed for quantifying its weight percentage per NP. FTIR and NMR analysis confirmed overcoating of dodecanethiol-capped Au NPs with PMA polymer, highlighting the importance of these analytical techniques to follow surface functionalization of NPs. UV/vis absorption was employed successfully to quantify the concentration of Au NPs, to follow their salt- or $\mathrm{pH}$-induced aggregation by monitoring the plasmon peak shift and broadening, and to quantify the loaded dye per single NP. Finally, effective surface charge analysis as measured by laser Doppler anemometry upon acid-base titration allowed for the determination of the acidity/basicity, $\mathrm{pK}_{\mathrm{a}}$, and the number of acidic or basic groups on a single PMA-gdodecyl-capped Au NP. 
On purpose discrepancies in quantitative results as obtained with different characterization techniques are presented. In fact, different characterization techniques analyzed different parameters, and to relate them idealized models, like the sketches in Figure VI.1.1 have to be used. Also the degree of purity plays a major role in quantitative analysis.

To the end there is no "optimum" characterization technique. In this contribution an overview was presented about the characterization techniques routinely used in one selected laboratory, and what quantitative information can be obtained from them. Collectively, the described details in this contribution should shed the light on important corners in the field of inorganic NP synthesis, characterization and surface functionalization.

\section{Acknowledgements}

This work was supported by the German Research Foundation (DFG grant PA 794/251) and by the European Commission (project FutureNanoNeeds). CCC acknowledges the Spanish Ministerio de Economía y Competitividad for a Juan de la Cierva-Incorporación contract. AE acknowledges Junta de Andalucía (Spain) for a Talentia Postdoc Fellowship, co-financed by the European Union Seventh Framework Programme, grant agreement no 267226. 


\section{References}

(1) Parak, W. J., Complex Colloidal Assembly. Science 2011, 334, 1359-1360.

(2) Frens, G., Controlled Nucleation for Regulation of Particle-Size in Monodisperse Gold Solutions. Nature Physical Science 1973, 241, (105), 20-22.

(3) Bastus, N. G.; Comenge, J.; Puntes, V., Kinetically Controlled Seeded Growth Synthesis of Citrate-Stabilized Gold Nanoparticles of up to $200 \mathrm{~nm}$ : Size Focusing versus Ostwald Ripening. Langmuir 2011, 27, (17), 11098-11105.

(4) Bastus, N. G.; Merkoci, F.; Piella, J.; Puntes, V., Synthesis of Highly Monodisperse CitrateStabilized Silver Nanoparticles of up to $200 \mathrm{~nm}$ : Kinetic Control and Catalytic Properties. Chemistry of Materials 2014, 26, (9), 2836-2846.

(5) Pellegrino, T.; Kudera, S.; Liedl, T.; Javier, A. M.; Manna, L.; Parak, W. J., On the Development of Colloidal Nanoparticles towards Multifunctional Structures and their Possible Use for Biological Applications. Small 2005, 1, (1), 48-63.

(6) Sperling, R. A.; Parak, W. J., Surface modification, functionalization and bioconjugation of colloidal inorganic nanoparticles. Philosophical Transactions of the Royal Society A Mathematical Physical and Engineering Science 2010, 368, (1915), 1333-1383.

(7) Montenegro, J.-M.; Grazu, V.; Sukhanova, A.; Agarwal, S.; Fuente, J. M. d. I.; Nabiev, I.; Greiner, A.; Parak, W. J., Controlled antibody/(bio-) conjugation of inorganic nanoparticles for targeted delivery. Advanced Drug Delivery Reviews 2013, 65, 677-688.

(8) Alkilany, A.; Murphy, C., Toxicity and cellular uptake of gold nanoparticles: what we have learned so far? Journal of Nanoparticle Research 2010, 12, (7), 2313-2333.

(9) Brust, M.; Walker, M.; Bethell, D.; Schiffrin, D. J.; Whyman, R., Synthesis of ThiolDerivatized Gold Nanoparticles in a 2-Phase Liquid-Liquid System. J. Chem. Soc., Chem. Commun. 1994, 1, (7), 801-802.

(10) Lin, C.-A. J.; Sperling, R. A.; Li, J. K.; Yang, T.-Y.; Li, P.-Y.; Zanella, M.; Chang, W. H.; Parak, W. J., Design of an Amphiphilic Polymer for Nanoparticle Coating and Functionalization. Small 2008, 4, (3), 334-341.

(11) Yin, Y.; Alivisatos, A. P., Colloidal nanocrystal synthesis and the organic-inorganic interface. Nature 2005, 437, (7059), 664.

(12) Viswanatha, R.; Santra, P. K.; Dasgupta, C.; Sarma, D. D., Growth mechanism of nanocrystals in solution: ZnO, a case study. Physical Review Letters 2007, 98, (25).

(13) Thanh, N. T. K.; Maclean, N.; Mahiddine, S., Mechanisms of Nucleation and Growth of Nanoparticles in Solution. Chemical Reviews 2014, 114, (15), 7610-7630.

(14) Pensa, E.; Cortés, E.; Corthey, G.; Carro, P.; Vericat, C.; Fonticelli, M. H.; Benítez, G.; Rubert, A. A.; Salvarezza, R. C., The Chemistry of the Sulfur-Gold Interface: In Search of a Unified Model. Accounts of Chemical Research 2012, 45, 1183-1192.

(15) Häkkinen, H., The gold-sulfur interface at the nanoscale. Nature Chemistry 2012, 4, 443455.

(16) Fink, J.; Kiely, C. J.; Bethell, D.; Schiffrin, D. J., Self-Organization of Nanosized Gold Particles. Chemistry of Materials 1998, 10, (3), 922-926.

(17) Sperling, R. A.; Pellegrino, T.; Li, J. K.; Chang, W. H.; Parak, W. J., Electrophoretic Separation of Nanoparticles with a Discrete Number of Functional Groups. Advanced Functional Materials 2006, 16, (7), 943-948.

(18) Ansar, S. M.; Mohammed, F. S.; Whitell, G. v.; Budi, M.; Powell, K. C.; Mefford, O. T.; Kitchens, C. L., Effect of Postsynthesis Purifications on Gold and Silver Nanoparticle Ligand Coverage. Journal of Physical Chemistry C 2016, 120, 6842-6850.

(19) Jana, N. R.; Peng, X., Single-Phase and Gram-Scale Routes toward Nearly Monodisperse $\mathrm{Au}$ and Other Noble Metal Nanocrystals. Journal of the American Chemical Society 2003, 125, (47), 14280-14281. 
(20) Sun, Y., Controlled synthesis of colloidal silver nanoparticles in organic solutions: empirical rules for nucleation engineering. Chemical Society Reviews 2013, 42, (7), 24972511.

(21) Mari, A.; Imperatori, P.; Marchegiani, G.; Pilloni, L.; Mezzi, A.; Kaciulis, S.; Cannas, C.; Meneghini, C.; Mobilio, S.; Suber, L., High yield synthesis of pure alkanethiolate-capped silver nanoparticles. Langmuir 2010, 26, (19), 15561-15566.

(22) Sun, S.; Murray, C. B.; Weller, D.; Folks, L.; Moser, A., Monodisperse FePt Nanoparticles and Ferromagnetic FePt Nanocrystal Superlattices. Science 2000, 287, (March 17), 19891992.

(23) Sun, S.; Zeng, H.; Robinson, D. B.; Raoux, S.; Rice, P. M.; Wang, S. X.; Li, G., Monodisperse $\mathrm{MFe}_{2} \mathrm{O}_{4}(\mathrm{M}=\mathrm{Fe}, \mathrm{Co}, \mathrm{Mn}$ ) Nanoparticles. J. Am. Chem. Soc. 2004, 126, (1), 273-279.

(24) Kravchyk, K.; Protesescu, L.; Bodnarchuk, M. I.; Krumeich, F.; Yarema, M.; Walter, M.; Guntlin, C.; Kovalenko, M. V., Monodisperse and inorganically capped Sn and Sn/SnO2 nanocrystals for high-performance Li-ion battery anodes. J Am Chem Soc 2013, 135, (11), 4199-202.

(25) Peng, Z. A.; Peng, X., Formation of High-Quality CdTe, CdSe, and CdS Nanocrystals Using CdO as Precursor. Journal of the American Chemical Society 2001, 123, (1), 183-184.

(26) Dabbousi, B. O.; Rodriguez-Viejo, J.; Mikulec, F. V.; Heine, J. R.; Mattoussi, H.; Ober, R.; Jensen, K. F.; Bawendi, M. G., (CdSe)ZnS Core-Shell Quantum Dots: Synthesis and Characterization of a Size Series of Highly Luminescent Nanocrystallites. Journal of Physical Chemistry B 1997, 101, (46), 9463-9475.

(27) Song, K.-K.; Lee, S., Highly luminescent (ZnSe)ZnS core-shell quantum dots for blue to UV emission: synthesis and characterization. Current Applied Physics 2001, 1, (2-3), 169-173.

(28) Yu, W. W.; Qu, L.; Guo, W.; Peng, X., Experimental Determination of the Extinction Coefficient of CdTe, CdSe, and CdS Nanocrystals. Chemistry of Materials 2003, 15, (14), 2854-2860.

(29) Bawendi, M. G.; Steigerwald, M. L.; Brus, L. E., The quantum mechanics of large semiconductor clusters ("quantum dots"). Annual Review of Physical Chemistry 1990, 41, 477-496.

(30) Talapin, D. V.; Rogach, A. L.; Kornowski, A.; Haase, M.; Weller, H., Highly Luminescent Monodisperse CdSe and CdSe/ZnS Nanocrystals Synthesized in a HexadecylamineTrioctylphosphine Oxide-Trioctylphospine Mixture. NanoLetters 2001, 1, (4), 207-211.

(31) Xie, R.; Kolb, U.; Li, J.; Basche, T.; Mews, A., Synthesis and Characterization of Highly Luminescent CdSe-Core CdS/Zn0.5Cd0.5S/ZnS Multishell Nanocrystals. J. Am. Chem. Soc. 2005, 127, (20), 7480-7488.

(32) Yu, W. W.; Peng, X., Formation of High-Quality CdS and Other II-VI Semiconductor Nanocrystals in Noncoordinating Solvents: Tunable Reactivity of Monomers. Angewandte Chemie International Edition 2002, 41, (13), 2368-2371.

(33) Turkevich, J.; Stevenson, P. C.; Hillier, J., A study of the nucleation and growth processes in the synthesis of colloidal golde. J. Discuss. Faraday Soc. 1951, 11, 55-75.

(34) Ye, X.; Jin, L.; Caglayan, H.; Chen, J.; Xing, G.; Zheng, C.; Doan-Nguyen, V.; Kang, Y.; Engheta, N.; Kagan, C. R.; Murray, C. B., Improved Size-Tunable Synthesis of Monodisperse Gold Nanorods through the Use of Aromatic Additives. ACS Nano 2012, 6, (3), 2804-2817.

(35) Li, N.; Zhao, P.; Astruc, D., Anisotropic Gold Nanoparticles: Synthesis, Properties, Applications, and Toxicity. ANGEWANDTE CHEMIE-INTERNATIONAL EDITION 2014, 53, (7), 1756-1789.

(36) Schulz, F.; Homolka, T.; Bastus, N. G.; Puntes, V.; Weller, H.; Vossmeyer, T., Little Adjustments Significantly Improve the Turkevich Synthesis of Gold Nanoparticles. Langmuir 2014, 30, (35), 10779-10784. 
(37) Piella, J.; Bastus, N. G.; Puntes, V., Size-Controlled Synthesis of Sub-10-nanometer Citrate-Stabilized Gold Nanoparticles and Related Optical Properties. Chemistry Of Materials 2016, 28, (4), 1066-1075.

(38) Scarabelli, L.; Sánchez-Iglesias, A.; Pérez-Juste, J.; Liz-Marzán, L. M., A “Tips and Tricks" Practical Guide to the Synthesis of Gold Nanorods. The Journal of Physical Chemistry Letters 2015, 6, (21), 4270-4279.

(39) ENUSTUN, B.; TURKEVICH, J., COAGULATION OF COLLOIDAL GOLD. JOURNAL OF THE AMERICAN CHEMICAL SOCIETY 1963, 85, (21), 3317-3328.

(40) Zeng, J.; Zheng, Y. Q.; Rycenga, M.; Tao, J.; Li, Z. Y.; Zhang, Q. A.; Zhu, Y. M.; Xia, Y. N., Controlling the Shapes of Silver Nanocrystals with Different Capping Agents. Journal of the American Chemical Society 2010, 132, (25), 8552-+.

(41) Li, H. S.; Xia, H. B.; Wang, D. Y.; Tao, X. T., Simple Synthesis of Monodisperse, Quasispherical, Citrate-Stabilized Silver Nanocrystals in Water. Langmuir 2013, 29, (16), 50745079.

(42) Sondi, I.; Goia, D. V.; Matijevic, E., Preparation of highly concentrated stable dispersions of uniform silver nanoparticles. Journal of Colloid and Interface Science 2003, 260, (1), 75-81.

(43) Banerjee, S.; Loza, K.; Meyer-Zaika, W.; Prymak, O.; Epple, M., Structural Evolution of Silver Nanoparticles during Wet-Chemical Synthesis. Chemistry Of Materials 2014, 26, (2), 951-957.

(44) Ye, X.; Zheng, C.; Chen, J.; Gao, Y.; Murray, C. B., Using Binary Surfactant Mixtures To Simultaneously Improve the Dimensional Tunability and Monodispersity in the Seeded Growth of Gold Nanorods. Nano Letters 2013, 13, (2), 765-771.

(45) Jana, N. R.; Gearheart, L.; Murphy, C. J., Wet Chemical Synthesis of High Aspect Ratio Cylindrical Gold Nanorods. Journal of Physical Chemistry B 2001, 105, (18), 4065-4067.

(46) Nikoobakht, B.; El-Sayed, M. A., Preparation and Growth Mechanism of Gold Nanorods (NRs) Using Seed-Mediated Growth Method. Chem. Mater. 2003, 15, (10), 1957-1962.

(47) Murphy, C. J.; Thompson, L. B.; Chernak, D. J.; Yang, J. A.; Sivapalan, S. T.; Boulos, S. P.; Huang, J.; Alkilany, A. M.; Sisco, P. N., Gold nanorod crystal growth: From seed-mediated synthesis to nanoscale sculpting. Current Opinion In Colloid \& Interface Science 2011, 16, (2), 128-134.

(48) Alkilany, A. M.; Nagaria, P. K.; Hexel, C. R.; Shaw, T. J.; Murphy, C. J.; Wyatt, M. D., Cellular uptake and cytotoxicity of gold nanorods: molecular origin of cytotoxicity and surface effects. Small 2009, 5, (6), 701-8.

(49) Yuan, H.; Fales, A.; Vo-Dinh, T., TAT Peptide-Functionalized Gold Nanostars: Enhanced Intracellular Delivery and Efficient NIR Photothermal Therapy Using Ultralow Irradiance. JOURNAL OF THE AMERICAN CHEMICAL SOCIETY 2012, 134, (28), 11358-11361.

(50) Indrasekara, A.; Wadams, R.; Fabris, L., Ligand Exchange on Gold Nanorods: Going Back to the Future. PARTICLE \& PARTICLE SYSTEMS CHARACTERIZATION 2014, 31, (8), 819838.

(51) Pellegrino, T.; Sperling, R. A.; Alivisatos, A. P.; Parak, W. J., Gelelectrophoresis of GoldDNA Nanoconjugates. J. Biomed. Biotechnol. 2007, Article ID 26796, 1-9.

(52) Parak, W. J.; Pellegrino, T.; Micheel, C. M.; Gerion, D.; Williams, S. C.; Alivisatos, A. P., Conformation of oligonucleotides attached to gold nanocrystals probed by gel electrophoresis. Nanoletters 2003, 3, (1), 33-36.

(53) Lipka, M.; Semmler-Behnke, M.; Sperling, R. A.; Wenk, A.; Takenaka, S.; Schleh, C.; Kissel, T.; Parak, W. J.; Kreyling, W. G., Biodistribution of PEG-modified gold nanoparticles following intratracheal instillation and intravenous injection. Biomaterials 2010, 31, (25), 6574-6581. 
(54) Brandenberger, C.; Mühlfeld, C.; Ali, Z.; Lenz, A.-G.; Schmid, O.; Parak, W. J.; Gehr, P.; Rothen-Rutishauser, B., Quantitative Evaluation of Cellular Uptake and Trafficking of Plain and Polyethylene Glycol-Coated Gold Nanoparticles. Small 2010, 6, 1669-1678.

(55) del_Pino, P.; Yang, F.; Pelaz, B.; Zhang, Q.; Kantner, K.; Hartmann, R.; Baroja, N. M. d.; Gallego, M.; Möller, M.; Manshian, B. B.; Soenen, S. J.; Riedel, R.; Hampp, N.; Parak, W. J., Basic Physicochemical Properties of Polyethylene Glycol Coated Gold Nanoparticles that Determine Their Interaction with Cells. Angew Chem Int Ed Engl 2016, 55, 54835487.

(56) Soliman, M. G.; B, B. P.; Parak, W. J.; Pino, P. d., Phase transfer and polymer coating methods toward improving the stability of metallic nanoparticles for biological applications. Chemistry of Materials 2015, 27, 990-997.

(57) Lista, M.; Liu, D. Z.; Mulvaney, P., Phase Transfer of Noble Metal Nanoparticles to Organic Solvents. Langmuir 2014, 30, (8), 1932-1938.

(58) Serrano-Montes, A. B.; de Aberasturi, D. J.; Langer, J.; Giner-Casares, J. J.; Scarabelli, L.; Herrero, A.; Liz-Marzan, L. M., A General Method for Solvent Exchange of Plasmonic Nanoparticles and Self-Assembly into SERS-Active Monolayers. Langmuir 2015, 31, (33), 9205-9213.

(59) Chan, W. C. W.; Nie, S., Quantum Dot Bioconjugates for Ultrasensitive Nonisotopic Detection. Science 1998, 281, 2016-2018.

(60) Charron, G.; Hühn, D.; Perrier, A.; Cordier, L.; Pickett, C. J.; Nann, T.; Parak, W. J., On the Use of $\mathrm{pH}$ Titration to Quantitatively Characterize Colloidal Nanoparticles. Langmuir 2012, 28, (43), 15141-15149.

(61) Caballero-Díaz, E.; Pfeiffer, C.; Kastl, L.; Rivera-Gil, P.; Simonet, B.; Valcárcel, M.; Jiménez-Lamana, J.; Laborda, F.; Parak, W. J., The Toxicity of Silver Nanoparticles Depends on Their Uptake by Cells and Thus on Their Surface Chemistry. Particle and Particle Systems Characterization 2013, 30, (12), 1079-1085.

(62) Wu, M. X.; Liu, H.; Liu, J.; Haley, K. N.; Treadway, J. A.; Larson, J. P.; Ge, N.; Peale, F.; Bruchez, M. P., Immunofluorescent labeling of cancer marker Her2 and other cellular targets with semiconductor quantum dots. Nature Biotechnology 2003, 21, 41-46.

(63) Pellegrino, T.; Manna, L.; Kudera, S.; Liedl, T.; Koktysh, D.; Rogach, A. L.; Keller, S.; Rädler, J.; Natile, G.; Parak, W. J., Hydrophobic Nanocrystals Coated with an Amphiphilic Polymer Shell: A General Route to Water Soluble Nanocrystals. Nano Letters 2004, 4, (4), 703-707.

(64) Ali, Z.; Abbasi, A. Z.; Zhang, F.; Arosio, P.; Lascialfari, A.; Casula, M. F.; Wenk, A.; Kreyling, W.; Plapper, R.; Seidel, M.; Niessner, R.; Knoll, J.; Seubert, A.; Parak, W. J., Multifunctional Nanoparticles for Dual Imaging. Analytical Chemistry 2011, 83, (8), 28772882.

(65) Zhang, F.; Lees, E.; Amin, F.; Rivera_Gil, P.; Yang, F.; Mulvaney, P.; Parak, W. J., PolymerCoated Nanoparticles: A Universal Tool for Biolabelling Experiments. Small 2011, 7, 3113-3127.

(66) Pelaz, B.; Del Pino, P.; Maffre, P.; Hartmann, R.; Gallego, M.; Rivera-Fernandez, S.; de la Fuente, J. M.; Nienhaus, G. U.; Parak, W. J., Surface Functionalization of Nanoparticles with Polyethylene Glycol: Effects on Protein Adsorption and Cellular Uptake. ACS Nano 2015, 9, (7), 6996-7008.

(67) Kaiser, U.; Aberasturi, D. J. d.; Vazquez-Gonzalez, M.; Carrillo-Carrion, C.; Niebling, T.; Parak, W. J.; Heimbrodt, W., Determining the exact number of dye molecules attached to colloidal CdSe/ZnS quantum dots in Förster resonant energy transfer assemblies. JOURNAL OF APPLIED PHYSICS 2015, 117, 024701.

(68) Riedinger, A.; Zhang, F.; Dommershausen, F.; Röcker, C.; Brandholt, S.; Nienhaus, G. U.; Koert, U.; Parak, W. J., Ratiometric Optical Sensing of Chloride Ions with Organic 
Fluorophore - Gold Nanoparticle Hybrids: A Systematic Study of Distance Dependency and the Influence of Surface Charge. Small 2010, 6, (22), 2590-2597.

(69) Abbasi, A. Z.; Amin, F.; Niebling, T.; Friede, S.; Ochs, M.; Carregal-Romero, S.; Martos, J. M. M.; Rivera_Gil, P.; Heimbrodt, W.; Parak, W. J., How colloidal nanoparticles could facilitate multiplexed measurements of different analytes with analyte-sensitive organic fluorophores. ACS Nano 2011, 5, 21-25.

(70) Amin, F.; Yushchenko, D. A.; Montenegro, J. M.; Parak, W. J., Integration of Organic Fluorophores in the Surface of Polymer-Coated Colloidal Nanoparticles for Sensing the Local Polarity of the Environment. ChemPhysChem 2012, 13, (4), 1030-1035.

(71) Sperling, R. A.; Liedl, T.; Duhr, S.; Kudera, S.; Zanella, M.; Lin, C.-A. J.; Chang, W. H.; Braun, D.; Parak, W. J., Size Determination of (Bio-) Conjugated Water-Soluble Colloidal Nanoparticles: A Comparison of Different Techniques. J. Phys. Chem. C 2007, 111, (31), 11552-11559.

(72) Harakeh, S.; Abdel-Massih, R. M.; Rivera_Gil, P.; Sperling, R. A.; Meinhardt, A.; Niedwiecki, A.; Rath, M.; Parak, W. J.; Baydoun, E., The effect of PEG-coated gold nanoparticles on the anti-proliferative potential of Specific Nutrient Synergy. Nanotoxicology 2010, 4, 177-185.

(73) Kantner, K.; Ashraf, S.; Carregal-Romero, S.; Carrillo-Carrion, C.; Collot, M.; del Pino, P.; Heimbrodt, W.; Jimenez de Aberasturi, D.; Kaiser, U.; Kazakova, L. I.; Lelle, M.; Martinez de Baroja, N.; Montenegro, J.-M.; Nazarenus, M.; Pelaz, B.; Peneva, K.; Rivera Gil, P.; Sabir, N.; Schneider, L. M.; Shabarchina, L. I.; Sukhorukov, G. B.; Vazquez, M.; Yang, F.; Parak, W. J., Particle-Based Optical Sensing of Intracellular lons at the Example of Calcium - What are the Experimental Pitfalls? Small 2015, 11, 896-904.

(74) Yu, X.; Lei, D. Y.; Amin, F.; Hartmann, R.; Acuna, G. P.; Guerrero-Martínez, A.; Maier, S. A.; Tinnefeld, P.; Carregal-Romero, S.; Parak, W. J., Distance control in-between plasmonic nanoparticles via biological and polymeric spacers. Nano Today 2013, 8, (5), 480-493.

(75) Kaiser, U.; Aberasturi, D. J. d.; Malinowski, R.; Amin, F.; Parak, W. J.; Heimbrodt, W., Multiplexed measurements by time resolved spectroscopy using colloidal CdSe/ZnS quantum dots. Applied Physics Letters 2014, 104, 41901-41904.

(76) Yakovlev, A. V.; Zhang, F.; Zulqurnain, A.; Azhar-Zahoor, A.; Luccardini, C.; Gaillard, S.; Mallet, J. M.; Tauc, P.; Brochon, J. C.; Parak, W. J.; Feltz, A.; Oheim, M., Wrapping Nanocrystals with an Amphiphilic Polymer Preloaded with Fixed Amounts of Fluorophore Generates FRET-Based Nanoprobes with a Controlled Donor/Acceptor Ratio. Langmuir 2009, 25, (5), 3232-3239.

(77) Liedl, T.; Keller, S.; Simmel, F. C.; Rädler, J. O.; Parak, W. J., Fluorescent Nanocrystals as Colloidal Probes in Complex Fluids Measured by Fluorescence Correlation Spectroscopy. Small 2005, 1, (10), 997-1003.

(78) Niebling, T.; Zhang, F.; Ali, Z.; Parak, W. J.; Heimbrodt, W., Excitation dynamics in polymer-coated semiconductor quantum dots with integrated dye molecules: The role of reabsorption. Journal of Applied Physics 2009, 106, 104701.

(79) Fernández-Argüelles, M. T.; Yakovlev, A.; Sperling, R. A.; Luccardini, C.; Gaillard, S.; Medel, A. S.; Mallet, J.-M.; Brochon, J.-C.; Feltz, A.; Oheim, M.; Parak, W. J., Synthesis and Characterization of Polymer-Coated Quantum Dots with Integrated Acceptor Dyes as FRET-based Nanoprobes. Nano Letters 2007, 7, (9), 2613-2617.

(80) Colombo, M.; Mazzucchelli, S.; Montenegro, J. M.; Galbiati, E.; Corsi, F.; Parak, W. J.; Prosperi, D., Protein Oriented Ligation on Nanoparticles Exploiting O6-Alkylguanine-DNA Transferase (SNAP) Genetically Encoded Fusion. Small 2012, 8, (10), 1492-1497. 
(81) Röcker, C.; Pötzl, M.; Zhang, F.; Parak, W. J.; Nienhaus, G. U., A Quantitative Fluorescence Study of Protein Monolayer Formation on Colloidal Nanoparticles. Nat. Nanotechnol. 2009, 4, (9), 577-580.

(82) Lehmann, A. D.; Parak, W. J.; Zhang, F.; Ali, Z.; Röcker, C.; Nienhaus, G. U.; Gehr, P.; Rothen-Rutishauser, B., Fluorescent-Magnetic Hybrid Nanoparticles Induce a DoseDependent Increase in Proinflammatory Response in Lung Cells in vitro Correlated with Intracellular Localization. Small 2010, 6, (6), 753-762.

(83) Mahmoudi, M.; Abdelmonem, A. M.; Behzadi, S.; Clement, J. H.; Dutz, S.; Ejtehadi, M. R.; Hartmann, R.; Kantner, K.; Linne, U.; Maffre, P.; Metzler, S.; Moghadam, M. K.; Pfeiffer, C.; Rezaei, M.; Ruiz-Lozano, P.; Serpooshan, V.; Shokrgozar, M. A.; Nienhaus, G. U.; Parak, W. J., Temperature: The "Ignored" Factor at the NanoBio Interface. ACS Nano 2013, 7, (8), 6555-6562.

(84) Tromsdorf, U. I.; Bigall, N. C.; Kaul, M.; Bruns, O. T.; Nikolic, M. S.; Mollwitz, B.; Sperling, R. A.; Reimer, R.; Hohenberg, H.; Parak, W. J.; Förster, S.; Beisiegel, U.; Adam, G.; Weller, $\mathrm{H}$., Size and Surface Effects on the MRI Relaxivity of Manganese Ferrite Nanoparticle Contrast Agents. Nano Letters 2007, 7, (8), 2422-2427.

(85) Abdelmonem, A. M.; Pelaz, B.; Kantner, K.; Bigall, N. C.; Del Pino, P.; Parak, W. J., Charge and agglomeration dependent in vitro uptake and cytotoxicity of zinc oxide nanoparticles. Journal of Inorganic Biochemistry 2015, 153, 334-338.

(86) Kim, G.-M.; Wutzler, A.; Radusch, H.-J.; Michler, G. H.; Simon, P.; Sperling, R. A.; Parak, W. J., One-Dimensional Arrangement of Gold Nanoparticles by Electrospinning. Chemistry of Materials 2005, 17, 4949-4957.

(87) Geidel, C.; Schmachtel, S.; Riedinger, A.; Pfeiffer, C.; Müllen, K.; Klapper, M.; Parak, W. J., A General Synthetic Approach for Obtaining Cationic and Anionic Inorganic Nanoparticles via Encapsulation in Amphiphilic Copolymers. Small 2011, 7, (20), 29292934.

(88) Hühn, D.; Kantner, K.; Geidel, C.; Brandholt, S.; De Cock, I.; Soenen, S. J. H.; Rivera Gil, P.; Montenegro, J.-M.; Braeckmans, K.; Müllen, K.; Nienhaus, G. U.; Klapper, M.; Parak, W. J., Polymer-Coated Nanoparticles Interacting with Proteins and Cells: Focusing on the Sign of the Net Charge. ACS Nano 2013, 7, (4), 3253-3263.

(89) Hagel, L., Gel Filtration. In Protein Purification. Principles, High Resolution Methods and Applications, 2 ed.; Janson, J.-C.; Ryden, L., 'Ed.'^'Eds.' John Wiley \& Sons: New York, 1998; 'Vol.' p^pp 79-143.

(90) Amersham_Pharmacia_Biotech, Gel filtration - Principles and Methods. 8 ed.; Amersham Pharmacia Biotech: 2001; 'Vol.' p.

(91) Parak, W. J.; Gerion, D.; Zanchet, D.; Woerz, A. S.; Pellegrino, T.; Micheel, C.; Williams, S. C.; Seitz, M.; Bruehl, R. E.; Bryant, Z.; Bustamante, C.; Bertozzi, C. R.; Alivisatos, A. P., Conjugation of DNA to silanized colloidal semiconductor nanocrystaline quantum dots. Chemistry of Materials 2002, 14, (5), 2113-2119.

(92) Zanchet, D.; Micheel, C. M.; Parak, W. J.; Gerion, D.; Williams, S. C.; Alivisatos, A. P., Electrophoretic and Structural Studies of DNA-Directed Au Nanoparticle Groupings. Journal of Physical Chemistry B 2002, 106, (45), 11758-11763.

(93) Robin Harris, J., Negative Staining of Thinly Spread Biological Particulates. In Electron Microscopy Methods and Protocols, ed.; 'Ed.'^'Eds.' Humana Press: 1999; 'Vol.' 117, $p^{\wedge} p p$.

(94) Carpenter, A.; Jones, T.; Lamprecht, M.; Clarke, C.; Kang, I.; Friman, O.; Guertin, D.; Chang, J.; Lindquist, R.; Moffat, J.; Golland, P.; Sabatini, D., CellProfiler: image analysis software for identifying and quantifying cell phenotypes. Genome Biology 2006, 7, (10), R100. 
(95) Borchert, H.; Shevchenko, E. V.; Robert, A.; Mekis, I.; Kornowski, A.; Grübel, G.; Weller, H., Determination of Nanocrystal Sizes: A Comparison of TEM, SAXS, and XRD Studies of Highly Monodisperse CoPt 3 Particles. Langmuir 2005, 21, (5), 1931-1936.

(96) Dubois, L. H.; Nuzzo, R. G., Synthesis, Structure, and Properties of Model OrganicSurfaces. Annual Review of Physical Chemistry 1992, 43, 437-463.

(97) Fedosyuk, A.; Radchanka, A.; Antanovich, A.; Prudnikau, A.; Kvach, M. V.; Shmanai, V.; Artemyev, M., Determination of Concentration of Amphiphilic Polymer Molecules on the Surface of Encapsulated Semiconductor Nanocrystals. Langmuir 2016, 32, (8), 19551961.

(98) Liz-Marzan, L. M., Tailoring surface plasmons through the morphology and assembly of metal nanoparticles. Langmuir 2006, 22, (1), 32-41.

(99) Sepulveda, B.; Angelome, P. C.; Lechuga, L. M.; Liz-Marzan, L. M., LSPR-based nanobiosensors. Nano Today 2009, 4, (3), 244-251.

(100) Barbosa, S.; Agrawal, A.; Rodriguez-Lorenzo, L.; Pastoriza-Santos, I.; Alvarez-Puebla, R. A.; Kornowski, A.; Weller, H.; Liz-Marzan, L. M., Tuning Size and Sensing Properties in Colloidal Gold Nanostars. Langmuir 2010, 26, (18), 14943-14950.

(101) Vial, S.; Pastoriza-Santos, I.; Perez-Juste, J.; Liz-Marzan, L. M., Plasmon coupling in layerby-layer assembled gold nanorod films. Langmuir 2007, 23, (8), 4606-4611.

(102) Efros, A. L.; L.Efros, A., INTERBAND ABSORPTION OF LIGHT IN A SEMICONDUCTOR SPHERE. SOVIET PHYSICS SEMICONDUCTORS-USSR 1982, 16, (7), 772-775.

(103) Takagahara, T.; Takeda, K., Theory of the quantum confinement effect on excitons in quantum dots of indirect-gap materials. Physical Review B 1992, 46, 15578-15581.

(104) Vossmeyer, T.; Katsikas, L.; Giersig, M.; Popovic, I. G.; Diesner, K.; Chemseddine, A.; Eychmüller, A.; Weller, H., CdS Nanoclusters: Synthesis, Characterization, Size Dependent Oscillator Strength, Temperature Shift of the Excitonic Transition Energy, and Reversible Absorbance Shift. J. Phys. Chem. 1994, 98, 7665 -.

(105) Nirmal, M.; Murray, C. B.; Bawendi, M. G., Fluorescence-line narrowing in CdSe quantum dots: Surface localization of the photogenerated exciton. Physical Review B 1994, 50, (4), 2293-2300.

(106) Lavallard, P., Excitons in nanocrystals. Journal of Crystal Growth 1998, 185, 352-359.

(107) Peng, X. G.; Manna, L.; Yang, W. D.; Wickham, J.; Scher, E.; Kadavanich, A.; Alivisatos, A. P., Shape control of CdSe nanocrystals. Nature 2000, 404, (6773), 59-61.

(108) Henglein, A., Photo-Degradation And Fluorescence Of Colloidal-Cadmium Sulfide In Aqueous-Solution. Berichte Der Bunsen-Gesellschaft-Physical Chemistry Chemical Physics 1982, 86, (4), 301-305.

(109) Rossetti, R.; Nakahara, S.; Brus, L. E., Quantum Size Effects in the Redox Potentials, Resonance Raman-Spectra, and Electronic-Spectra of Cds Crystallites in AqueousSolution. Journal of Chemical Physics 1983, 79, (2), 1086-1088.

(110) Ekimov, A. I.; Efros, A. L.; Onushchenko, A. A., Quantum size effect in semiconductor microcrystals. Solid State Communications 1993, 88, 947-50.

(111) Alivisatos, A. P., Semiconductor Clusters, Nanocrystals, and Quantum Dots. Science 1996, 271, (16 February), 933-937.

(112) Parak, W. J.; Manna, L.; Simmel, F. C.; Gerion, D.; Alivisatos, P., Quantum Dots. In Nanoparticles - From Theory to Application, 1 ed.; Schmid, G., 'Ed.'^'Eds.' Wiley-VCH: Weinheim, 2004; 'Vol.' p^pp 4-49.

(113) Segets, D.; Lucas, J. M.; Taylor, R. N. K.; Scheele, M.; Zheng, H.; Alivisatos, A. P.; Peukert, W., Determination of the Quantum Dot Band Gap Dependence on Particle Size from Optical Absorbance and Transmission Electron Microscopy Measurements. ACS Nano 2012, 6, (10), 9021-9032. 
(114) Haiss, W.; Thanh, N. T. K.; Aveyard, J.; Fernig, D. G., Determination of Size and Concentration of Gold Nanoparticles from UV-Vis Spectra. Anal. Chem. 2007, 79, (11), 4215-4221.

(115) Striolo, A.; Ward, J.; Prausnitz, J. M.; Parak, W. J.; Zanchet, D.; Gerion, D.; Milliron, D. J.; Alivisatos, A. P., Molecular Weight, Osmotic Second Virial Coefficient, and Extinction Coefficient of colloidal CdSe Nanocrystals. Journal of Physical Chemistry B 2002, 106, (21), 5500-5505.

(116) Menendez-Miranda, M.; Costa-Fernandez, J. M.; Encinar, J. R.; Parak, W. J.; CarrilloCarrion, C., Determination of the ratio of fluorophore/nanoparticle for fluorescencelabelled nanoparticles. Analyst 2016, 141, (4), 1266-1272.

(117) Bruchez, M. J.; Moronne, M.; Gin, P.; Weiss, S.; Alivisatos, A. P., Semiconductor Nanocrystals as Fluorescent Biological Labels. Science 1998, 281, (5385), 2013-2016.

(118) Wilhelm, S.; Kaiser, M.; Wuerth, C.; Heiland, J.; Carrillo-Carrion, C.; Muhr, V.; Wolfbeis, O. S.; Parak, W. J.; Resch-Genger, U.; Hirsch, T., Water dispersible upconverting nanoparticles: effects of surface modification on their luminescence and colloidal stability. Nanoscale 2015, 7, (4), 1403-1410.

(119) Lin, C.-A. J.; Yang, T.-Y.; Lee, C.-H.; Huang, S. H.; Sperling, R. A.; Zanella, M.; Li, J. K.; Shen, J.-L.; Wang, H.-H.; Yeh, H.-I.; Parak, W. J.; Chang, W. H., Synthesis, Characterization, and Bioconjugation of Fluorescent Gold Nanoclusters toward Biological Labeling Applications. ACS Nano 2009, 3, (2), 395-401.

(120) Huang, S.; Pfeiffer, C.; Hollmann, J.; Friede, S.; Chen, J. J.-C.; Beyer, A.; Volz, K.; Heimbrodt, W.; Montenegro Martos, J. M.; Chang, W.; Parak, W. J., Synthesis and Characterization of Colloidal Fluorescent Silver Nanoclusters. Langmuir 2012, 28, 8915-8919.

(121) Dulkeith, E.; Ringler, M.; Klar, T. A.; Feldmann, J.; Muñoz Javier, A.; Parak, W. J., Gold Nanoparticles Quench Fluorescence by Phase Induced Radiative Rate Suppression. Nanoletters 2005, 5, (4), 585-589.

(122) Medintz, I. L.; Clapp, A. R.; Mattoussi, H.; Goldman, E. R.; Fisher, B.; Mauro, J. M., SelfAssembled Nanoscale Biosensors Based on Quantum Dot FRET Donors. Nat. Mater. 2003, 2, 630-638.

(123) Würth, C.; Geissler, D.; Behnke, T.; Kaiser, M.; Resch-Genger, U., Critical review of the determination of photoluminescence quantum yields of luminescent reporters. Analytical and Bioanalytical Chemistry 2015, 407, 59-78.

(124) Huber, A.; Behnke, T.; Würth, C.; Jaeger, C.; Resch-Genger, U., Spectroscopic Characterization of Coumarin-Stained Beads: Quantification of the Number of Fluorophores Per Particle with Solid-State 19F-NMR and Measurement of Absolute Fluorescence Quantum Yields. Analytical Chemistry 2012, 84, (8), 3654.

(125) Jobin_Yvon_Horiba, A Guide to Recording Fluorescence Quantum Yields. In ed.; 'Ed. '^`Eds.' Jobin Yvon - Horiba: Stanmore, 2016; 'Vol.' p^pp.

(126) Magde, D.; Brannon, J. H.; Cremers, T. L.; Olmsted, J., Absolute Luminescence Yield of Cresyl Violet - Standard for the Red. J. Phys. Chem. 1979, 83, (6), 696-699.

(127) Rivera Gil, P.; Jimenez de Aberasturi, D.; Wulf, V.; Pelaz, B.; del Pino, P.; Zhao, Y.; de la Fuente, J.; Ruiz de Larramendi, I.; Rojo, T.; Liang, X.-J.; Parak, W. J., The Challenge to Relate the Physicochemical Properties of Colloidal Nanoparticles to Their Cytotoxicity. Accounts of Chemical Research 2013, 46, (3), 743-749.

(128) Murdock, R. C.; Braydich-Stolle, L.; Schrand, A. M.; Schlager, J. J.; Hussain, S. M., Characterization of nanomaterial dispersion in solution prior to in vitro exposure using dynamic light scattering technique. Toxicol Sci 2008, 101, (2), 239-53.

(129) Roebben, G.; Ramirez-Garcia, S.; Hackley, V. A.; Roesslein, M.; Klaessig, F.; Kestens, V.; Lynch, I.; Garner, C. M.; Rawle, A.; Elder, A.; Colvin, V. L.; Kreyling, W.; Krug, H. F.; 
Lewicka, Z. A.; McNeil, S.; Nel, A.; Patri, A.; Wick, P.; Wiesner, M.; Xia, T.; Oberdorster, G.; Dawson, K. A., Interlaboratory comparison of size and surface charge measurements on nanoparticles prior to biological impact assessment. Journal of Nanoparticle Research 2011, 13, (7), 2675-2687.

(130) Bhattacharjee, S., DLS and zeta potential - What they are and what they are not? Journal of Controlled Release 2016.

(131) Tay, C. Y.; Setyawati, M. I.; Xie, J.; W. J. Parak; Leong, D. T., Back to Basics: Exploiting the Innate Physico-chemical Characteristics of Nanomaterials for Biomedical Applications. Advanced Functional Materials 2014, 24, 5936 - 5955.

(132) Henderson, L. J., Concerning the relationship between the strength of acids and their capacity to preserve neutrality. American Journal of Physiology 1908, 21, (2), 173-179.

(133) Hasselbalch, K. A., Die Berechnung der Wasserstoffzahl des Blutes aus der freien und gebundenen Kohlensäure desselben, und die Sauerstoffbindung des Blutes als Funktion der Wasserstoffzahl. Biochemische Zeitschrift 1917, 78, 112-144.

(134) Po, H. N.; Senozan, N. M., The Henderson-Hasselbalch Equation: Its History and Limitations. Journal of Chemical Education 2001, 78, (11), 1499.

(135) de Levie, R., The Henderson Approximation and the Mass Action Law of Guldberg and Waage. The Chemical Educator 2002, 7, (3), 132-135.

(136) de Levie, R., The Henderson-Hasselbalch Equation: Its History and Limitations. Journal of Chemical Education 2003, 80, (2), 146.

(137) Doane, T. L.; Chuang, C. H.; Hill, R. J.; Burda, C., Nanoparticle Zeta-Potentials. Accounts of Chemical Research 2012, 45, (3), 317-326. 


\section{Appendix: Used symbols}

$\mathrm{d}_{\mathrm{c}}$ : diameter of inorganic core

$r_{c}=d_{c} / 2:$ radius of inorganic core

$d_{c 1}, d_{c 2}, \ldots$ : diameter NP after growth of different inorganic shells

$l_{\text {ligand: }}$ thickness of the organic ligand shell

$d_{\text {eff: }}$ effective NP diameter $\left(d_{c}+2 \cdot l_{\text {ligand }}\right)$

$\mathrm{d}_{\mathrm{h}}$ : hydrodynamic diameter of NP in solvent

$\mathrm{L}_{c}$ : length if inorganic core in case of rod-shaped NPS

$L_{\text {eff }}$ : effective NP length $\left(L_{c}+2 I_{\text {ligand }}\right)$

$A_{c}$ : surface of one single NP core

$A_{\text {eff: }}$ effective surface area of one single NP

$A_{\text {total_eff }}$ : total effective surface area of all the NPs in a solution

$\mathrm{V}_{\mathrm{c}}$ : volume of one NP core

$\mathrm{V}_{\mathrm{c} 12}$ : volume of the inorganic core (1) and shell (2) of one single NP

$V_{c i}$ : volume of inorganic shell $i=V_{c, i, i-1}-V_{c, i-1}$ of one single

$\mathrm{m}_{\mathrm{NP}}$ : mass of one single NP

$m_{c}$ : mass of one single NP core

$m_{N P, \text { org }}$ : mass of the organic coating of one single NP

$\mathrm{M}_{\mathrm{c}}$ : molar mass of NP cores

$M_{x}, M_{w}(x)$ : molar mass of an element (or material) $x$

$\rho_{\mathrm{x}}$ : bulk densities of a material $\mathrm{x}$

$\mathrm{N}_{\mathrm{P} / \mathrm{NP}}=$ number of polymer monomer units per NP

$\mathrm{N}_{\mathrm{x} / \mathrm{NP}}$ : number of atoms $\mathrm{x}$ per NP

$\mathrm{N}_{\text {dye/NP: }}$ number of dye molecules per NP

$\mathrm{C}_{\mathrm{NP}}$ : molar concentration of NPS

$C_{P}$ : molar polymer monomer concentration

$c_{x}$ : molar concentration of molecule or element $x$

$c_{\text {dye }}$ : molar concentration of dye

$\mathrm{V}_{\mathrm{NP}_{-} \text {sol }}$ : volume of NP suspension

$V_{P_{-} \text {sol }}$ : volume of polymer solution

$\mathrm{V}_{\mathrm{x}_{-} \text {sol }}$ : volume of solution of compound $\mathrm{x}$

$\mathrm{V}_{\mathrm{NP} \_ \text {pel }}$ : volume of NP pellet

$V_{P_{\text {_pel }}}$ : volume of polymer pellet

$V_{x_{-} \text {pel }}$ : volume of pellet of compound $x$

$\mathrm{m}_{\mathrm{NP} \_ \text {pel }}$ : mass of NP pellet

$\mathrm{N}_{\mathrm{NP}}=$ number of NPs

$\mathrm{n}_{\mathrm{NP}}=$ amount of NPs

$n_{P}=$ amount of polymer monomer units 
$\mathrm{R}_{\text {chain } / \text { mon }}=$ percentage of monomer units that are modified with a hydrophobic side chain

$\mathrm{R}_{\text {cross } / \text { mon }}=$ percentage of monomer units that are modified with a crosslinker molecule $\mathrm{R}_{\text {func/mon }}=$ percentage of monomer units that are modified with a functional molecules (such as an organic fluorophore)

$\mathrm{N}_{\mathrm{A}}=$ Avogadro's number

RT: room temperature

$\mathrm{T}$ : temperature 